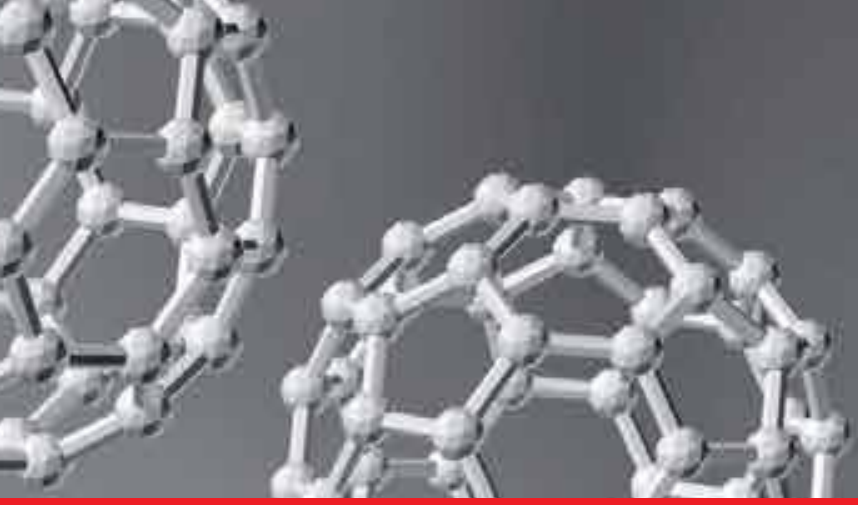

\title{
IntechOpen
}

\section{Role of Novel Drug Delivery Vehicles in Nanobiomedicine}

Edited by Rajeev K. Tyagi, Neeraj Garg, Rahul Shukla and Prakash Singh Bisen
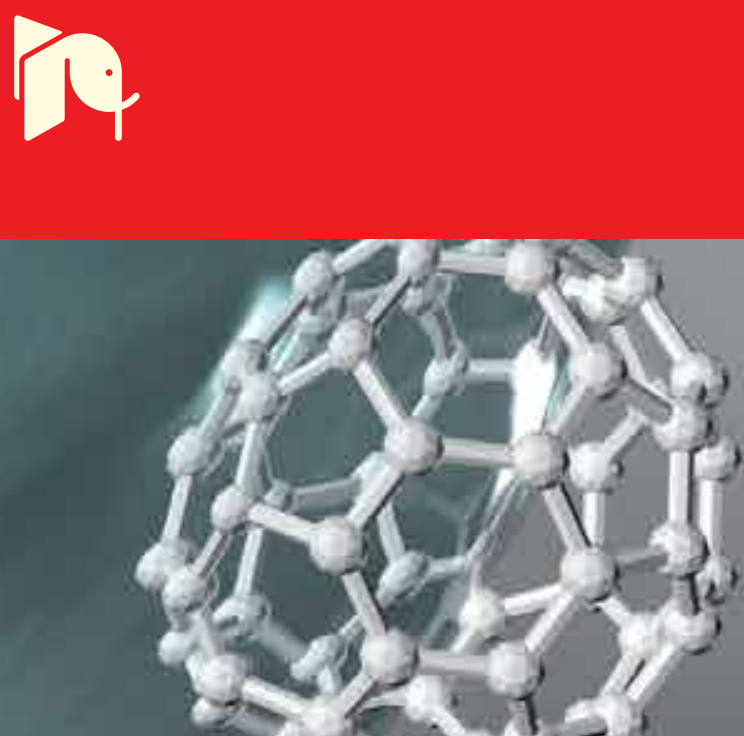



\section{Role of Novel Drug Delivery Vehicles in Nanobiomedicine}

Edited by Rajeev K. Tyagi, Neeraj Garg, Rahul Shukla and Prakash Singh Bisen 

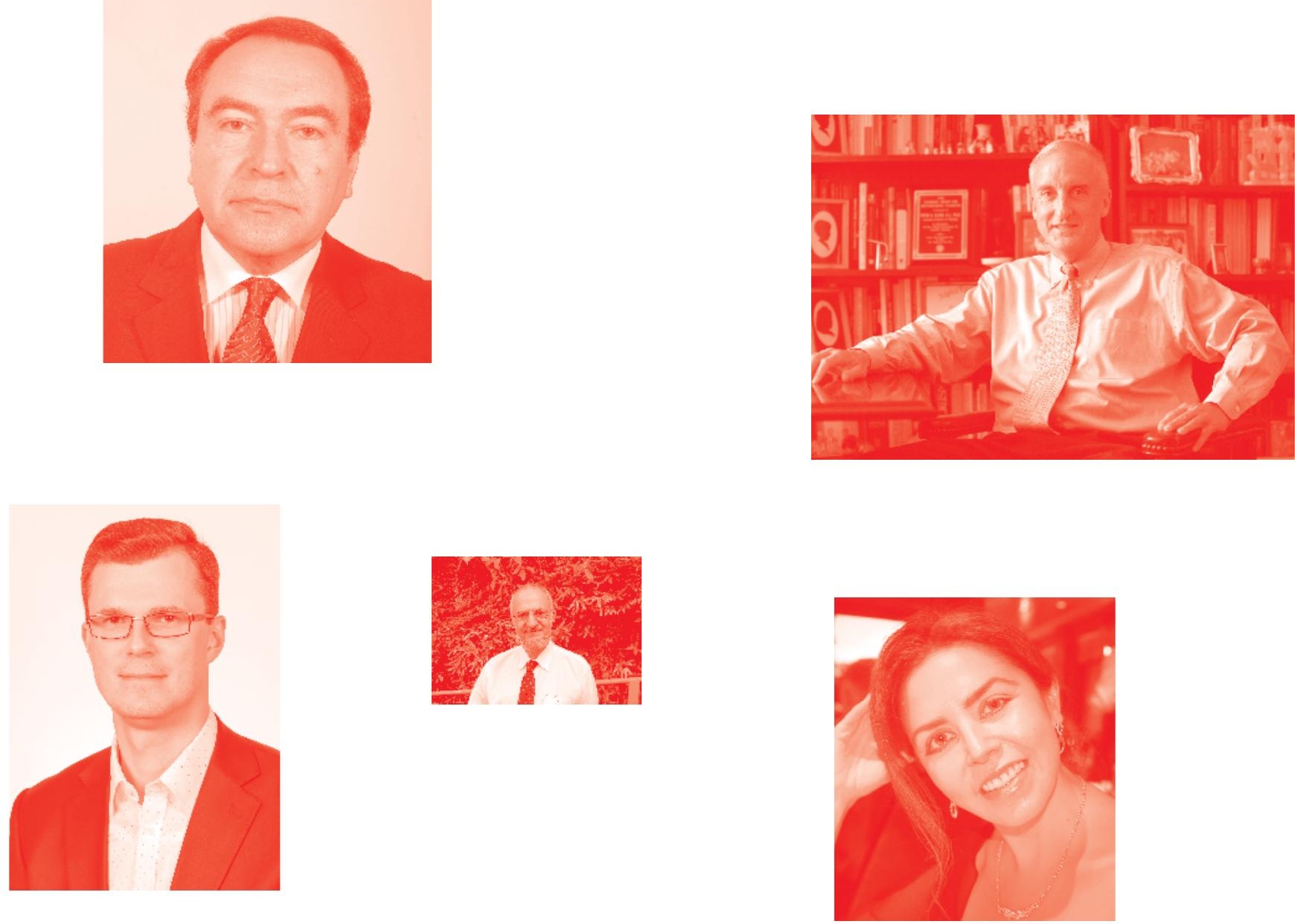

Supporting open minds since 2005
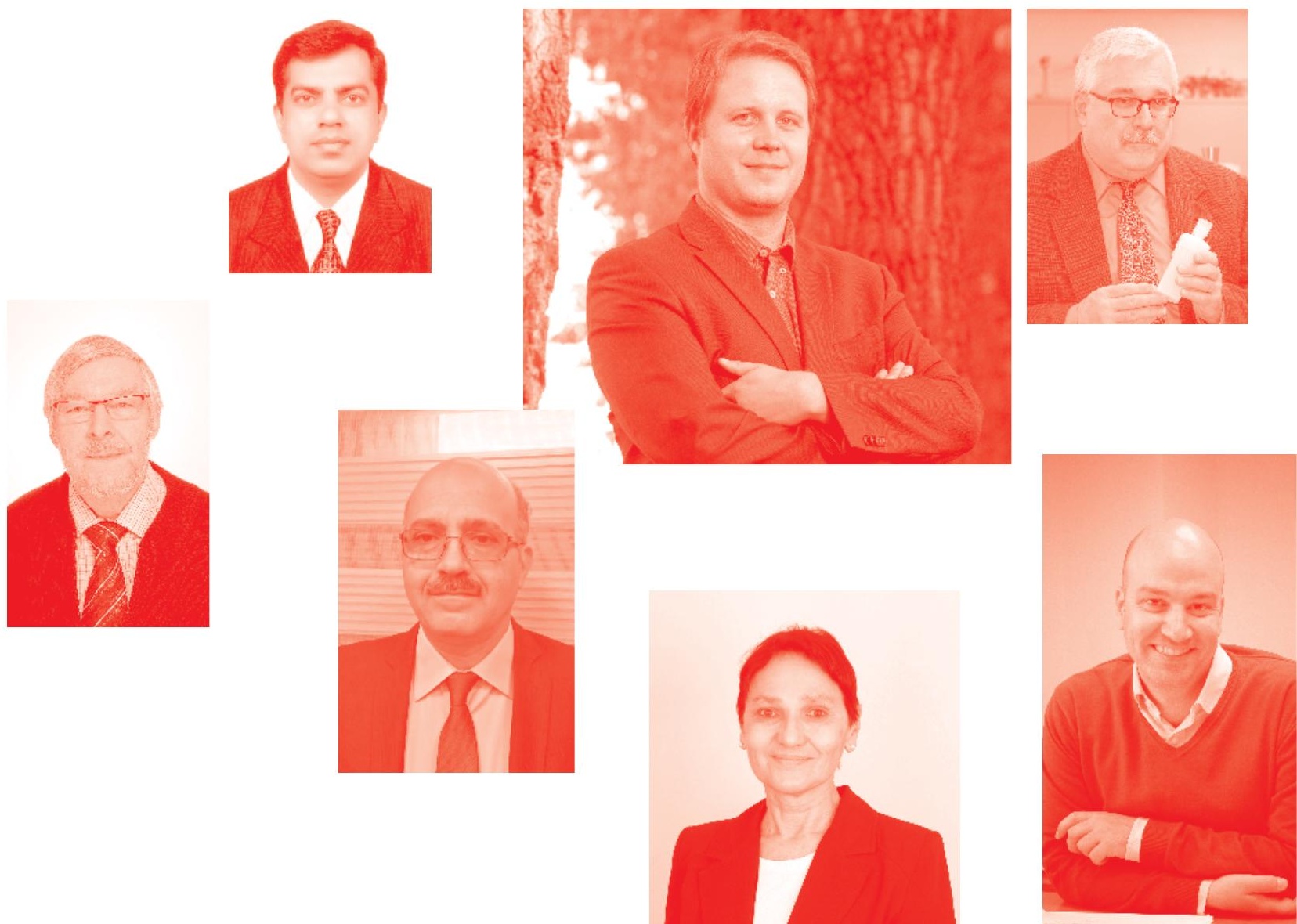
Role of Novel Drug Delivery Vehicles in Nanobiomedicine

http: //dx. doi.org/10.5772/intechopen. 77468

Edited by Rajeev K. Tyagi, Neeraj Garg, Rahul Shukla and Prakash Singh Bisen

\section{Contributors}

Muniasamy Neerathilingam, Chetan Chandola, Arturo Santos, Alejandro Gonzalez-De La Rosa, Jose Navarro-Partida, Juan Carlos Altamirano-Vallejo, Jane Hsiao, Nayab Tahir, Muhammad Tahir Haseeb, Asadullah Madni, Farzana Parveen, Muhammad Muzamil Khan, Safiullah Khan, Nasrullah Jan, Arshad Khan, Jinu George, Reshma Joy, Franklin John, Subas Chandra Dinda, Bibhash Mohanta, Narahari Narayan Palei, Jyotirmoy Deb, Aadesh Kumar, Mahendra Rana, Amita Rana, Chander Amgoth, Chiuyen Phan, Murali Banavoth, Santosh Rompivalasa, Guping Tang, Weifen Zhang, Xiuwen Guan

( ) The Editor(s) and the Author(s) 2020

The rights of the editor(s) and the author(s) have been asserted in accordance with the Copyright, Designs and Patents Act 1988. All rights to the book as a whole are reserved by INTECHOPEN LIMITED. The book as a whole (compilation) cannot be reproduced, distributed or used for commercial or non-commercial purposes without INTECHOPEN LIMITED's written permission. Enquiries concerning the use of the book should be directed to INTECHOPEN LIMITED rights and permissions department (permissions@intechopen.com).

Violations are liable to prosecution under the governing Copyright Law .

\section{(cc) BY}

Individual chapters of this publication are distributed under the terms of the Creative Commons Attribution 3. 0 Unported License which permits commercial use, distribution and reproduction of the individual chapters, provided the original author(s) and source publication are appropriately acknowledged. If so indicated, certain images may not be included under the Creative Commons license. In such cases users will need to obtain permission from the license holder to reproduce the material. More details and guidelines concerning content reuse and adaptation can be found at http : //www . intechopen . com/copyright-policy . html .

\section{Notice}

Statements and opinions expressed in the chapters are these of the individual contributors and not necessarily those of the editors or publisher. No responsibility is accepted for the accuracy of information contained in the published chapters. The publisher assumes no responsibility for any damage or injury to persons or property arising out of the use of any materials, instructions, methods or ideas contained in the book.

First published in London, United Kingdom, 2020 by IntechOpen IntechOpen is the global imprint of INTECHOPEN LIMITED, registered in England and Wales, registration number: 11086078 , 7th floor, 10 Lower Thames Street, London,

EC3R 6AF, United Kingdom

Printed in Croatia

British Library Cataloguing-in-Publication Data

A catalogue record for this book is available from the British Library

Additional hard and PDF copies can be obtained from orders@intechopen.com

Role of Novel Drug Delivery Vehicles in Nanobiomedicine

Edited by Rajeev K. Tyagi, Neeraj Garg, Rahul Shukla and Prakash Singh Bisen

p. cm.

Print ISBN 978-1-78923-985- 0

Online ISBN 978-1-78923-986-7

eBook (PDF) ISBN 978-1-78984-330-9 


\section{We are IntechOpen, \\ the world's leading publisher of Open Access books}

\section{Built by scientists, for scientists}

\section{$4,600+$}

Open access books available

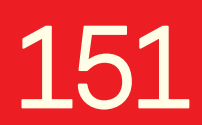

Countries delivered to

\section{$120,000+$}

International authors and editors

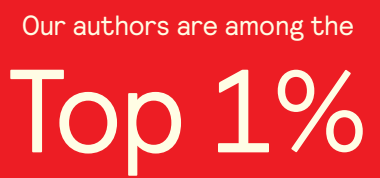

most cited scientists

Contributors from top 500 universities
$135 \mathrm{M}+$

Downloads
$12.2 \%$

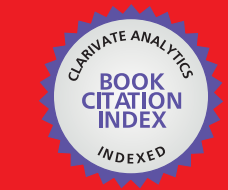

WEB OF SCIENCE ${ }^{\text {M }}$

Selection of our books indexed in the Book Citation Index in Web of Science ${ }^{\mathrm{TM}}$ Core Collection (BKCI)

\section{Interested in publishing with us? \\ Contact book.department@intechopen.com}

Numbers displayed above are based on latest data collected.

For more information visit www.intechopen.com 



\section{Meet the editors}

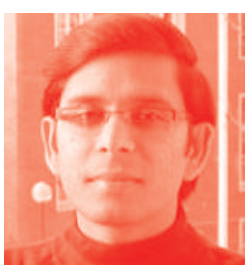

Dr. Rajeev K. Tyagi completed his Master's degree in Biotechnology at Meerut University, India. He completed his doctorate (PhD) in Immunology/Parasitology/Infectiology with a special mention of "Tres Honorable" at the Biomedical Parasitology Unit, Institute Pasteur, Paris, France (2011) and developed a preclinical "humanized" mouse model(s) to study asexual blood and liver-stage infection of Plasmodium falciparum. Dr. Tyagi worked at the University of South Florida, Augusta University, and Vanderbilt University Medical Center, USA, as a postdoctoral scientist and deployed human chimeric mice to study $P$. falciparum and understand the immunological pathways of colitis. He has been working to develop novel drug delivery systems to deliver methotrexate and aceclofenac to explore their antiinflammatory effect in breast cancer, rheumatoid arthritis, etc. Dr. Tyagi has been employing his efforts to combine translational biomedical research with nanotechnology to develop therapeutic interventional approaches for inflammatory diseases and beyond. Currently, he has been working as a scientist at the CSIR-Institute of Microbial Technology (IMTECH), Chandigarh, India.

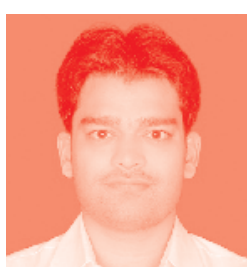

Dr. Neeraj K. Garg is presently working as a formulation scientist (Manager) in Sun Pharmaceutical Industries Limited, Vadodara, India. His doctoral research focused on-to develop and optimize the novel lipid and polymer based nanocarriers for delivery of immunosuppressive drugs. Dr Neeraj have been working in the field of Advanced Drug Delivery System for last 15 years. He is working in the area of targeted lipid and polymeric nanoparticles in general and development of Lipid and polymer based drug delivery vehicles; polymeric nanoparticles, solid lipid nanoparticles, lipid-polymer hybrid nanoparticles, nanostructure lipid carriers (NLCs), in particular.

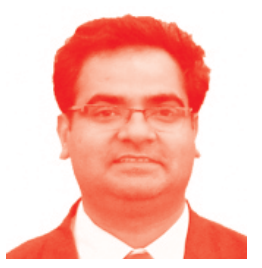

Dr. Rahul Shukla is presently working as an assistant professor in the Department of Pharmaceutics, National Institute of Pharmaceutical Education and Research (Raebareli), India. He was awarded a PhD from JNU, New Delhi, a CSIR-CDRI and MPharm in Pharmaceutics from IIT-BHU, Varanasi, and a D.S. Kothari Post Doctoral Fellowship from Panjab University, Chandigarh. He had experience as a formulation scientist at Dr. Reddy's laboratory, Hyderabad. He is presently guiding both doctoral students and master's students. He has published widely in areas of nanomedicine and targeted drug delivery systems, particle engineering, controlled release systems, neglected and tropical disease therapeutics, product development, and nutraceuticals in internationally renowned journals and books. He has delivered various lectures on many national and international platforms. 


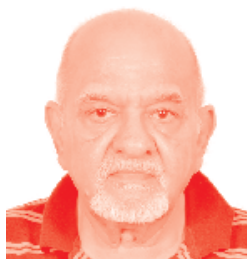

Professor P.S. Bisen, received PhD in 1972 and D.Sc. in 1981 from Jabalpur University, Jabalpur, India, guided 65 doctoral theses having 350 research publications and reviews to his credit in indexed international journals of repute with 12 international books (Springer, John Wiley, CRC Press and IntechOpen) on contemporary issues in Biological Sciences, served as Director of the Institute of Microbiology and Biotechnology, Barkatullah University, Bhopal, Vice-Chancellor, Jiwaji University, Gwalior, Direcor, Madhav Institute of Technology and Science, Gwalior, Director and Dean, JC Bose Institute of Life Sciences, Bundelkhand University, Jhansi and Principal Director and Advisor, Jaipur National University, Jaipur. He was a fellow of the UNESCO/UNDP/ ICRO and Hungarian Academy of Sciences at the Institute of Plant Physiology, Biological Research Center, Szeged, Hungary; U.S. National Science Foundation Fellow in the Department of Biological Sciences at the University of Illinois at Chicago and in the Department of Bacteriology at the University of California, Davis; UNESCO/ WHO fellow at the Institute of Microbiology, Czechoslovak Academy of Sciences, Budejovika, Praha, Czechoslovakia; European Commission's Marie Curie fellow at the Institute of Environmental and Biological Sciences, University of Lancaster, United Kingdom; and DAAD (German Academic Exchange Fellowship) fellow at the University of Bonn, Germany, awardees of First Indira Gandhi Fellowship for his outstanding work on environment conservation and management, National Overseas Associate ship of the Department of Biotechnology, Ministry of Science and Technology, Govt. of India, UGC Career Award, State Level Kailash Nath Katju Award of Government of Madhya Pradesh for contributions in Life Sciences. He was awarded the Life Time Professorship, the highest honor of Bundelkhand University, Jhansi, India. He has also worked as emeritus scientist (CSIR) at Defense Research Development Establishment, Defence Research Development Organization, Ministry of Defense, Government of India, Gwalior and is currently pursuing his research in the field of medical biotechnology as Advisor in the Institute of Medical Sciences, Jaipur National University, Jaipur with a research project on tuberculosis from Grand Challenges Canada, Govt. of Canada. Professor Bisen is the elected fellow of the National Academy of Sciences, India, for his enormous contributions to the field of biology. The diagnostic technology developed by Professor Bisen is unique in several respects and has received recognition worldwide. The technology has been patented in India, the United States, Europe, and Japan. He has served as the external scientific advisor to a Barcelona-based Spanish multinational company, Biokit, involved in the manufacturing of diagnostic kits and also serving as Scientific Adviser of an upcoming food industry, Tropilite Foods, based at Gwalior involved on probiotic research, food biotechnology and develops several innovative products for the food industry. Beijing Doing Institute of Translational Medicine Medical Group, China and Nan Yang Academy of Science Singapore appointed him as Senior Research Advisor. 


\section{Contents}

Chapter 1

Aptamers for Targeted Delivery: Current Challenges and Future Opportunities by Chetan Chandola and Muniasamy Neerathilingam

Chapter 2

Breaking down the Barrier: Topical Liposomes as Nanocarriers for Drug

Delivery into the Posterior Segment of the Eyeball

by Arturo Santos, Juan C. Altamirano-Vallejo, José Navarro-Partida,

Alejandro González-De la Rosa and Jane H. Hsiao

Chapter 3

Lipid Polymer Hybrid Nanoparticles: A Novel Approach for Drug Delivery by Nayab Tahir, Muhammad Tahir Haseeb, Asadullah Madni, Farzana Parveen, Muhammad Muzamil Khan, Safiullah Khan, Nasrullah Jan and Arshad Khan

Chapter 4

Preparation and Characterisation of Niosomal Emulsions as Novel Drug Delivery Vehicle Derived from Natural Seaweeds

by Reshma Joy, Franklin John and Jinu George

Chapter 5

Solid Lipid Based Nano-particulate Formulations in Drug Targeting by Bibhash Chandra Mohanta, Subas Chandra Dinda, Narahari Narayan Palei and Jyotirmoy Deb

Chapter 6

Drug Delivery through Targeted Approach with Special References to Phytosomes by Mahendra Rana, Aadesh Kumar and Amita J. Rana

Chapter 7

Polymer Properties: Functionalization and Surface Modified Nanoparticles by Chander Amgoth, Chiuyen Phan, Murali Banavoth, Santosh Rompivalasa and Guping Tang

Chapter 8

Applications of Chitosan in Pulmonary Drug Delivery

by Xiuwen Guan and Weifen Zhang 



\section{Preface}

As we researched the currently available textbooks covering the basic principles, applications, and promises of nanotechnology as it applies to medicine, we noted a dearth of introductory material tailored specifically for students. While a number of comprehensive books exist outlining the promise of nanosciences applicable to medical applications, including the most recent advances in medical research, these texts fail to properly introduce the student to nanoscience and nanotechnology because they apply first to biology and then to potential therapeutics and diagnostics applications. Thus, this textbook is devoted to the basic principles of nanotechnology, focusing on nanomaterials and nanoparticles, because with respect to the whole of nanoscience, these sectors hold promise for the future of medicine. It is tailored towards real-world applications of medical nanotechnologies with a heavy emphasis on specific examples from the existing literature available in this area. This book is organized to naturally transition from a basic understanding of the principles, including physics, behind, for example, nanoparticles and nanomaterials, to how these principles might be exploited and used to treat or at the very least efficiently diagnose human diseases or anomalies. Each chapter introduces topics and vocabulary at a very basic level and transitions to more advanced coverage as the student's knowledge level matures.

Dr. Rajeev K. Tyagi, PhD

Ramalingaswami Fellow and Faculty, Division of Cell Biology and Immunology, Biomedical Parasitology and Nano-immunology Lab, CSIR-Institute of Microbial Technology (IMTECH), Chandigarh, India

Dr. Rahul Shukla, PhD Assistant Professor, Department of Pharmaceutics, National Institute of Pharmaceutical Education and Research, Raebareli, Lucknow, India

Dr. Neeraj K. Garg Formulation Scientist, Sun Pharmaceutical Industries Limited, Vadodara, India

Prakash S. Bisen Professor and Advisor, Institute of Medical Sciences, Jaipur National University, Jaipur 
Emeritus Professor,

School of Studies in Biotechnology,

Jiwaji University, Gwalior 


\title{
Chapter 1
}

\section{Aptamers for Targeted Delivery: Current Challenges and Future Opportunities}

\author{
Chetan Chandola and Muniasamy Neerathilingam
}

\begin{abstract}
Aptamers are synthetic ssDNA/RNA molecules that are emerging as novel tools for the development of therapeutics, especially for targeted delivery. Aptamers are comparable to monoclonal antibodies, which are well-established therapeutic molecules, in terms of specificity and affinity to their target. The advantage of aptamers over antibodies includes their high stability, ease of synthesis, less batchto-batch variation, easy chemical modifications that allow different conjugation chemistries, small size for better tissue penetration and low immunogenicity. These advantages make aptamers an important tool for use in therapeutics for targeted delivery. However, aptamers do have some limitations that have hindered their widespread clinical use as a therapeutic agent. Some of their common limitations include serum stability, renal filtration and endocytic escape. Other limitations that are more specific to aptamers include lack of diversity in the aptamer library, nuclease susceptibility and claims of aptamer specificity as well. This book chapter sheds light on these challenges, and using examples, it explains the scientific advancements that have been achieved in overcoming these limitations. We will end this chapter by discussing the use of high-throughput technology, which is the only way of truly industrializing the aptamer technology akin to the development of small molecule drugs.
\end{abstract}

Keywords: aptamers, SELEX, targeted delivery, serum stability, endosomal escape, renal clearance, high-throughput

\section{Introduction}

Aptamers are small single-stranded RNA or DNA oligonucleotides that specifically bind to their target due to their unique 3-dimensional structure. They were first independently developed by the groups of Gold and Szostak in 1990 [1, 2]. Aptamers are selected from a pool of random oligonucleotide library $\left(>10^{15}\right.$ random sequences) by iterative rounds of selection and amplification by a process called Systematic Evolution of Ligands by EXponential Enrichment (SELEX). Different intermolecular interactions facilitate the interaction of aptamers with their target including van der Waal's forces, electrostatic interactions between charged groups, three-dimensional shape, stacking and hydrogen bonds.

Functionally aptamers are same as antibodies; however, their advantages over antibodies include small size, less immunogenicity, ease of synthesis, easy chemical 
modification that allows conjugation with a variety of molecules and stability at a higher temperature. Aptamer is comparably smaller $(\sim 3 \mathrm{~nm})$ than an antibody $(10-15 \mathrm{~nm})$ [3], thus, allowing higher penetration in tissues and the ability to bind more dense cellular epitopes in living cells. Unlike antibodies, aptamers can be selected against non-immunogenic molecules, including proteins or peptides as well as toxins. Aptamers have been successfully developed against proteins, peptides, dyes, metal ions, viruses, bacteria, toxins, and whole cells. They have high specificity and affinity often comparable with antibodies. Unlike antibodies, they can be selected at non-physiological conditions such as extremely high or low temperature, or $\mathrm{pH}$. Synthetic manufacturing of aptamers allows minimal batch-to-batch variation, which is a tedious task to maintain in antibody development. All these properties make aptamers as suitable candidates for therapeutic use, particularly targeted nano-delivery. Several aptamers have already been selected against cell surface targets for targeted delivery of therapeutic payload for different diseases, e.g., cancer and human immunodeficiency virus (HIV) infected cells [4]. Table 1 lists the common differences between antibodies and aptamers.

In this book chapter, we discuss the challenges that hinder the clinical use of aptamers as targeted delivery molecules. For example, the small size of aptamers is like a double-edged sword. Though it helps in higher tissue penetration, but also results in high renal filtration that causes loss of its therapeutic efficiency. Using examples we show that how challenges in serum stability, renal filtration, selection

\begin{tabular}{|c|c|c|}
\hline & Antibody (IgG) & Aptamer \\
\hline Size & $10-15 \mathrm{~nm}$ & $\sim 3 \mathrm{~nm}$ \\
\hline Target & $\begin{array}{l}\text { Only immunogenic targets. Can't } \\
\text { be raised against toxic or non- } \\
\text { immunogenic proteins }\end{array}$ & $\begin{array}{l}\text { Produced against immunogenic and non- } \\
\text { immunogenic (e.g., metal ions, dyes, small } \\
\text { peptides, toxins etc.) targets }\end{array}$ \\
\hline Specificity & High & High \\
\hline Affinity & High & High \\
\hline Penetration & $\begin{array}{l}\text { Low tissue penetration due to } \\
\text { large size }\end{array}$ & High tissue penetration due to small size \\
\hline $\begin{array}{l}\text { Stability (pH, } \\
\text { Temperature) }\end{array}$ & Low & High \\
\hline Shelf life & $\begin{array}{l}\text { Few months (at low temperature; } \\
\text { repeated freeze-thaw causes } \\
\text { loss-of function) }\end{array}$ & $\begin{array}{l}\text { Several months (at room temperature) to } \\
\text { several years (frozen) }\end{array}$ \\
\hline $\begin{array}{l}\text { Nuclease } \\
\text { susceptibility }\end{array}$ & Absent & $\begin{array}{l}\text { Present (modified nucleotides minimize } \\
\text { nuclease susceptibility) }\end{array}$ \\
\hline Immune response & High (except humanized Ab) & Absent or low (rare cases) \\
\hline $\begin{array}{l}\text { Batch-to-batch } \\
\text { variation }\end{array}$ & Present & Absent due to synthetic manufacture \\
\hline Conjugation & Less possibilities & $\begin{array}{l}\text { Easy conjugation due to chemical nature } \\
\text { allows functionalization with a wide variety } \\
\text { of molecules }\end{array}$ \\
\hline Synthesis & Only in physiological conditions & Synthetic \\
\hline Cost of synthesis & $\begin{array}{l}\text { High. Requires animal house } \\
\text { facility or reactors }\end{array}$ & $\begin{array}{l}\text { Low. Synthesized using table-top } \\
\text { instruments }\end{array}$ \\
\hline Scale-of-synthesis & Low & Scalable \\
\hline
\end{tabular}

Table 1.

This table lists the common differences between an antibody $(\operatorname{IgG})$ and an aptamer. 
methodology and endocytic escape have been overcome. We explain the importance of proper controls and validation methods in SELEX methodology, which would otherwise result in the development of poor quality aptamers and, this might ultimately diminish the confidence in this technology. Lastly, we discuss about the development of automation in SELEX technology, an important step toward industrializing the aptamer selection process.

\section{Serum stability}

Currently, many aptamers are in different stages of clinical trials for various diseases [5]. Most of the drugs are administered systemically, i.e., intravenously. This method poses a challenge of overcoming serum stability, which involves overcoming nuclease activity and coagulation. Here in we discuss these two topics in detail:

\subsection{Aggregation}

Aptamers have been used to functionalize liposomes to increase their bioavailability through targeted delivery (Figure 1) [4]. Since aptamers are negatively charged it is easier to pack them with cationic liposomes. However, serum proteins being charged molecules bind to cationic lipids, thus, lowering their targeting efficiency due to change in structure, aggregation or dissociation of the aptamercationic lipid complex [6,7-9]. Polycationic lipids have also shown cyto(toxic) effects [10]. The problem of aggregation incurred by the charge of cationic lipids can be overcome by using neutral helper lipids $[11,12]$. But another challenge is that lipid conjugated aptamers may also bind to erythrocytes, and filtered out through liver and spleen. For example, 1,2-dioleoyl phosphatidylethanolamine (DOPE), a neutral helper lipid, binds to erythrocytes. As an alternative, another neutral helper lipid, cholesterol, may be used which shows significantly lower binding to erythrocytes as compared to DOPE $[10,13]$.

Cholesterol conjugated RNA aptamer against Hepatitis C virus (HCV) NS5B protein has been used to inhibit HCV replication in human liver cells without induction of cytotoxicity in vitro. Next, in this study, the same molecule was injected in a mouse model but found no induction of innate immunity. The half-life of the cholesterol-conjugated aptamer was longer than that of the

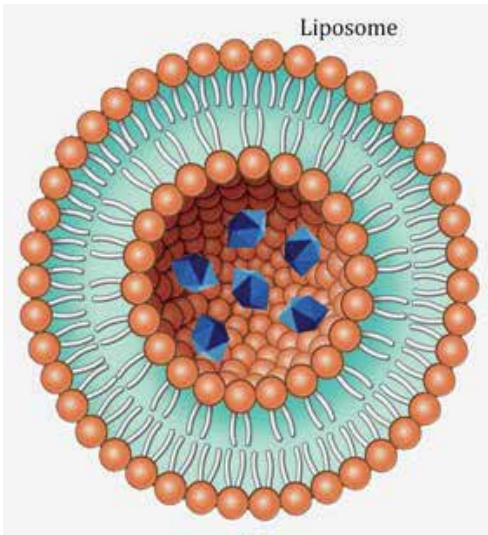

(a)

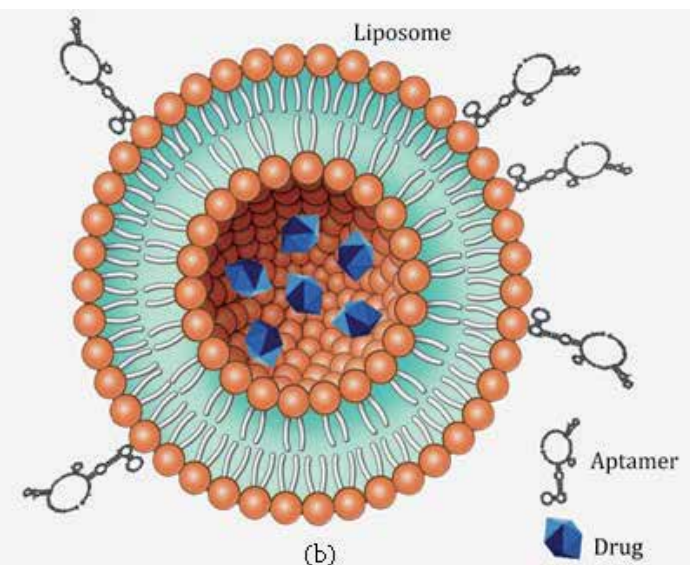

(b)

Figure 1.

Illustration showing (a) non-functionalized liposome and (b) aptamer-functionalized liposome carrying drug molecules. 
non-cholesterol-conjugated aptamer, and in accordance, cholesterol conjugation reduced the clearance by 9 -fold [14].

It is important to note that no one lipid composition induces cytotoxicity in all cell types, i.e., a conjugated lipid may be toxic to one cell type but not to another. Improvements of lipid formulations are constantly explored by the addition of different lipids, targeting molecules, or shielding moieties designed to prevent clearance in vivo. Use of neutral lipids for aptamer administration has been explored less due to the difficulty in loading hydrophilic aptamer molecules in the neutral lipids for formulation, as compared to (poly)cationic lipids. Liposomes are just one tool for enhancing the efficacy of aptamers for targeted delivery, and there are multitudes of vehicles that are being examined.

\subsection{Nuclease activity}

Nuclease activity of the serum is helpful in cleaving foreign DNA, thus, it is a way of prevention from pathogens. However, this activity is deleterious to the oligonucleotide-based therapeutics, including aptamers. Unmodified aptamers may have very short half-lives in vivo ( $<10$ minutes) [15]. Two types of nucleases act on these aptamers, viz., exonucleases and endonucleases. Hence, different strategies have been designed to prevent the cleavage activity of each of the nucleases.

In-SELEX and post-SELEX strategies can be utilized to develop nuclease resistant aptamers. Each of these two strategies has its own advantage and limitation. We discuss these two below:

\subsubsection{In-SELEX}

In this methodology aptamers with the desired modification are generated during the aptamer selection process using modified nucleotides, e.g., modified $2^{\prime}$ sugar position using $2^{\prime}$-amino pyrimidine nucleosides [16, 17], 2' -fluoropyrimidine nucleosides [18, 19], 2'-O-methyl purine [20], and 2'-O-methyl pyrimidine nucleosides [21, 22] and locked nucleic acids (LNAs) [23, 24] have been commonly used for this purpose, preventing resistance from endonucleases. Macugen, the only FDA approved therapeutic aptamer, was also selected using this approach [25]. The Advantage of this methodology is that no further modification is required in the selected aptamers, thus, ruling out the possibility of loss of function due to post-SELEX modification. However, use of unnatural nucleotides posed a challenge to early researchers since wild-type DNA/RNA polymerases could not amplify the unnatural aptamers during preparative PCR round of aptamer selection. However, using synthetic biology researchers developed new polymerases that could amplify oligos with unnatural nucleotides. Still, amplification using these mutant polymerases is not comparable with natural polymerases; hence, aptamer library amplification is a bit challenging.

SOMAmers are another class of aptamers that bear $\mathrm{dU}$ residues at the $5^{\prime}$-position. These are uniformly functionalized at the $5^{\prime}$-position with various moieties (e.g., benzyl, 2-naphthyl, or 3-indolyl-carboxamide). These moieties can interact with the target molecule and form novel secondary and tertiary structural motifs within the SOMAmer. Introduction of dU at the $5^{\prime}$-position offers nuclease resistance to these molecules [26].

\subsubsection{Post-SELEX}

Post-SELEX involves the introduction of desired modification in pre-selected aptamers during solid-phase chemical synthesis. This method may often lead 
to reduced affinity or specificity of the aptamers due to the structural change in the aptamer that is brought upon by the modification. Hence, a combination of methods has to be tested on the aptamer, and one that renders no loss of function has to be selected. Modification of $5^{\prime}$ and $3^{\prime}$ nucleotides is most common and effective in this case. Sometimes unmodified aptamers may demonstrate high resistance to serum nuclease activity [27]. This feature might develop due to the unique 3-D conformation of the aptamer that protects the $5^{\prime}$ - and $3^{\prime}$-termini from exonucleases.

Spiegelmers are excellent nuclease resistant $L$-form RNA or DNA aptamers that are chiral inversions of the natural D-forms. Since naturally occurring nucleases can act only on $D$-form of nucleotides, they are very less likely to degrade the $L$-form spiegelmers [28]. Also, due to their unnatural conformation, they are less likely to generate an immune response. The unnatural conformation of spiegelmers also renders it unfit for processing by polymerases for amplification during SELEX process. Hence, in the first step, an aptamer library of natural D-oligonucleotides is selected against a synthetic enantiomer of the $L$-protein target. After the aptamer selection is done, aptamers are converted from $D$-form to $L$-form. As per the rules of symmetry, the resulting $L$-aptamers (spiegelmers) would bind to the natural $D$-protein target with the same affinity as the $D$-aptamers bind to the mirror image selection target [29].

\section{Diversity of aptamer library}

As we know, aptamer binding to its target depends on its 3-dimensional conformation, which is ultimately governed by its sequence. Hence, an aptamer library with a high degree of sequence variability will have a high variety of structurally variant aptamers; therefore, such a library will have a higher degree of success in developing tight binding aptamers to its target. In contrast to proteins, which are made of 20 different amino acids due to a variety of functional groups, nucleic acids possess a very limited variety of functional groups, i.e., only four bases (adenine, guanine, cytosine and thymine/uracil). Thus, aptamers can be endowed with protein-like properties by addition of functional groups that mimic amino acid side-chains to expand their chemical diversity [26].

SomaLogic, a leading company in aptamer development, pioneered in increasing the repertoire of aptamer sequences in a library. They introduced new functional groups, i.e., hydrophobic side chains (e.g., naphthyl, benzyl, tryptamino and isobutyl) at 5'-position of dUTP nucleotide. Aptamers developed using these modified nucleotides were called Slow Off-rate Modified Aptamers (SOMAmers), and they showed higher success rate in aptamer selection against difficult proteins with success rate going from $<30 \%$ for unmodified aptamers to $>90 \%$ for SOMAmers [30].

Kimoto et al. used an extra base pair Ds:Px in the initial library to introduce variety, and developed aptamers with 100-fold higher affinity as compared to the unmodified analogs against VEGF-165 (vascular endothelial growth factor) [31]. Sefah et al. developed introduced non-natural nucleosides $\mathrm{Z}$ and $\mathrm{P}$ to develop aptamers specifically targeting liver cancer cells [32]. In another work, glycosylated aptamers were used in a method called SELection with Modified Aptamers (SELMA) to develop low-nanomolar affinity aptamers against HIV broadly neutralizing antibody 2 G12 [33]. These antibodies protect against HIV by recognizing the unique carbohydrate epitope on HIV. Here, the glycosylated aptamers worked as a scaffold that presented carbohydrate in a manner that mimics their multivalent presentation on HIV, thus it could be used as a vaccine component. 


\section{Renal filtration}

Renal filtration of small molecule drugs is a phenomenon that needs to be overcome for efficient therapeutic use. Owing to their small size, aptamers also undergo this challenge. An aptamer of 6-30 kDa mass has a size of $<5 \mathrm{~nm}$ [34]. When an unmodified aptamer is administered intravenously, even using stabilizing backbone modifications, they are subjected to rapid excretion through renal filtration, hence, reduced circulation time. To overcome this challenge, aptamers are functionalized with bulky moieties viz., polyethylene glycol (PEG), liposomes, proteins, cholesterol, organic or inorganic nanomaterials, or multimerized to reach a mass above the threshold of glomerulus cut-off $(30-50 \mathrm{kDa})$ [34].

\subsection{PEG attachment}

PEGylation is one of the most common methods to prevent renal filtration of aptamers. PEG is a flexible, uncharged and a highly hydrophilic polymer that is widely conjugated with therapeutic drugs to reduce reticuloendothelial clearance, extend circulation time and improve drug efficiency [35]. Macugen, which is the only FDA approved aptamer drug in the market is also PEGylated. PEG decreases aggregation and increases the solubility of the conjugates. PEGylation of Macugen increased its half-life to $9.5-12.5$ hours after intravenous and subcutaneous injection, respectively, in the plasma, and up to 94 hours in vitreous humor [36, 37]. Similarly, PEGylation also increased in vivo half-life of M7, a DNA aptamer, from $<1$ to 24-48 hours [38]. This modification did not cause any change in the specificity of M7 aptamer toward its target PD-1 (Programmed death protein 1) and suppressed the growth of PD-L1 (Programmed death-ligand 1) positive colon cancer carcinoma in vivo [39].

\subsection{Cholesterol}

A 29 nucleotide-log 2'-F pyrimidine modified RNA aptamer was reported to inhibit Hepatitis $\mathrm{C}$ virus (HCV) replication in vitro. In the follow-up study, this aptamer was derivatized with cholesterol to form cholesterol aptamer conjugate (chol-aptamer). This conjugate entered the cell and inhibited HCV RNA replication in a cell-based system successfully. Systemic administration of chol-aptamer was well tolerated by mice and increased retention time in plasma [14].

\subsection{Dialkyl lipids}

Willis et al. conjugated diacylglycerol (DAG) to the $5^{\prime}$-end of a nuclease resistant VEGF aptamer [40]. This DAG-aptamer conjugate was then incorporated to a liposome bilayer, which resulted in aptamers with improved anti-VEGF activity in vitro and in vivo, both. Importantly, this DAG-aptamer-liposome complex had a considerably better residence time in plasma as compared to unmodified aptamer [40].

\section{Toxicity}

Toxicity, side effects, and immunogenicity are one of the most important criteria in drug evaluation, apart from shelf life and efficacy of the drug. Aptamers have a significant advantage over antibodies due to their minimal or non-immunogenic nature. Early preclinical studies using Pegaptanib sodium (Macugen), the aptamer drug for preventing wet age related macular degeneration (AMD), did not exhibit any intrinsic toxicity during preclinical studies [37, 41, 42]. The only side effect 
reported arose almost exclusively due to the injection procedure instead of the drug itself [43]. Similarly, other works have also described aptamers as non-toxic therapeutic agents $[44,45]$.

Though aptamers may be inherently non-immunogenic, chemical modification or conjugation may render them immunogenic or cytotoxic. Hence, chemical modification of aptamers works as a double-edged sword. For example, LNAmodified nucleic acids have shown severe hepatotoxicity [46]. 2 '-fluoro pyrimidine and $2^{\prime}$-orthomethyl pyrimidine modifications are commonly used in aptamers to introduce endonuclease resistance. 2'-fluoropyrimidine modified RNA aptamers lead to cytotoxicity and induction of interferon-beta expression in human cancer cells in vitro. Interestingly, 2 '-orthomethyl pyrimidine completely abrogated the induction of cell death and cytokine expression in the same cells [47].

There is a limited toxicological data available on aptamers. Mainly because the field is still in its initial stage and the majority of aptamer-based drugs are still under clinical trials. In comparison, a significant amount of toxicological data is available for antisense oligonucleotides (ASOs). Some cues might be taken from toxicity studies on ASOs; however, we must remember that aptamers are structurally a different class of oligonucleotides; hence, they may not behave in exactly the same way as ASOs. Toxicological properties of ASOs have been described in earlier reviews [48-50]. Less information is available on other class of immunostimulatory oligonucleotides viz., siRNA, gene therapy, and other partially or completely nucleic acid-based molecules. Three major oligonucleotide-based class effects that have been described are: polyanionic effect, the stimulation of innate immunity, and tissue accumulation of oligonucleotide material [51]. These class effects are briefly described below.

Polyanionic effects occur due to the non-specific off-target interaction with proteins that may affect protein function and it usually occurs at a high concentration of ASOs. The toxicological effects of polyanionic effect include activation of the alternative pathway of complement $\left(\mathrm{C}^{\prime}\right)$ that results in pseudohypersensitivity responses and inhibition of the coagulation pathway, thus, resulting in delayed clotting. Complement activation has been studied predominantly in non-human primates, and to some extent in humans. Complement activation is a thresholddependent phenomenon and will occur acutely once the threshold is crossed [52].

Stimulation of innate immunity has been reported for ASOs. This activation occurs due to the interaction of Toll-Like Receptor 9 (TLR 9) with the CpG motif within the single-stranded DNA. When CpG motifs are unmethylated, they act as immunostimulants [53]. Unmethylated CpG motifs are common in microbial genomes but occur quite less in vertebrate genome, hence they are called as pathogen-associated molecular patterns (PAMPs) [54]. CpG PAMP is recognized by pattern recognition receptor TLR9, which is expressed constitutively by B cells and plasmacytoid dendritic cells (pDCs) in humans and other higher primates [55].

Lastly, oligonucleotide accumulation is observed in certain tissues and cells. This accumulated material is termed basophilic granulation, and it is observed most commonly in renal proximal tubular epithelium and tissue macrophages. Accumulation of basophilic granules usually has no effect on associated cells; however, its accumulation at a very high level in cells, especially renal proximal tubular epithelium, may lead to cytotoxicity and eventually organ dysfunction [56].

Apart from the above mentioned three oligonucleotide class effects, there are other effects that should also be studied; however, these three are the most commonly observed and, hence, should be assessed carefully. In a very interesting work, Guo et al. demonstrated that RNA molecules of different shape, size or sequence can initiate immunogenicity at different levels in vitro and in vivo, both. Findings from this work suggest that properties of RNA nanoparticles may be tuned for safe 
use as therapeutic nanocarriers [57]. Findings from this work may be extrapolated to RNA aptamers to some extent.

Conjugated moiety may also cause an adverse affect in aptamer drug. For example, penivacogin, a PEGylated aptamer drug, induced severe allergic reaction during first exposure to the drug during phase III clinical trials. The allergy occurred due to pre-existing anti-PEG antibodies [58]. PEG is widely used in several consumer products including topical and parenteral medications. Possibly, exposure to these wide varieties of products leads to the development of anti-PEG antibodies [35].

Although less clinical data is available to assess the cytotoxicity of aptamers, studies in animal models show low or no cardiotoxicity, hepatotoxicity or renal toxicity. Doxorubicin (DOX), a widely used anticancer drug causes cardiotoxicity as a side-effect due to non-specific uptake. Neither liposomal DOX nor PEGylated DOX has resolved this issue. However, aptamer-DOX conjugate is known to reduce this cardiotoxicity along with increase in efficacy toward tumor suppression in comparison to non-targeted delivery [59] in vivo.

The hepatic uptake of aptamer functionalized gold nanostars (Apt-Au NS) was studied by Dam et al. They found high accumulation of the complex in liver; however, there was no hepatic acute toxicity. This was probably due to the high accumulation of the complex in macrophages instead of the hepatocytes [60]. Similarly, other works also demonstrated lack of hepatic and renal toxicity in vivo for aptamer-mediated tumor suppression $[45,61]$.

\section{Selection process}

Aptamers are selected from a pool of random single-stranded DNA/RNA oligonucleotide library ( $>10^{15}$ random sequences) by iterative rounds of selection and amplification by SELEX method as shown in Figure 2 [4]. Therapeutic aptamers have been developed against cell surface targets [62] as well as for delivery of therapeutic conjugates into the cells, i.e., for internalization [3]. Though the selection method is fundamentally the same for developing aptamers for either use, the cell-internalizing aptamers require more points to be addressed to ensure a higher degree of success. Endosomal escape and effective delivery to the cytosol is an important aspect that should be considered for the effective biological activity of a cargo. Here we describe current selection methods commonly used for selection of cell-internalizing aptamers, and discuss the limitations of each method. For this purpose, here we compare the two commonly used strategies, i.e., SELEX and cell-SELEX.

\subsection{SELEX}

Aptamers have been historically selected against cell surface biomarkers using purified or recombinant proteins. Majority of aptamer therapeutics that are currently in clinical trials have been selected using this methodology. However, there are problems associated with this selection method since the protein against which selection is done might not be in its natural conformation. Proteins are present in physiological milieu in a unique 3D conformation due to either posttranslational modification or their association with other interacting proteins. Proteins that are produced in vitro usually don't possess these post-translational modifications, specifically, if they produced in a prokaryotic system, e.g., commonly used E. coli. Hence, it is advisable to use eukaryotic expression system viz., mammalian, yeast or insect cells as they would possess post-translational modifications which may 


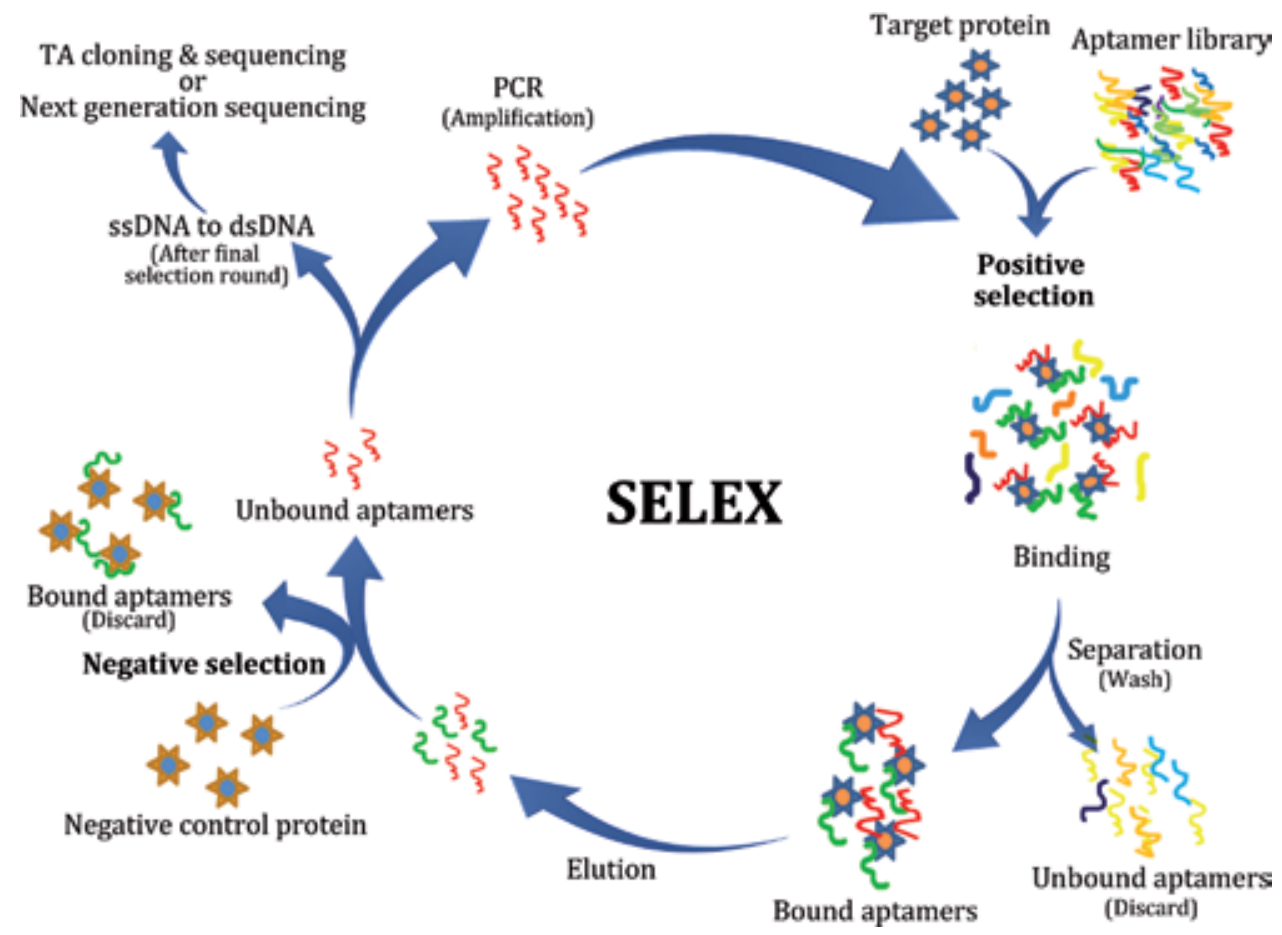

Figure 2.

Diagrammatic representation of the systematic evolution of ligands by EXponential enrichment (SELEX) method. A library of $\sim 10^{15}$ different single-stranded DNA/RNA molecules is incubated with the target molecules followed by washing. Unbound sequences (non-specific aptamers) are discarded. Bound sequences are recovered and added to the negative control target. Here, the bound sequences (these are aptamers binding to common epitopes) are discarded, and the unbound sequences (target-specific aptamers) are recovered. These sequences are amplified and subjected to further rounds of in vitro selection followed by sequencing after the final selection round. The figure is adapted from Chandola et al. [4].

be crucial for the correct 3D conformation for selection of aptamers. However, low protein yield might be an issue if we chose a eukaryotic expression system over the prokaryotic one. There is also a possibility that recombinant cell surface proteins may be insoluble since they need interaction with other cell components (e.g., G-protein coupled receptor), or form multimeric and/or multivalent structures $[63,46]$. Lastly, in the majority of cases, aptamer selection is done using only the extracellular domain of the target protein to avoid aptamer selection against the intracellular region of the protein; however, the absence of transmembrane domain may again alter the natural conformation of the protein. Therefore, there is a possibility that aptamers selected using protein-based SELEX approach might not be able to bind their target in a physiological milieu. Liu et al. selected RNA aptamers against histidine-tagged epidermal growth factor receptor variant III (EGFRvIII) ectodomain. This receptor is present in glioblastoma, but not in normal brain tissue. This protein was expressed in an E. coli expression system, hence, lacked a specific post-translational modification, i.e., glycosylation. Lack of glycosylation at this particular site resulted in significant alteration of EGFRvIII protein structure and, hence, the selected aptamer was unable to bind this receptor protein in target cells [64]. Using recombinant protein with a tag-normally used for affinity column based purification of a recombinant protein, e.g., His tag-may result in non-specific aptamer selection against the protein tag; hence, it is always recommended to cleave the tag before a purified recombinant protein is used for aptamer selection. Lastly, attachment of a protein to the matrix may result in non-specific aptamer selection against the matrix itself. 


\subsection{Cell SELEX}

Above mentioned limitations, viz., altered 3D conformation, undesired aptamer selection against the protein-tag or matrix can be overcome by using cell-SELEX methodology. In cell-SELEX aptamer selection is performed on live cells in vitro, hence, target surface proteins are present in their native conformation. Unlike protein-based SELEX, cell-SELEX does not need any prior information about the native conformation or biological function of the target protein. This method relies on the difference in the expression pattern of cell surface receptors between the diseased target cells (e.g., cancer cells) and the non-diseased cells (e.g., healthy cells). The aptamer selection is first performed on a negative control, i.e., healthy cells. From here, the unbound aptamers are collected and added to the test cell line, i.e., diseased cells. Only the unbound aptamers are collected from negative control cells because they are uncommon among the target and control cells, and it possibly has a set of aptamers that bind the upregulated cell surface receptors that are present only on target diseased cells. Using the above-mentioned methodology, cell-SELEX has been used to identify novel tumor biomarkers [65]. In our experience, major challenge of cell-SELEX is the optimization of aptamer enrichment by PCR as compared to protein-based SELEX. This is due to the inhibitors or other cellular contaminants (e.g., DNA or RNA) that come in the aptamer pool which inhibit the polymerasebased PCR reaction for enriching aptamer pool during each round of selection. Also, lack of prior knowledge about the identity and expression level of the biomarker may lead to aptamer selection against unrelated/unwanted off-target surface molecules that are co-expressed on target cells; hence, more rounds of counter selection with the control cell line is required to improve the selectivity of aptamers.

An ideal aptamer candidate for internalization purpose should bind to a cell surface receptor that is present in high quantity, and it should have a high rate of endocytosis upon binding of a ligand. However, aptamers selected by cell-SELEX may not fall in this category always. Hence, researchers have developed novel strategies to facilitate enrichment of cell internalizing aptamers by alteration of the traditional cell-SELEX protocol. For this purpose, Thiel et al. developed a methodology called cell-internalization SELEX. Here, target cells were incubated with an RNA library followed by high-salt wash [66]. This method eliminates the non-internalizing surface bound aptamers or those that internalize at a slower rate. Cell internalizing aptamer against Human epidermal growth factor receptor (HER2), a transmembrane protein overexpressed in breast cancer cells was selected using this method [66]. Later studies reported that a high-salt wash is insufficient to remove the non-internalizing surface bound aptamers. Hence, Levy et al. (2012) used a cocktail of RNAses to digest the cell surface bound non-internalizing aptamers $[67,68]$. Using this strategy, this group selected cell internalizing 2 '-fluoropyrimidine RNA aptamer called c2 against human transferrin receptor (hTfR).

Proteinase $\mathrm{K}$ is a broad-spectrum serine protease that cleaves peptide bond adjacent to the carboxyl group of aliphatic and aromatic amino acids with blocked alpha-amino groups. Proteinase $\mathrm{K}$ was used as an alternate approach to digest protein domains present on the cell surface after incubation with aptamer library. Thus, digestion of protein domains would render loss of cognate epitopes needed for aptamer binding. After proteinase $\mathrm{K}$ treatment and washing of the cells, the aptamers non-specifically bound to the cell surface proteins would be removed, and only those which have internalized the cells can be obtained, as desired. Proteinase K treatment can be used for DNA and RNA aptamers both, unlike RNase treatment used by Levy et al. [68] which is applicable only for RNA aptamers. Iaboni et al. used this method to select cell-internalizing aptamers against insulin receptors on human glioma cells [69]. 


\section{Strategies for endosomal escape of cargoes}

The therapeutic efficiency of a cell-internalizing platform depends on its ability to escape from endosomes and deliver the therapeutic cargo to the cytoplasm. The efficiency of endosomal escape, which is $0.01 \%$ for traditional delivery systems, should be increased significantly to achieve higher therapeutic efficacy [70]. Two types of strategies are commonly employed to facilitate endosomal escape. First method uses endosomal destabilizing agents that alter the permeability of endosomal membranes, e.g., chloroquine. However, the limitation of this method is that these endosomolytic agents may reach lysosomes, thus, lysing them and lead to cytotoxicity due to the release of lysosomal lytic enzymes. Second method, which is found to be non-toxic in several studies, uses engineered viral or bacterial cell penetrating peptides (CPPs) (e.g., truncated HIV-1 TAT peptide or Aurein1,2) [71-73]. However, CPPs can increase the escape of cargoes from endosomes to the cytoplasm by only 5 - to 8 -fold, which is not enough to generate an effective platform for clinical applications [70]. Below we will discuss the strategies that could be used with aptamers to enhance their endosomal escape properties or that of the attached cargo.

Any targeted delivery platform for internalization of a cargo to cells is endocytosed by receptor-mediated endocytosis (RME) to a large extent [74], and aptamers fall in this category. The endocytosed cargo first reaches early endosomes, from where it is either sent back to cell surface (recycling endosomes) or sent to late endosomes, and finally lysosomes. The cargo may be degraded by hydrolytic enzymes in lysosomes. Therefore, if a cargo enters an endosome that is destined for lysosomes, it has a short period of time within which it has to escape to the cytosol to prevent its degradation. Different strategies have been developed that can be used in conjugation with aptamers, which may help in their endosomal escape. Endosomal sorting complex required for transport-I (ESCRT-I) is a protein complex that plays an important role in maturation and trafficking of endosomal cargoes. Wagenaar et al. silenced two components of ESCRT-1 (i.e., TSG101 and VPS28) to induce endosomal escape of delivered anti-miRNA in vitro and in vivo, both, and resulted in tumor regression [75].

Nieman-Pick type C1 (NPC1) is a late endosomal/lysosomal membrane protein required for extracellular recycling of endosomal contents. A small molecule inhibitor of NPC1 protein, termed NP3.47, was earlier used to delay maturation of non-recycling endosomes [76]. The Study found that NP3.47 caused 3-fold higher accumulation of lipid nanoparticle (LNP) formulations of siRNA, i.e., LNP-siRNA as compared to the controls in late endosomes/lysosomes in a variety of cell lines causing 4-fold higher gene silencing. Authors proposed that trapping LNP-siRNA in late endosomes extended the window of time for a more efficient escape into the cytosol to facilitate siRNA induced gene silencing [76].

$\mathrm{pH}$-dependent cargo release is also an effective way for endosomal escape. Anti-PSMA aptamer-siRNA chimera was conjugated to a $\mathrm{pH}$-dependent polyhistidine domain via dsRNA binding domain (dsRBD). In the acidic compartment of a mature endosome $(\mathrm{pH} \leq 6)$, Histidine becomes protonated and facilitates osmotic swelling that leads to cargo release into the cytosol, a mechanism known as the proton sponge effect [77]. By this mechanism, the polyhistidine tag facilitated the escape of anti-PSMA aptamer-siRNA chimera from late endosome to cytosol resulting in improved silencing of the target gene [78].

Another straightforward strategy for cytoplasmic delivery is to perform aptamer selection against a cell surface target protein that internalizes to other compartments by receptor-mediated endocytosis. RME facilitates more rapid internalization of the targeting moiety as compared to untargeted complexes. Depending on 
the intracellular receptor-dependent or independent trafficking path, the fate of targeting moiety can be controlled, e.g., macromolecules internalized by clathrindependent RME are mostly destined for lysosomal degradation whereas clathrinindependent internalization leads to endosomal accumulation and sorting to a non-degradative path [74]. However, this method can be used against those targets that have previously been characterized, e.g., nucleolin. Nucleolin is a nuclear ribonucleoprotein that is overexpressed in cancer cells where it shuttles among the cell-surface, cytoplasm and the nucleus. AS1411 aptamer, a G-quadruplex forming oligonucleotide, specifically binds nucleolin and displays antiproliferative activity against cancer cells [79]. However, there are conflicting reports regarding nucleolinmediated uptake of AS1411 since one study suggest that it is nucleolin dependent and the other suggests that it is nucleolin independent. Though the mechanism of escape of AS1411 from endosomes to cytoplasm is unclear, it is likely that its interaction with nucleolin, which shuttles between different cellular compartments, plays a role in it [80].

\section{Quality of known aptamers}

Aptamers have generated excitement in the scientific community in the last 2 decades due to their unique characteristics that helped them gain popularity for use in therapeutic purposes. They have been used as both agonists and antagonists targeting cell surface receptors [34]. The Ability of aptamers to specifically bind the cell surface targets catapulted them into the field of targeted delivery of therapeutics. They can be easily conjugated with small molecule drugs, liposomes, proteins, siRNAs, miRNAs, or other nanoparticles. However, when industry leaders in the field of siRNA/miRNA delivery tried to recapitulate the results of siRNA delivery, they failed [81]. Also, since the approval of Macugen, in 2004, by Food and Drug Administration (FDA), no other aptamer-based drug has made to the market. Failure of Fovista (anti-PDGF aptamer to prevent age-related macular degeneration) and Reg1 (an antifactor IXa aptamer paired with its complementary reversal agent, reverseran, to restore blood clotting) failed in phase III clinical trials due to no improvement over current standard care methods has also been disappointing [81]. However, Reg1 also resulted in serious allergies in patients, albeit it was due to conjugated PEG, and not due to the aptamer itself. Though failure of drugs in clinical trials is quite common as many more drugs fail during trials than pass them, but more disappointingly, there is a lack of aptamer-based drugs that are even advancing to the clinic [5]. Unlike antibodies and antibody-based drugs which are supported by big pharmaceutical companies, aptamer development has been largely relegated to academic labs that are often, comparatively, poorly funded.

Levy group observed lack of reproducibility in aptamer binding to its target or therapeutic effects in several cases [81]. This unreliability among novel molecules is a bad precedent that may generate a lack of confidence in an upcoming technology. The poor reproducibility, or robustness, of these aptamers might be due to poor downstream characterizations, which is, in fact, very important for validation of novel aptamers. There is also no standard way of assessing aptamer binding results, as different labs use a variety of methods to assess the binding function of their selected molecules. Hence, there is no straightforward way to compare or validate results between different labs. Other labs often cite the work from previous labs without revalidating the aptamer that they intend to use. Lack of funds, resources or time are often the reason nor not revalidating the aptamers for their own purposes; however, if the aptamer field has to grow, a bit more care and diligence will be needed from the labs that are developing aptamers as well as the end user. 
Here, we briefly discuss the validations and controls that should be used to ensure a more reliable selection of aptamers for targeted delivery. Soluble proteins or peptides are often used as targets for aptamer selection, and their binding affinity or specificity is confirmed by binding assays. However, further validation using a negative control cell line or a knockout cell line is quite important for such aptamers since aptamers may be selected against an epitope that is buried in the plasma membrane or it might be located in the cytoplasmic region.

Varieties of assays are performed to study aptamer binding to target cells, e.g., radioactively or fluorescently labeled aptamers. However, limitation of these assays is that they are performed on bulk cells, hence, it is not known whether the observed signal is due to interaction with the entire cell population, or only a subset of cells. Confocal imaging is often used as a qualitative method to confirm internalization of aptamers, or localization in a particular organelle. Quantification of the signal and statistical methods are often not performed. Since a very small number of cells are typically interrogated, statistical analysis is not performed [81]. The low throughput of imaging, i.e., low cell counting in confocal imaging is usually due to strong lasers that may bleach the fluorescent labeled aptamers. However, with the advent of LED-based high content imaging systems, quantitative analysis in a high-throughput fashion can be easily performed. It is recommended that aptamers should be conjugated to high-quality fluorophores in these studies, i.e., Alexa Fluor dyes or quantum dots instead of traditionally used FITC or TRITC, as these are less resistant to photobleaching. Flow cytometry is also highly recommended to study aptamer internalization due to its high sensitivity, and its ability to interrogate each cell in a population individually.

It is advisable to limit the incubation time with aptamers during cell-internalization SELEX, typically up to $\sim 1$ hour. Incubation with cells for an extended period of time may lead to significant levels of non-specific uptake. It is also recommended to use blocking agents to prevent non-specific binding of aptamers to cell surface. Aptamers are polyanions, therefore, they can readily interact with cell surface moieties due to charge-charge interactions, especially at high concentrations $(>1 \mu \mathrm{M})$. A Nonspecific polyanionic competitor like ssDNA or tRNA can be used successfully to significantly inhibit non-specific aptamer binding to cells [81]. As an alternative, dextran sulfate may also be used for the same purpose [5].

\section{Automation of SELEX protocol}

Aptamer selection is a repetitive, time-consuming and a laborious process. The immense number of PCR reactions during preparative PCR step for the enrichment of selected aptamers is also a monotonous process which might lead to manual errors. Various tasks in molecular biology such as DNA sequencing, plasmid preparation, and microarray construction have been automated [82, 83]. The automation of these processes has substantially increased the throughput of sample preparation or assays and significantly reduced the amount of time a researcher must spend to perform purely repetitive mechanical tasks.

Automation reduces variability during the selection process, leading to better reproducibility of results. Depending on the degree of automation, manpower, time and consumables can be reduced, whereas throughput can be increased by parallelization. The extent of lab automation depends on scope and timeline of the project, and the allocated budget [84]. In principle, there are two concepts of automation, viz., full automation and unit-automation. A full automation pipeline is the one where all steps of the process or assay are performed without any human intervention [85]. In unit automation, human intervention is required in certain 
stages and only individual stages in a pipeline are partially automated, independent of each other [86]. The advantage of unit-automation is that it keeps a highly open architecture of the pipeline allowing individual modules to be easily modified or exchanged; hence, the pipeline can be easily extended or modified [87].

Unlike the discovery of small molecule-based drugs in industry, where automation is a norm, development of novel aptamers has happened in individual labs in an academic research setting. For truly industrializing the aptamer discovery process, it is imperative to develop high-throughput strategies for aptamer development at different levels, viz., aptamer selection process, sequencing, and characterization of aptamers involving affinity and specificity studies toward its target. Here we discuss about the high-throughput technologies which have been developed facilitating high-throughput selection of aptamers.

The first attempt to automate aptamer selection process was performed by the group of Ellington [82] followed by some other improvements [88-92]. It brought down the selection time from weeks or months to days. Automation of the SELEX process increased the throughput of selections, but shifted the bottleneck within a selection pipeline toward the identification and evaluation side.

In 2011 the group of T.M. Soh developed a Quantitative Parallel Aptamer Selection System (QPASS), which integrates microfluidic aptamer selection (M-SELEX) and next-generation sequencing (NGS) with in situ synthesized aptamer arrays, enabling simultaneous measurement of affinity and specificity of several 1000 candidate aptamers in parallel. Major advantage of this high-throughput platform is that it automated the characterization, i.e., finding the affinity and specificity of aptamers which is a major bottleneck in aptamer selection, since traditionally the affinity and specificity of aptamers is measured individually in a serial manner [93]. This group used angiopoietin-2 (Ang2) as a target and performed four rounds of M-SELEX followed by next-generation sequencing to obtain enriched aptamer sequences. Next, using aptamer arrays they simultaneously measured dissociation constant $\left(K_{d}\right)$ of $\sim 1000$ aptamer candidates in parallel, identifying six high-affinity Ang2 aptamers with $\mathrm{Kd}<30 \mathrm{nM}$.

Development of NGS platform in the last decade has revolutionized the aptamer selection process. The Schroeder group in 2010 made the initial attempt in this direction as they combined genomic SELEX with high-throughput sequencing for the identification of genomic aptamers [94]. The same year, Soh group (2010) integrated microfluidic SELEX with high-throughput sequencing to obtain novel aptamers against platelet-derived growth factor BB (PDGF-BB) protein in just three rounds of selection, which traditionally takes 12-20 rounds [95]. Advantage of HTS is that it can track the copy number and enrichment fold of more than 10 million individual aptamer sequences through multiple rounds of selection. This enables the identification of high-affinity aptamers without the need of fully converging the aptamer pool to a small number of sequences, which is required for cloningbased selection method. Similarly, Hoon et al. obtained high-affinity aptamers after just one round of positive selection followed by HTS [96].

\section{Conclusion}

Since the inception of aptamer technology, this field has significantly evolved with its foray into a wide variety of applications, including targeted delivery. Aptamers caught the attention of researchers due to their unique advantages for use in therapeutics in nanomedicine due to their small size that gives higher penetration in tissues as compared to antibodies, ease of synthesis, high specificity and affinity that is comparable with antibodies and ease of chemical modifications that allowed 
easy conjugation with a variety of molecules like siRNA/miRNAs, proteins, peptides, antibody, drugs and nanoparticles. However, there have also been challenges in the application of aptamers due to their unique characteristics, viz., small size that lead to quick renal filtration, serum instability and lack of diversity in aptamer library. It is important to note that each of these issues has been addressed in different possible ways. Quick renal filtration of aptamers was tackled by conjugation with high molecular weight moieties, e.g., PEG. Similarly, serum instability was dealt with introduction of modifications to the nucleic acid backbone and modifications at $5^{\prime}$ - or $3^{\prime}$-end of the oligonucleotide. The diversity of aptamer library was increased by introduction of modified bases, and this lead to the development of mutant polymerases which were capable of amplifying these unnatural aptamers. Endosomal escape of cell-internalizing aptamers is a very important issue that needs to be addressed for clinical translation. Conjugation of aptamer with endosome escaping peptides will facilitate this issue. But lysosomal localization of aptamers is not always a problem, since lysosomal delivery of proteins or drugs is an important aspect of treating lysosomal storage diseases.

Efforts to up the speed of aptamer selection for industrializing this technology were achieved by development of high-throughput SELEX, and CE-SELEX which brought down the duration of selection process from months or weeks to just a few days. Additionally, the development of next-generation sequencing platform has been a boon for aptamer selection since it allowed assay of millions of aptamer sequences at one go, and alleviated the need to perform several rounds of selection that was earlier a norm. It significantly reduced the amount of time and resources that went in the selection of aptamers. From the point of selectionologists, it is quite important to characterize aptamers in the best possible way, i.e., keep right controls and perform vigorous validation experiments to make sure that aptamers coming out of a lab are robust, and work in hands of different people. Development of working reliable aptamers will pave their way for targeted delivery of therapeutics.

Macugen was the first FDA approved aptamer drug that appeared in market for clinical use in 2005. No other aptamer drug has entered the market since then. Several aptamers underwent clinical trials in the last 2 decades as listed in earlier reviews [5, 97, 98]. However, many of them have failed [5, 98]. In many cases, the failure in clinical trials is not due to lack of aptamer specificity or affinity to the target, but because they were unable to provide better treatment over the existing state-of-the-art treatment regimens. However, it is a common phenomenon that more drugs fail during clinical trails than pass it. In the past 2 decades the advances described in this chapter have facilitated in the development of stabilized aptamers that have entered clinical trials. In future, application of aptamers in targeted delivery and therapeutics is only poised to increase due to the establishment of ancillary technologies that facilitate faster and more stable aptamer development. Due to this, a number of aptamers are entering clinical trials. In summary, aptamermediated targeted delivery remains a work in progress; however, with bright results at in vivo level and several aptamer drugs in clinical trials, the coming decade will determine their efficiency at the clinical level.

\section{Acknowledgements}

We would like to thank Lakshmi Narayanan Gopalan for his assistance in the preparation of figures. The salary support for this work was provided by Indo-Finland research grant (IV4SP-FinSynBio-Synthetic Biology), funded by Department of Biotechnology, Government of India (Grant number BT/IN/ Finland/29/MN/2013). 


\section{Conflict of interest}

The authors declare no conflict of interest.

\section{Author details}

Chetan Chandola and Muniasamy Neerathilingam*

Department of Lipid Science, CSIR-Central Food Technological Research Institute, Mysore, India

*Address all correspondence to: mneerathilingam@gmail.com

\section{IntechOpen}

(C) 2019 The Author(s). Licensee IntechOpen. This chapter is distributed under the terms of the Creative Commons Attribution License (http://creativecommons.org/licenses/ by/3.0), which permits unrestricted use, distribution, and reproduction in any medium, provided the original work is properly cited. (cc) BY 


\section{References}

[1] Ellington AD, Szostak J. In vitro selection of RNA molecules that bind specific ligands. Nature. 1990;346:818-822

[2] Tuerk C, Gold L. Systematic evolution of ligands by exponential enrichment: RNA ligands to bacteriophage T4 DNA polymerase. Science. 1990;249:505-510

[3] Yoon S, Rossi JJ. Aptamers: Uptake mechanisms and intracellular applications. Advanced Drug Delivery Reviews. 2018;134:22-35. DOI: 10.1016/j. addr.2018.07.003

[4] Chandola C, Kalme S, Casteleijn MG, et al. Application of aptamers in diagnostics, drug-delivery and imaging. Journal of Biosciences. 2016;41:535-561

[5] Maier KE, Levy M. From selection hits to clinical leads: Progress in aptamer discovery. Molecular Therapy. Methods \& Clinical Development. 2016;3:16014

[6] Yang JP, Huang L. Time-dependent maturation of cationic liposome-DNA complex for serum resistance. Gene Therapy. 1998;5:380-387

[7] Crook K, Stevenson BJ, Dubouchet $\mathrm{M}$, et al. Inclusion of cholesterol in DOTAP transfection complexes increases the delivery of DNA to cells in vitro in the presence of serum. Gene Therapy. 1998;5:137-143

[8] Li S, Tseng WC, Beer Stolz D, et al. Dynamic changes in the characteristics of cationic lipidic vectors after exposure to mouse serum: Implications for intravenous lipofection. Gene Therapy. 1999;6:585-594

[9] Wheeler JJ, Palmer L, Ossanlou M, et al. Stabilized plasmid-lipid particles: Construction and characterization. Gene Therapy. 1999;6:271-281
[10] Raouane M, Desmaële D, Urbinati G, et al. Lipid conjugated oligonucleotides: A useful strategy for delivery. Bioconjugate Chemistry. 2012;23:1091-1104

[11] Hafez IM, Maurer N, Cullis PR. On the mechanism whereby cationic lipids promote intracellular delivery of polynucleic acids. Gene Therapy. 2001;8:1188-1196

[12] Xu Y, Szoka FC. Mechanism of DNA release from cationic liposome/DNA complexes used in cell transfection. Biochemistry. 1996;35:5616-5623

[13] Sakurai F, Nishioka T, Saito H, et al. Interaction between DNAcationic liposome complexes and erythrocytes is an important factor in systemic gene transfer via the intravenous route in mice: The role of the neutral helper lipid. Gene Therapy. 2001;8:677-686

[14] Lee CH, Lee SH, Kim JH, et al. Pharmacokinetics of a cholesterolconjugated aptamer against the hepatitis $\mathrm{C}$ virus (HCV) NS5B protein. Molecular Therapy. Nucleic Acids. 2015;4:e254

[15] Healy JM, Lewis SD, Kurz $\mathrm{M}$, et al. Pharmacokinetics and biodistribution of novel aptamer compositions. Pharmaceutical Research. 2004;21:2234-2246

[16] Yan X, Gao X, Zhang Z. Isolation and characterization of $2^{\prime}$-aminomodified RNA aptamers for human TNFalpha. Genomics, proteomics Bioinforma/Beijing Genomics Inst. 2004;2:32-42

[17] Kuwahara M, Sugimoto N. Molecular evolution of functional nucleic acids with chemical modifications. Molecules. 2010;15:5423-5444 
[18] Li N, Nguyen HH, Byrom M, et al. Inhibition of cell proliferation by an anti-egfr aptamer. PLoS One. 2011;6:1-10

[19] Derbyshire N, White SJ, Bunka DHJ, et al. Toggled RNA aptamers against aminoglycosides allowing facile detection of antibiotics using gold nanoparticle assays. Analytical Chemistry. 2012;84:6595-6602

[20] Green LS, Jellinek D, Bell C, et al. Nuclease-resistant nucleic acid ligands to vascular permeability factor VEGF. 1995;10:683-695

[21] Lebars I, Richard T, Di Primo C, et al. LNA derivatives of a kissing aptamer targeted to the trans-activating responsive RNA element of HIV-1. Blood Cells, Molecules \& Diseases. 2007;38:204-209

[22] Hernandez FJ, Stockdale KR, Huang L, et al. Degradation of nuclease-stabilized RNA oligonucleotides in mycoplasmacontaminated cell culture media. Nucleic Acid Therapeutics (Formerly Oligonucleotides). 2012;22:58-68 120109070352007

[23] Kuwahara M, Obika S. In vitro selection of BNA (LNA) aptamers. Artificial DNA PNA XNA. 2013;4:39-48

[24] Veedu RN, Wengel J. Locked nucleic acid nucleoside triphosphates and polymerases: On the way towards evolution of LNA aptamers. Molecular BioSystems. 2009;5:787-792

[25] Siddiqui MAA, Keating

GM. Pegaptanib: In exudative agerelated macular degeneration. Drugs. 2005;65:1571-1577

[26] Eaton BE. The joys of in vitro selection: Chemically dressing oligonucleotides to satiate protein targets. Current Opinion in Chemical Biology. 1997;1:10-16

[27] Nitsche A, Kurth A, Dunkhorst $A$, et al. One-step selection of vaccinia virus-binding DNA aptamers by MonoLEX. BMC Biotechnology. 2007;7:1-12

[28] Vater A, Klussmann S. Turning mirror-image oligonucleotides into drugs: The evolution of Spiegelmer ${ }^{\circledR}$ therapeutics. Drug Discovery Today. 2015;20:147-155

[29] Klussmann S, Nolte A, Bald R. Mirror-image RNA that binds D-adenosine. Nature Biotechnology. 1996;14:1112-1115

[30] Gold L, Ayers D, Bertino J, et al. Aptamer-based multiplexed proteomic technology for biomarker discovery. PLoS One. 2010;5:e15004. DOI: 10.1371/ journal.pone.0015004

[31] Kimoto M, Yamashige R, Matsunaga $\mathrm{K}$, et al. Generation of high-affinity DNA aptamers using an expanded genetic alphabet. Nature Biotechnology. 2013;31:453-457

[32] Sefah K, Yang Z, Bradley KM, et al. In vitro selection with artificial expanded genetic information systems. Proceedings of the National Academy of Sciences. 2014;111:1449-1454

[33] Temme JS, Krauss IJ. SELMA: Selection with modified aptamers. Current Protocols in Chemical Biology. 2015;7:73-92

[34] Zhou J, Rossi J. Aptamers as targeted therapeutics: Current potential and challenges. Nature Reviews. Drug Discovery. 2017;16:181-202

[35] Yang Q, Lai SK. Anti-PEG immunity: Emergence, characteristics, and unaddressed questions. 
Wiley Interdisciplinary Reviews. Nanomedicine and Nanobiotechnology. 2015;7:655-677

[36] Tucker CE, Chen LS, Judkins $\mathrm{MB}$, et al. Detection and plasma pharmacokinetics of an antivascular endothelial growth factor oligonucleotide-aptamer (NX1838) in rhesus monkeys. Journal of Chromatography. B, Biomedical Sciences and Applications. 1999;732:203-212

[37] Drolet DW, Nelson J, Tucker CE, Zack PM, Nixon K, Bolin R, et al. Pharmacokinetics and safety of an anti-vascular endothelial growth factor aptamer (NX1838) following injection into the vitreous humor of rhesus monkeys Daniel. Pharmaceutical Research. 2000;17:24-31

[38] Da Pieve C, Blackshaw E, Missailidis $\mathrm{S}$, et al. PEGylation and biodistribution of an anti-MUC1 aptamer in MCF-7 tumor-bearing mice. Bioconjugate Chemistry. 2012;23:1377-1381

[39] Prodeus A, Abdul-Wahid A, Fischer NW, et al. Targeting the PD-1/ PD-L1 immune evasion axis with DNA aptamers as a novel therapeutic strategy for the treatment of disseminated cancers. Molecular Therapy. Nucleic Acids. 2015;4:e237

[40] Willis MC, Collins B, Zhang $\mathrm{T}$, et al. Liposome-anchored vascular endothelial growth factor aptamers. Bioconjugate Chemistry. 1998;9:573-582

[41] Guyer DR, Fish G, Haller JA, et al. Anti-vascular endothelial growth factor therapy for subfoveal choroidal neovascularization secondary to age-related macular degeneration: Phase II study results. Ophthalmology. 2003;110:979-986

[42] Martin DF, Klein M, Haller J, et al. Preclinical and phase $1 \mathrm{~A}$ clinical evaluation of an anti-VEGF pegylated aptamer (EYE001) for the treatment of exudative age-related macular degeneration. Retina. 2002;22:143-152

[43] Gragoudas ES, Adamis AP, Cunningham ET, et al. Pegaptanib for neovascular age-related macular degeneration. The New England Journal of Medicine. 2004;351:2805-2816

[44] De Campos WRL, Coopusamy D, Morris L, et al. Cytotoxicological analysis of a gp120 binding aptamer with cross-clade human immunodeficiency virus type 1 entry inhibition properties: Comparison to conventional antiretrovirals. Antimicrobial Agents and Chemotherapy. 2009;53:3056-3064

[45] Zheng J, Zhao S, Yu X, et al. Simultaneous targeting of CD44 and EpCAM with a bispecific aptamer effectively inhibits intraperitoneal ovarian cancer growth. Theranostics. 2017;7:1373-1388

[46] Swayze EE, Siwkowski AM, Wancewicz EV, et al. Antisense oligonucleotides containing locked nucleic acid improve potency but cause significant hepatotoxicity in animals. Nucleic Acids Research. 2007;35:687-700

[47] Lee Y, Urban JH, Xu L, et al. 2'Fluoro modification differentially modulates the ability of RNAs to activate pattern recognition receptors. Nucleic Acid Therapeutics. 2016;26:173-182

[48] Farman CA, Kornbrust DJ. Oligodeoxynucleotide studies in primates: Antisense and immune stimulatory indications. Toxicologic Pathology. 2003;31:119-122

[49] Stark AM, Pfannenschmidt S, Tscheslog H, et al. Reduced mRNA and protein expression of BCL-2 versus decreased mRNA and increased 
protein expression of BAX in breast cancer brain metastases: A real-time PCR and immunohistochemical evaluation. 2006;28:787-793. DOI: $10.1179 / 016164106 \mathrm{X} 110364$

[50] Marquis JK, Grindel

JM. Toxicological evaluation of oligonucleotide therapeutics. Current Opinion in Molecular Therapeutics. 2000;2:258-263

[51] Sundaram P, Kurniawan $H$, Byrne ME, et al. Therapeutic RNA aptamers in clinical trials. European Journal of Pharmaceutical Sciences. 2013;48:259-271

[52] Bouchard PR. Discovery and development of therapeutic aptamers. Annual Review of Pharmacology and Toxicology. 2010;50:237-257

[53] Weiner GJ, Liu H-M, Wooldridge $\mathrm{JE}$, et al. Immunostimulatory oligodeoxynucleotides containing the CpG motif are effective as immune adjuvants in tumor antigen immunization. Proceedings of the National Academy of Sciences. 1997;94:10833-10837

[54] Bauer S, Wagner H. Bacterial CpGDNA licenses TLR9. Current Topics in Microbiology and Immunology. 2002;270:145-154

[55] Rothenfusser S, Tuma E, Endres S, et al. Plasmacytoid dendritic cells: The key to CpG. Human Immunology. 2002;63:1111-1119

[56] Fey R, Zanardi T, Kramer-Stickland $\mathrm{K}$, et al. Toxicological properties of 2'-O-methoxyethyl chimeric antisense inhibitors in animals and man. In: Antisense Drug Technology: Principles, Strategies, and Applications. 2nd ed. 2007. p.327-363. DOI: 10.1201/9780849387951.ch12

[57] Guo S, Li H, Ma M, et al. Size, shape, and sequence-dependent immunogenicity of RNA nanoparticles. Molecular Therapy. Nucleic Acids. 2017;9:399-408

[58] Ganson NJ, Povsic TJ, Sullenger BA, et al. Pre-existing anti-polyethylene glycol antibody linked to first-exposure allergic reactions to pegnivacogin, a PEGylated RNA aptamer. Journal of Allergy and Clinical Immunology. 2016;137:1610-1613e7

[59] Dou X, Wang H, Zhang J, Wang F, et al. Aptamer-drug conjugate: Targeted delivery of doxorubicin in a HER3 aptamer-functionalized liposomal delivery system reduces cardiotoxicity. International Journal of Nanomedicine. 2018;13:763-776

[60] Dam DHM, Culver KSB, Kandela I, Lee RC, et al. Biodistribution and in vivo toxicity of aptamer-loaded gold nanostars. Nanomedicine. 2015;11:671-679

[61] Lai WY, Huang BT, Wang JW, Lin PY, et al. A novel PD-L1-targeting antagonistic DNA aptamer with antitumor effects. Molecular Therapy. Nucleic Acids. 2016;13:e397

[62] Vinores SA. Pegaptanib in the treatment of wet, age-related macular degeneration. International Journal of Nanomedicine. 2006;1:263-268

[63] Sørensen HP, Mortensen KK. Soluble expression of recombinant proteins in the cytoplasm of Escherichia coli. Microbial Cell Factories. 2005;4:1-8

[64] Liu Y, Kuan CT, Mi J, et al. Aptamers selected against the unglycosylated EGFRvIII ectodomain and delivered intracellularly reduce membrane-bound EGFRvIII and induce apoptosis. Biological Chemistry. 2009;390:137-144

[65] Mercier MC, Dontenwill M, Choulier L. Selection of nucleic acid aptamers targeting tumor 
cell-surface protein biomarkers. Cancers (Basel). 2017;9(6):69. DOI: 10.3390/ cancers 9060069

[66] Thiel KW, Hernandez LI, Dassie JP, et al. Delivery of chemo-sensitizing siRNAs to HER2+-breast cancer cells using RNA aptamers. Nucleic Acids Research. 2012;40:6319-6337

[67] Wilner SE, Wengerter B, Maier K, et al. An RNA alternative to human transferrin: A new tool for targeting human cells. Molecular Therapy. Nucleic Acids. 2012;1:e21

[68] Magalhães MLB, Byrom M, Yan A, et al. A general RNA motif for cellular transfection. Molecular Therapy.

2012;20:616-624

[69] Iaboni M, Fontanella R, Rienzo A, et al. Targeting insulin receptor with a novel internalizing aptamer. Molecular Therapy. Nucleic Acids. 2016;5:e365

[70] Dowdy SF. Overcoming cellular barriers for RNA therapeutics. Nature Biotechnology. 2017;35:222-229

[71] Li M, Tao Y, Shu Y, et al. Discovery and characterization of a peptide that enhances endosomal escape of delivered proteins in vitro and in vivo. Journal of the American Chemical Society. 2015;137:14084-14093

[72] Lönn P, Kacsinta AD, Cui XS, et al. Enhancing endosomal escape for intracellular delivery of macromolecular biologic therapeutics. Scientific Reports. 2016;6:1-9

[73] Salomone F, Cardarelli F, Di Luca M, et al. A novel chimeric cellpenetrating peptide with membranedisruptive properties for efficient endosomal escape. Journal of Controlled Release. 2012;163:293-303

[74] Bareford LM, Swaan PW. Endocytic mechanisms for targeted drug delivery. Advanced Drug Delivery Reviews. 2007;59:748-758
[75] Wagenaar TR, Tolstykh T, Shi C, et al. Identification of the endosomal sorting complex required for transport-I (ESCRT-I) as an important modulator of anti-miR uptake by cancer cells. Nucleic Acids Research. 2015;43:1204-1215

[76] Wang H, Tam YYC, Chen S, et al. The niemann-pick $\mathrm{C} 1$ inhibitor NP3.47 enhances gene silencing potency of lipid nanoparticles containing siRNA. Molecular Therapy. 2016;24:2100-2108

[77] Behr JP. The proton sponge: A trick to enter cells the viruses did not exploit. Chim. International Journal of Chemistry. 1997;51:34-36

[78] Liu HA, Gao X. A universal protein tag for delivery of siRNAaptamer chimeras. Scientific Reports. 2013;3:3129-3136

[79] Bates PJ, Kahlon JB, Thomas SD, et al. Antiproliferative activity of G-rich oligonucleotides correlates with protein binding. The Journal of Biological Chemistry. 1999;274:26369-26377

[80] Tawiah K, Porciani D, Burke D. Toward the selection of cell targeting aptamers with extended biological functionalities to facilitate endosomal escape of cargoes. Biomedicine. 2017;5:51

[81] Yan AC, Levy M. Aptamer-mediated delivery and cell-targeting aptamers: Room for improvement. Nucleic Acid Therapeutics. 2018;28:194-199

[82] Cox JC, Rudolph P, Ellington AD. Automated RNA selection. Biotechnology Progress. 1998;14:845-850

[83] Bairy S, Gopalan LN, Setty G, et al. Automation aided optimization of cloning, expression and purification of enzymes of the bacterial sialic acid catabolic and sialylation pathways enzymes for structural 
studies. 2018;11:420-428. DOI: 10.1111/1751-7915.13041

[84] Hamilton S. Introduction to screening automation. High-Throughput Screening. 2002;190:169-189

[85] Cohen S, Trinka RF. Fully automated screening systems. Methods in Molecular Biology. 2002;190:213-228

[86] Menke KC. Unit automation in high throughput screening. Methods in Molecular Biology. 2002;190:195-212

[87] Konthur Z. Automation of selection and engineering. In: Handbook of Therapeutic Antibodies. Weinhem: Wiley-VCH; 2007. pp. 413-431

[88] Cox JC, Ellington AD. Automated selection of anti-protein aptamers. Bioorganic \& Medicinal Chemistry. 2001;9:2525-2531

[89] Cox JC, Hayhurst A, Hesselberth J, et al. Automated selection of aptamers against protein targets translated in vitro: From gene to aptamer. Nucleic Acids Research. 2002;30:e108

[90] Cox JC, Rajendran M, Riedel T, et al. Automated acquisition of aptamer sequences. Combinatorial Chemistry \& High Throughput Screening. 2002;5:289-299

[91] Drolet DW, Jenison RD, Smith DE, et al. A high throughput platform for systematic evolution of ligands by exponential enrichment (SELEX). Combinatorial Chemistry \& High Throughput Screening. 1999;2:271-278

[92] Eulberg D, Buchner K, Maasch C, et al. Development of an automated in vitro selection protocol to obtain RNA-based aptamers: Identification of a biostable substance P antagonist. Nucleic Acids Research. 2005;33:1-10

[93] Cho M, Soo Oh S, Nie J, et al. Quantitative selection and parallel characterization of aptamers.

Proceedings of the National Academy of Sciences. 2013;110:18460-18465

[94] Zimmermann B, Gesell T, Chen D, et al. Monitoring genomic sequences during SELEX using high-throughput sequencing: Neutral SELEX. PLoS One. 2010;5:1-7

[95] Cho M, Xiao Y, Nie J, et al.

Quantitative selection of DNA aptamers through microfluidic selection and highthroughput sequencing. Proceedings of the National Academy of Sciences. 2010;107:15373-15378

[96] Hoon S, Zhou B, Janda KD, et al. Aptamer selection by high-throughput sequencing and informatic analysis. BioTechniques. 2011;51:413-416

[97] Kaur H, Bruno JG, Kumar A, Sharma TK. Aptamers in the therapeutics and diagnostics pipelines. Theranostics. 2018;8:4016-4032

[98] Kanwar JR, Roy K, Maremanda NG, Subramanian K, et al. Nucleic acid-based aptamers: Applications, development and clinical trials. Current Medicinal Chemistry. 2015;22:2539-2557 


\title{
Breaking down the Barrier: Topical Liposomes as Nanocarriers for Drug Delivery into the Posterior Segment of the Eyeball
}

\author{
Arturo Santos, Juan C. Altamirano-Vallejo, \\ José Navarro-Partida, Alejandro González-De la Rosa \\ and Jane H. Hsiao
}

\begin{abstract}
Topical instillation is the most widely preferred noninvasive route of drug administration to treat diseases affecting the anterior segment of the eye. Nonetheless, the ocular bioavailability for deeper ocular tissues is very low. Different routes of administration, such as intravitreal injections, periocular injections, and systemic administration, have been used to deliver drugs into the posterior segment ocular tissues. However, the presence of blood-retinal barriers (BRBs) makes systemic administration an impractical approach, whereas the drug delivery with the periocular administration route is compromised by ocular static and dynamic barriers. On the other hand, intravitreal injection, the most common and widely recommended route for drug administration to treat posterior ocular diseases, is related to several side effects such as endophthalmitis, hemorrhage, retinal detachment, and poor patient tolerance. Diverse strategies to overcome ocular barriers have been explored for topical drop formulations in order to deliver drugs into the posterior segment ocular tissues. In this chapter, we will review the promising topical nanocarriers for drug delivery into the posterior segment of the eye, emphasizing the use of liposomes for topical ophthalmic formulations targeting the vitreous cavity and the retina.
\end{abstract}

Keywords: liposomes, retina, vitreous, ocular posterior segment, nanocarriers, novel drug delivery system

\section{Introduction and objective}

The eyeball may be divided into two parts: the anterior and the posterior segments. The anterior segment consists of the pupil, cornea, iris, ciliary body, aqueous humor, and lens, whereas the posterior segment is comprised of the vitreous humor, macula, retina, choroid, and optic nerve. The retina is the sensitive layer which creates nerve impulses that are transmitted through the optic nerve to the brain. The macula is a specialized area of the retina that is responsible for the central, high-resolution color vision [1]. The retina and macula can suffer from 
different disabling illnesses, such as age-related macular degeneration, cystoid macular edema, diabetic retinopathy, and ocular vascular occlusion, which are the leading causes of nonreversible vision impairment $[2,3]$. These retinal disorders could be pharmacologically addressed; however, the efficient and safe delivery of drugs to the retinal tissue is not a completely solved subject.

Compared to drug delivery to other organs, ocular drug delivery faces significant challenges posed by various sophisticated ocular barriers. Many of these barriers are inherent and unique to ocular anatomy and physiology, making it a challenging task for drug delivery innovations. Corneal and conjunctival epithelium, bloodaqueous barriers (BAB), and blood-retinal barriers (BRBs) are the fundamental structures that restrict the passage of molecules and fluids to the retina and impede drug penetration; moreover, various elimination mechanisms, such as tear turnover, nasolacrimal drainage, protein binding, systemic absorption, and enzymatic degradation, limit the ocular bioavailability of drugs [4, 5]. Conventional drug administration systems such as eye drops, suspensions, and ointments are optimal in the treatment of the ocular surface (corneal and conjunctiva tissues) and anterior segment disorders. Nevertheless, due to the ocular barriers, drugs barely get into the posterior ocular segment [1]. It is well known that ocular bioavailability after the topical administration of a drug is generally $<5 \%[4,5]$.

Therefore, intravitreal (IVT) injections, which circumvent the ocular barriers, are the most frequent pathway to deliver drugs for the treatment of posterior ocular globe disorders. Today, the use of IVT injections has become the most common intraocular procedure worldwide [6] and the standard drug delivery method for the treatment of retinal diseases [2, 3]. Intravitreal injections are now routinely used for the intraocular administration of drugs such as corticosteroids, antimetabolites, antibiotics, and anti-VEGF therapies [7-11].

Although IVT injections are a well-described and feasible route for releasing drugs into the posterior pole of the eye, this procedure is associated with severe complications such as endophthalmitis, lens injury, and retinal detachment [12-14]. Moreover, it might be a burden for physicians, the health system, and patients with poor compliance in many cases [15]. Furthermore, the administration of IVT injections requires highly specialized human resources and special infrastructure, resulting in an expensive therapy option [16]. Research initiatives are continuously being proposed worldwide at fast pace by apex organizations and pharmaceutical companies in order to find a safer and more effective ocular drug delivery method for ocular use.

Nanostructured carriers or nanocarriers (nanomaterials) have proven to be an effective and slightly invasive drug delivery system to keep drug concentrations in the posterior segment of the eyeball, preventing the use of IVT injections or reducing their frequency. The advantage of using nanocarriers is their ability to increase the biopharmaceutical properties of the incorporated drug: solubility, stability, permeability, and retention at the site of application [17].

Nanocarriers are made of nanoparticles (NPs) (1-1000 nm), and they exemplify one of the multiple strategies of nanomedicine, which is interpreted as the application of NPs for medical purposes [18]. The most commonly used materials for NPs include lipids (liposomes), proteins (albumin NPs), cyclic oligosaccharides (cyclodextrins), synthetic polymers (polymeric micelles, dendrimers, hydrogel), and even inorganic compounds (cerium oxide NPs) [19]. Liposomes have been of much interest as carriers for advanced drug delivery in medicine and, especially, in ophthalmology for their potential to avoid the sophisticated ocular barriers, even when they are topically applied. In fact, liposomes and cyclodextrins are the only topically administered nanoparticles that have successfully proven to release corticosteroids in clinical assays [20, 21].

In the following sections, we will review the ocular barriers and routes for ocular drug delivery and discuss the different nanocarriers topically used in preclinical 
studies that have proven to elude the anatomic and physiologic conditions that prevent drugs from reaching the tissues in the posterior segment of the eyeball, emphasizing the leading role of liposomes.

\section{Barriers for ocular drug delivery}

The different barriers that hinder intraocular drug delivery may be classified into three categories (Table 1).

\subsection{Static barriers}

The static barriers in the eye are physical barriers that prevent the diffusion of drug molecules into the posterior segment of the eye and the retina. The cornea is a mechanical barrier that comprises the anterior sixth of the eye. This transparent, ellipsoid barrier has different layers that play an important role in drug permeation: each layer offers a different polarity and a potential rate-limiting structure for drug permeation. The corneal epithelium is lipoidal in nature, and it contains $90 \%$ of cells in the cornea. These cells are joined by desmosomes and surrounded by ribbonlike tight junctional complexes. The presence of the zonula occludens delays paracellular drug permeation from the tear film into intracellular spaces of the epithelium, as well as into the inner layers of the cornea [22]. The epithelium has a significant resistance against the permeation of topical hydrophilic drugs. The highly hydrated stroma that comprises $90 \%$ of the corneal layer is made up by an extracellular matrix and a lamellar arrangement of collagen fibrils, and it is an important barrier against the permeation of lipophilic drug molecules. The hexagonal-shaped cells, or endothelium, represent the innermost layer of the cornea, and they have a selective

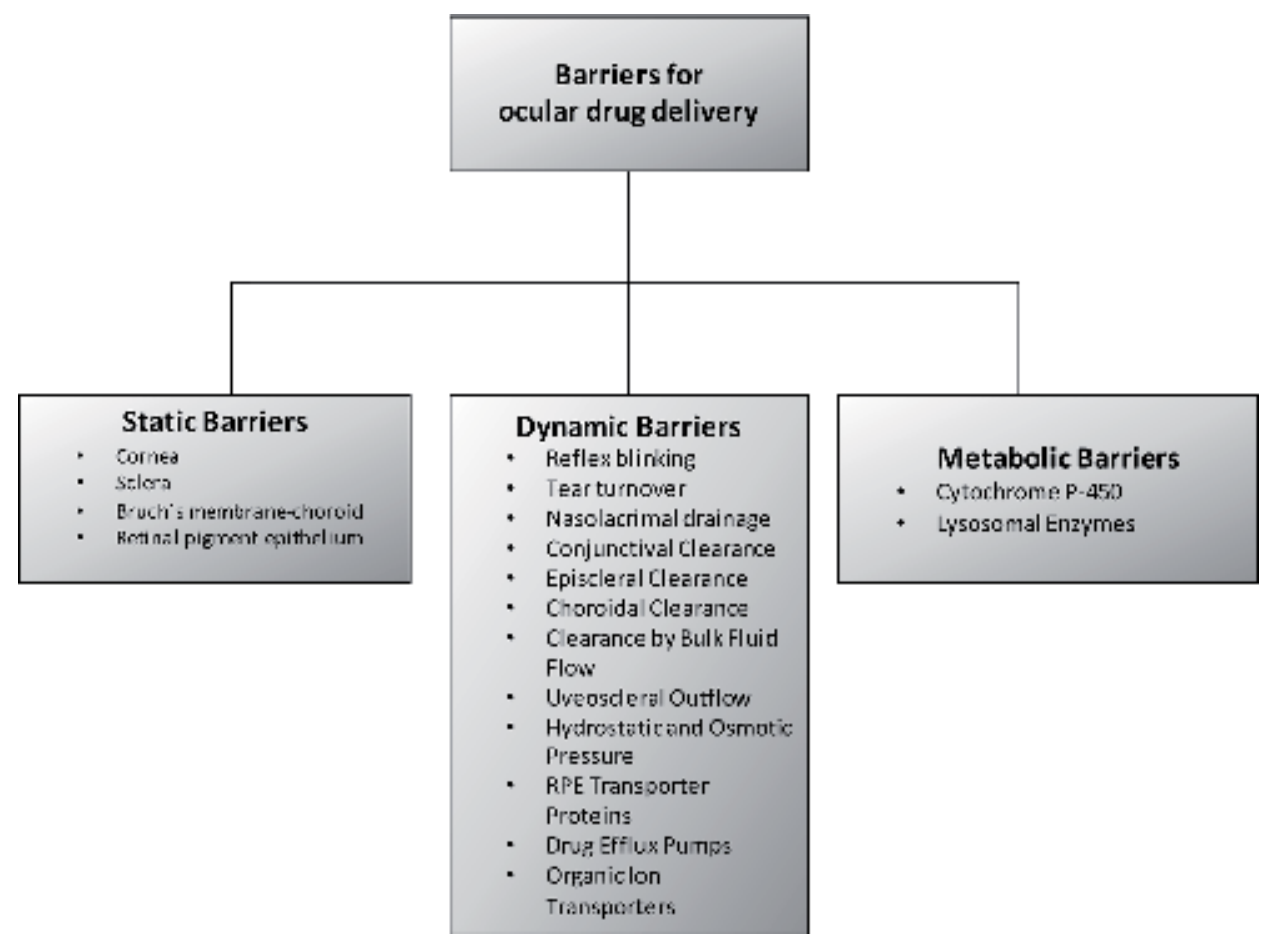

Table 1.

Types of barriers for ocular drug delivery. 
carrier-mediated transport and a secretory function. New drug delivery systems should have an amphipathic nature in order to permeate through the cornea [23].

The sclera forms the firm, fibrous, outermost layer of the eye. It keeps the shape of the eye and provides an attachment for the insertion of the extraocular muscles. It is about $1 \mathrm{~mm}$ thick at the site where the optic nerve pierces it. The sclera is made of collagen and elastin chains that create a fiber matrix, where the pore diameter and intracellular spaces may determine the flow of drugs. Furthermore, the lateral orientation of fibers, the differences in the collagen architecture of the posterior sclera, and the differences in myopic eyes may affect drug transport with a lower or higher permeability depending on these changes. The permeability of the sclera for a number of molecules, such as dextrans, polyethylene glycol, anti-angiogenic molecules, antibiotics, oligonucleotides, and lipophilic compounds, has been measured [24-30]. There are different factors that may affect the scleral permeability of these molecules [2, 31-33]. Scleral permeability has a strong dependence on molecular weight, with smaller molecules having a better permeability. Similarly, since globular proteins are more permeable than linear dextrans of the same molecular weight, molecular radius is an important predictor of scleral permeability [32]. Finally, any surgical, pathological, or traumatic change in the anatomy of the sclera may lead to permeability changes [34].

Different studies on Bruch's membrane - the choroid and the retinal pigment epithelium (RPE) — show that permeability increases when lipophilicity does the same. Molecules that are passively transported across the RPE show similar permeability values in both outward (retina-choroid) and inward (choroid-retina) directions, while molecules that are actively transported show differences in permeability between the two of them [22]. The presence of RPE melanin may alter ocular drug disposition. Melanin binds to free radicals and drugs through electrostatic and van der Waals forces or through simple charge-transfer interactions with this pigment, which may alter the availability of the free drug at the targeted site [35]. All basic and lipophilic drugs bind to melanin; thereby, melanin binding may significantly lower pharmacological activity [36]. As a result of the presence of melanin, the binding of lipophilic compounds to the choroid-Bruch's membrane is higher; consequently, there is greater resistance to solute permeation across the choroidBruch's membrane than across the sclera, which is devoid of melanin [37-39].

Aging does not alter the permeability or ultrastructure of the sclera, but the permeability of Bruch's membrane and the choroid has shown to be significantly affected by age $[2,34]$. Bruch's membrane may be a major resistance barrier against the movement of small solutes due to an increase in its thickness with aging (from $2 \mu \mathrm{m}$ in the first decade of life to $4.7 \mu \mathrm{m}$ in the tenth decade). Moreover, the accumulation of lipid-rich membranous debris and basal laminar deposits may have an important role in drug delivery [39].

\subsection{Dynamic barriers}

Dynamic barriers include the clearance through lymphatic and blood vessels, bulk fluid flow, and the active transport mechanisms of RPE transporter proteins. Precorneal barriers are highly effective and include solution drainage, blinking, tear film, tear turnover, and induced lacrimation.

Blinking is a normal reflex that protects the eye from dryness, bright light, and fingers or other objects coming toward it. Blinking also regulates tears, which nourish and cleanse the surface of the eye. The blinking rate in newborns is only two times per minute. This increases to 14-17 times per minute in adolescence and remains at this rate throughout the rest of the lifetime. Blinking may also increase in response to pain, bright light, changes in temperature and humidity, and conversations. 
The tear film offers resistance, thanks to its high turnover rate. Mucin plays a protective role in tear film by forming a hydrophilic layer that moves over to the glycocalyx of the ocular surface and clears debris and pathogens $[29,40]$. Human tear volume is estimated to be 7-10 $\mu \mathrm{l}$, and the cul-de-sac can transiently contain around $30 \mu \mathrm{l}$ of the administered eye drop. However, tear film displays a rapid restoration period of 2-3 min, and most of the topically administered solutions are washed away within just 15-30 s after instillation. Considering all of the precorneal factors, contact time with the absorptive membranes is lower, which is considered to be the primary reason for less than $5 \%$ of the applied dose reaching the intraocular tissues [41]. Precorneal fluid drainage is one of the main reasons for low ocular drug absorption [42-45].

After instillation, a big portion of an instilled volume (approximately from 80 to $90 \%$ ) is drained into the nasolacrimal duct. Nasolacrimal drainage helps maintain the volume of precorneal fluid at about 7-10 $\mu \mathrm{l}$ at all times [46]. A natural protective physiological mechanism causes the loss of any excess fluid present: it is drained out through the nasolacrimal duct. Similarly, other factors such as the instilled volume, viscosity, $\mathrm{pH}$, tonicity, and drug type may also alter the regular ocular physiological process. The higher the instilled volume, the higher the rate of solution drainage; increasing the drug's viscosity may extend contact time. Excessive tear production is associated with the instillation of acidic or alkaline solutions; hence the ideal ophthalmic formulation for topical delivery should be isotonic with tear secretions.

The conjunctiva is another effective barrier against ocular drug delivery. It is well vascularized, and drug molecules present in the conjunctiva and episcleral tissues are cleared through blood and/or lymphatic vessels $[47,48]$. Subconjunctively injected tracers have been detected in the cervical lymph nodes within 6 min [49, 50]. Furthermore, molecular size and molecular radius may affect the rate of clearance [2]. Future drug delivery systems will have to consider conjunctival/episcleral clearance mechanisms, given that they play a significant role in reducing intraocular drug penetration.

The choroid is a dark brown, highly vascularized layer located between the sclera and the retina. It extends from the ora serrata to the aperture of the optic nerve in the sclera. Drug molecules that are topically, systemically, and orally administered may be eliminated by the uptake of the rapid blood flow of the choroid. Drugs can be carried away by bulk fluid flows in ocular tissues and are ultimately mostly cleared through choroidal vasculature or conjunctival vascular and/or lymphatic vessels. Uveoscleral drainage has been reported within a range of 4-60\% [51], and it generates an outward bulk flow fluid from the suprachoroidal space. Many drugs may be carried away by the convective current of aqueous humor and cleared by the conjunctival vascular and/or lymphatic vessels. The effect of aging on uveoscleral outflow must also be considered by future drug delivery systems.

The osmotic pressure of the choroid in rabbits is $12-14 \mathrm{mmHg}$, and the vitreous humor has an osmotic pressure of approximately $0-1 \mathrm{mmHg}$ [52]. This difference in osmotic pressure between the choroid and the vitreous generates a fluid flow toward the choroid. The hydrostatic pressure difference between the suprachoroid and the episcleral tissue also contributes to the outward bulk fluid flow [53]. There is a hydrostatic pressure difference of about $12 \mathrm{mmHg}$ between the suprachoroid and the episcleral tissue, which works as a driving force for the outward bulk fluid flow. Drug efflux pumps, such as P-glycoproteins (P-gp) and multidrug resistanceassociated proteins (MRPs), have been reported in RPE with an efflux effect directed toward the choroid $[54,55]$. Finally, organic ion transporters may have an 
important role as active transport for drugs depending on extracellular $\mathrm{pH}$, temperature, and drug concentration [56].

\subsection{Metabolic barriers}

Transporters are membrane-bound proteins that play an important role in the active transport of nutrients or xenobiotics. These transporters play a significant role in humans in the processes of drug absorption, distribution, metabolism, and elimination. The presence of both efflux and influx transporters has been reported in various ocular tissues. The presence of efflux pumps in various ocular tissues has shown to regulate the intracellular drug concentration needed to achieve therapeutic activity. Drug-metabolizing enzymes are present in many ocular tissues such as the ciliary body and RPE [57, 58]. As a defense mechanism, the eye possesses several sophisticated metabolizing enzyme systems that degrade drug molecules, including those of the cytochrome P-450 (CYP) family, which are the most important drugmetabolizing enzymes. CYP3A isozyme, for example, metabolizes over $50 \%$ of commercial drugs [59].

\section{Routes for drug delivery into the posterior segment of the eye}

Designing a drug delivery system to target a specific tissue inside the eye is a challenge due to the different ocular barriers. Different modes of administration for drugs are available for ophthalmologists, but many of them fail to reach a therapeutic dose of the drug to elicit clinical effects. The different routes of ocular drug administration (Figure 1) include topical, oral/systemic, subconjunctival, subtenon, retrobulbar, intracameral, and intravitreal injections.

\subsection{Topical administration}

Topical administration is the most common method for drug administration in ophthalmology, mostly in the form of eye drops due to the ease of administration and the low cost. Topical application is useful in the treatment of disorders affecting the anterior segment of the eye [60]. The site of contact is the cornea, the conjunctiva, and the sclera. A normal eyedropper delivers from 25 to $56 \mu \mathrm{L}$ of topical formulation with an average volume of $39 \mu \mathrm{L}$. However, an eye can transiently hold up to $30 \mu \mathrm{L}$, and the rest is lost either through nasolacrimal drainage or reflex blinking (5-7 blinks/min), significantly decreasing the overall drug available for therapeutic action. Furthermore, after a drop is administered to the eye, a major fraction of the drug is lost through lacrimation, tear dilution, nasolacrimal drainage, and tear turnover following topical administration. Such precorneal losses result in very low ocular bioavailability. Typically, less than $5 \%$ of the total administered dose reaches the aqueous humor $[22,61]$. Therefore, in order to maintain minimum inhibitory concentrations, the agents need to be frequently dosed, resulting in poor patient compliance or potential adverse events. Physical barriers such as the cornea and the sclera and dynamic barriers such as reflex blinking, tear turnover, nasolacrimal drainage, conjunctival clearance, episcleral clearance, choroidal clearance, and clearance through bulk fluid flow and uveoscleral outflow are some of the many sophisticated barriers that cause poor drug molecule concentrations in the target organ (retina). For these reasons, topical administration is regarded as a poor option for the treatment of posterior diseases such as macular edema, age-related macular degeneration, and diabetic retinopathy. 


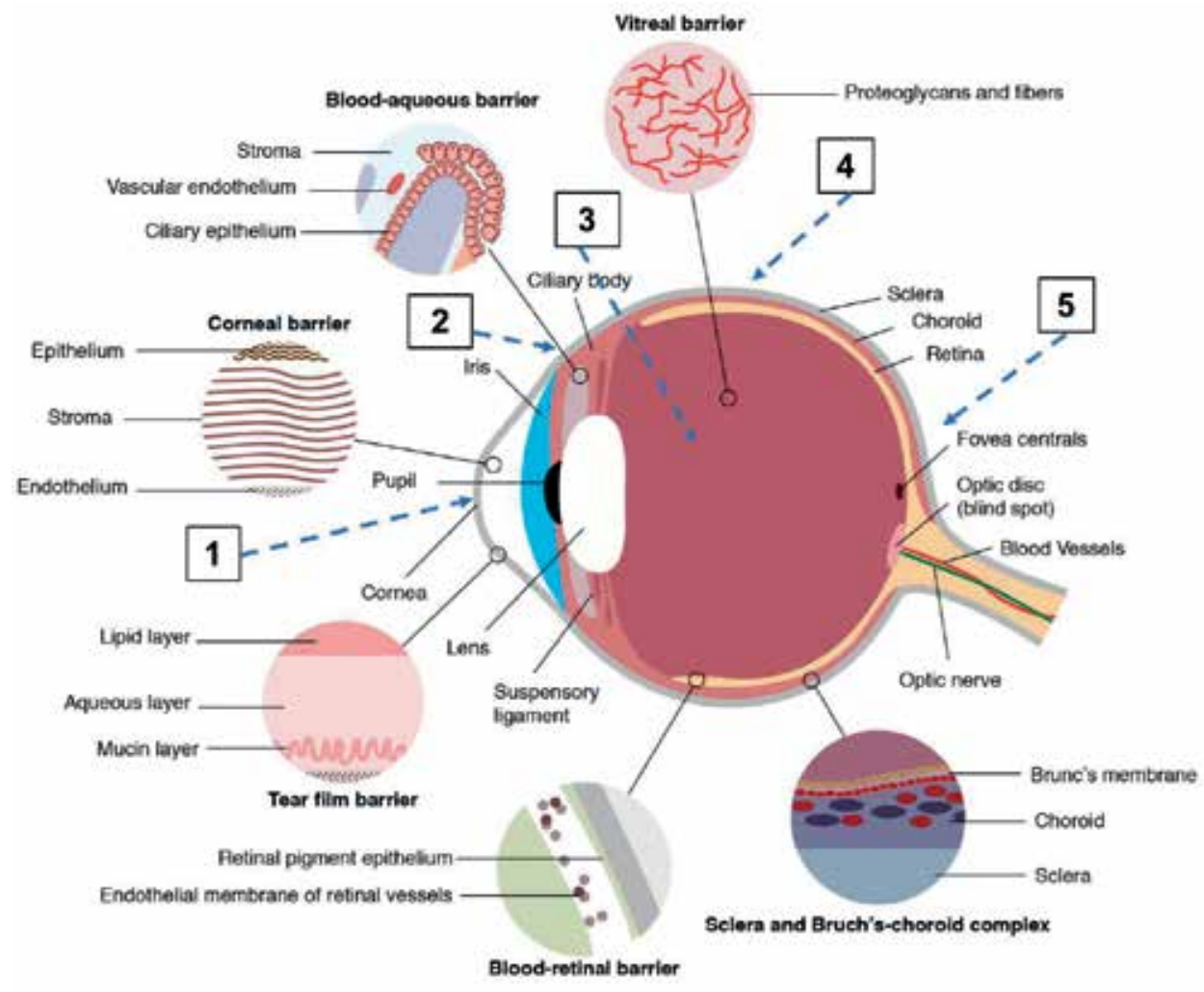

Figure 1.

Barriers for ocular drug delivery and different routes of drug administration to the eye. (1) Topical; (2) subconjunctival/subtenon; (3) intravitreal; (4) peribulbar; and (5) retrobulbar.

\subsection{Oral/systemic administration}

The oral and systemic administration of drugs is the most common drug delivery form in medicine (but this is not the case for eyes). Following oral/systemic administration, the blood-aqueous and blood-retinal barriers are major problems for drug delivery. The ciliary epithelium produces aqueous humor, which acts as an ultrafilter and restricts the entry of macromolecules such as antibiotics and plasma proteins [42]. Drug delivery to the posterior pole and the retina is very difficult due to the fact that blood-retinal barriers prevent the diffusion of drugs into the posterior pole of the eye. BRBs are composed of retinal capillary endothelial cells and RPE, which are known as the inner and outer blood-retinal barriers, respectively. RPE is a monolayer of highly specialized cells, and it aids in biochemical functions by selectively transporting molecules between photoreceptors and choriocapillaris [38]. However, the tight junctions of RPE efficiently restrict intercellular permeation.

Drugs can easily enter the choroid, thanks to its high vasculature compared to that of retinal capillaries. The choriocapillaris are fenestrated, resulting in rapid equilibration of drug molecules in the bloodstream with the extravascular space of the choroid. However, RPE restricts further entry of drugs from the choroid to the retina. Because the blood-retinal barrier, which is selectively permeable to more lipophilic molecules, mainly governs the entry of drug molecules into the posterior segment of the eye, this results in the need for frequent administration of high amounts of drugs, which leads to systemic side effects [62]. 
Although topical and systemic routes are convenient, the lack of adequate bioavailability and the failure to deliver therapeutic amounts of drugs into the retina prompted vision scientists to search for alternative routes of administration. Hence, specific oral or intravenous targeting systems are needed to transport molecules through the choroid and into deeper layers of the retina [43]. An example of a systemic drug for the treatment of retinal diseases is Visudyne, which is used in photodynamic therapy for the treatment of wet age-related macular degeneration (AMD) and central serous chorioretinopathy (CSC). However, owing to toxicity and delivery concerns, intravenous administration is not very common in treating ocular diseases.

\subsection{Periocular administration}

Although not very patient compliant, these routes are partly used to overcome the inefficiency of topical and systemic dosing in order to deliver therapeutic drug concentrations to the posterior segment of the eye globe. Moreover, systemic administration may lead to side effects, making it a less desirable delivery route for geriatric patients. Periocular routes include subconjunctival, subtenon, retrobulbar, and peribulbar administrations, and they are comparatively less invasive than intravitreal routes. Drugs administered by periocular injections can reach the posterior segment through three different pathways: the transscleral pathway, systemic circulation through the choroid, and the anterior pathway through the tear film, cornea, aqueous humor, and vitreous humor [63]. The administration of a drug via subtenon injection resulted in the highest and more sustained vitreous concentration of drug molecules compared to other periocular injections [64]. However, anterior segment complications have been described in some patients following periocular injections. The complications include ocular hypertension, cataract, hyphema, strabismus, and corneal decompensation [65]. Additionally, the concentration of many drugs, such as that of anti-angiogenic drugs, is better if an intravitreal injection is used as a delivery pathway.

\subsection{Intravitreal injection}

Nowadays, intravitreal injection is the preferred route for ocular delivery for the treatment of diseases of the retina. It offers important advantages over periocular injections as drug molecules are directly inserted into the vitreous chamber. This method involves the injection of a drug solution directly into the vitreous, via the pars plana, using a 30-Ga needle. Unlike other routes, intravitreal injection offers higher drug concentrations in the vitreous and the retina. The advantage of IVT injection is the circumvention of the blood-retinal barrier, which keeps most drugs out of the eye in the case of oral and systemic administrations [43]. Therefore, IVT administration is able to maximize the intraocular level of drugs in the vitreous and the retina while avoiding toxicities associated with systemic treatment [66-69]. The benefits of intravitreal therapies in retinal diseases have been well documented, and the current standard of care is the injection of a bolus of anti-VEGF into the vitreous cavity on a frequent basis. Such a large bolus of a drug has been associated with some side effects [70]. In addition, this administration procedure creates a typical curve with a large peak of drug concentrations and a rapid decay. Literature supports the fact that the half-life of anti-VEGFs, such as ranibizumab, is indeed very short (halflife $=2.6-4.0$ days) [71], making it necessary to use a higher initial dose in order to exceed therapeutic levels so as to allow a longer 28-day treatment interval. Moreover, mathematical modeling demonstrates that the binding activity of $0.5 \mathrm{mg}$ of ranibizumab is fivefold higher if given every 14 days instead of every 28 days [72]. 
Intravitreal injection can avoid ocular barriers for direct drug placement in the vitreous cavity, but drug distribution in the vitreous is not uniform. Small molecules can rapidly be distributed throughout the vitreous, whereas the diffusion of larger molecules is restricted. This distribution also depends on the presence of physiopathological conditions of the vitreous (vitrectomized patients present poor concentrations after 3 days) and the molecular weight of the administered drug [69]. In order to reach and maintain effective therapeutic concentrations, repeated injections are necessary. The frequent administration of drugs via this route can lead to endophthalmitis, damage to the lens, retinal detachment, and hemorrhage. Moreover, high acute intraocular drug concentrations may induce severe local toxicity and increase intraocular pressure. Additionally, it may be a burden for physicians, the health system, and patients with poor compliance in many cases [15]. Furthermore, IVT injections require highly specialized human resources and special infrastructure to apply them, and it is a costly option in developing countries [16].

Due to these limitations, new drug delivery systems are needed in order to find a safer and more effective ocular drug delivery method for the treatment of retinal diseases such as those from intraocular implants. Various drug delivery systems have been proposed using a group of biodegradable and nonbiodegradable platforms to passively deliver anti-angiogenic drugs [73-81]. However, one of the limitations of these drug delivery platforms is that, once implanted, they elute an unchangeable dosage of the drug and can only be stopped by explanting the device. In this context, they also share many of the problems of IVT injections.

\section{Topical nanocarriers for drug delivery into the posterior segment of the eyeball}

To date, different nanocarriers have proven to be capable technologies to circumvent most of the ocular barriers and effectively deliver drugs to the posterior segment of the eye. Their favorable physicochemical properties, such as the small size and adjustable surface, render them advantageous in targeting drugs to the retina. Although different administration routes have been analyzed, we consider that the topical route is the one of greatest interest because of its favorable characteristics such as its ease for the patient, a potentially better compliance, and lower incidence of serious complications than injected methods. The main topically proven nanocarriers are polymeric NPs, lipid nanoparticles, micelles, dendrimers, and liposomes.

\subsection{Polymeric nanoparticles}

Polymeric nanoparticles (PNPs) are colloidal particles $(10-1000 \mathrm{~nm})$ where the drug is uniformly distributed throughout the particle matrix (nanospheres) or encapsulated inside a polymer shell (nanocapsules) [82]. Synthetic and natural biocompatible polymers can be used to develop PNPs. The polymers used to generate PNPs for ocular applications have been made from poly(alkyl cyanoacrylates) $[83,84]$, polycaprolactone, chitosan (CS), hyaluronic acid (HA), Eudragit (RS100 and RL100), Carbopol, gelatin, poly(butyl cyanoacrylate), polylactic acid (PLA), and poly (lactic-co-glycolic acid) (PLGA) [57, 85].

In regard to topical PNPs for posterior segment drug delivery, we would like to highlight the study performed by Tahara $\mathrm{K}$ et al., where submicron-sized poly(D,Llactide-co-glycolide) (PLGA) nanoparticles were used to improve the efficiency of drug delivery to the retina through topically administered drugs. Chitosan and glycol chitosan (GCS), which are mucoadhesive polymers, and polysorbate 80 
(P80) were used as surface modifiers of PGLA nanoparticles since this substance increases the interaction of NPs with cells. Coumarin- 6 was used as a model drug and a fluorescent marker. The in vivo fluorescence image analysis showed detectable fluorescence intensity of coumarin- 6 in the retina of mice after its topical administration [86]. The author proposed that the delivery of coumarin- 6 into the posterior segment was associated with ocular surface modifications induced by CS, GCS, or P80. However, they did not verify this hypothesis.

Another interesting work concerning topically administered PNPs for retinal drug delivery was the study performed by Binstock et al., where the penetration of charged fluorescent nanoparticles into rabbit eyes using hydrogel iontophoresis was evaluated. Polyacrylic hydrogels were loaded with charged nanoparticles. NP suspensions (20-45 nm) were administered using cathodal and anodal iontophoresis and applying a current intensity of $1.5 \mathrm{~mA}$ for $5 \mathrm{~min}$ at the central cornea and the sclera (pars plana). The mean fluorescence intensity in outer ocular tissues (cornea, conjunctiva, and sclera) was 2-3 times higher for positively charged NPs with anodal iontophoresis. In deeper ocular tissues (retina, choroid, ciliary body, and iris), the mean fluorescence intensity was 5-15 times higher for the negatively charged NPs with cathodal iontophoresis. The positively charged particles demonstrated better penetration into inner ocular tissues than negatively charged particles [87]. This work showed that hydrogel iontophoresis combined with charged nanoparticles could be a potential technique for effective retinal drug delivery, but the investigation will have to be broadened and the potential adverse events explored.

\subsection{Lipid nanoparticles}

Three different types of lipid nanoparticles (LNPs) have been used for ophthalmic formulations: solid lipid nanoparticles (SLNPs), nanostructured lipid carriers (NLCs), and hybrid lipid nanoparticles. SLNPs are aqueous colloidal dispersions composed of solid lipids as a matrix material with the capacity to encapsulate hydrophilic and lipophilic drugs. SLNPs are provided with multiple advantages such as physical stability, protection of the encapsulated drug, controlled release, biocompatibility, feasible production, and the possibility of being sterilized by autoclaving [88-91]. On the other hand, SLNs have limited drug loading and expulsion for both hydrophilic and lipophilic drugs. Loaded drugs are usually located between fatty acid chains, in lipid layers, or in crystal imperfections [92]. NLCs consist of a mixture of solid and liquid lipids, and they exhibit a higher drug-loading capacity and longer stability than SLNPs [93]. Finally, hybrid lipid nanoparticles modified by multifunctional polymers [94] combine the merits of polymeric nanoparticles and lipid-based systems, which improve the pharmacokinetics and biodistribution of the loaded drug [95].

Experimentally, LNPs have been used for the delivery of substances to the posterior segment of the eye via corneal and non-corneal pathways. Araujo et al. [96] developed a nanometric ( $200 \mathrm{~nm})$, unimodal, and negatively charged NLC loaded with the fluorescent lipid, Nile red (NR-NLC) marker and a drug, triamcinolone acetonide (TA). The method used for the construction of the NLC was high-pressure homogenization. After NR-NLC eyedrop instillation in mice, lipophilic actives were released into the posterior segment of the eye. Retinal fluorescence of Nile red gradually increased over time, peaking $40 \mathrm{~min}$ after administration and almost disappearing at $160 \mathrm{~min}$. The authors suggested that the fluorescence component of NR-NLC reached the retina through the BRB via non-corneal routes (sclera and conjunctiva).

In the work of Balguri et al., SLNPs and NLCs loaded with indomethacin (IN) were prepared through a hot homogenization method. Additionally, CS was used to modify the surface of SLNPs in order to increase penetration. After the topical application of formulations in rabbits, the concentration of IN was evaluated in 
ocular tissues by using high-performance liquid chromatography (HPLC)-UV method. In vivo bioavailability studies demonstrated that the IN of the formulations (IN-SLNPs, IN-NLC, and IN-CS-NLC) reached the posterior ocular tissues $2 \mathrm{~h}$ posttopical application. Moreover, the IN-NLC showed higher drug-loading capability, higher entrapment efficiency, less drug expulsion, and, especially, the highest distribution concentrations of IN in deeper ocular tissues (vitreous humor, choroidretina, and sclera). In vitro corneal penetration studies showed that CS increased corneal penetration by twofold (IN-CS-NLC compound), possibly thanks to the enhanced interaction with the mucus of the eye, the increased cellular uptake, and even its internalization. On the basis of their results, the authors proposed that the main transport routes of IN-NLC to successfully deliver IN to the posterior segment are the corneal pathway and the conjunctival-scleral pathway [97].

Finally, SLNPs comprising Compritol ${ }^{\circledR} 888$ ATO and a polyethylene glycol (PEG) core, into which KTZ is dissolved, were topically used in rabbits. These SLNPs were generated by using a hot high-pressure homogenization method. Ocular pharmacokinetic studies indicated 2.5-fold and 1.6-fold increases in the aqueous humor and the vitreous humor via the topical administration compared to KTZ suspensions, respectively. Additionally, SLNPs were safe for the ocular surfaces of the study rabbits, and cell viability assays showed nontoxicity [98].

\subsection{Polymeric micelles}

Micelles (10-100 nm) comprise amphiphilic block copolymers above the critical micellar concentration with a hydrophobic core and a hydrophilic shell [99]. The preparation process of colloidal dispersions consists of the exposition of self-assembly oriented amphiphilic molecules in order to form core-shell monomer nanomicelles in a special solvent. These nanodispersions solubilize hydrophobic drugs by entrapping them within a mixed micellar hydrophobic core, resulting in clear aqueous formulations [100]. Micelles prevent or minimize drug degradation, improve drug permeation, increase the solubility and stability of drugs, and improve ocular bioavailability $[101,102]$.

Lately, nanomicelles/mixed nanomicelles loaded with different drugs such as dexamethasone [103,104], voclosporin [105], and rapamycin [106] have been designed to reach the posterior segment of the eye. Liaw et al. [107] also demonstrated that micelles could be effective for intraocular gene delivery. In this work, pDNA with the lacZ gene was coupled to poly(ethylene oxide)-poly(propylene oxide)poly(ethylene oxide) (PEO-PPO-PEO) nonionic copolymeric micelles. Following topical administration, gene expression was detected around the rabbit iris, sclera, and conjunctiva, and it was also seen in the intraocular tissues of nude mice after topical application for $48 \mathrm{~h}$. In another study, Cholkar et al. [108] optimized an aqueous micellar solution of isopropyl ester carrying the resolvin (RX-10045) prodrug. Ocular tissue distribution studies demonstrated significant drug concentrations in anterior ocular tissues. Moreover, RX-10008 (the active metabolite of RX-10045) was detected in the retina/choroid upon topical drop instillation in rabbits. Micelles appeared to follow the conjunctival-scleral pathway to reach the retina, and no evidence of ocular damage after multiple dosing of this micellar solution was observed [108].

\subsection{Dendrimers}

Dendrimers are highly branched treelike structures with a core and many side chain [109] moieties. The size of dendrimer nanoparticles is usually under $100 \mathrm{~nm}$, and peripheral functional groups (neutral, negative, or positive) may suffer secondary surface modifications in order to improve their characteristics for 
ophthalmic use [110]. Due to their enhanced aqueous solubility, a large variety of surface groups, and their nontoxic nature, polyamidoamine (PAMAM) dendrimers have been investigated the most in regard to ocular drug delivery $[111,112]$.

Yavuz et al. [113] generated various anionic dexamethasone (DEX)-PAMAM complexes and evaluated the delivery of DEX to the posterior segment following topical administration in rats. The ocular tissue distribution study in rabbit eyes showed that anionic DEX-PANAM dendrimers reach the highest concentration of DEX in the vitreous body, the retina-choroid, and the sclera compared to topical DEX suspension. In addition, in vitro studies demonstrated that dendrimers with peripheral carboxyl or hydroxyl functional groups rarely showed cytotoxicity. Transport study results showed that dendrimer complexation increases DEX transport across both corneal and scleral tissues. Additionally, dendrimers have proven their efficiency to release genetic material when topically administered [114].

\subsection{Cyclodextrin nanoparticles}

Cyclodextrins (CDs) are a family of natural cyclic oligosaccharides which are usually composed of no more than 20 a-D-glucopyranoside units via the covalent conjugation of $\alpha-1,4$-glycosidic linkages. CDs are cyclic structures whose hydrophilic outer surface is enclosed by the lipophilic internal cavity of low polarity. The features of their special structure predetermined their ease to form inclusion complexes for poorly water-soluble chemicals through non-covalent conjugations (electrostatic interactions, van der Waals contributions, hydrogen bonding, and charge-transfer interaction) [115]. CDs are not toxic for human beings [116], and they can improve the aqueous solubility, stability, activity, and dispersion of ophthalmic drugs [117].

Different types of oligomeric CD molecules are currently being used to produce cyclodextrin-based eye drops. These molecules are the $\beta$-CD [7], the $\gamma$-CD [8], its CD derivatives (hydroxypropyl-beta-cyclodextrin (HP- $\beta-C D)$ ), and the randomly methylated beta-cyclodextrin (RM- $\beta$-CD) [118]. Econazole nitrate (EC) and CD/ HP- $\beta$-CD were complexed to form different EC-CD inclusion complexes through coprecipitation in order to improve ocular bioavailability [119].

In the study of Loftsson et al. [120], inclusion complexes of dexamethasone and $\gamma$-CD were topically administered to rabbits. After $2 \mathrm{~h}$ of application, DEX reached its highest concentration in the vitreous and the retina. The same group performed a clinical trial to prove the efficiency of topical $1.5 \%$ dexamethasone $\gamma$-cyclodextrin nanoparticle eye drops (DexNP) compared to posteriorly the subtenon injection of triamcinolone acetonide in diabetic macular edema (DME) patients. Topical DexNP significantly improved visual acuity and decreased the macular thickness of patients with DME. The effect was similar to that of subtenon triamcinolone. However, a modest increase in intraocular pressure (IOP) was noted with DexNP, but IOP normalized after the discontinuation of the eye drops [21].

\subsection{Liposomes}

Liposomes were described for the first time by Alec Bangham in the 1960s at the Babraham Institute, University of Cambridge, and they consist of single or multiple concentric lipid bilayers encapsulating an aqueous compartment.

Liposomes as carriers for advanced drug delivery have been of much interest lately. They are biocompatible vesicles composed of a phospholipid bilayer with structural resemblance to the cell membrane that form small spheroids that are able to carry both hydrophilic and lipophilic drugs (Figure 2). Phospholipids combined with water immediately form a sphere as one end is water soluble, while the opposite end is water insoluble. Water-soluble medications added to water are 


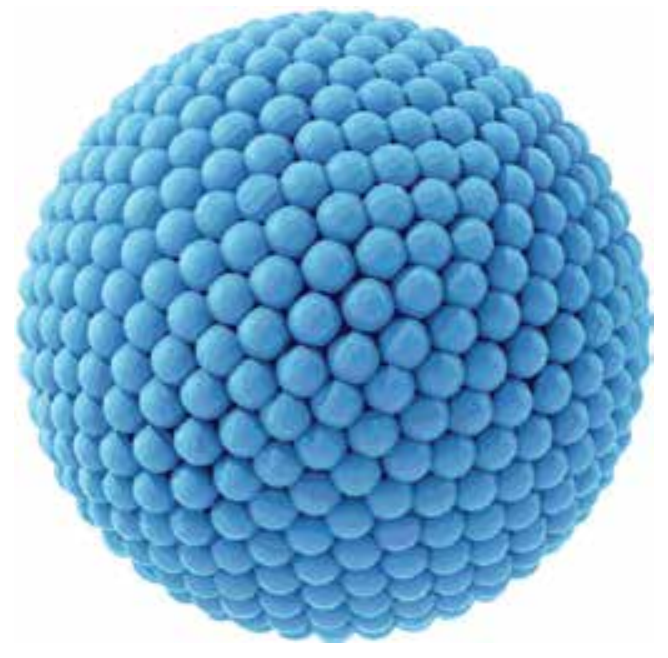

Figure 2.

Spherical formation of liposomes.

trapped inside the aggregation of hydrophobic ends and fat-soluble medications and incorporated into the phospholipid layer. Another significant characteristic of liposomes is their drug release rate, which depends on the liposomal composition, the nature of the drug, and the method of encapsulation.

Liposomes can be composed of naturally derived phospholipids with mixed lipid chains, like that of egg phosphatidylethanolamine, or pure components like dioleoylphosphatidylethanolamine (DOPE). The lipid bilayer can fuse with other bilayers (e.g., the cell membrane), thus delivering the liposomal contents. By including liposomes in solutions with DNA or drugs (which would normally be unable to diffuse through the membrane), they can be delivered past the lipid bilayer. The use of liposomes for the transformation or transfection of DNA into a host cell is known as lipofection. Liposomes can be created by sonicating phospholipids in water. Low shear rates create multilamellar liposomes, which have many layers, like an onion. Continued high-shear sonication tends to form smaller unilamellar liposomes.

\subsubsection{Types of liposomes}

Depending upon their structure, there are two types of liposomes, unilamellar liposomes or unilamellar vesicles, which have a single phospholipid bilayer sphere enclosing an aqueous solution (Figure 3), or multilayer liposomes, which are multilamellar structures (Figure 4). In multilayer liposomes, several unilamellar vesicles will form one inside the other in diminished sizes, creating a multilamellar structure of concentric phospholipid spheres (like a Matryoshka doll) separated by layers of water. The structural components of liposomes could be:

- Phospholipids such as phosphatidylcholine, phosphatidylglycerol, phosphatidylinositol, etc. For stable liposomes, saturated fatty acids are used.

- Sphingolipids like sphingomyelin.

- Sterols, such as cholesterol, with the potential to decrease the fluidity or microviscosity of the bilayer. It can reduce the permeability of the membrane to water-soluble molecules and stabilize the membrane in the presence of biological fluids such as plasma. 


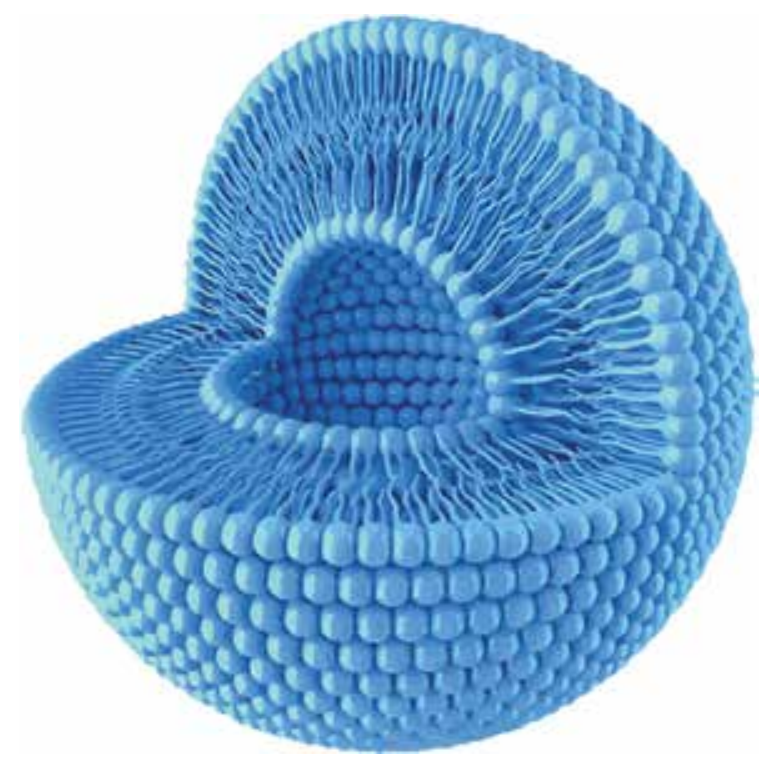

Figure 3.

Single-layer liposome.

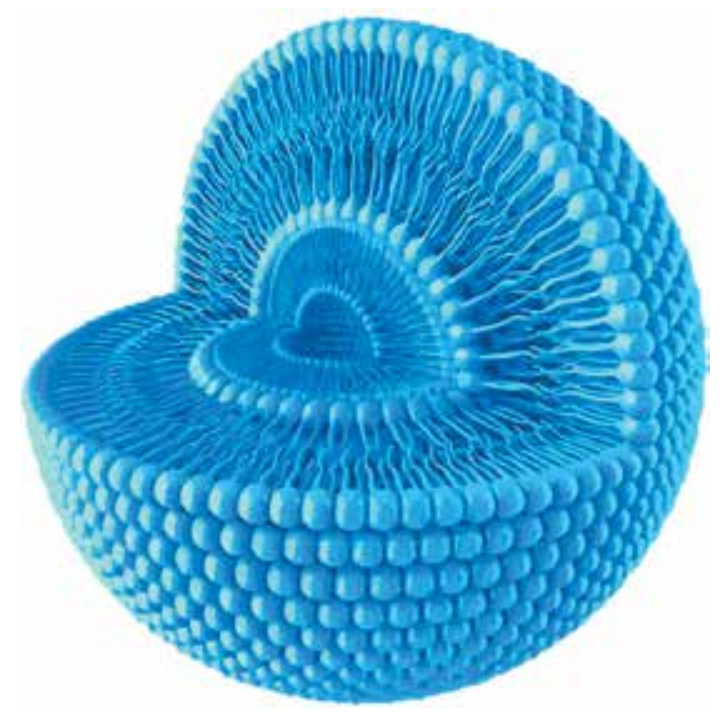

Figure 4.

Multilayer liposome.

- Synthetic phospholipids (saturated and unsaturated).

- Polymeric materials. Polymerized liposomes have significantly higher permeability barriers to entrap aqueous drugs.

- Polymer-bearing lipids. Coating liposome surfaces with charged polymers results in repulsion interactions with macromolecules.

- Cationic lipids such as dioctadecyldimethylammonium bromide or chloride. 
Breaking down the Barrier: Topical Liposomes as Nanocarriers for Drug Delivery...

DOI: http://dx.doi.org/10.5772/intechopen.86601

\subsubsection{Size of liposomes}

Due to differences in preparation methods and lipid compositions, liposomes may be classified, according to their size, in small $(<100 \mathrm{~nm})$, medium (100$250 \mathrm{~nm})$, or large $(>250 \mathrm{~nm})[121,122]$. Some liposomes may be as small as $<0.1 \mu \mathrm{m}$ or as big as $>1 \mathrm{~mm}$ in size.

\subsubsection{Preparation method (incorporating drugs)}

In addition to the ability to entrap drugs with different solubility characteristics, it has been hypothesized that liposomes have different release kinetics. In general, multilamellar liposomes are more easily formed at larger hydrodynamic diameters, and, therefore, they have greater entrapped volumes than unilamellar liposomes. As a result of this, unilamellar liposomes with a hydrodynamic diameter of $130 \mathrm{~nm}$ exhibit a much faster release rate than multilamellar liposomes with two to three lamellar bilayers and a hydrodynamic diameter of $250 \mathrm{~nm}[123,124]$. The difference in the release rate is overall due to the number of phospholipid bilayers that it has to cross before being released $[123,124]$. The ongoing interest of researchers in liposomal characteristics such as their stability, pharmacokinetic properties, and therapeutic efficacy has led to second-generation liposomes through the modulation of their lipid composition, size, and the charge of the vesicle. The addition of cholesterol to the lipid bilayer of liposomes reduces their permeability, increases their in vivo and in vitro stability, and can be used to anchor other molecules such as polyethylene glycol (PEG) or deoxyribonucleic acid (DNA) to the liposomes for their application in biosensing or as "stealth" drug carriers [124]. The use of phosphatidylcholine with saturated fatty acyl chains and materials that stretch transition temperatures beyond $37^{\circ} \mathrm{C}$ offered even greater stabilization [115, 125]. Furthermore, hydrophilic carbohydrates or polymers, such as monosialoganglioside (GM1) and PEG, were included in the liposomal composition. GM1 can lead to the prolongation of the in vivo liposome viability time [126-129].

There are many different methods to prepare liposomes. According to Bozzuto and Molinari [130], the choice of the method depends on factors such as the physicochemical characteristics of liposomes and/or drug components; the toxicity and concentration of the loaded drug; the type of medium in which liposomes are dispersed; the additional process during the application/delivery of liposomes; the desired size for the application; the half-life desired for successful application; costs, reproducibility, and applicability regarding large-scale production for clinical purpose; and good manufacturing practice-relevant issues. In addition, the target organ is a significant issue to be considered when planning the preparation of liposomes.

Every method for preparing liposomes involves four basic stages: drying down lipids from an organic solvent, dispersing the lipid in aqueous media, purifying the resultant liposome, and analyzing the final product. The most common methods for producing liposomes, according to Gabizon et al. [123, 128] and Akbarzadeh et al., [131] are the following.

\section{Mechanical dispersion method}

\subsection{Handshaking and non-handshaking method}

In order to produce liposomes, lipid molecules must be introduced into an aqueous environment. When a dry lipid layer film is hydrated, lamellae swell and grow 
into myelin figures. Mechanical agitation provided by vortexing, shaking, swirling, or pipetting causes myelin figures to break and reseal the exposed hydrophobic edges, resulting in the formation of liposomes, which can be made by using the handshaking method.

\subsection{French pressure cell method}

It involves the extrusion of multilamellar vesicles (MLV) through a small orifice. The method involves the gentle handling of unstable materials. The resulting liposomes are larger than those generated by using the sonicated method. However, high temperatures are difficult to obtain and working volumes are small.

\subsection{Freeze-thawed liposome method}

The creation of unilamellar vesicles is a result of a fusion of UV throughout the processes of rapidly freezing and slowly thawing. The encapsulation rates go from 20 to $30 \%$.

\subsection{The sonication method}

The sonication method is the most used method for the preparation of liposomes (unilamellar). Both techniques are probe sonication, in which the tip sonicator is directly immersed into the liposome. Dispersion is high and there is overheating, so the vessel is submerged in an ice bath. With this technique, up to $5 \%$ of the lipids can be de-esterified after $1 \mathrm{~h}$. The bath sonicator is the second type of sonication method, and the dispersion of liposomes in a tube is placed into a bath sonicator. The regulation of the temperature is easier, and the lipid bilayer of liposomes can fuse with other bilayers, thus delivering the liposomal contents. By making liposomes within drug solutions, they can be delivered past lipid bilayers.

\section{Solvent dispersion method}

\subsection{Ether injection method}

Ether injection method is also known as solvent vaporization. A solution of lipids dissolved in diethyl ether or an ether-methanol mixture is injected into an aqueous solution of the drug in order to be encapsulated at $55-65^{\circ} \mathrm{C}$ or under reduced pressures. The removal of ether under vacuum leads to the formation of liposomes.

\subsection{Ethanol injection method}

A lipid solution of ethanol is rapidly injected to a huge buffer excess. Multilamellar vesicles are at once formed. Unfortunately, the population is not homogeneous with this method.

\subsection{Reverse-phase evaporation method}

Reverse-phase evaporation method is based on the formation of inverted micelles. These inverted micelles are formed upon the sonication of a mixture of a buffered aqueous phase, which contains the water-soluble molecules to be encapsulated into the liposomes, and an organic phase, in which the amphiphilic molecules 
are solubilized. The slow removal of organic solvent leads to the transformation of these inverted micelles into a gel-like, viscous state. After the gel-state collapses, some of the inverted micelles disintegrate, but the excess of phospholipids in the environment contributes to the formation of a complete bilayer around the remaining micelles, which results in the formation of liposomes.

\section{Detergent removal method}

\subsection{Dialysis method}

As the detergent is detached, the micelles increasingly become better-off in phospholipids, and, lastly, they combine to form UVs.

\subsection{Detergent removal of mixed micelle method}

The absorption method is attained by shaking mixed micelle solution with beaded organic polystyrene absorbers.

\subsection{Gel permeation chromatography method}

In this method, the detergent is depleted through size special chromatography.

\subsection{Dilution method}

The spontaneous transition from polydispersed micelles to vesicles occurs due to the dilution of the aqueous micellar solution of detergent and phospholipids with buffer.

\subsection{Stealth liposomes and conventional liposomes}

Liposomes become known by the mononuclear phagocytic system following contact with plasma proteins. This is solved through the use of synthetic phospholipids, particle coated with amphipathic polyethylene glycol, coating liposomes with chitin derivatives, freeze drying, polymerization, and microencapsulation of gangliosides. A stealth liposome is a sphere-shaped vesicle with a membrane that is composed of a phospholipid bilayer used to deliver drugs or genetic material to a cell.

Drug loading can be achieved through passive (if the drug is encapsulated during liposome formation) or active methods (after liposome formation). Freeze-dried (lyophilization) liposomes are formed from preformed liposomes at tremendously low pressures. Very high encapsulation efficiencies, even for macromolecules, can be achieved using this method. During dehydration, the lipid bilayers and the drug to be encapsulated into the liposomes are brought into close contact. Upon reswelling, the chances for the encapsulation of the adhered molecules are much higher. Rehydration is a very important step and it should be very carefully done. The aqueous phase should be added in very small portions with a micropipette to the dried materials. After each addition, the tube should be thoroughly vortexed.

\subsubsection{Transportation mechanisms}

Liposomes can interact with cells through different mechanisms [129]: 
Endocytosis. Carried out by phagocytic cells of the reticuloendothelial system such as macrophages and neutrophils.

Adsorption to the cell surface either through nonspecific weak hydrophobic or electrostatic forces or specific interactions with cell surface components.

Fusion with the plasmatic cell membrane through the insertion of the lipid bilayer of the liposome into the plasma membrane with the simultaneous release of the liposomal content into the cytoplasm.

Transfer of liposomal lipids to cellular or subcellular membranes, or vice versa, without any association of liposomal contents.

\subsubsection{Evaluation}

Liposomal formulation and processing for a specific purpose are characterized for ensuring their predictable in vitro and in vivo performance [132]. Characterization parameters for the purpose of evaluation may be classified into three broad categories that include:

Physical characterization. It evaluates the size, shape, surface features, lamellarity, phase behavior, and drug release profile.

Chemical characterization. It includes studies in order to establish the purity and potency of various lipophilic constituents.

Biological characterization. Biological characterization is helpful in establishing the safety and suitability of the formulation for therapeutic applications.

Some parameters are the vesicle's shape and lamellarity, vesicle size and size distribution, encapsulation efficiency (expressed as percentage (\%)), phase response, and transitional behavior and drug release. Zeta potential (ZP) refers to the potential difference between the electric double layer (EDL) (an adsorbed double layer developed on the surface of dispersed charged particles) of movable particles and the layer of dispersant around them at the slipping plane. A stabilized nanosuspension is a suspension that may be affected by several factors such as $\mathrm{pH}$, ionic strength, and the concentration of particles. The phospholipid composition of liposomes is the main content that influences the overall surface charge of liposomes.

\subsubsection{Applications of liposomes in medicine}

Liposomes are versatile carriers for the delivery of numerous challenging molecules, and they have remarkable advantages compared to other colloidal systems. They have been investigated for a wide range of applications in pharmaceutical technology through topical, transdermal, nasal, and oral routes for efficient and effective drug delivery. Liposome formulations have potential advantages that other drug delivery systems do not have:

i. Suitable for the delivery of hydrophobic, amphipathic, and hydrophilic drugs.

ii. They protect the encapsulated drug from the external environment.

iii. Reduced toxicity and increased stability.

iv. Increased efficacy and therapeutic index of the drug.

v. The sustained-release system of systematically or locally administered liposomes. 
Breaking down the Barrier: Topical Liposomes as Nanocarriers for Drug Delivery...

DOI: http://dx.doi.org/10.5772/intechopen.86601

vi. Improved transfer of hydrophilic, charged molecules.

vii. Improved penetration into the tissues.

viii. Site avoidance mechanism.

ix. They offer site-specific targeting.

x. Nontoxic, flexible, biocompatible, and completely biodegradable.

xi. Size may vary in order to incorporate smaller or larger drug molecules.

xii. They can be administered through various routes.

xiii. The therapeutic activity of chemotherapeutic agents may be improved through liposomal encapsulation. This reduces deleterious effects similar to or lower than those required for maximum therapeutic activity.

xiv. They help reduce exposure of sensitive tissues to toxic drugs.

Some of the disadvantages of liposomes are:

i. Production cost is high.

ii. Leakage and fusion of encapsulated drug molecules with certain preparation methods.

iii. Short half-life.

iv. Less stability.

v. Low solubility.

vi. Phospholipids undergo oxidation and hydrolysis.

vii. Leakage and fusion.

viii. Allergic reactions to liposomal constituents may occur.

Liposomal formulations have several applications in cancer chemotherapy. Due to the nature and behavior of cancer tissues and the large difference between them and regular tissues, cancer tissues are considered an appropriate target for liposome drug delivery systems. For example, the tumor vasculature is characterized by a leaky vasculature and limited lymphatic drainage; consequently, drug molecules can easily be accumulated in intercellular spaces of a large variety of tumors. Numerous different liposome formulations of several anticancer agents were shown to be less toxic than free drugs [131, 133-135]. Examples of these drugs are doxorubicin and daunorubicin citrate for Kaposi sarcoma; doxorubicin for ovarian cancer and solid tumors; nystatin, vinorelbine, cisplatin and its analog docetaxel, tretinoin, siRNA, topotecan, irinotecan, paclitaxel, and camptothecin for solid tumors; cisplatin and its analog for colorectal neoplasms, Grb-2 in leukemia; Bcl-2 for lymphoma; BikDD for pancreatic cancer; and DOTAP (Chol-Fus I) for the 
treatment of lung cancer [130]. Many other drugs are being researched in order to make them affordable for liposomal drug delivery systems.

In dermatology, liposomes have successfully been used in atopic dermatitis (taxifolin glycoside) as antibacterial, antifungal (metronidazole nitrate, amphotericin B), and anti-leishmaniasis treatments (amphotericin B, meglumine antimoniate). Antioxidants such as natural flavonoids (catechin and naringenin) have been used to prevent the oxidation of cutaneous disorders and as photoprotective (quercetin), antipsoriatic, anti-inflammatory agents and anti-acne drugs [136].

In the same context, prostaglandin has been used in peripheral vascular disease, meglumine antimoniate for cutaneous leishmaniasis, fentanyl for pain relief, amikacin in cystic fibrosis, and prilocaine in dental anesthesia, all of them are more examples of liposomal drug products for medical use [136]. Furthermore, antihypertensive drugs have been used for the management of cardiovascular disorders (propanol hydrochloride, valsartan, and nifedipine have been developed in liposomal formulations) [136]. There are publications regarding local anesthetic drugs [137-139], drug delivery to the brain in anti-migraine and anti-Parkinson drugs [140-143], and the nasal delivery of liposomal formulations (salbutamol).

The application of liposomes in vaccine formulations and toxoids is one of the main achievements of modern medicine. Vaccination activates particular parts of the immune system in order for it to express specific immune responses followed by the induction of long-lasting immunological memories to defend against subsequent infectious attacks [144]. Most available immunizations are intramuscularly delivered, which is painful and requires an aseptic technique, as well as skilled and trained personnel for their administration. Thus, biological products (vaccines and toxoids) are suitable as a noninvasive approach compared to conventional methods, and they have numerous obvious advantages such as increased patient compliance, reduced systemic side effects, and constant plasma concentrations. Ding et al. reported on antigens such as vaccines and toxoids. Depending on the type of antigens, the dose to be delivered, immunization schedule, the presence of co-stimulatory factors, and liposomal composition, the immunization of antigens loaded in ELs elicits an effective immune response with serum IgG levels comparable to those obtained after subcutaneous injections [145-148]. Transcutaneous immunization (TCI) is a novel technique, and it requires the simple introduction of antigens into the host tissue through topical application on the skin [148]. This offers ease of administration and the potential to elicit a robust immune response as compared to conventional painful (needle injections) methods prescribed in equivalent doses [145].

Image-guided delivery is another opportunity area for liposomal formulations. Imaging plays an integral part in modern precision and individualized medicine. Wide applications of imaging such as monitoring drug delivery, accurate diagnosis of diseases, determining the response to therapy, and guiding minimally invasive procedures are some of the applications of imaging in the clinic. However, traditional imaging modalities, such as computed tomography (CT), positron-emission tomography (PET), magnetic resonance imaging (MRI), and single-photon emission computed tomography (SPECT), all suffer from target specificity, which limits their clinical utility. Nanoparticles, with their versatility in surface functionalization, provide opportunities to enhance target specificity and label NPs with various isotopes, which enables them to act as contrast agents. Recent developments in multimodality imaging to better diagnose diseases and monitor treatments have embarked on using liposomes as a diagnostic tool. Conjugating liposomes with different labeling probes enable the precise localization of these liposomal formulations by using various modalities such as PET, SPECT, and MRI [149]. 
Glucocorticoids (GC) are one of the most popular and versatile classes of drugs available to treat chronic inflammation and cancer, but side effects and resistance constrain their use. In order to overcome these hurdles, which are often related to the uniform tissue distribution of free GC and their short half-life in biological fluids, new delivery vehicles have been developed, including PEGylated liposomes, polymeric micelles, polymer-drug conjugates, inorganic scaffolds, and hybrid NPs. While each of these nanoformulations has individual drawbacks, they are often superior to free GC in many aspects, including therapeutic efficacy when tested in cell cultures or animal models. The characterization and pharmacokinetics of triamcinolone acetonide-loaded liposomal topical formulations for vitreoretinal drug delivery [150] have been published by Altamirano-Vallejo et al. They showed that a formulation with triamcinolone acetonide-loaded liposomes is feasible and that it has the potential to reach the vitreous cavity with a significant concentration of the drug.

Liposomal vesicles have potential advantages when compared to conventional drug delivery methods. Liposomes in different forms are still one of the most investigated drug delivery carrier systems for the ocular delivery of drugs in both preclinical studies and clinical trials. They seem to be an almost ideal drug-carrier system since their morphology is similar to that of cellular membranes and because of their ability to incorporate various substances. They are valued for their biological and technological advantages as optimal delivery systems for biologically active substances, both in vitro and in vivo, and are considered to be the most successful drug-carrier system known to date [151].

\subsubsection{Liposomes in ophthalmology}

Several liposome formulations have been used in ophthalmology to target the anterior segment of the eye. The use of liposomal formulations to enhance the bioavailability of topical applied acyclovir and ganciclovir has been evaluated with promising results [152]. Antibacterial drugs, such as tetracycline, gentamycin, ciprofloxacin, norfloxacin, and chloramphenicol, have been prepared as liposomal solutions with higher activity compared to that of standard solutions [153]. Antifungal agents such as amphotericin B and fluconazole have been under research [152], and liposomal formulations were highly effective in treating Candida keratitis. Anti-inflammatory drugs and immunomodulatory agents are widely used in the treatment of ocular inflammatory and immunological diseases. In order to enhance ocular bioavailability and reduce the toxic effects following topical or intravitreal administration, liposomal forms of many of these drugs have been evaluated (diclofenac, cyclosporine, tacrolimus) with promising results and better concentrations than standard formulations [152, 153]. Similarly, liposomal antiglaucoma agents' formulations (pilocarpine, latanoprost, acetazolamide) were more effective in reducing intraocular pressure (IOP). In the same context, lubricants and antioxidants have also other potential uses for liposomal formulations.

Liposomal drugs that have transitioned from preclinical research to clinical phase trials include latanoprost-loaded conventional liposomes developed for subconjunctival administration [154]. Phase I and II trials on the safety and efficacy of latanoprost-loaded liposomes in the treatment of ocular hypertension and primary open-angle glaucoma have been completed (ClinicalTrials.gov identifier, NCT0198732357; ClinicalTrials.gov identifier, NCT02466399).

\subsubsection{Topical liposomes for drug delivery into the posterior segment of the eyeball}

Different efforts have been performed to deliver drugs into the posterior segment of the eye through the instillation of loaded liposomes (Table 2) [155-163]. 


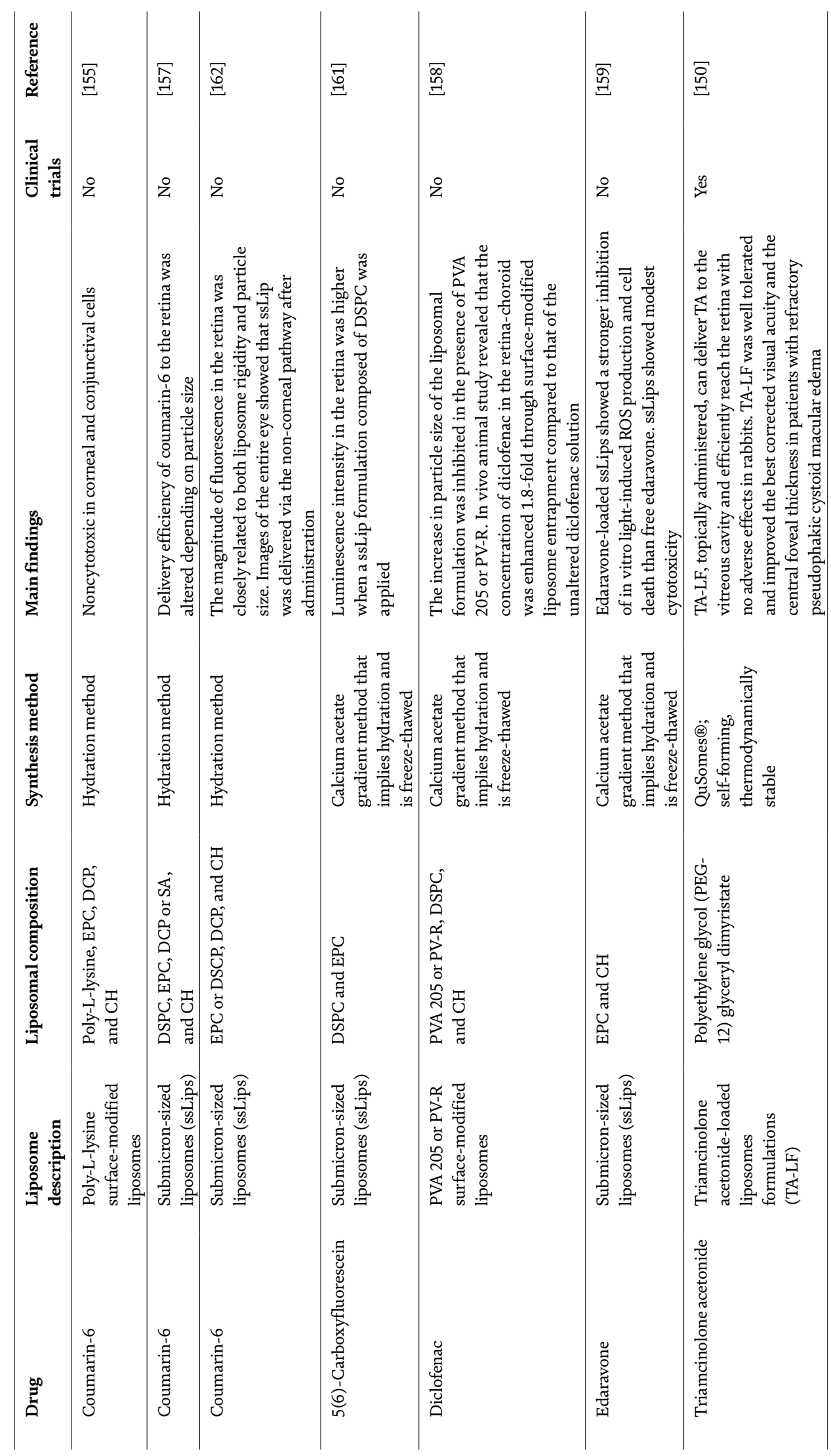


Breaking down the Barrier: Topical Liposomes as Nanocarriers for Drug Delivery... DOI: http://dx.doi.org/10.5772/intechopen.86601

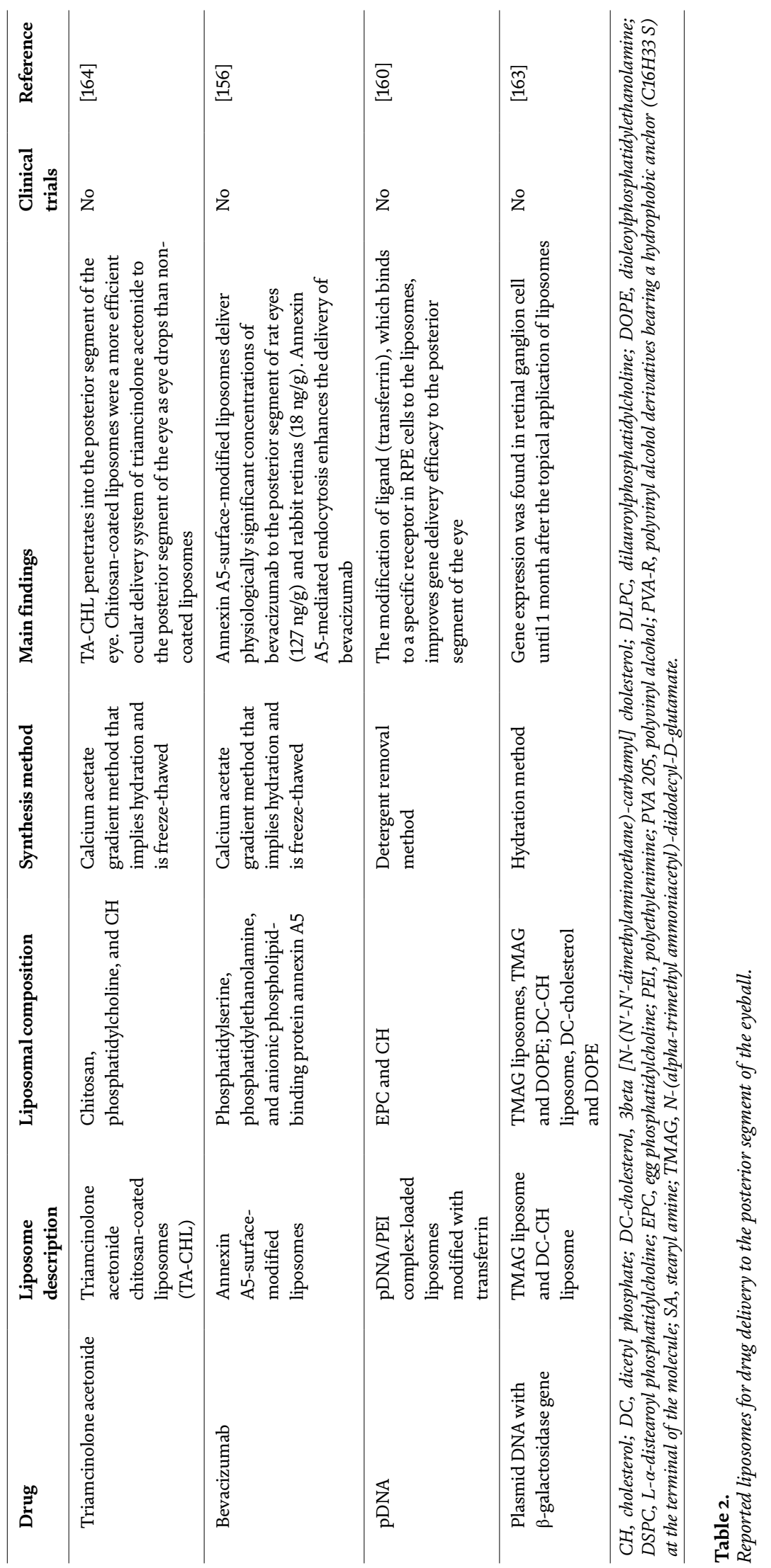


For example, fluorescent probes used as drug models, such as coumarin- 6 and 5(6)-carboxyfluorescein, have efficiently been released into the posterior segment of the eyeball by liposomes $[155,157,161,162]$. On the other hand, drugs like edaravone and diclofenac were successfully released into the vitreous and the retina by liposomes $[158,159]$.

Special mention is reserved for the study performed by Davis BM et al. This group demonstrated that the topical instillation of eye drops containing annexin A5 associated with liposomes loaded with bevacizumab is able to deliver physiologically significant concentrations of this large therapeutic protein (monoclonal antibody against vascular endothelial growth factor A) into the posterior segment of the eye in animal models (rats and rabbits) [156]. Moreover, liposomes can release genetic material into the vitreous and the retina [160,163].

Lastly, a topical triamcinolone acetonide-loaded liposomes formulation (TALF) was used to successfully deliver TA into the vitreous and the retina of rabbits. Besides the authors report that TA-LF was well tolerated by the study animals and that no toxicity was observed in cell culture assays and no adverse events like corneal and conjunctival erosions were observed [150]. Recently, Jin Li et al. [164] validated in animal models (mice) that eye drops containing chitosan-coated liposomes carrying TA are an efficient method to deliver this drug into the posterior segment of rabbit eyeballs, supporting the previous findings published by Altamirano-Vallejo et al. Even though Li et al. reached superior TA entrapment efficiency in their TA-loaded liposomes prepared through the calcium acetate gradient method, it seems that this characteristic does not compromise the therapeutic activity of TA-LF. TA-LF has been tested in clinical assays where its efficiency and safety profile have satisfactorily been demonstrated. In a recent report, TA-LF was efficient in the management of refractory pseudophakic cystoid macular edema [20], where the use of this formulation was associated with the improvement of best corrected visual acuity and central foveal thickness with no reports of adverse events. At this time, phase II trials are underway to demonstrate the efficacy of TA-LF for macular edema.

\section{Conclusion}

Although there has been great interest in the development of new topical ocular delivery systems, the topical administration of drugs is not as effective as intravitreal delivery in treating retinal diseases. During topical delivery, drug molecules must cross several anatomic and physiologic barriers before reaching the posterior segment of the eye. Consequently, very low concentrations, with almost no clinical effect, are usually obtained. However, dramatic changes have been observed in the field of ocular drug delivery over the last decade, and nanomedicine is one of the most promising technologies for efficient drug delivery into intraocular tissues. Nanocarriers have a great potential to solve the challenge of releasing drugs into the posterior segment of the eyeball and reaching deeper intraocular tissues. Different nanocarriers, such as NPs, LPs, micelles, dendrimers, cyclodextrin nanoparticles, and liposomes, have been developed for the safe and effective drug delivery to the vitreous and the retina. Nevertheless, only a few of these particles have clinically been approved (liposomes and CDs among them).

Liposomes have lately been of great interest as carriers for advanced drug delivery in medicine and, especially, in ophthalmology due to their potential to avoid sophisticated ocular barriers when they are topically used. Liposomal drug delivery systems have a bright future in ophthalmology and, particularly, for the retina for several reasons. First, features such as the passive-targeting effect may substantially 
enhance the amount of drug in target tissues. Second, liposomal technology allows the therapeutic index of already established drugs, with well-established therapeutic profiles, to be improved. This eliminates some of the considerable risks associated with the development of new pharmaceutical products. Third, many of the potential new pharmaceutical products arising from advances in biotechnology would come from macromolecules such as proteins, peptides, oligonucleotides, and plasmids, which could easily be carried by liposomes so as to access target tissues and cells. Additionally, liposomes possess features of optimal drug delivery systems to get to the ocular surface and intraocular tissues, for instance, sustained and controlled drug delivery, ease to instill for the patient, good corneal-scleral penetration, and the fact that they reach appropriate concentrations of the drug in the target tissue, avoid side effects produced by conventional systems (like IVT injections), there is better patient compliance, and they are potentially affordable for patients. A proof of the clinical advantages of liposomes is represented by recent trials where TA-loaded liposomes have successfully been used to treat macular edema.

Considering that liposomes collect the main characteristics of an optimal drug delivery system and that these nanocarriers have proven their capacity not only to deliver drugs but also genetic materials to the posterior segment of the eye, we consider that liposomes are promising nanoparticles for the therapy of multiple intraocular illnesses.

\section{Author details}

Arturo Santos ${ }^{1,2 *}$, Juan C. Altamirano-Vallejo ${ }^{1,2}$, José Navarro-Partida ${ }^{1,2}$, Alejandro González-De la Rosa ${ }^{1,2}$ and Jane H. Hsiao ${ }^{3}$

1 Tecnológico de Monterrey, Escuela de Medicina y Ciencias de la Salud, Zapopan, Jalisco, México

2 Centro de Retina Médica y Quirúrgica S.C., Centro Médico Puerta de Hierro, Zapopan, Jalisco, México

3 OPKO Health Inc., Miami, Florida, United States of America

*Address all correspondence to: asantos@e-retina.com

IntechOpen

(C) 2019 The Author(s). Licensee IntechOpen. This chapter is distributed under the terms of the Creative Commons Attribution License (http://creativecommons.org/licenses/ by/3.0), which permits unrestricted use, distribution, and reproduction in any medium, provided the original work is properly cited. (cc) BY 


\section{References}

[1] Jiang S, Franco YL, Zhou Y, Chen J. Nanotechnology in retinal drug delivery. International Journal of Ophthalmology. 2018;11:1038-1044

[2] Kim SH, Lutz RJ, Wang NS, Robinson MR. Transport barriers in transscleral drug delivery for retinal diseases. Ophthalmic Research. 2007;39:244-254

[3] Raghava S, Hammond M, Kompella UB. Periocular routes for retinal drug delivery. Expert Opinion on Drug Delivery. 2004;1:99-114

[4] Ruponen M, Urtti A. Undefined role of mucus as a barrier in ocular drug delivery. European Journal of Pharmaceutics and Biopharmaceutics. 2015;96:442-446

[5] Leong YY, Tong L. Barrier function in the ocular surface: From conventional paradigms to new opportunities. The Ocular Surface. 2015;13:103-109

[6] Grzybowski A, Told R, Sacu S, Bandello F, Moisseiev E, Loewenstein A, et al. 2018 update on intravitreal injections: Euretina expert consensus recommendations. Ophthalmologica. 2018;239:181-193

[7] Young S, Larkin G, Branley M, Lightman S. Safety and efficacy of intravitreal triamcinolone for cystoid macular oedema in uveitis. Clinical \& Experimental Ophthalmology. 2001;29:2-6

[8] Smith JR, Rosenbaum JT, Wilson DJ, Doolittle ND, Siegal T, Neuwelt EA, et al. Role of intravitreal methotrexate in the management of primary central nervous system lymphoma with ocular involvement. Ophthalmology. 2002;109:1709-1716

[9] Davis M. Results of the Endophthalmitis Vitrectomy Study. A randomized trial of immediate vitrectomy and of intravenous antibiotics for the treatment of postoperative bacterial endophthalmitis. Endophthalmitis Vitrectomy Study group. Archives of Ophthalmology. 1995;113:1479-1496

[10] Eyetech Study G. Anti-vascular endothelial growth factor therapy for subfoveal choroidal neovascularization secondary to age-related macular degeneration: Phase II study results. Ophthalmology. 2003;110:979-986

[11] Wu H, Chen TC. The effects of intravitreal ophthalmic medications on intraocular pressure. Seminars in Ophthalmology. 2009;24:100-105

[12] Lyall DA, Tey A, Foot B, Roxburgh ST, Virdi M, Robertson C, et al. Postintravitreal anti-VEGF endophthalmitis in the United Kingdom: Incidence, features, risk factors, and outcomes. Eye. 2012;26:1517-1526

[13] Poku E, Rathbone J, Wong R, Everson-Hock E, Essat M, Pandor A, et al. The safety of intravitreal bevacizumab monotherapy in adult ophthalmic conditions: Systematic review. BMJ Open. 2014;4:e005244

[14] Fung AE, Rosenfeld PJ, Reichel E. The international intravitreal bevacizumab safety survey: Using the internet to assess drug safety worldwide. The British Journal of Ophthalmology. 2006;90:1344-1349

[15] Droege KM, Muether PS, Hermann MM, Caramoy A, Viebahn U, Kirchhof B, et al. Adherence to ranibizumab treatment for neovascular age-related macular degeneration in real life. Graefe's Archive for Clinical and Experimental Ophthalmology. 2013;251:1281-1284

[16] Azad R, Chandra P, Gupta R. The economic implications of the use 
of anti-vascular endothelial growth factor drugs in age-related macular degeneration. Indian Journal of Ophthalmology. 2007;55:441-443

[17] Bisht R, Mandal A, Jaiswal JK, Rupenthal ID. Nanocarrier mediated retinal drug delivery: Overcoming ocular barriers to treat posterior eye diseases. Wiley Interdisciplinary Reviews Nanomedicine and Nanobiotechnology. 2018;10:1-21

[18] Duncan R, Gaspar R. Nanomedicine(s) under the microscope. Molecular Pharmaceutics. 2011;8:2101-2141

[19] Cho K, Wang X, Nie S, Chen ZG, Shin DM. Therapeutic nanoparticles for drug delivery in cancer. Clinical Cancer Research. 2008;14:1310-1316

[20] Gonzalez-De la Rosa A, NavarroPartida J, Altamirano-Vallejo JC, Hernandez-Gamez AG, Garcia-Banuelos JJ, Armendariz-Borunda J, et al. Novel triamcinolone acetonide-loaded liposomes topical formulation for the treatment of cystoid macular edema after cataract surgery: A pilot study. Journal of Ocular Pharmacology and Therapeutics. 2019;35:106-115

[21] Ohira A, Hara K, Johannesson G, Tanito M, Asgrimsdottir GM, Lund SH, et al. Topical dexamethasone gammacyclodextrin nanoparticle eye drops increase visual acuity and decrease macular thickness in diabetic macular oedema. Acta Ophthalmologica. 2015;93:610-615

[22] Gaudana R, Ananthula HK, Parenky A, Mitra AK. Ocular drug delivery. The AAPS Journal. 2010;12:348-360

[23] Barar J, Javadzadeh AR, Omidi Y. Ocular novel drug delivery: Impacts of membranes and barriers. Expert Opinion on Drug Delivery. 2008;5:567-581

[24] Boubriak OA, Urban JP, Akhtar S, Meek KM, Bron AJ. The effect of hydration and matrix composition on solute diffusion in rabbit sclera. Experimental Eye Research. 2000;71:503-514

[25] Hamalainen KM, Kananen K, Auriola S, Kontturi K, Urtti A. Characterization of paracellular and aqueous penetration routes in cornea, conjunctiva, and sclera. Investigative Ophthalmology \& Visual Science. 1997;38:627-634

[26] Kao JC, Geroski DH, Edelhauser HF. Transscleral permeability of fluorescent-labeled antibiotics. Journal of Ocular Pharmacology and Therapeutics. 2005;21:1-10

[27] Shuler RK Jr, Dioguardi PK, Henjy C, Nickerson JM, Cruysberg LP, Edelhauser HF. Scleral permeability of a small, single-stranded oligonucleotide. Journal of Ocular Pharmacology and Therapeutics. 2004;20:159-168

[28] Cruysberg LP, Franklin AJ, Sanders J, Self C, Yuan P, Csaky KG, et al. Effective transscleral delivery of two retinal antiangiogenic molecules: Carboxyamidotriazole (CAI) and 2-methoxyestradiol (2ME2). Retina. 2005;25:1022-1031

[29] Cheruvu NP, Kompella UB. Bovine and porcine transscleral solute transport: Influence of lipophilicity and the choroid-Bruch's layer. Investigative Ophthalmology \& Visual Science. 2006;47:4513-4522

[30] Cruysberg LP, Nuijts RM, Geroski DH, Koole LH, Hendrikse F, Edelhauser HF. In vitro human scleral permeability of fluorescein, dexamethasonefluorescein, methotrexate-fluorescein and rhodamine 6G and the use of a coated coil as a new drug delivery system. Journal of Ocular Pharmacology and Therapeutics. 2002;18:559-569

[31] Prausnitz MR, Noonan JS. Permeability of cornea, sclera, and conjunctiva: A literature analysis 
for drug delivery to the eye. Journal of Pharmaceutical Sciences.

1998;87:1479-1488

[32] Ambati J, Canakis CS, Miller JW, Gragoudas ES, Edwards A, Weissgold DJ, et al. Diffusion of high molecular weight compounds through sclera. Investigative Ophthalmology \& Visual Science. 2000;41:1181-1185

[33] Ahmed I, Gokhale RD, Shah MV, Patton TF. Physicochemical determinants of drug diffusion across the conjunctiva, sclera, and cornea. Journal of Pharmaceutical Sciences. 1987;76:583-586

[34] Olsen TW, Edelhauser HF, Lim JI, Geroski DH. Human scleral permeability. Effects of age, cryotherapy, transscleral diode laser, and surgical thinning. Investigative Ophthalmology \& Visual Science. 1995;36:1893-1903

[35] Larsson BS. Interaction between chemicals and melanin. Pigment Cell Research. 1993;6:127-133

[36] Schoenwald RD, Tandon V, Wurster DE, Barfknecht CF. Significance of melanin binding and metabolism in the activity of 5-acetoxyacetylimino4-methyl-delta2-1,3,4,-thiadiazolin e-2-sulfonamide. European Journal of Pharmaceutics and Biopharmaceutics. 1998;46:39-50

[37] Leblanc B, Jezequel S, Davies T, Hanton G, Taradach C. Binding of drugs to eye melanin is not predictive of ocular toxicity. Regulatory Toxicology and Pharmacology. 1998;28:124-132

[38] Pitkanen L, Ranta VP, Moilanen H, Urtti A. Binding of betaxolol, metoprolol and oligonucleotides to synthetic and bovine ocular melanin, and prediction of drug binding to melanin in human choroid-retinal pigment epithelium. Pharmaceutical Research. 2007;24:2063-2070
[39] Salminen L, Imre G, Huupponen R. The effect of ocular pigmentation on intraocular pressure response to timolol. Acta Ophthalmologica Supplement. 1985;173:15-18

[40] Gipson IK, Argueso P. Role of mucins in the function of the corneal and conjunctival epithelia. International Review of Cytology. 2003;231:1-49

[41] Ahmed I, Patton TF. Importance of the noncorneal absorption route in topical ophthalmic drug delivery. Investigative Ophthalmology \& Visual Science. 1985;26:584-587

[42] Patel A, Cholkar K, Agrahari V, Mitra AK. Ocular drug delivery systems: An overview. World Journal of Pharmacology. 2013;2:47-64

[43] Urtti A. Challenges and obstacles of ocular pharmacokinetics and drug delivery. Advanced Drug Delivery Reviews. 2006;58:1131-1135

[44] Galloway NR. Common eye diseases and their management. American Journal of Ophthalmology. 1985;100:360

[45] Ng J. Ocular Anatomy and Physiology; Thorofare, NJ: SLACK, Incorporated; 2009

[46] Djebli N, Khier S, Griguer F, Coutant AL, Tavernier A, Fabre G, et al. Ocular drug distribution after topical administration: Population pharmacokinetic model in rabbits. European Journal of Drug Metabolism and Pharmacokinetics. 2017;42:59-68

[47] Agrahari V, Mandal A, Agrahari V, Trinh HM, Joseph M, Ray A, et al. A comprehensive insight on ocular pharmacokinetics. Drug Delivery and Translational Research. 2016;6:735-754

[48] Gruntzig J, Schicha H, Kiem J, Becker V, Feinendegen LE. Studies on the lymph drainage of the eye 5 . 
Quantitative registration of the lymph drainage from the subconjunctival space with a radioactive tracer (author's transl). Klinische Monatsblätter für Augenheilkunde. 1978;172:872-876

[49] Collin HB. Lymphatic drainage of 131-I-albumin from the vascularized cornea. Investigative Ophthalmology. 1970;9:146-155

[50] Amrite AC, Kompella UB. Sizedependent disposition of nanoparticles and microparticles following subconjunctival administration. The Journal of Pharmacy and Pharmacology. 2005;57:1555-1563

[51] Toris CB, Yablonski ME, Wang YL, Camras CB. Aqueous humor dynamics in the aging human eye. American Journal of Ophthalmology. 1999;127:407-412

[52] Bill A. A method to determine osmotically effective albumin and gammaglobulin concentrations in tissue fluids, its application to the uvea and a note on the effects of capillary "leaks" on tissue fluid dynamics. Acta Physiologica Scandinavica. 1968;73:511-522

[53] Bill A. Blood circulation and fluid dynamics in the eye. Physiological Reviews. 1975;55:383-417

[54] Kennedy BG, Mangini NJ.

P-glycoprotein expression in human retinal pigment epithelium. Molecular Vision. 2002;8:422-430

[55] Steuer H, Jaworski A, Elger B, Kaussmann M, Keldenich J, Schneider $\mathrm{H}$, et al. Functional characterization and comparison of the outer blood-retina barrier and the blood-brain barrier. Investigative Ophthalmology \& Visual Science. 2005;46:1047-1053

[56] Zhang N, Kannan R, Okamoto CT, Ryan SJ, Lee VH, Hinton DR. Characterization of brimonidine transport in retinal pigment epithelium. Investigative Ophthalmology \& Visual Science. 2006; 47:287-294
[57] Kumari A, Yadav SK, Yadav SC. Biodegradable polymeric nanoparticles based drug delivery systems. Colloids and Surfaces. B, Biointerfaces. 2010;75:1-18

[58] Dhananjay P. Biology of Ocular Transporters: Efflux and Influx Transporters in the Eye. Sawstone, Cambridge: Woodhead Publishing; 2013

[59] Wrighton SA, Thummel KE.

CYP3A. In: Metabolic Drug Interactions. Philadelphia: Lippincott Williams and Wilkins; 2000

[60] Lee VH, Robinson JR. Topical ocular drug delivery: Recent developments and future challenges. Journal of Ocular Pharmacology. 1986;2:67-108

[61] Hughes PM, Olejnik O, Chang-Lin JE, Wilson CG. Topical and systemic drug delivery to the posterior segments. Advanced Drug Delivery Reviews. 2005;57:2010-2032

[62] Duvvuri S, Majumdar S, Mitra AK. Drug delivery to the retina: Challenges and opportunities. Expert Opinion on Biological Therapy. 2003;3:45-56

[63] Ghate D, Edelhauser HF. Ocular drug delivery. Expert Opinion on Drug Delivery. 2006;3:275-287

[64] Ghate D, Brooks W, McCarey BE, Edelhauser HF. Pharmacokinetics of intraocular drug delivery by periocular injections using ocular fluorophotometry. Investigative Ophthalmology \& Visual Science. 2007;48:2230-2237

[65] Castellarinand A, Pieramici DJ. Anterior segment complications following periocular and intraocular injections. Ophthalmology Clinics of North America. 2004;17:583-590

[66] Heinemann MH. Long-term intravitreal ganciclovir therapy for cytomegalovirus retinopathy. Archives of Ophthalmology. 1989;107:1767-1772 
[67] Jacobson MA, O’Donnell JJ, Mills J. Foscarnet treatment of cytomegalovirus retinitis in patients with the acquired immunodeficiency syndrome. Antimicrobial Agents and Chemotherapy. 1989;33:736-741

[68] Thrimawithana TR, Young S, Bunt CR, Green C, Alany RG. Drug delivery to the posterior segment of the eye. Drug Discovery Today. 2011;16:270-277

[69] Mitra AK, Anand BS, Duvvuri S. Drug delivery to the eye. In: The Biology of Eye. New York: Academic Press; 2006

[70] Meyer CH, Michels S, Rodrigues EB, Hager A, Mennel S, Schmidt JC, et al. Incidence of rhegmatogenous retinal detachments after intravitreal antivascular endothelial factor injections. Acta Ophthalmologica. 2011;89:70-75

[71] Gaudreault J, Fei D, Rusit J, Suboc P, Shiu V. Preclinical pharmacokinetics of Ranibizumab (rhuFabV2) after a single intravitreal administration. Investigative Ophthalmology \& Visual Science. 2005;46:726-733

[72] Stewart MW, Rosenfeld PJ, Penha FM, Wang F, Yehoshua Z, Bueno-Lopez E, et al. Pharmacokinetic rationale for dosing every 2 weeks versus 4 weeks with intravitreal ranibizumab, bevacizumab, and aflibercept (vascular endothelial growth factor trap-eye). Retina. 2012;32:434-457

[73] Zhang K, Zhang L, Weinreb RN. Ophthalmic drug discovery: Novel targets and mechanisms for retinal diseases and glaucoma. Nature Reviews Drug Discovery. 2012;11:541-559

[74] Okabe K, Kimura H, Okabe J, Kato A, Kunou N, Ogura Y. Intraocular tissue distribution of betamethasone after intrascleral administration using a nonbiodegradable sustained drug delivery device. Investigative Ophthalmology \& Visual Science. 2003;44:2702-2707
[75] Yasukawa T, Ogura Y, Sakurai E, Tabata Y, Kimura H. Intraocular sustained drug delivery using implantable polymeric devices. Advanced Drug Delivery Reviews. 2005;57:2033-2046

[76] Daull P, Paterson CA, Kuppermann $\mathrm{BD}$, Garrigue JS. A preliminary evaluation of dexamethasone palmitate emulsion: A novel intravitreal sustained delivery of corticosteroid for treatment of macular edema. Journal of Ocular Pharmacology and Therapeutics. 2013;29:258-269

[77] Haghjou N, Soheilian M, Abdekhodaie MJ. Sustained release intraocular drug delivery devices for treatment of uveitis. Journal of Ophthalmic Vision Research. 2011;6:317-329

[78] Hunter RS, Lobo AM.

Dexamethasone intravitreal implant for the treatment of noninfectious uveitis. Clinical Ophthalmology. 2011;5:1613-1621

[79] London NJ, Chiang A, Haller JA. The dexamethasone drug delivery system: Indications and evidence. Advances in Therapy. 2011;28:351-366

[80] Schwartz SG, Flynn HW Jr. Fluocinolone acetonide implantable device for diabetic retinopathy. Current Pharmaceutical Biotechnology. 2011;12:347-351

[81] Campochiaro PA, Hafiz G, Shah SM, Bloom S, Brown DM, Busquets M, et al. Sustained ocular delivery of fluocinolone acetonide by an intravitreal insert. Ophthalmology. 2010;117:1393-1399

[82] Birch DG, Liang FQ. Age-related macular degeneration: A target for nanotechnology derived medicines. International Journal of Nanomedicine. 2007;2:65-77

[83] Harmia T, Speiser P, Kreuter J. A solid colloidal drug delivery 
system for the eye: Encapsulation of pilocarpine in nanoparticles. Journal of Microencapsulation. 1986;3:3-12

[84] Harmia TKJ, Speiser P, Boye T, Gurny R, Kubis A. Enhancement of the myotic response of rabbits with pilocarpine-loaded polybutylcyanoacrylate nanoparticles. International Journal of Pharmaceutics. 1986;33:87-193

[85] Kimura H, Ogura Y. Biodegradable polymers for ocular drug delivery. Ophthalmologica. 2001;215:143-155

[86] Kohei Tahara KK, Onodera R, Takeuchi H. Feasibility of drug delivery to the eye's posterior segment by topical instillation of PLGA nanoparticles. Asian Journal of Pharmaceutical Sciences. 2017;12:394-399

[87] Eljarrat-Binstock E, Orucov F, Aldouby Y, Frucht-Pery J, Domb AJ. Charged nanoparticles delivery to the eye using hydrogel iontophoresis. Journal of Controlled Release. 2008;126:156-161

[88] Almeida AJ, Souto E. Solid lipid nanoparticles as a drug delivery system for peptides and proteins. Advanced Drug Delivery Reviews. 2007;59:478-490

[89] Joshi MD, Muller RH. Lipid nanoparticles for parenteral delivery of actives. European Journal of Pharmaceutics and Biopharmaceutics. 2009;71:161-172

[90] Beloqui A, Solinis MA, Rodriguez-Gascon A, Almeida AJ, Preat V. Nanostructured lipid carriers: Promising drug delivery systems for future clinics. Nanomedicine. 2016;12:143-161

[91] Wissing SA, Kayser O, Muller RH. Solid lipid nanoparticles for parenteral drug delivery. Advanced Drug Delivery Reviews. 2004;56:1257-1272
[92] Mukherjee S, Ray S, Thakur RS. Solid lipid nanoparticles: A modern formulation approach in drug delivery system. Indian Journal of Pharmaceutical Sciences. 2009;71:349-358

[93] Tian B, Luo Q, Song S, Liu D, Pan H, Zhang W, et al. Novel surfacemodified nanostructured lipid carriers with partially deacetylated watersoluble chitosan for efficient ocular delivery. Journal of Pharmaceutical Sciences. 2012;101:1040-1049

[94] Teixeira MC, Carbone C, Souto EB. Beyond liposomes: Recent advances on lipid based nanostructures for poorly soluble/poorly permeable drug delivery. Progress in Lipid Research. 2017;68:1-11

[95] Souto EB, Doktorovova S, Gonzalez-Mira E, Egea MA, Garcia ML. Feasibility of lipid nanoparticles for ocular delivery of anti-inflammatory drugs. Current Eye Research. 2010;35:537-552

[96] Araujo J, Nikolic S, Egea MA, Souto EB, Garcia ML. Nanostructured lipid carriers for triamcinolone acetonide delivery to the posterior segment of the eye. Colloids and Surfaces. B, Biointerfaces. 2011;88:150-157

[97] Balguri SP, Adelli GR, Majumdar S. Topical ophthalmic lipid nanoparticle formulations (SLN, NLC) of indomethacin for delivery to the posterior segment ocular tissues. European Journal of Pharmaceutics and Biopharmaceutics. 2016;109:224-235

[98] Kakkar S, Karuppayil SM, Raut JS, Giansanti F, Papucci L, Schiavone N, et al. Lipid-polyethylene glycol based nano-ocular formulation of ketoconazole. International Journal of Pharmaceutics. 2015;495:276-289

[99] Nishiyama N, Kataoka K. Current state, achievements, and future prospects of polymeric micelles as 
nanocarriers for drug and gene delivery. Pharmacology \& Therapeutics.

2006;112:630-648

[100] Vadlapudi AD, Mitra AK.

Nanomicelles: An emerging platform for drug delivery to the eye. Therapeutic Delivery. 2013;4:1-3

[101] Cholkar K, Patel A, Vadlapudi AD, Mitra AK. Novel nanomicellar formulation approaches for anterior and posterior segment ocular drug delivery. Recent Patents on Nanomedicine. 2012;2:82-95

[102] Vaishya RD, Khurana V, Patel S, Mitra AK. Controlled ocular drug delivery with nanomicelles. Wiley Interdisciplinary Reviews. Nanomedicine and Nanobiotechnology. 2014;6:422-437

[103] Earla R, Boddu SH, Cholkar K, Hariharan S, Jwala J, Mitra AK. Development and validation of a fast and sensitive bioanalytical method for the quantitative determination of glucocorticoids-quantitative measurement of dexamethasone in rabbit ocular matrices by liquid chromatography tandem mass spectrometry. Journal of Pharmaceutical and Biomedical Analysis. 2010;52:525-533

[104] Chopra P, Hao J, Li SK. Sustained release micellar carrier systems for iontophoretic transport of dexamethasone across human sclera. Journal of Controlled Release. 2012;160:96-104

[105] Velagaleti PR, Anglade E, Khan IJ, et al. Topical delivery of hydrophobic drugs using a novel mixed nanomicellar technology to treat diseases of the anterior \& posterior segments of the eye. Journal of Drug Delivery Science and Technology. 2010;10:42-47

[106] Cholkar K, Gunda S, Earla R, Pal D, Mitra AK. Nanomicellar topical aqueous drop formulation of Rapamycin for Back-of-the-eye delivery. AAPS PharmSciTech. 2015;16:610-622

[107] Liaw J, Chang SF, Hsiao FC. In vivo gene delivery into ocular tissues by eye drops of poly(ethylene oxide)-poly(propylene oxide)poly (ethylene oxide) (PEO-PPO-PEO) polymeric micelles. Gene Therapy. 2001;8:999-1004

[108] Cholkar K, Gilger BC, Mitra AK. Topical delivery of aqueous micellar resolvin E1 analog (RX-10045). International Journal of Pharmaceutics. 2016;498:326-334

[109] Rodriguez Villanueva J, Navarro MG, Rodriguez Villanueva L. Dendrimers as a promising tool in ocular therapeutics: Latest advances and perspectives. International Journal of Pharmaceutics. 2016;511:359-366

[110] Alhalafi AM. Applications of polymers in intraocular drug delivery systems. Oman Journal of Ophthalmology. 2017;10:3-8

[111] Kompella UB, Amrite AC, Pacha Ravi R, Durazo SA. Nanomedicines for back of the eye drug delivery, gene delivery, and imaging. Progress in Retinal and Eye Research. 2013;36:172-198

[112] Chaplot SP, Rupenthal ID. Dendrimers for gene delivery-A potential approach for ocular therapy? The Journal of Pharmacy and Pharmacology. 2014;66:542-556

[113] Yavuz B, Pehlivan SB, Vural I, Unlu N. In vitro/In vivo evaluation of dexamethasone-PAMAM dendrimer complexes for retinal drug delivery. Journal of Pharmaceutical Sciences. 2015;104:3814-3823

[114] Liu C, Jiang K, Tai L, Liu Y, Wei G, $\mathrm{Lu} W$, et al. Facile noninvasive retinal gene delivery enabled by penetratin. 
ACS Applied Materials \& Interfaces. 2016;8:19256-19267

[115] Hosta-Rigau L, Zhang Y, Teo BM, Postma A, Stadler B. Cholesterol-A biological compound as a building block in bionanotechnology. Nanoscale. 2013;5:89-109

[116] Kurkov SV, Loftsson T. Cyclodextrins. International Journal of Pharmaceutics. 2013;453:167-180

[117] Rahim MMF, Nouar L, et al. Driving forces and electronic structure in $\beta$-cyclodextrin $/ 3,3^{\prime}$ diaminodiphenylsulphone complex. Journal of Molecular Liquids. 2014;199:501-510

[118] Loftsson T, Stefansson E. Cyclodextrins and topical drug delivery to the anterior and posterior segments of the eye. International Journal of Pharmaceutics. 2017;531:413-423

[119] Abd El-Gawad AEH, Soliman OA, El-Dahan MS, Al-Zuhairy SAS. Improvement of the ocular bioavailability of econazole nitrate upon complexation with cyclodextrins. AAPS PharmSciTech. 2017;18:1795-1809

[120] Loftsson T, Hreinsdottir D, Stefansson E. Cyclodextrin microparticles for drug delivery to the posterior segment of the eye: Aqueous dexamethasone eye drops. The Journal of Pharmacy and Pharmacology. 2007;59:629-635

[121] Jesorka A, Orwar O. Liposomes: Technologies and analytical applications. Annual Review of Analytical Chemistry. 2008;1:801-832

[122] Makino KS. Chapter 2: Surface Properties of Liposomes Depending on their Composition. Netherland: Elsevier Science; 2006

[123] Betageri GV, Parsons DL. Drug encapsulation and release from multilamellar and unilamellar liposomes. International Journal of Pharmaceutics. 1192;81:235-241

[124] Niven RW, Speer M, Schreier H. Nebulization of liposomes. II. The effects of size and modeling of solute release profiles. Pharmaceutical Research. 1991;8:217-221

[125] Schechter E. Aspects structuraux et fonctionnels. Paris: Dunod; 2002

[126] Bitounis D, Fanciullino R, Iliadis A, Ciccolini J. Optimizing druggability through liposomal formulations: New approaches to an old concept. ISRN Pharmaceutics. 2012;2012:738432

[127] Milla P, Dosio F, Cattel L. PEGylation of proteins and liposomes: A powerful and flexible strategy to improve the drug delivery. Current Drug Metabolism. 2012;13:105-119

[128] Gabizon A, Papahadjopoulos D. Liposome formulations with prolonged circulation time in blood and enhanced uptake by tumors. Proceedings of the National Academy of Sciences of the United States of America. 1988;85:6949-6953

[129] Sharma S, Sharma N, Sandeep K, et al. Liposomes: A review. Journal of Pharmacy Research. 2009;2:1163-1167

[130] Bozzuto G, Molinari A. Liposomes as nanomedical devices. International Journal of Nanomedicine. 2015;10:975-999

[131] Akbarzadeh A, Rezaei-Sadabady R, Davaran S, Joo SW, Zarghami N, Hanifehpour Y, et al. Liposome: Classification, preparation, and applications. Nanoscale Research Letters. 2013;8:102

[132] Kant Shashi KS, Bharat P. A complete review on: Liposomes. International Research Journal of Pharmacy. 2012;3:10-16 
[133] Gabizon A. Liposomes as a drug delivery system in cancer chemotherapy. Horizons in Biochemistry and Biophysics. 1989;9:185-211

[134] Storm G, Roerdink FH, Steerenberg PA, de Jong WH, Crommelin DJ. Influence of lipid composition on the antitumor activity exerted by doxorubicincontaining liposomes in a rat solid tumor model. Cancer Research. 1987;47:3366-3372

[135] Akbarzadeh A, Mikaeili H, Zarghami N, Mohammad R, Barkhordari A, Davaran S. Preparation and in vitro evaluation of doxorubicin-loaded $\mathrm{Fe}(3) \mathrm{O}$ (4) magnetic nanoparticles modified with biocompatible copolymers. International Journal of Nanomedicine. 2012;7:511-526

[136] Hussain A, Singh S, Sharma D, Webster TJ, Shafaat K, Faruk A. Elastic liposomes as novel carriers: Recent advances in drug delivery. International Journal of Nanomedicine. 2017;12:5087-5108

[137] Palac Z, Hurler J, Skalko-Basnet N, Filipovic-Grcic J, Vanic Z. Elastic liposomes-in-vehicle formulations destined for skin therapy: The synergy between type of liposomes and vehicle. Drug Development and Industrial Pharmacy. 2015;41:1247-1253

[138] Cereda CM, Franz-Montan M, da Silva CM, Casadei BR, Domingues CC, Tofoli GR, et al. Transdermal delivery of butamben using elastic and conventional liposomes. Journal of Liposome Research. 2013;23:228-234

[139] Li L, Zhang Y, Han S, Qu Z, Zhao J, Chen $Y$, et al. Penetration enhancement of lidocaine hydrochloride by a novel chitosan coated elastic liposome for transdermal drug delivery. Journal of Biomedical Nanotechnology. 2011;7:704-713
[140] Illum L. Transport of drugs

from the nasal cavity to the central nervous system. European Journal of Pharmaceutical Sciences. 2000;11:1-18

[141] Thorne RG, Pronk GJ, Padmanabhan V, Frey WH 2nd. Delivery of insulin-like growth factor-I to the rat brain and spinal cord along olfactory and trigeminal pathways following intranasal administration. Neuroscience. 2004;127:481-496

[142] Garg T, Jain S, Singh HP, Sharma A, Tiwary AK. Elastic liposomal formulation for sustained delivery of antimigraine drug: In vitro characterization and biological evaluation. Drug Development and Industrial Pharmacy. 2008;34:1100-1110

[143] Villalon CM, Centurion D, Valdivia LF, de Vries P, Saxena PR. Migraine: Pathophysiology, pharmacology, treatment and future trends. Current Vascular Pharmacology. 2003;1:71-84

[144] Foged C, Sundblad A, Hovgaard L. Targeting vaccines to dendritic cells. Pharmaceutical Research. 2002;19:229-238

[145] Ding Z, Bivas-Benita M, Hirschberg H, Kersten GF, Jiskoot W, Bouwstra JA. Preparation and characterization of diphtheria toxoid-loaded elastic vesicles for transcutaneous immunization. Journal of Drug Targeting. 2008;16:555-563

[146] Shi Z, Curiel DT, Tang DC. DNAbased non-invasive vaccination onto the skin. Vaccine. 1999;17:2136-2141

[147] Singh RPSP, Mishra V, et al. Vesicular systems for non-invasive topical immunization: Rationale and prospects. Indian Journal of Biotechnology. 2002;3:9-21

[148] Gupta PN, Singh P, Mishra V, et al. Topical immunization: Mechanistic insight and novel delivery systems. 
Indian Journal of Biotechnology. 2004;3:9-21

[149] Lamichhane N, Udayakumar TS, D'Souza WD, Simone CB 2nd, Raghavan SR, Polf J, et al. Liposomes: Clinical applications and potential for image-guided drug delivery. Molecules. 2018;23:1-17

[150] Altamirano-Vallejo JC, NavarroPartida J, Gonzalez-De la Rosa A, Hsiao $\mathrm{JH}$, Olguin-Gutierrez JS, GonzalezVillegas AC, et al. Characterization and pharmacokinetics of triamcinolone acetonide-loaded liposomes topical formulations for vitreoretinal drug delivery. Journal of Ocular Pharmacology and Therapeutics. 2018;34:416-425

[151] Felice B, Prabhakaran MP, Rodriguez AP, Ramakrishna S. Drug delivery vehicles on a nanoengineering perspective. Materials Science \& Engineering. C, Materials for Biological Applications. 2014;41:178-195

[152] Agarwal R, Iezhitsa I, Agarwal P, Abdul Nasir NA, Razali N, Alyautdin R, et al. Liposomes in topical ophthalmic drug delivery: An update. Drug Delivery. 2016;23:1075-1091

[153] Ahmed KS, Hussein SA, Ali AH, Korma SA, Lipeng Q, Jinghua C. Liposome: Composition, characterisation, preparation, and recent innovation in clinical applications. Journal of Drug Targeting. 2018;15:1-20

[154] Natarajan JV, Ang M, Darwitan A, Chattopadhyay S, Wong TT, Venkatraman SS. Nanomedicine for glaucoma: Liposomes provide sustained release of latanoprost in the eye. International Journal of Nanomedicine. 2012;7:123-131

[155] Sasaki H, Karasawa K, Hironaka K, Tahara K, Tozuka Y, Takeuchi H.
Retinal drug delivery using eye drop preparations of poly-L-lysine-modified liposomes. European Journal of Pharmaceutics and Biopharmaceutics. 2013;83:364-369

[156] Davis BM, Normando EM, Guo L, Turner LA, Nizari S, O’Shea P, et al. Topical delivery of Avastin to the posterior segment of the eye in vivo using annexin A5-associated liposomes. Small. 2014;10:1575-1584

[157] Inokuchi Y, Hironaka K, Fujisawa T, Tozuka Y, Tsuruma K, Shimazawa $\mathrm{M}$, et al. Physicochemical properties affecting retinal drug/coumarin- 6 delivery from nanocarrier systems via eye drop administration. Investigative Ophthalmology \& Visual Science. 2010;51:3162-3170

[158] Fujisawa T, Miyai H, Hironaka K, Tsukamoto T, Tahara K, Tozuka Y, et al. Liposomal diclofenac eye drop formulations targeting the retina: Formulation stability improvement using surface modification of liposomes. International Journal of Pharmaceutics. 2012;436:564-567

[159] Shimazaki H, Hironaka K, Fujisawa T, Tsuruma K, Tozuka Y, Shimazawa $\mathrm{M}$, et al. Edaravone-loaded liposome eye drops protect against light-induced retinal damage in mice. Investigative Ophthalmology \& Visual Science. 2011;52:7289-7297

[160] Takashima Y, Tsuchiya T, Igarashi Y, Kanazawa T, Okada H, Urtti A. Non-invasive ophthalmic liposomes for nucleic acid delivery to posterior segment of eye. Yakugaku Zasshi. 2012;132:1365-1370

[161] Hironaka K, Fujisawa T, Sasaki H, Tozuka Y, Tsuruma K, Shimazawa $\mathrm{M}$, et al. Fluorescence investigation of the retinal delivery of hydrophilic compounds via liposomal eye drops. Biological \& Pharmaceutical Bulletin. 2011;34:894-897 
[162] Hironaka K, Inokuchi Y, Tozuka

Y, Shimazawa M, Hara H, Takeuchi

$\mathrm{H}$. Design and evaluation of a liposomal delivery system targeting the posterior segment of the eye. Journal of Controlled Release. 2009;136:247-253

[163] Masuda I, Matsuo T, Yasuda T, Matsuo N. Gene transfer with liposomes to the intraocular tissues by different routes of administration. Investigative Ophthalmology \& Visual Science. 1996;37:1914-1920

[164] Li J, Cheng T, Tian Q, Cheng Y, Zhao L, Zhang X, et al. A more efficient ocular delivery system of triamcinolone acetonide as eye drop to the posterior segment of the eye. Drug Delivery. 2019;26:188-198 


\title{
Lipid Polymer Hybrid Nanoparticles: A Novel Approach for Drug Delivery
}

\author{
Nayab Tahir, Muhammad Tahir Haseeb, Asadullah Madni, \\ Farzana Parveen, Muhammad Muzamil Khan, \\ Safiullah Khan, Nasrullah Jan and Arshad Khan
}

\begin{abstract}
Applications of nanotechnology and material sciences emerge in the development of various novel drug delivery systems that have been proven as promising clinically. Among these, liposomes, noisome, polymeric carriers and lipid-based delivery system were extensively explored and enter into clinical trials and clinical applications. However, each system has its own pros and cons in term of different physicochemical, pharmacokinetics and therapeutics aspects. Lipid-polymer hybrid carriers merge the potential benefit of these structural components and can be prepared by different approaches to improve the therapeutic outcomes. In this chapter, we provide the useful insight about the lipid-polymer hybrid nanoparticles (LPHNPs) that can be prepared by using the different structural components including the synthetic and natural polymers and lipids. Among these, we also explain the various methods to prepare the LPHNPs with various desired characteristics. Finally, the various therapeutic and clinical applications have been presented briefly.
\end{abstract}

Keywords: lipid-polymer hybrid, nanoparticles, drug delivery, targeted release

\section{Introduction}

The advances in the field of nanotechnology and the material sciences have been open a new horizon for the development of various drug delivery systems (DDS) for the effective and efficient delivery of therapeutic and diagnostic agents [1]. During last few decades, many new DDS have been explored in term of their structural components and medical applications. These systems enhance the application of novel approaches toward the translational medicines by improving the preparation techniques and combing the natural and synthetic polymers and materials [2]. Formulation of these DDS helps to encapsulate a variety of chemotherapeutic agents, vaccines, proteins, antibodies, nucleic acids and diagnostic agents [3]. These agents might be encapsulated inside the core of NPs or might be adsorbed on the surface individually or in combination. These formulations enhance the pharmacokinetic and pharmacodynamics properties of the NPs by increasing the solubility, dispersion, permeability and overall bioavailability of the formulation. The release of the drug might be controlled passively or actively through various stimuli such as 
temperature, and $\mathrm{pH}$ [4]. All these factors results in the higher concentration of the entrapped drug that reached the systemic circulation that helps to attain the mean effective concentration without producing any toxic effects [5].

Among these DDS, polymeric nanoparticles (NPs), liposomes, niosomes, dendrimers and porous silicon NPs have been extensively employed in the pharmaceutical delivery. Polymeric NPs have versatility in term of their chemical composition and applications. Large type of chemical material based NPs were formulated such as polymeric NPs, Porous silicon NPs, Carbon nanotubes, Graphene NPs and quantum dot. All these DDS have their own pros and cons in term of drug loading, encapsulation, release and applicability [6]. Furthermore, all these DDS were decorated with different chemical reagents and ligands to impart the desired characteristics through modifying the physicochemical properties of the NPs including (1) enhanced means residence time and improved stability, (2) external stimuli driven drug release, (3) controlled and targeted delivery of various chemotherapeutics agents and (4) administration of various theranostic agents [7].

These nanocarriers have been explored due to their extensive potential applications and excellent in vitro performances, but these NPs still have poor in vivo properties in term of their poor solubility in various body fluids, rapid uptake and excretion by the body defense system, poor penetration among the various biological membranes and body tissues, uncontrolled fluctuations in the plasma levels of the active therapeutic components and dose related toxicity issues $[8,9]$.

The most important domains of these nanocarriers include the polymeric DDS and the lipid based vesicular systems. The polymeric DDS provide the variety in term of their structural materials and chemical composition [6]. Different polymers include from the synthetic and natural sources have been employed for the medical applications. These DDS include the polymeric NPs, mesoporous silicon NPs, metal coated NPs, inorganic NPs, dendrimers and the carbon nanotubes [10]. Vesicular DDS include the liposome and noisome. These were defined as the single and the multilayer lipid vesicles while the niosomes were made up of nonionic surfactants instead of the phospholipids [11]. These novel systems provide excellent compatibility with other ingredients, higher and simultaneous encapsulation of the hydrophilic and lyophilic therapeutic moieties and due to lipid nature it provide better pharmacokinetic profiles that might lead to improved therapeutic response of the encapsulated drug. But still this system might suffer from some draw back in term of drug leakage, stability problems and difficulty in the scale up of the process [12].

The above-mentioned problems associated with these DDS including liposomes and polymeric nanoparticles can be overcome by merging their structural components by formulating the lipid-polymer hybrid nanoparticles (LPHNPs). These NPs combine the potential benefits and reduce the different drawbacks of all the individual structural components [13]. These hybrid particles might be produced in different morphologies including the core shell and matrix type LPHNPs. The core or the central material may be encapsulated in single and/or multiple layers of the lipid on the polymeric core materials that also provide the site for the surface modification with different targeting moieties and ligands that help to induce the desired characteristics in the DDS [14].

In this chapter, the different structural components such as lipids and polymers were explained along with the different formulation methods to prepare the LPHNPs along with various process parameters and their pros and cons.

\section{Structural components and their arrangement mechanism}

The core and the shell materials might include different polymeric materials, oils, metal oxides, organic and inorganic compound from the natural and synthetic 
sources that successfully employed for the fabrication of the NPs. These systems have been composed of following major layers and components given as fellows

i. The inner most layer consist of different polymers, organic and inorganic materials that act as a core material. These core materials might be coated with other agents or may form a matrix structure that then functionalized by using the different targeting moieties. These cores of the NPs might encapsulate the hydrophilic or hydrophobic drugs [15].

ii. The second layer of these hybrid NPs is fabricated with the natural or derived lipid that impart the desired pharmacokinetic properties to the DDS. This layer encapsulates the central polymeric core and enhances the compatibility with the biological system. While it also act as a permeability control barrier that limit the release of loaded therapeutic agent as a function of water penetration [16].

iii. The third layer is composed of either lipid or polymer-conjugate that help in the functionalization or surface decoration of the NPs to provide the desired therapeutic of pharmacokinetic effects in term of target specific release and improved retention time of the NPs in the biological system [17].

The mechanism of the arrangement in different layers and their compilation with each other might need further investigation. However, different mechanisms

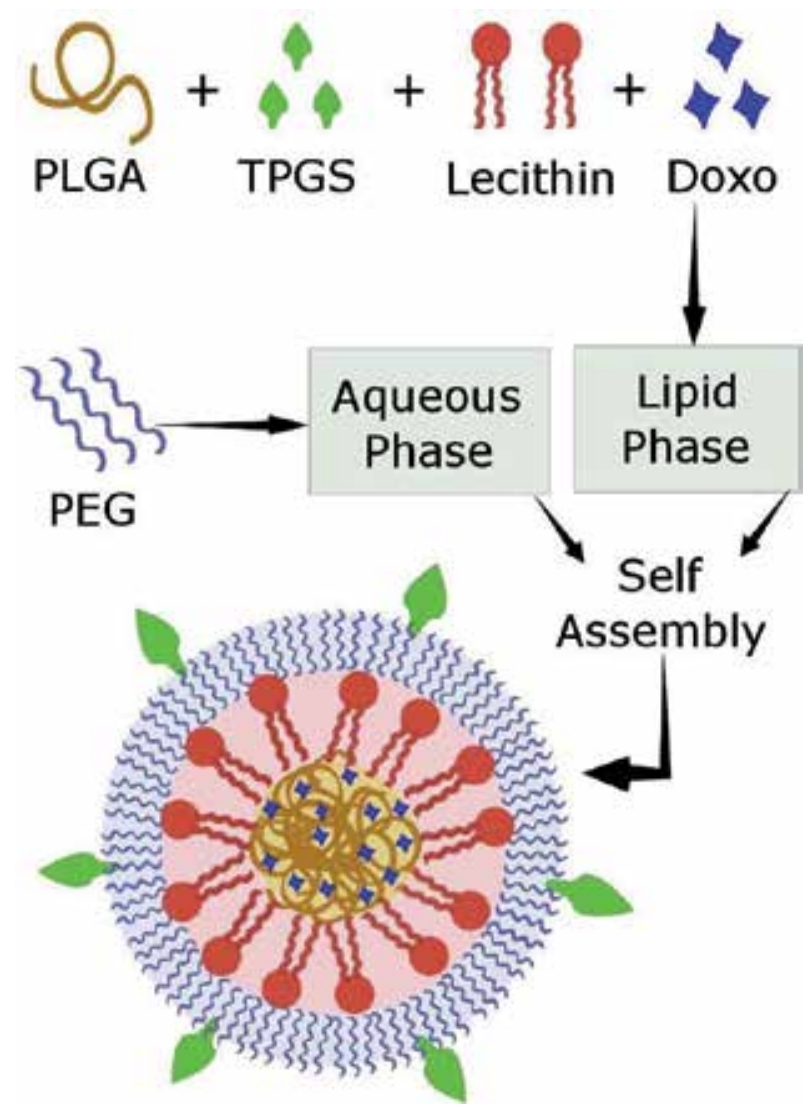

Figure 1.

Schematic diagram of the lipid-polymer hybrid nanoparticles. 
explored indicate that the arrangement and fusion process are based on the method of preparation (that were discussed in the next section). In the two-step conventional method, the layer might be due to formation of lipid by layer that get adhere to the core particle that followed by the integration due to hydrophilic and hydrophobic interaction among the lipid and polymer component. However, in the single-step method, the most investigated and revealed mechanism is the precipitation of the lipid component on the polymeric core material. Some newer techniques might also involve the self-assembling of these structural components (Figure 1) [18].

\section{Method of preparation}

Various formulation methods have been designed and employed for the preparation of LPHNPs based on the chemical and physical nature of the structural components and the desired therapeutic purpose or outcome. These hybrid DDS include the lipid-polymer, lipid metal, polymer-inorganic hybrid, metal (Gold, Silver or Iron) along with polymer hybrid NPs have investigated and employed for the clinical use. Conventionally, two different approaches have been investigated including the two-step and single-step processes. First approach employed the mixing of the inner core and the outer layers to prepare the LPHNPs. While the single-step approach the lipid and polymer that are assembled using the different mechanisms to form the LPHNPs that overcome the drawback of individual components.

\subsection{Two-step conventional method}

Two-step method was the most primitive and frequent method applied for the preparation of different hybrid nanoparticles and other DDS. In the method, the different layers comprise of structurally, different components were separately fabricated and then co incubated to make a complete particle by using the various approaches including the adsorption, self-assembling and encapsulation. The core and shell morphology might be obtained and the various hybrid nanoparticles were obtained by using the sonication [19], solvent emulsification, solvent evaporation [13], nanoprecipitation [20], extrusion, high speed homogenizers and other techniques. However, the selection of the method is based on the physicochemical properties of the loaded drug, size of the core particle and the desired properties that you want to introduce in the NP formulation [18]. For example, the single-step method has been chosen when the encapsulating materials are miscible with the coating substance and soluble in the organic solvent [21].

This method involves multiple preparatory steps to prepare the polymeric core materials and then the lipid vesicles by the different techniques. The polymeric core material might be prepared by dissolving the polymer in a suitable solvent and then precipitated into some nonsolvent phase. Finally, the both components are co-incubated and mixed under gentle stirring for certain time period to allow them to get assembled into lipid-polymer hybrid particles [22, 23]. The mixing may be carried out by vortexing, thin film hydration, probe sonication or extrusion processes so that the final LPHNPs were obtained. These processes actually provide the energy for the mixing, layering or adsorption of the outer coating material on the polymeric core material that might be strengthen by the electrostatic forces among these structural components. 


\subsection{Modified two-step method}

Different modifications have been suggested in the conventional two-step process for the fabrication of the LPHNPs. These modifications might include the use spray drying and lithographic molding along with the freeze drying [24]. The inner central core of the NPs have been prepared with the process that further suspended or dispersed in any suitable organic solvent containing the different structural components of the LPHNPs [2]. Different studies indicate the formulation of LPHNPs loaded with various antibiotics agents including levofloxacin, ciprofloxacin and isoniazid in the form of freeze dried powders for the inhalation therapy that were entrapped in the mono or multiple layers of the lipid shell. The coating of the lipid might provide the core shell morphology to the NPs. This modification in the preparation of the NPs added advantage in term of better inhalation efficiency and greater control on the overall average particle size of the LPHNPs relative to the conventional method [25]. The nanoparticles and hybrid microfiber fabrication are the some other examples that utilized the polyglutamic acid, poly lysine and various grade of PLG and PLA using the freeze-drying method [26, 27].

\subsection{Single-step preparation method}

The poor entrapment and easy leakage of the drug from the polymeric core of the LPHNPs prepared by the conventional two-step methods urged the development of some newer methods that overcome these shortcomings and enhance the therapeutic efficiency of the prepared formulation [14, 28]. The development of single-step method provides the way to control the particle size, PDI, uniformity of the structural components. It also overcomes the variability in different batches and other properties of the LPHNPs in term of their physicochemical properties and stability. The method involves the single preparatory step that considers the mixing of two different phases containing the lipid and polymer in each of the given phase. The mixing process followed by the self-assembling of the structural components that either make the matrix or core shell morphology [29].

Various techniques in the single-step preparation method might include the single or double emulsification method, sonication technique, nanoprecipitation, solvent evaporation method and solvent diffusion method.

\section{Biomedical applications}

LPNs have been prepared for efficient encapsulation and delivery of the wide range of therapeutic agents either alone or in combinations. LPNs have wide range of applications in cancer therapy and delivery of protein based therapeutic agents, i.e., small interfering RNA, nucleic acid and genes delivery etc. Additionally, LPNs can be used for oral drug delivery of many drugs [46]. LPN has wide range of applications in gene and DNA delivery, vaccines and diagnostic imaging agents as shown in Figure 2 [2].

\subsection{Cancer therapy}

Doxorubicin loaded polymer-lipid hybrid nanoparticles (Dox-PLN) were designed and injected intratumorally in mice. At a dose of 0.1 and $0.2 \mathrm{mg}, 70$ and $100 \%$ tumor growth delay was observed, respectively. Dox-PLN treated mice have not shown any sign of toxicity and only 2 mice out of 15 exhibited transient fur 


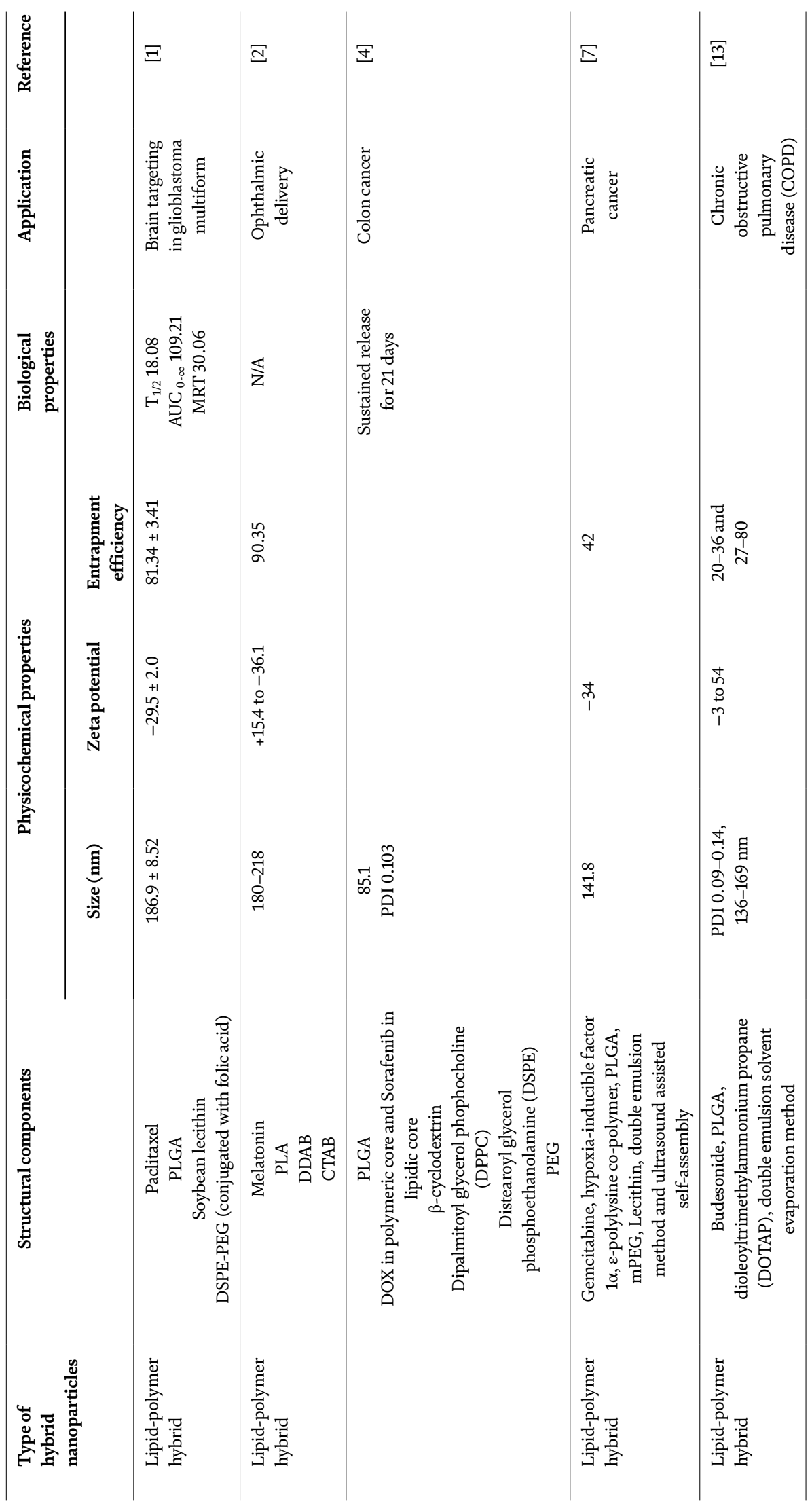


Lipid Polymer Hybrid Nanoparticles: A Novel Approach for Drug Delivery DOI: http://dx.doi.org/10.5772/intechopen.88269

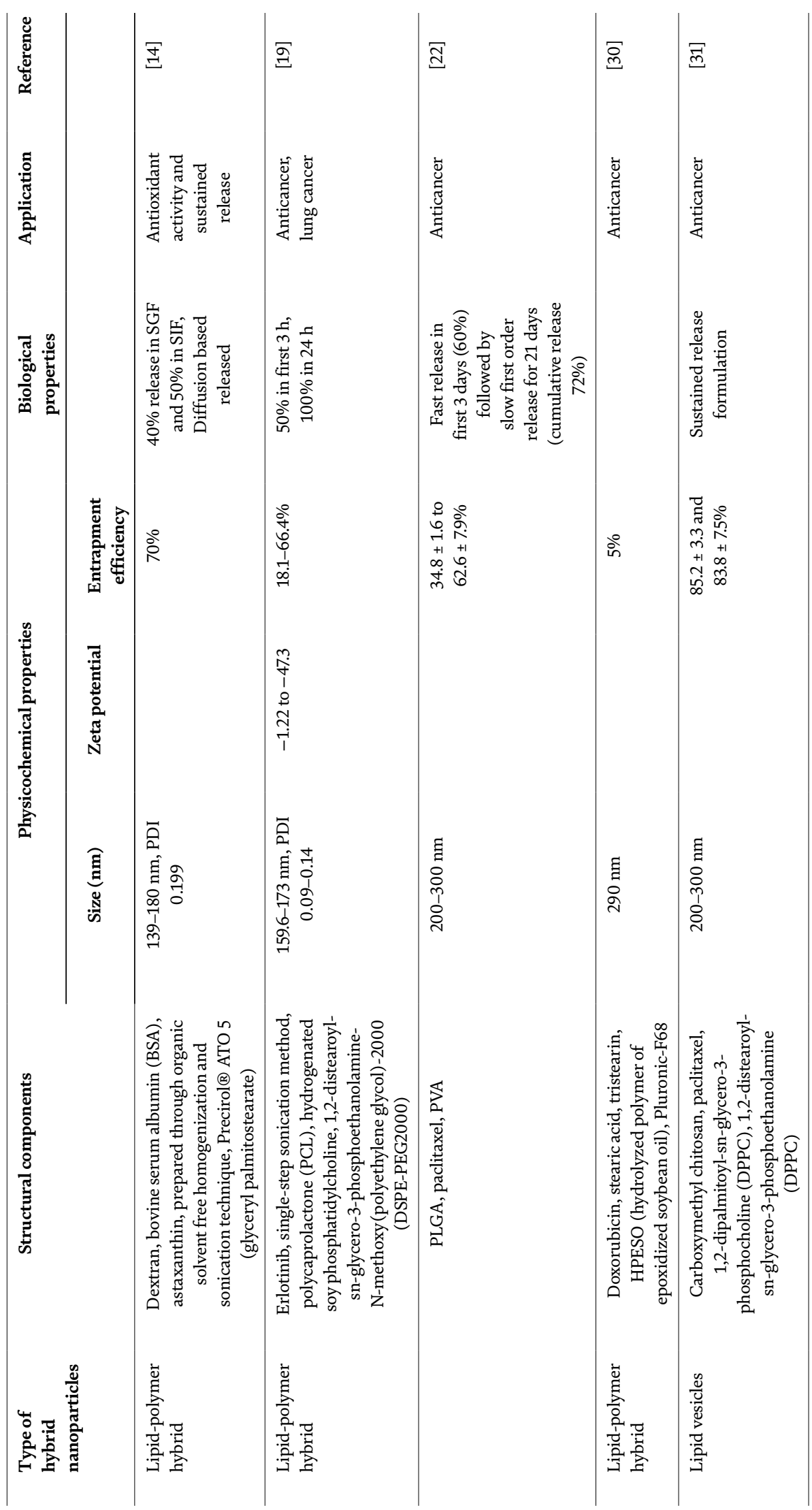




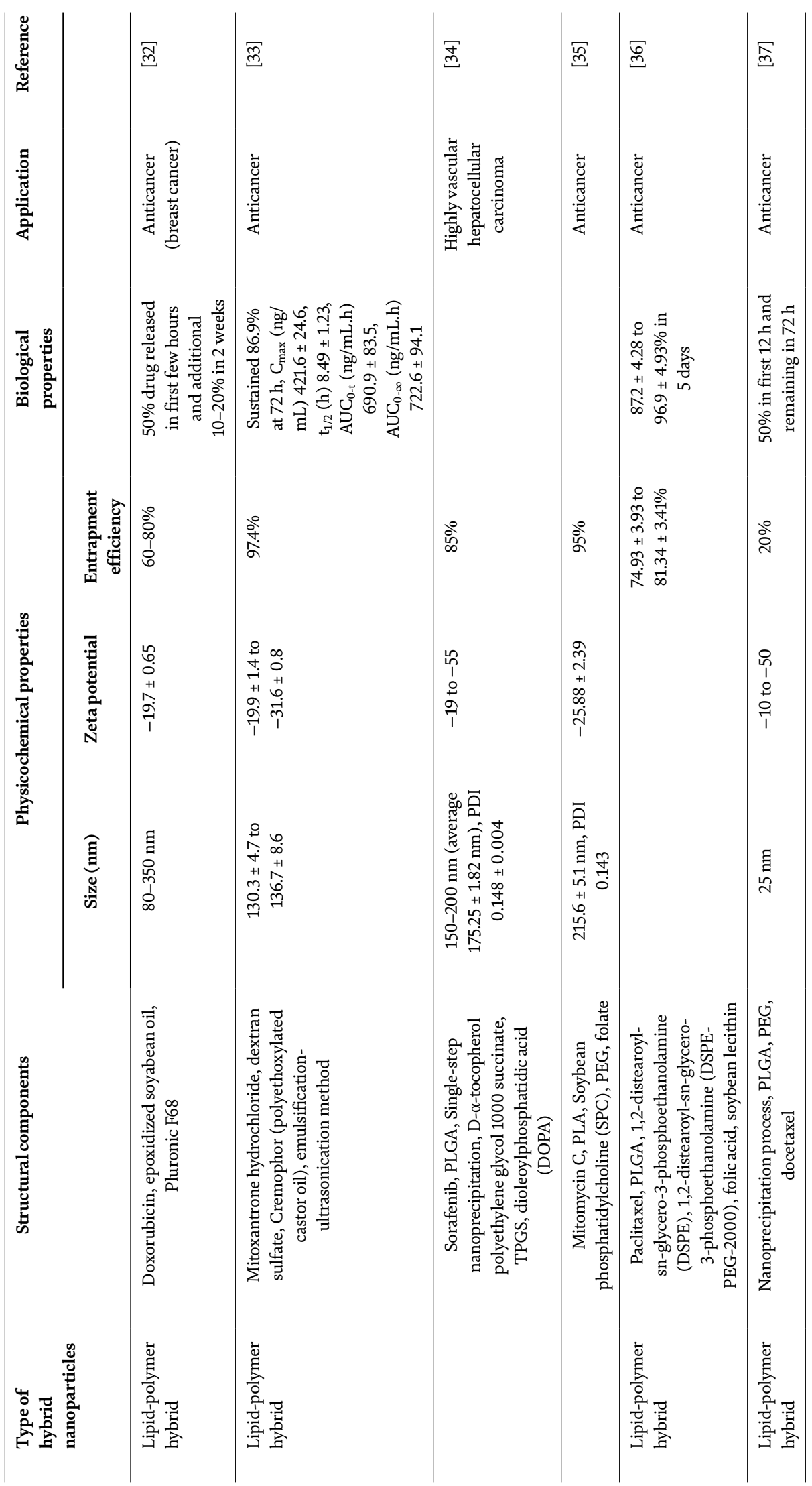


Lipid Polymer Hybrid Nanoparticles: A Novel Approach for Drug Delivery DOI: http://dx.doi.org/10.5772/intechopen.88269

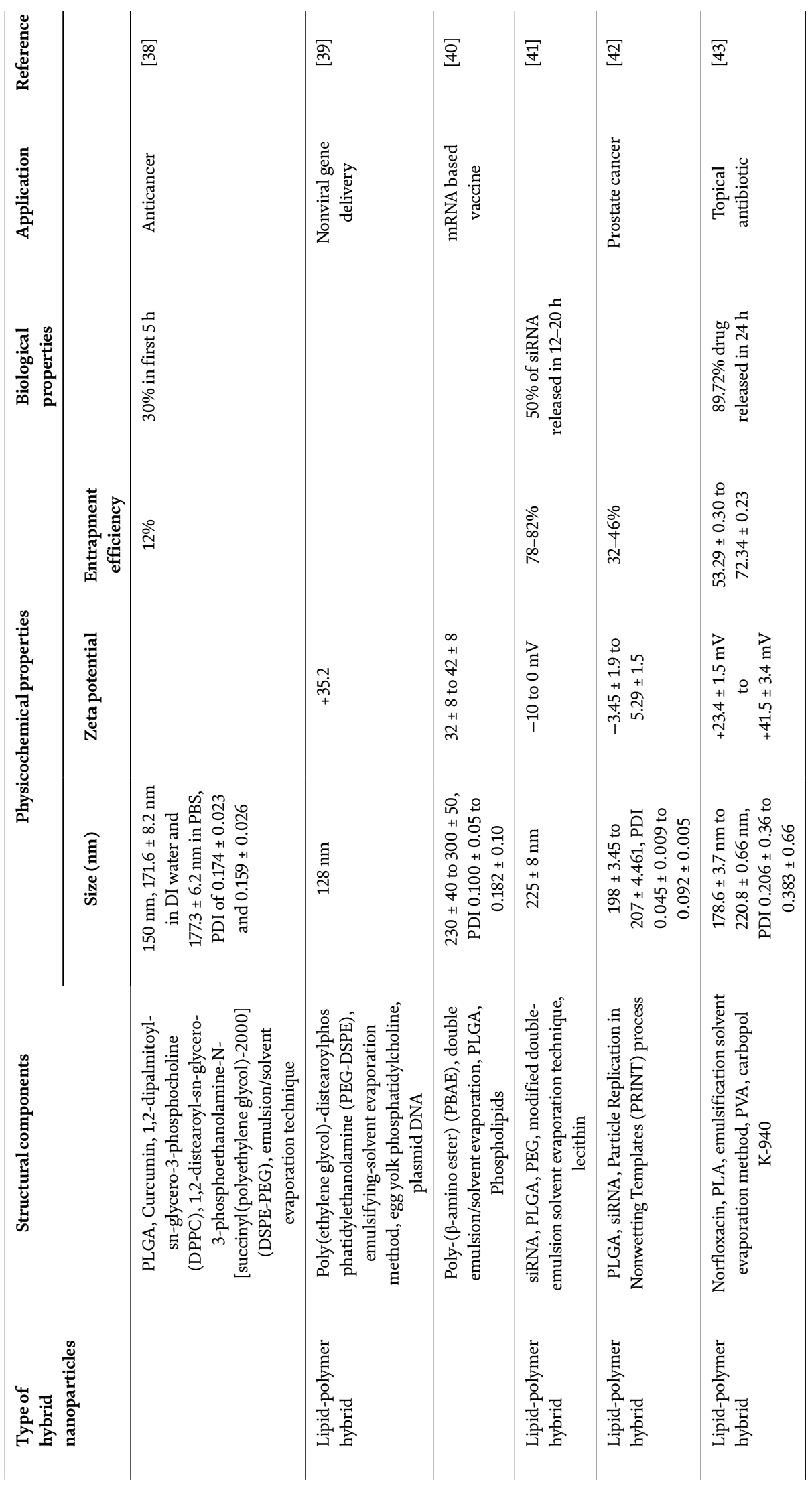




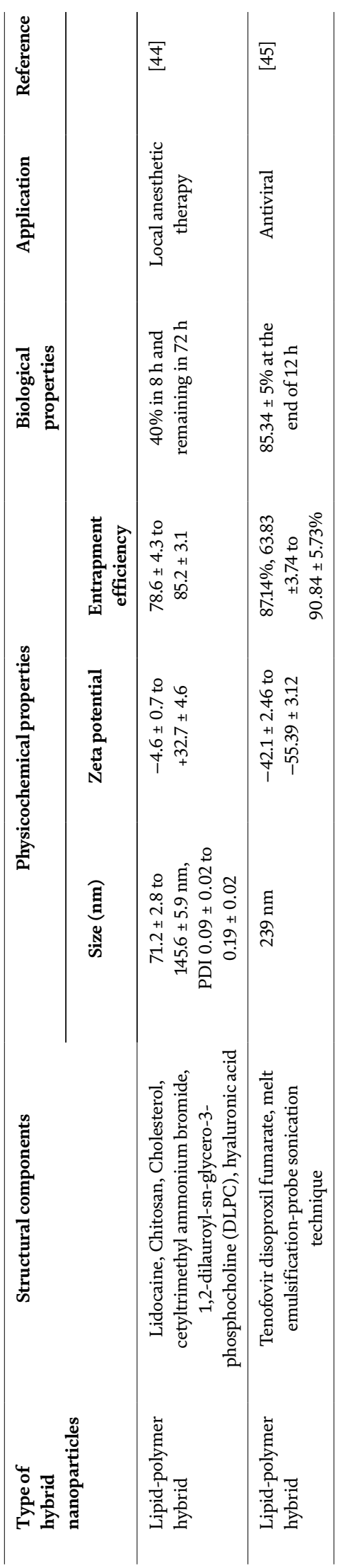




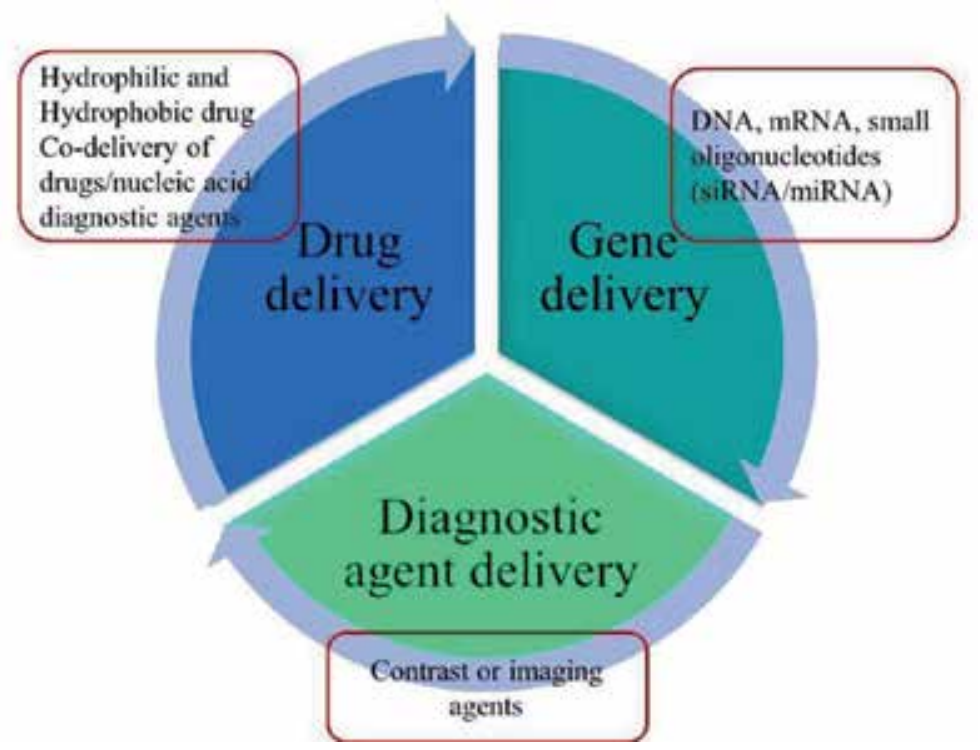

Figure 2.

Applications of lipid-polymer hybrid nanocarriers [46].

roughing. These results indicated that Dox-PLN expressed a good cytotoxic activity against solid tumors and improved the therapeutic efficacy [30].

Paclitaxel loaded LPNs were prepared with a size range of $200-300 \mathrm{~nm}$ for oral administration. Theses LPNs were designed to withstand harsh gastrointestinal tract conditions and improve the bioavailability of paclitaxel. On comparison with Taxol, 1.5- and 5.5-fold increase in bioavailability and elimination half-life was observed, respectively. Additionally, reduction in the reticuloendothelial system mediated uptake by liver and spleen was noted due to stealth characteristics of biopolymer blanket of these LPNs [31].

To overcome the multidrug resistance (MDR) of anticancer drugs, a new strategy was adopted in which doxorubicin loaded solid lipid nanoparticles (SLNs) were complexed with anionic polymer. Due to high encapsulation efficiency (60-80\%) of doxorubicin, the cytotoxicity in tumor cells was increased to 8-fold. Due to physical interaction between drug and polymer and smaller size of nanoparticles (80-350 nm), drug was difficult to clear from target cells by efflux pump [32]. Another strategy to counter MDR is to synthesize lipid-anionic dextran sulfate hybrid carriers loaded with mitoxantrone hydrochloride. The interaction between cationic drug (mitoxantrone hydrochloride) and anionic dextran not only increased drug accumulation but also enhanced the cytotoxicity in breast cancer cell lines. Sustained release of drug (86.9\%) was maintained for $72 \mathrm{~h}$ with an encapsulation efficiency of $97.4 \%$ [33].

Sorafenib is an antiangiogenic agent used in highly vascular hepatocellular carcinoma (HCC). The development of resistance during HCC therapy is mainly due to activation of CXC receptor type 4 (CXCR4). Gao et al. developed PLGA nanoparticles loaded with sorafenib and evaluated the antitumor activity both in vitro and in vivo. On comparing with control group, sorafenib loaded PLGA nanoparticles have shown an improved survival in HCC model, delay in progression of tumor and enhanced antiangiogenic effect [34].

Mitomycin C is a water soluble drug and major disadvantages associated with this drug are poor water stability, rapid elimination and lacking in target specificity. A sustained (up to $120 \mathrm{~h}$ ) and effective delivery of mitomycin C from LPH 
nanoparticles was observed with improved encapsulation efficiency of $95 \%$. Improved cell uptake and site specific accumulation of drug are the major advantages of LPNs [35]. Paclitaxel and folic acid loaded polymer-lipid hybrid nanoparticles were prepared to bypass the tight junctions of blood-brain barrier (BBB) and target the glioma cells. The survival time of mice was increased to 42 days as compared to free paclitaxel which last only 18 days. These targeted nanoparticles have shown better pharmacokinetics and biodistributions which result in better therapeutic outcomes [36].

Ultra-small lipid-polymer hybrid nanoparticles were fabricated using modified nanoprecipitation method. The prepared nanoparticles loaded with docetaxel have the size of $25 \mathrm{~nm}$ which exhibited a better antitumor activity than Taxotere. It was observed that the survival time of Taxotere treated mice were 44 days whereas more than half of the mice treated with ultra-small nanoparticles survived for 64 days. These ultra-small nanoparticles have better biodistribution properties and enhanced permeation ability [37]. Long circulating PLGA nanoparticles loaded with curcumin were fabricated to counter cancer metastasis. The adhesion of cancer cells onto endothelial cells and vascular deposition were reduced by 70 and $50 \%$, respectively. Therefore, these nanoparticles could improve the therapeutic efficacy by preventing metastasis and impairing circulating tumor cells [38].

Core-shell LPN was fabricated to deliver erlotinib using single-step sonication method. In vitro cellular uptake, colony forming assay and luminescent cell viability assay was performed in human lung adenocarcinoma cell line (Figure 3). The mean particle size of LPN is $170 \mathrm{~nm}$ and entrapment efficiency of $66 \%$ with excellent storage stability. The enhanced and efficient uptake of these LPN by cancer cells makes these nanoparticles a potential delivery system for erlotinib [19].

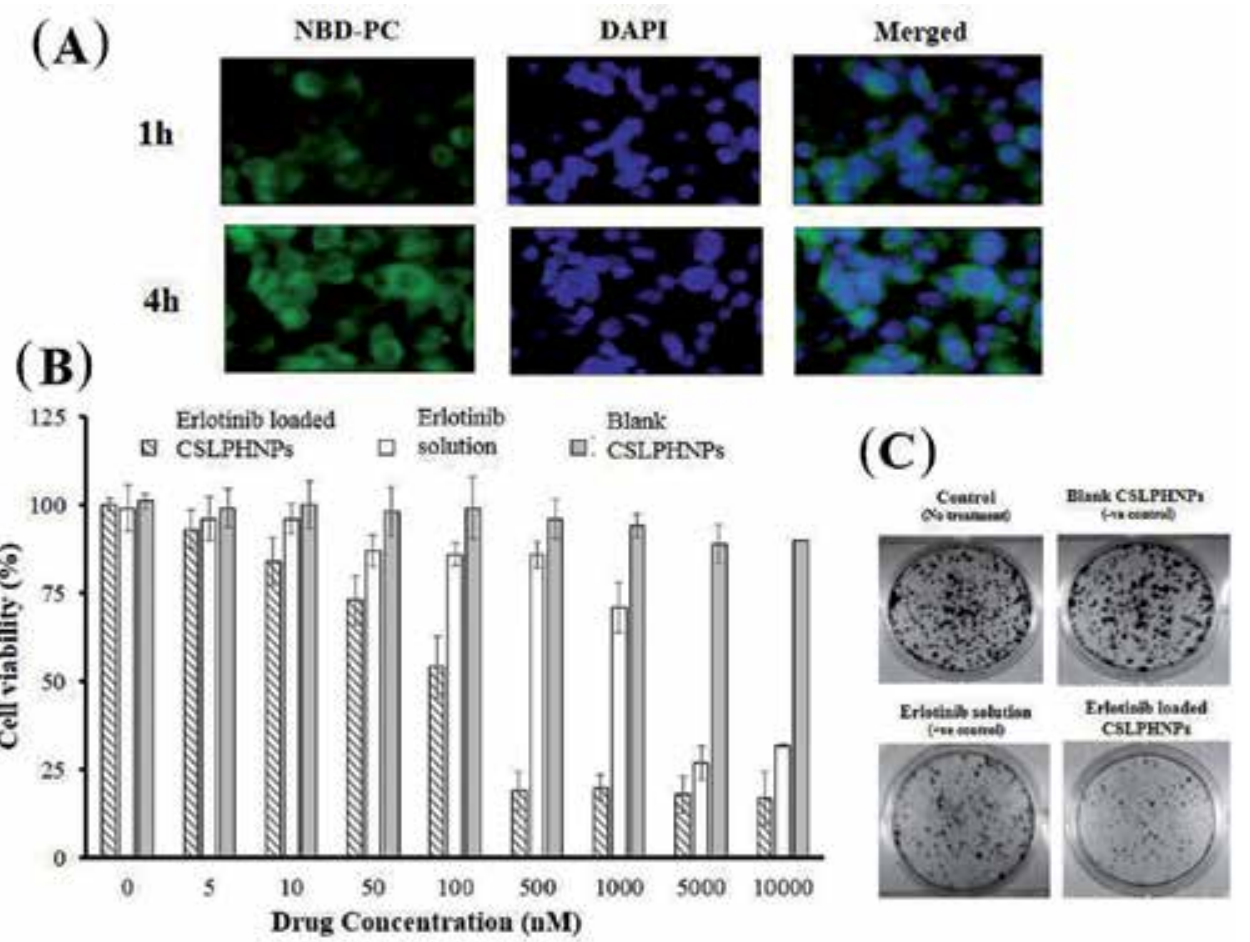

Figure 3 .

(A) Confocal microscopy images of erlotinib loaded CSLPHNPs uptake in A549 cells after 1 and $4 h$, (B) in vitro cellular viability result in $A 549$ cells after $72 h$, and (C) colony formation assay in $A 549$ cells [19]. 


\subsection{Gene delivery}

Plasmid DNA, miRNA and siRNA are now gaining much of the interest of researchers for cancer therapy. Both miRNA and siRNA have different origin and mechanism but similar physicochemical properties. miRNA is endogenous in nature and target the mRNA by developing imperfect pairing and hence act by mRNA degradation, mRNA endonucleolytic cleavage or suppression of translation. siRNA is exogenous in nature and primarily act by endonucleolytic cleavage of target mRNA. siRNA has single mRNA target whereas miRNA has multiple targets. Plasmid DNA carries the recombinant gene or gene of interest and can be administered locally of systemically for cancer therapy [47-50].

Lot of challenges is associated with effective gene delivery especially for cancer therapy. Viral vectors are also facing problems such as development of immunity and inflammatory response, limited carrying ability of DNA and short shelf life [51]. Therefore, the research has now been shifted to nonviral vectors due to nonimmunogenicity, nontoxicity, low cost and feasibility in large scale production. Polyethylene glycol (PEG) and its copolymers have widely used for gene delivery because of its low toxicity, increase water solubility and reduced ability to interact with serum proteins [52].

Effective gene delivery through nonviral vectors with reduced toxicity was developed by emulsification solvent evaporation method. The particle size of newly developed positively charged LPN is in the range from 130 to $240 \mathrm{~nm}$. Fluorescent protein was complexed with plasmid DNA by adsorption and transfection efficiencies was recorded as 37.2 and 34\% for LPN and commercially available product, respectively [39].

Core shell LPN was fabricated using three different methods for incorporation of DNA and the resulted nanoparticles were in the range from 100 to $400 \mathrm{~nm}$. Surface adsorbed DNA, encapsulated DNA and combination of adsorbed and encapsulated DNA are three important methods for fabrication of these nanoparticles. For sustained release of active ingredient, combination method is employed which is necessary for booster vaccination followed by decline release. For primary vaccination (strong and short effective delivery), surface adsorbed mechanism is followed. By adjusting the concentration of different ingredients, the drug release properties can be adjusted [53].

SiRNA delivery through cationic complexes such as polyplexes and lipoplexes has many disadvantages, e.g., development of inflammatory responses, instability and toxicity etc. Small size $(100 \mathrm{~nm})$ with prolong circulation time nanoparticles containing siRNA was developed using PLGA. These hybrid nanoparticles has $80 \%$ encapsulation efficiency of siRNA without any significant degradation until $24 \mathrm{~h}$. Immunofluorescence studies revealed the in vitro apoptosis and $>90 \%$ knockdown of nonsmall cell lung cancer [54].

LPNs are also used for incorporation of mRNA for mRNA vaccines. mRNA was complexed with LPN through electrostatic adsorption to develop 150-300 nm size nanoparticles. These newly developed nanoparticles have shown successful transfection through intranasal route and taken up by dendritic cells with minimum toxicity [40].

A novel approach, modified double emulsion/solvent evaporation method, was used to fabricate hollow core/shell LPNs in which PLGA core was surrounded by lipid shell attached with PEG chains. The size of nanoparticles was $230 \mathrm{~nm}$, $80 \%$ encapsulation efficiency and 50\% siRNA was sustained release for 12-20 h. Moreover, enhanced gene silencing ability was also observed with profound inhibition of gene expression in xenograft tumor [41]. PLGA/siRNA nanoparticles coated with lipids are prepared using Particle Replication in Non wetting Templates technique and exhibited 32-46\% encapsulation efficiency for the treatment of prostate cancer [42]. siRNA was localized in PLGA core at high concentration by varying the 
concentration of polymer and lipid. This localized siRNA then modifies the release, physicochemical properties and transfection efficiencies [55].

\subsection{Theranostic agents}

LPNs not only used for delivering of therapeutic agent but also used for diagnostic purpose. Different type of imaging agents such as quantum dots, fluorescent dyes and iron oxide are incorporated in polymer core. Lipid-polymer and lipid-quantum dot nanoparticles were prepared in a single step with narrow size distribution [56]. Physicochemical properties can be controlled by varying the experimental conditions. Such multicomponent nanoparticles can be used therapy and diagnosis simultaneously.

LPNs are used for theranostic purposes in which phospholipids are attached on one side of polymer chain and fluorophores are linked on the other side. LPNs were designed to incorporate MRI contrast agent, gadolinium, chelated with lipid-PEG in PLGA core. These agents have shown effective uptake of nanoparticles within $3 \mathrm{~h}$ by J-774 cells [57]. Instead of conjugation with imaging agent, fluorescence dye was uploaded in LPNs core surrounded by lipid-PEG shell. The final size of such nanoparticles was in the range from 20 to $30 \mathrm{~nm}$ and bright fluorescence was observed due to lipid tail and polymeric core [58].

\subsection{Stimuli responsive LPNs}

Stimuli responsive drug delivery systems have the ability to deliver therapeutic agents at target sites in a controlled manner with minimum side effects. LPNs comprise of magnetic beads. Stimuli responsive drug delivery system has the advantage to deliver the therapeutic agent in a controlled manner at target site. In a core (PLGA) shell (soyabean lecithin) nanoparticle system, magnetic beads were used for stimuli responsive release of camptothecin when exposed to radio frequency magnetic field. The release of drug was increased by $60 \%$ using radio frequency which significantly decreased the mouse breast cancer cell growth. Such system can be beneficial in cancer chemotherapy due to easy preparation, bio-stability and site specific drug delivery [59].

\subsection{Miscellaneous pharmacological applications}

Hepatitis $\mathrm{C}$ is a chronic disease which leads to liver cirrhosis and hepatocellular carcinoma. LPNs are used to label HCV viral particles for their detection, possible interaction and entrance pathway into host cells [60].

SLNs are developed for topical administration of norfloxacin using solvent evaporation method. These nanoparticles have shown antimicrobial activity against Staphylococcus aureus and Pseudomonas aeruginosa and could be used for the treatment of burn wound and topical infections. SLNs have shown $89.72 \%$ drug release after $24 \mathrm{~h}$ and passed the skin irritation and stability tests [43].

LPNs have recently used for improving the local anesthetic action of lidocaine. Two different kinds of nanocarriers, i.e., liposomes and LPNs were prepared and evaluated the skin permeation ability, in vitro and in vivo drug release studies, encapsulation efficiency and particle size. Results indicated that LPNs has better and improved efficacy of lidocaine as compared to liposomal delivery system due to smaller size $(88.6 \mathrm{~nm})$. The steady state flux of LPNs was found to be $65.4 \mu \mathrm{g} / \mathrm{h} / \mathrm{cm}^{2}$ which showed increased skin permeation capacity [44].

LPNs were used for nasal delivery of an antiviral drug, tenofovir using melt emulsification-probe sonication technique. Intranasal flux of $135.36 \mu \mathrm{g} / \mathrm{cm}^{2} / \mathrm{h}$ and enhanced fluidity improved the drug permeation through membrane phospholipids, which increase the bioavailability of the drug [45]. 


\section{Author details}

Nayab Tahir ${ }^{1 *}$, Muhammad Tahir Haseeb ${ }^{1}$, Asadullah Madni ${ }^{2}$, Farzana Parveen ${ }^{2}$, Muhammad Muzamil Khan ${ }^{2}$, Safiullah Khan ${ }^{2}$, Nasrullah Jan ${ }^{2}$ and Arshad Khan ${ }^{2}$

1 College of Pharmacy, University of Sargodha, Sargodha, Pakistan

2 Department of Pharmacy, The Islamia University of Bahawalpur, Bahawalpur, Pakistan

*Address all correspondence to: nayabtahir132@gmail.com

\section{IntechOpen}

(C) 2019 The Author(s). Licensee IntechOpen. This chapter is distributed under the terms of the Creative Commons Attribution License (http://creativecommons.org/licenses/ by/3.0), which permits unrestricted use, distribution, and reproduction in any medium, provided the original work is properly cited. (cc) BY 


\section{References}

[1] Zhang L et al. Self-assembled lipidpolymer hybrid nanoparticles: A robust drug delivery platform. ACS Nano. 2008;2(8):1696-1702

[2] Hadinoto K, Sundaresan A, Cheow WS. Lipid-polymer hybrid nanoparticles as a new generation therapeutic delivery platform: A review. European Journal of Pharmaceutics and Biopharmaceutics. 2013;85(3):427-443

[3] Vikulina AS, Skirtach AG, Volodkin DV. Hybrids of polymer multilayers, lipids, and nanoparticles. Mimicking the cellular microenvironment. Langmuir. 2019;35(26):8565-8573

[4] Mieszawska AJ et al. Synthesis of polymer-lipid nanoparticles for image-guided delivery of dual modality therapy. Bioconjugate Chemistry. 2013;24(9):1429-1434

[5] Gong J et al. Polymeric micelles drug delivery system in oncology. Journal of Controlled Release. 2012;159(3):312-323

[6] Fan J et al. Targeted anticancer prodrug with mesoporous silica nanoparticles as vehicles. Nanotechnology. 2011;22(45):455102

[7] Zhao X et al. Co-delivery of HIF1 $\alpha$ siRNA and gemcitabine via biocompatible lipid-polymer hybrid nanoparticles for effective treatment of pancreatic cancer. Biomaterials. 2015;46:13-25

[8] Madni A et al. Liposomal drug delivery: A versatile platform for challenging clinical applications. Journal of Pharmacy \& Pharmaceutical Sciences. 2014;17(3):401-426

[9] Mehnert W, Mäder K. Solid lipid nanoparticles: production, characterization and applications. Advanced Drug Delivery Reviews. 2012;64:83-101
[10] Prabaharan M et al. Gold nanoparticles with a monolayer of doxorubicin-conjugated amphiphilic block copolymer for tumor-targeted drug delivery. Biomaterials. 2009;30(30):6065-6075

[11] Khan MI, Madni A, Peltonen L. Development and in-vitro characterization of sorbitan monolaurate and poloxamer 184 based niosomes for oral delivery of diacerein. European Journal of Pharmaceutical Sciences. 2016;95:88-95

[12] Wakaskar RR. General overview of lipid-polymer hybrid nanoparticles, dendrimers, micelles, liposomes, spongosomes and cubosomes. Journal of Drug Targeting. 2018;26(4):311-318

[13] Leng D et al. Engineering of budesonide-loaded lipid-polymer hybrid nanoparticles using a quality-bydesign approach. International Journal of Pharmaceutics. 2018;548(2):740-746

[14] Wang T et al. Solid lipid-polymer hybrid nanoparticles by in situ conjugation for oral delivery of astaxanthin. Journal of Agricultural and Food Chemistry. 2018;66(36):9473-9480

[15] Chaudhary Z et al. Lipid polymer hybrid carrier systems for cancer targeting: A review. International Journal of Polymeric Materials and Polymeric Biomaterials. 2018;67(2):86-100

[16] Pimentel-Moral S et al. Lipid nanocarriers for the loading of polyphenols-A comprehensive review. Advances in Colloid and Interface Science. 2018;260:85-94

[17] Negi JS. Nanolipid materials for drug delivery systems: A comprehensive Review. In: Characterization and Biology of Nanomaterials for Drug Delivery. 2019. pp. 137-163 
[18] Mukherjee A et al. Lipidpolymer hybrid nanoparticles as a next-generation drug delivery platform: State of the art, emerging technologies, and perspectives. International Journal of Nanomedicine. 2019;14:1937

[19] Mandal B et al. Development and in vitro evaluation of core-shell type lipid-polymer hybrid nanoparticles for the delivery of erlotinib in nonsmall cell lung cancer. European Journal of Pharmaceutical Sciences. 2016;81:162-171

[20] Dave V et al. Lipid-polymer hybrid nanoparticles: Synthesis strategies and biomedical applications. Journal of Microbiological Methods. 2019;160:130-142

[21] Chaisri W, Hennink WE, Okonogi S. Preparation and characterization of cephalexin loaded PLGA microspheres. Current Drug Delivery. 2009;6(1):69-75

[22] Dong Y, Feng S-S. Poly (D,Llactide-co-glycolide) (PLGA) nanoparticles prepared by high pressure homogenization for paclitaxel chemotherapy. International Journal of Pharmaceutics. 2007;342(1-2):208-214

[23] Paillard-Giteau A et al. Effect of various additives and polymers on lysozyme release from PLGA microspheres prepared by an s/o/w emulsion technique. European Journal of Pharmaceutics and Biopharmaceutics. 2010;75(2):128-136

[24] Enlow EM, Luft JC, Napier ME, DeSimone JM. Potent engineered PLGA nanoparticles by virtue of exceptionally high chemotherapeutic loadings. Nano Letters. 2011;11(2):808-813

[25] Wang H, Zhao P, Su W, Wang S, Liao Z, Niu R, et al. PLGA/polymeric liposome for targeted drug and gene co-delivery. Biomaterials. 2010;31(33):8741-8748
[26] Hitzman CJ, Elmquist WF, Wattenberg LW, Wiedmann TS. Development of a respirable, sustained release microcarrier for 5-fluorouracil I: In vitro assessment of liposomes, microspheres, and lipid coated nanoparticles. Journal of Pharmaceutical Sciences.

2006;95(5):1114-1126

[27] Keloglu N, Verrier B, Trimaille T, Sohier J. Controlled association and delivery of nanoparticles from jetsprayed hybrid microfibrillar matrices. Colloids and Surfaces. B, Biointerfaces. 2016;140:142-149

[28] Cheow WS, Hadinoto K. Factors affecting drug encapsulation and stability of lipid-polymer hybrid nanoparticles. Colloids and Surfaces. B, Biointerfaces. 2011;85(2):214-220

[29] D'Addio SM, Prud'homme RK. Controlling drug nanoparticle formation by rapid precipitation. Advanced Drug Delivery Reviews. 2011;63(6):417-426

[30] Wong HL et al. In vivo evaluation of a new polymer-lipid hybrid nanoparticle (PLN) formulation of doxorubicin in a murine solid tumor model. European Journal of Pharmaceutics and Biopharmaceutics. 2007;65(3):300-308

[31] Joshi N et al. Carboxymethylchitosan-tethered lipid vesicles: Hybrid nanoblanket for oral delivery of paclitaxel. Biomacromolecules. 2013;14(7):2272-2282

[32] Wong HL et al. A new polymerlipid hybrid nanoparticle system increases cytotoxicity of doxorubicin against multidrug-resistant human breast cancer cells. Pharmaceutical Research. 2006;23(7):1574-1585

[33] Zhang P et al. Novel nanostructured lipid-dextran sulfate hybrid carriers overcome tumor 
multidrug resistance of mitoxantrone hydrochloride. Nanomedicine:

Nanotechnology, Biology and Medicine. 2012;8(2):185-193

[34] Gao D-Y et al. CXCR4-targeted lipid-coated PLGA nanoparticles deliver sorafenib and overcome acquired drug resistance in liver cancer. Biomaterials. 2015;67:194-203

[35] Li Y et al. Mitomycin C-soybean phosphatidylcholine complex-loaded self-assembled PEG-lipid-PLA hybrid nanoparticles for targeted drug delivery and dual-controlled drug release. Molecular Pharmaceutics. 2014;11(8):2915-2927

[36] Agrawal U et al. Tailored polymer-lipid hybrid nanoparticles for the delivery of drug conjugate: Dual strategy for brain targeting. Colloids and Surfaces B: Biointerfaces. 2015;126:414-425

[37] Dehaini D et al. Ultra-small lipid-polymer hybrid nanoparticles for tumor-penetrating drug delivery. Nanoscale. 2016;8(30):14411-14419

[38] Palange AL et al. Lipid-polymer nanoparticles encapsulating curcumin for modulating the vascular deposition of breast cancer cells. Nanomedicine: Nanotechnology, Biology and Medicine. 2014;10(5):e991-e1002

[39] Li J et al. A novel polymer-lipid hybrid nanoparticle for efficient nonviral gene delivery. Acta Pharmacologica Sinica. 2010;31(4):509

[40] $\mathrm{Su} \mathrm{X}$ et al. In vitro and in vivo mRNA delivery using lipid-enveloped $\mathrm{pH}$-responsive polymer nanoparticles. Molecular Pharmaceutics. 2011;8(3):774-787

[41] Shi J et al. Differentially charged hollow core/shell lipid-polymerlipid hybrid nanoparticles for small interfering RNA delivery. Angewandte
Chemie International Edition. 2011;50(31):7027-7031

[42] Hasan W et al. Delivery of multiple siRNAs using lipid-coated PLGA nanoparticles for treatment of prostate cancer. Nano Letters. 2011;12(1):287-292

[43] Dave V et al. Lipid-polymer hybrid nanoparticles: Development \& statistical optimization of norfloxacin for topical drug delivery system. Bioactive Materials. 2017;2(4):269-280

[44] Wang J et al. An alternative choice of lidocaine-loaded liposomes:

Lidocaine-loaded lipid-polymer hybrid nanoparticles for local anesthetic therapy. Drug Delivery. 2016;23(4):1254-1260

[45] Pokharkar VB, Jolly MR, Kumbhar DD. Engineering of a hybrid polymer-lipid nanocarrier for the nasal delivery of tenofovir disoproxil fumarate: Physicochemical, molecular, microstructural, and stability evaluation. European Journal of Pharmaceutical Sciences. 2015;71:99-111

[46] Hallan SS et al. Lipid polymer hybrid as emerging tool in nanocarriers for oral drug delivery. Artificial Cells, Nanomedicine, and Biotechnology. 2016;44(1):334-349

[47] Chitkara D, Mittal A, Mahato RI. miRNAs in pancreatic cancer: Therapeutic potential, delivery challenges and strategies. Advanced Drug Delivery Reviews. 2015;81:34-52

[48] Chitkara D, Singh S, Mittal A. Nanocarrier-based co-delivery of small molecules and siRNA/miRNA for treatment of cancer. Therapeutic Delivery. 2016;7(4):245-255

[49] Husain S et al. Gene therapy for cancer: Regulatory considerations for approval. Cancer Gene Therapy. 2015;22(12):554 
[50] Lam JK et al. siRNA versus miRNA as therapeutics for gene silencing. Molecular Therapy - Nucleic Acids. 2015;4:e252

[51] Oh Y-K, Park TG. siRNA delivery systems for cancer treatment.

Advanced Drug Delivery Reviews. 2009;61(10):850-862

[52] Lee M, Kim SW. Polyethylene glycol-conjugated copolymers for plasmid DNA delivery. Pharmaceutical Research. 2005;22(1):1-10

[53] Zhong Q et al. Optimization of DNA delivery by three classes of hybrid nanoparticle/DNA complexes. Journal of Nanobiotechnology. 2010;8(1):6

[54] Zhu X et al. Long-circulating siRNA nanoparticles for validating Prohibitin1-targeted non-small cell lung cancer treatment. Proceedings of the National Academy of Sciences. 2015;112(25):7779-7784

[55] Colombo S et al. Mechanistic profiling of the siRNA delivery dynamics of lipid-polymer hybrid nanoparticles. Journal of Controlled Release. 2015;201:22-31

[56] Valencia PM et al. Single-step assembly of homogenous lipidpolymeric and lipid-quantum dot nanoparticles enabled by microfluidic rapid mixing. ACS Nano. 2010;4(3):1671-1679

[57] Aryal S et al. Engineered magnetic hybrid nanoparticles with enhanced relaxivity for tumor imaging. Biomaterials. 2013;34(31):7725-7732

[58] Kandel PK et al. Incorporating functionalized polyethylene glycol lipids into reprecipitated conjugated polymer nanoparticles for bioconjugation and targeted labeling of cells. Nanoscale. 2011;3(3):1037-1045
[59] Kong SD et al. Magnetic field activated lipid-polymer hybrid nanoparticles for stimuli-responsive drug release. Acta Biomaterialia. 2013;9(3):5447-5452

[60] Bathfield M et al. Synthesis of lipid- $\alpha$-end-functionalized chains by RAFT polymerization. Stabilization of lipid/polymer particle assemblies. Macromolecules. 2008;41(22):8346-8353 



\title{
Preparation and Characterisation of Niosomal Emulsions as Novel Drug Delivery Vehicle Derived from Natural Seaweeds
}

\author{
Reshma Joy, Franklin John and Jinu George
}

\begin{abstract}
Biomedical field uses polysaccharides for the last one half of the century due to its remarkable applications. Nature is an abundant source for natural polysaccharides made its use widely in this field. In this present chapter study, the potential of alginate-к-carrageenan and alginate-gelatin hydrogel beads for encapsulating curcumin was investigated. The evaluation of different combination of combinations of alginate/ $\kappa$-carrageenan and alginate/gelatin hydrogel beads were developed and drug release properties were compared using curcumin as a model drug. Alginate/ $\kappa$-carrageenan hydrogel beads with 50:50 weight ratio exhibited higher swelling and better drug release percentage than compared to other beads. Antibacterial activity of curcumin released from hydrogel beads against $B$. cereus was established by disc assay. This nanosized vehicles with increases the efficacy and to control the delivery of biologically active substances at their specific site is as important as the drug activity itself.
\end{abstract}

Keywords: $\kappa$-carrageenan, alginate, niosome, hydrogel, drug delivery

\section{Introduction}

Revolution changes and modifies the conservative systems existing in the entire zones of the world. Medicinal chemistry and nanotechnology in that sense practices a revolutionary idea that promises healthy life to every human being. One such important category belongs to drug delivery is the use of natural sources as drug carriers [1]. A number of therapeutic agents show potentiality against cancer, HIV, Tuberculosis, and other kind of diseases, but most of them are not having the potential to overcome the biological barriers inside the body and this causing least effect than actual [2]. This appeal the production of a new system that can able to reduce the typical hitches associated with drugs. We need safe, secure and efficient measures to dose new generation diseases that threatening the life of living beings [3].

Accordingly, among the new discovered diseases, cancer leads as a number one killer disease because of the versatile nature and failure of therapeutic potential against the disease [4]. Recent years witnessed discovery of efficient therapeutic agents but its actual challenge is to overcome the biological barriers inside the body [5]. Most of the drugs are potent to the particular disease but the properties limited 
due to its hydrophobic nature, non-specificity, toxicity to normal cells, less stability, etc. [6]. So the researchers move to deliver these available potent drugs to the pathological site by diminishing all these factors through entrapment in a biocompatible polymers as carriers [7]. They were trying to develop smart drug carriers to enhance the efficiency of drugs at its specific site with controlled delivery [8].

Drug delivery is a term that refers the successful delivery of therapeutic agents at the specific site with specific time without any kind of degradation [9]. The problem associated with available drugs in the market includes hydrophobic nature, higher toxic nature, nonspecific release, in vivo degradation and short circulating half-lives [10]. Drug delivery systems emerging from the significance of controlled release of therapeutic agent on the affected part in the effective time with least side effects $[1,11]$. Natural polymer based drug carriers is the central focus due to biocompatible nature, nontoxicity, low cost, ease of use and biodegradability [10]. Specificity [12] of drug molecule can be improved using ligands at the surface of delivery systems which in turn termed targeted drug delivery to the affected area of the body [13]. Targeting can be through either passive or active [14]. Major types of drug carriers falling under

- Niosomes,

- Liposomes,

- Polymeric micelles,

- Microspheres,

- Nanostructures,

- Nanofibers,

- Protein-DNA complexes,

- Protein-drug conjugates,

- Erythrocytes,

- Virosomes,

- Dendrimers.

Utilization of drug delivery systems is a promising methodology for creating efficient therapeutic agents. Among these kinds of carrier's polymeric micelles, dendrimers, liposomes [15] are the growing area because of their outstanding properties and smart drug delivery potential [16]. Polymer based systems must meet some important features to become a good delivery vehicle such as; the backbone should be biocompatible; the term biocompatible in the sense explains capacity of a polymer to act with proper host reaction as well as the polymers are biodegradable without the formation of any kind of harmful by-products inside the body [17-19]. The polymer must soluble in various solvents and versatile in chemical, structural and in application, etc. [3, 9]. The exploitation of micelles prepared from amphiphilic copolymers for solubilization of ineffectively solvent medications has drawn in much consideration. Natural polysaccharide gets much attention towards the preparation of drug delivery systems because of their exceptional 

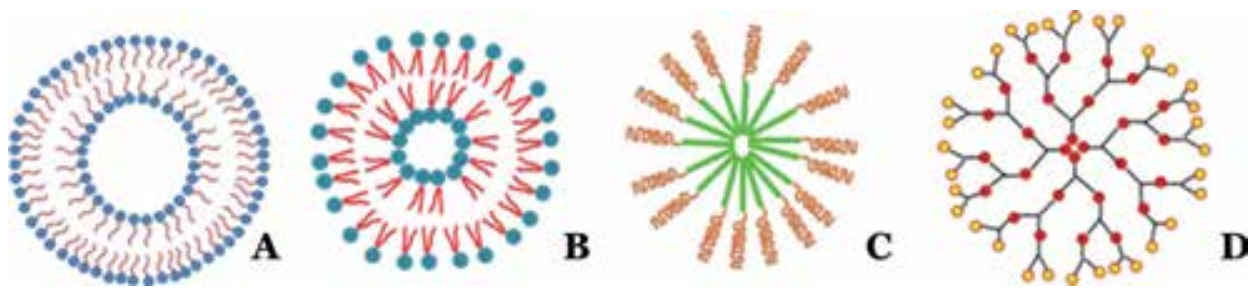

Figure 1.

(A) Niosome, (B) liposome, (C) polymeric micelles and (D) dendrimers.

hydrogel forming ability [20]. These are derived from plants, sea weeds shells of crustaceans and microorganisms [21].

Niosomes are one of the excellent drug carriers, composed of non-ionic surface in addition to cholesterol or its derivatives. Niosomes can overcome the disadvantages of the liposomes [22, 23]. Liposomes firstly proposed by Alec Bangham called Bangosomes in 1965, the first generation liposomes the entrapped drugs are leaked out because of the membranes are not much stronger. In the case of niosomes, the hydrophilic and hydrophobic parts influence the entrapment of drug molecules, where as in the liposomes, lipophilic domain influences in it [5, 24, 25]. Non-ionic surfactants Tween, Brij, Span, etc. are mostly used for the noisome preparation. Hydrophilic-lipophilic balance (HLB) is a dimensionless parameter which can direct the entrapment efficiency for the drug and controlled release [26]. These values are varies between the non-ionic surfactant nature [27-30]. Surfactants with a HLB number between 3 and 8 are compatible with preparation bilayer surfaces and refer to water-in-oil (W/O) emulsifier $[2,31]$. The additives in the niosomes include cholesterol and charge inducer. The cholesterol reduces the HLB value and charge inducer like dicetyl phosphate (DCP), produce surface charge leads to the stabilization of the bilayer $[32,33]$. Applications of niosomes mainly related to the systemic delivery of hydrophobic drug molecules. More clearly niosome is used to improve the stability and physical properties, controlled release of drug molecules and for targeting and retention of drug in blood circulation. Niosomes offer various advantages over other drug delivery devices and have found applicability in pharmaceutical field. Niosomes are exceptionally powerful drug delivery instruments for construction/focusing of different remedially dynamic moieties and the responsibility lies on future researchers to viably outfit its potential in assorted application zones to support humanity [34].

Hydrophilic interpenetrating polymeric networks like hydrogels represent excellent carrier for hydrophobic drug encapsulation. In comparison to hydrophilic nano systems widely used in drug delivery, hydrogels show better swelling property and biocompatibility $[35,36]$. Hydrogels derived from natural polymers like polysaccharides, alginates, gelatin polysaccharide conjugates exhibit a gradual change in swelling properties upon variation in temperature, $\mathrm{pH}$, and ionic strength. 3D polymeric network derived from hydrogels represent flexible tissue-like material for efficient delivery of hydrophobic drugs [37] (Figure 1).

\section{Natural polysaccharides}

The use of nanotechnology for the delivery of therapeutic agents was put forward by Paul Enrich [6], the proponent of drug loaded magic bullets. Biomedical field uses polysaccharides for the last one half of the century due to its remarkable applications [38]. Nature is an abundant source for natural polysaccharides made its use widely in this field. Most of the drugs used for different types of diseases 
cause dangerous side effects. These problems can be reduced by the use of natural biocompatible polymer/polysaccharides as carriers for the drug. Seaweeds provide a better source for available polysaccharides such as carrageenan (iota, kappa, and lambda), alginate, and agar [39].

Monosaccharides combine to form polysaccharides with number of reactive groups that can be easily modified to get various types of polysaccharides. Typical sources of polysaccharide include algae, microorganisms, animals, etc. The positive features of polysaccharides such as higher molecular weight, changing chemical composition and large number of reactive groups gave diversity in its function. Adaptable chemical composition delivers its variety in structure and also in property. Medicinal field exploits these best properties of polysaccharides for the betterment of drug delivery. Drug carriers from polysaccharides make sustainable, cost effective and biodegradable drug carriers. Modification of polysaccharide chains opens a way to target drug to the affected site, altering the solubility, biocompatibility, moisture resistant and particularly reduce the severe side effects from therapeutic agents. Polysaccharides can perform bio adhesion through hydrophilic groups such as carboxyl, hydroxyl and amino groups by making non covalent interaction with biological tissues that elongates residing time. By this the absorption of drug at the particular area can be easily made. In this chapter we discuss niosomal emulsions of alginate, $\kappa$-carrageenan and gelatin as drug carrier [38, 40, 41].

Alginates are naturally derived polysaccharide block copolymers composed of $\beta$-D-mannuronic acid monomers (M-blocks), $\alpha$-L-guluronic acids (G-blocks) and regions of interspersed $M$ and $\mathrm{G}$ units $[38,42]$. Divalent cations such as $\mathrm{Ca}^{2+}$ can induce hydrogel formation by polysaccharide chain association in alginates [20]. Carrageenans represent a family of sulphated linear polysaccharides consisting of $(1 \rightarrow 3)$-linked $\beta$-galactose and $(1 \rightarrow 4)$-linked $\alpha$-D-galactose units mainly extracted from certain red seaweeds of Rhodophyceae family particularly from Chondrus crispus, Eucheuma gigartina, Stellate iridaea, Hypnea, Solieria agardhiella and Sarconema, which are variously substituted and modified to the 3,6-anhydro derivative, depending on the nature of origin and extraction conditions [43]. Three main types of carrageenans are known: kappa- $\kappa$, lambda- $\lambda$ and iota- $i$, depending on the number and the position of the ionic sulphate groups. The presence of a suitable cation, typically potassium, or calcium is an absolute requirement for gelation of the carrageenans, especially kappa [44]. Gelatin is a protein derived from denatured collagen that contains high levels of hydroxyproline, proline and glycine. It is useful as a thermally reversible gelling agent for encapsulation [45].

However, we go through the development and characterization of alginate/ $\kappa$ carrageenan and alginate/gelatin hydrogel beads and its application in encapsulation of a model hydrophobic potential drug, curcumin. Hydrogel beads were developed via ionotropic gelation. Alginate/ $\kappa$-carrageenan beads were cross-linked with $\mathrm{CaCl}_{2}-\mathrm{KCl}$ salt solution and alginate/gelatin beads with $\mathrm{CaCl}_{2}$ solution. Drug release behaviour of alginate/ $\kappa$-carrageenan and alginate/gelatin hydrogel beads was compared employing curcumin, which is a natural polyphenolic compound isolated from rhizome of turmeric (Curcuma longa) as a model drug [46]. Curcumin is widely known to possess antioxidant [47], antitumor [48], and anti-inflammatory [49] activity. Swelling of the beads and in vitro release of curcumin were studied in phosphate buffered saline (PBS, $\mathrm{pH} 7.4$ ).

\section{Materials and methods}

Sodium alginate (RM 7494), $\kappa$-carrageenan (22,048, Sigma Aldrich), Gelatin, phosphate buffer saline pH 7.4 (PBS, Sigma Aldrich) and curcumin (C1386, Sigma 
Preparation and Characterisation of Niosomal Emulsions as Novel Drug Delivery Vehicle... DOI: http://dx.doi.org/10.5772/intechopen.86942

\begin{tabular}{lcccc}
\hline Materials & $\begin{array}{c}\text { Weight } \\
\text { ratio }(\mathbf{w} / \mathbf{w})\end{array}$ & $\begin{array}{c}\text { Polymeric } \\
\text { concentrations }(\%)\end{array}$ & Salt type & $\begin{array}{c}\text { Salt concentration } \\
(\%)(\mathbf{w} / \mathbf{v})\end{array}$ \\
\hline Alginate/ $\boldsymbol{k}$-carrageenan & $50: 50$ & 1 & $\mathrm{CaCl}_{2} \cdot \mathrm{KCl}$ & 2 \\
& $70: 30$ & 1.5 & & \\
& $80: 20$ & 2 & & \\
\hline Alginate/gelatin & $50: 50$ & 1 & $\mathrm{CaCl}_{2}$ & 2 \\
& $70: 30$ & 1.5 & & \\
& $80: 20$ & 2 & & \\
& & 2.5 & & \\
\hline
\end{tabular}

Table 1.

Weight ratio percentage concentrations of polymeric solutions.

Aldrich) were used without further purification. $\mathrm{CaCl}_{2}$ and $\mathrm{KCl}$ were commercial products of analytical grade. Single distilled water (Labsil Water Distiller, Model: OPTI-M4) was used in all experiments.

\subsection{Preparation of alginate/к-carrageenan and alginate/gelatin hydrogel beads and curcumin loading}

Alginate, $\kappa$-carrageenan and gelatin solutions were prepared separately by dissolving each of the biopolymers in distilled water and heated up at $50^{\circ} \mathrm{C}$ (for alginate), $60^{\circ} \mathrm{C}$ (for $\kappa$-carrageenan) and $40^{\circ} \mathrm{C}$ (for gelatin) under constant stirring from $30 \mathrm{~min}$ to $1 \mathrm{~h}$ until complete dissolution [50]. Ionotropic method was used for the preparation of hydrogel beads. Polysaccharide mixture composed of 50:50, 70:30, 80:20 (weight ratio) with concentrations varying from 1 to $2.5 \%$ (given in Table 1) were prepared by mixing under constant stirring at $30^{\circ} \mathrm{C}$ for $30 \mathrm{~min}$. Briefly the hydrogel beads were prepared by dropping the mixture through a plastic syringe $(5 \mathrm{ml})$ into an aqueous salt solutions stirred

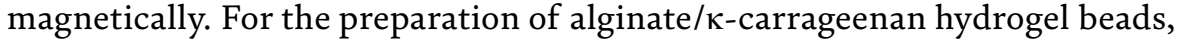
the salt solution was composed of $100 \mathrm{ml}$ of $2 \%$ (w/v) $\mathrm{KCl}$ and $100 \mathrm{ml}$ of $2 \%$ $(\mathrm{w} / \mathrm{v}) \mathrm{CaCl}_{2}$ and for alginate/gelatin hydrogel beads, $200 \mathrm{ml}$ of $2 \% \mathrm{CaCl}_{2}$ solution. To complete gelation, beads were maintained in the solution for $30 \mathrm{~min}$ then filtered, followed by washing with distilled water and then allowed to dry overnight at $37^{\circ} \mathrm{C}$.

For curcumin loading, $10 \mathrm{mg} / \mathrm{ml}$ curcumin prepared with ethanol was mixed with each of the alginate/ $\kappa$-carrageenan and alginate/gelatin mixtures and stirred until complete evaporation of ethanol. Curcumin loaded beads were prepared by dropping the mixture through a $5 \mathrm{ml}$ syringe into a stirred salt solution which was composed of $100 \mathrm{ml}$ of $2 \%(\mathrm{w} / \mathrm{v}) \mathrm{KCl}$ and $100 \mathrm{ml}$ of $2 \%(\mathrm{w} / \mathrm{v}) \mathrm{CaCl}_{2}$ in the case of alginate/ $\mathrm{\kappa}$-carrageenan and $200 \mathrm{ml}$ of $2 \%(\mathrm{w} / \mathrm{v}) \mathrm{CaCl}_{2}$ for alginate/gelatin and kept in the solution for $30 \mathrm{~min}$, filtered, washed with distilled water and dried overnight at $37^{\circ} \mathrm{C}$.

\subsection{Morphological analysis}

Scanning electron microscopy (SEM, LEO $1430 \mathrm{~V}$ ) was used to analyse the morphology of hydrogel beads.

\subsection{Swelling behaviour}

The swelling degree (SD) was determined gravimetrically as follows: Both the hydrogel beads were immersed in phosphate buffered saline (PBS, pH 7.4) at $37^{\circ}$ 
C. The swelled samples were taken from the PBS at selected time intervals of 1, 2, 3, 4 , and 10 up to $24 \mathrm{~h}$, wiped with tissue paper, weighed and placed again in PBS. The $\mathrm{SD}$, in percentage, was calculated using Eq. (1)

$$
\mathrm{SD}=\frac{\mathrm{Ws}-\mathrm{Wd}}{\mathrm{Wd}} * 100(\%)
$$

where Ws and Wd are the weights of swollen and dry beads, respectively.

\subsection{Release study of curcumin}

The cumulative release of curcumin from hydrogel beads was carried out through incubating samples while shaking at $100 \mathrm{rpm}$ in $20 \mathrm{ml}$ of buffer solution (PBS) at $\mathrm{pH} 7.4\left(1,2,3,4,10\right.$ up to $24 \mathrm{~h}$ incubation) at $37^{\circ} \mathrm{C}$. After incubation, $3 \mathrm{ml}$ sample was taken out from the buffer solution and analysed by UV-Vis spectrophotometer (Lambda 25-PerkinElmer UV-vis spectrophotometer) at $\lambda_{\max }$ of $430 \mathrm{~nm}$. The withdrawn volume of the sample was replaced with equal volume of fresh buffer solution to keep the volume of release fluid constant. The percentage drug release was calculated by using the following formula,

$$
\begin{aligned}
\text { Percentage drug release }= & (\text { Amount of drug released } / \\
& \text { Amount of drug loaded }) * 100
\end{aligned}
$$

\section{Results and discussion}

\subsection{Morphological analysis of hydrogel beads}

Image of the swollen beads is shown in Figures 2 and 3. Beads obtained has diameter of $0.50 \mathrm{~cm}$. Morphological features of curcumin loaded and unloaded beads were analysed through SEM images shown in Figure 4.

The morphological features of curcumin loaded and unloaded beads were analysed by recording the SEM images as shown in Figure 4.

Hydrogel beads containing alginate were smoother and more spherical compared to the beads consisting of alginate and carrageenan. This effect can be attributed to the higher cross linking ability of alginate relative to carrageenan. This cross linking takes place instantaneously upon dropping sodium alginate into the cross linking solution (consisting of $\mathrm{Ca}^{2+}$ ) leading to geometrically stable particles.

In the case of formulations containing carrageenan, the beads were less spherical, having rough and folded surfaces. This behaviour could be attributed to the low concentration of $\mathrm{K}^{+}$in the hardening solution, and also to the reduced cross-linking efficiency of carrageenan as compared to alginate, which led to the production of non-spherical and less physically stable beads. The alginate-gelatin hydrogel beads show a smooth and homogeneous morphology, suggesting component miscibility and blend homogeny.

\subsection{Swelling degree}

\subsubsection{Effect of alginate, $\kappa$-carrageenan and gelatin ratios on swelling}

In the case of alginate-gelatin and alginate/ $\kappa$-carrageenan hydrogel beads, the effect of sodium alginate on swelling of the hydrogel beads has been studied by varying its amount in the polymer blend in the range $50-80 \%(\mathrm{w} / \mathrm{w})$. The results shown in Figures 5 and 6, clearly indicates that initially at $50 \%$ of alginate 
Preparation and Characterisation of Niosomal Emulsions as Novel Drug Delivery Vehicle... DOI: http://dx.doi.org/10.5772/intechopen.86942

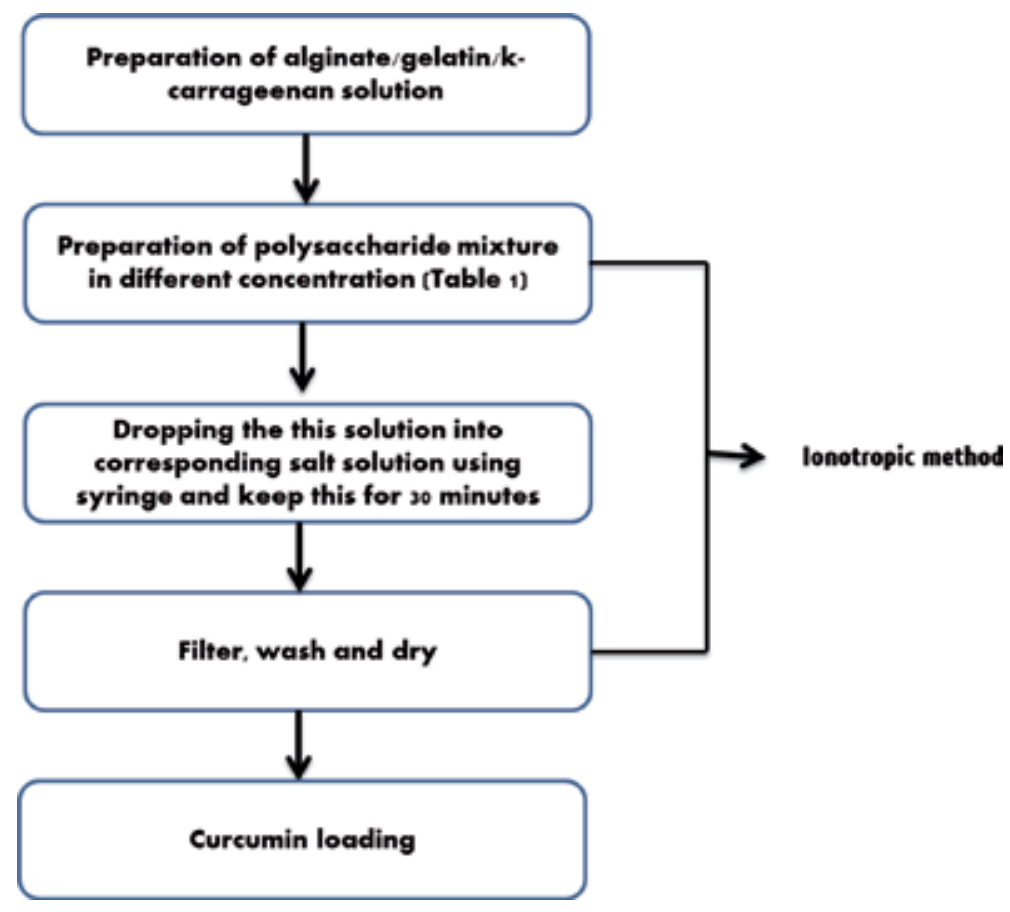

Figure 2.

Preparation of hydrogel beads and drug loading.

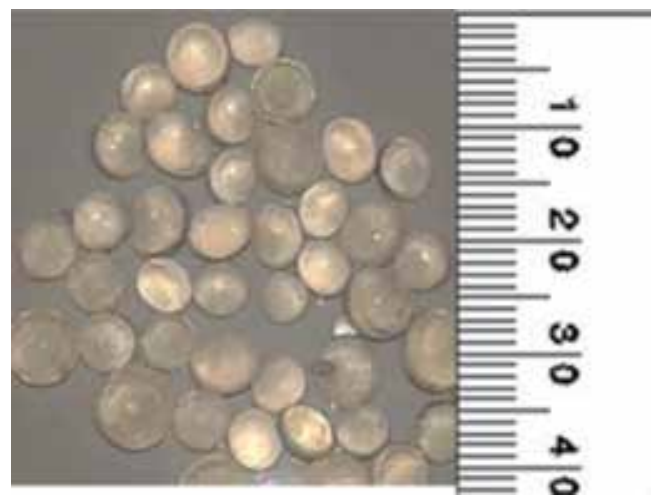

Figure 3.

Image of the swollen beads.

content in the hydrogel beads, the swelling ratio is high, while beyond this amount (70-80\%) a fall in swelling degree is observed. The observed initial increase in the swelling degree could be attributed to the fact that since alginate is a linear anionic hydrophilic polymer, its increasing amount in the hydrogel beads cause an increased hydrophilicity of the polymer network with fixed ionic charges and enhanced repulsion among the polymer chains. This leads to greater swelling. However the decrease in swelling degree observed beyond $50 \%$ can be attributed to the fact that increase in alginate content results in a compact polymer network. This will form small pore sizes that slow down the diffusion of water molecules into the hydrogel beads and consequently the swelling degree decreases.

The effect of gelatin on the swelling degree of the alginate/gelatin hydrogel beads has been studied by varying the amount of gelatin in the polymer blend in the range $20-50 \%(w / w)$. Figure 5 clearly indicates that, an increase in swelling 

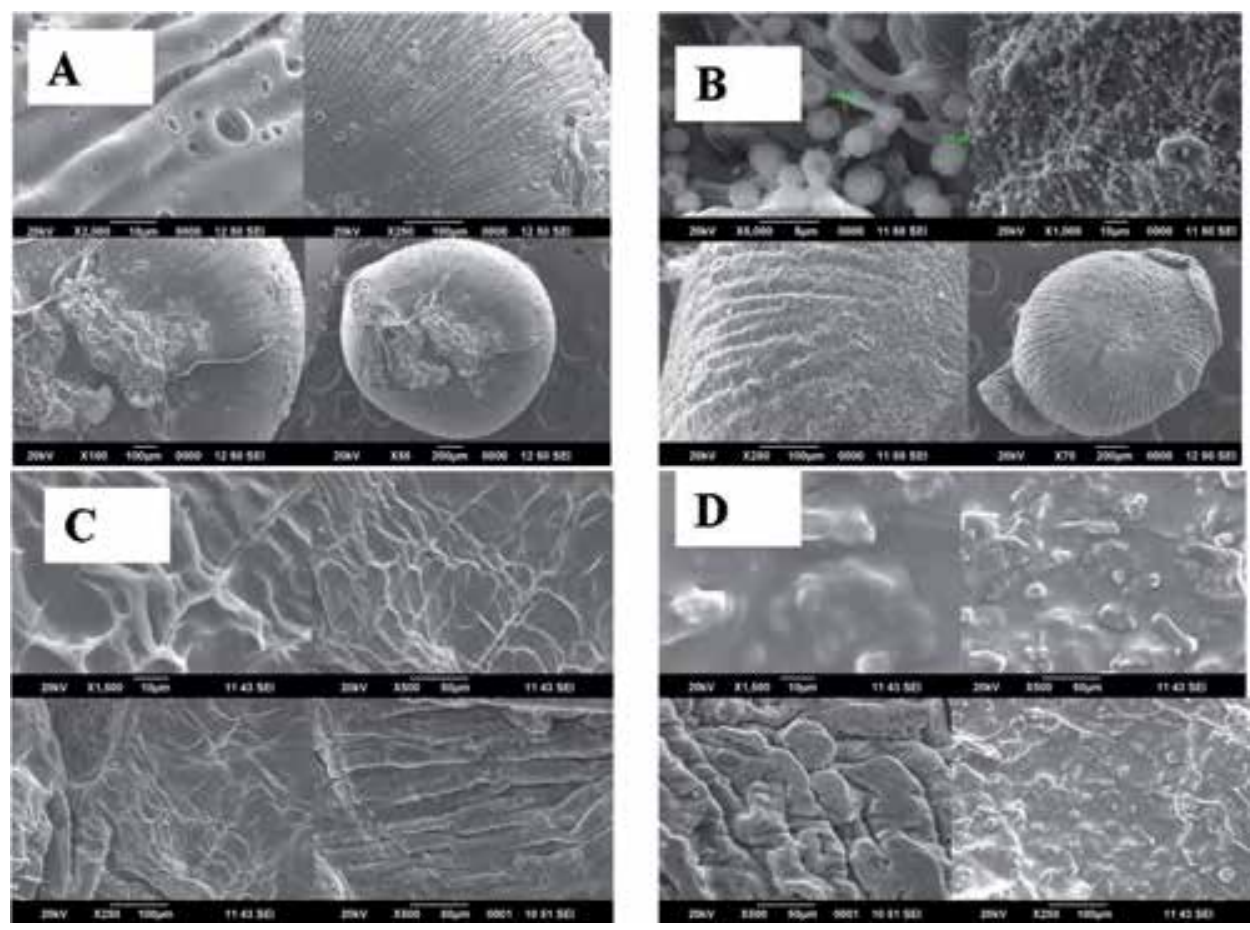

Figure 4 .

SEM micrographs of unloaded $(A)$ alginate/ $\kappa$-Car, (B) alginate/gelatin hydrogel beads and curcumin loaded, (C) alginate/ $\kappa$-Car, (D) alginate/gelatin hydrogel beads.
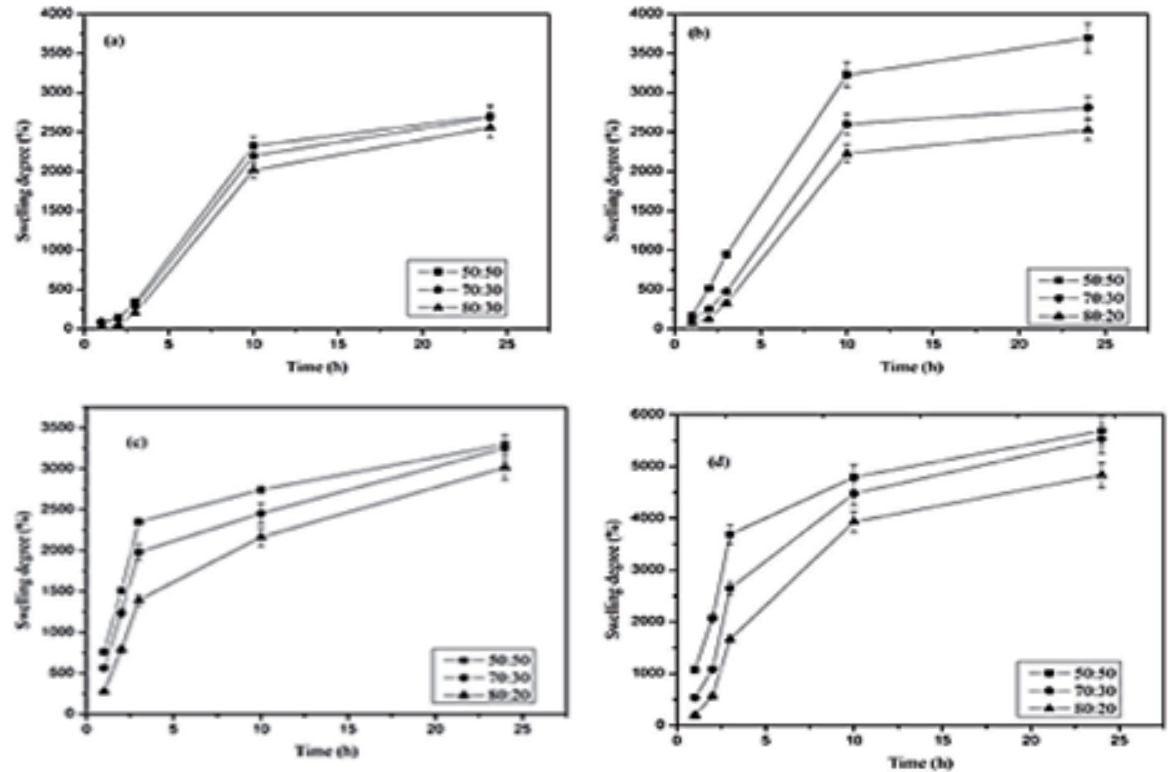

Figure 5.

Effect of alginate/gelatin weight ratio's on the swelling of the alginate/gelatin composite hydrogel beads at different polymer concentrations (a) $1 \%$, (b) $1.5 \%$, (c) $2 \%$, (d) $2.5 \%$.

degree is observed with increase in gelatin content. An increase in the amount of gelatin in hydrogel beads enhances the hydrophilicity of polymer network and leads to higher swelling degree. The effect of $\kappa$-carrageenan on swelling degree of 

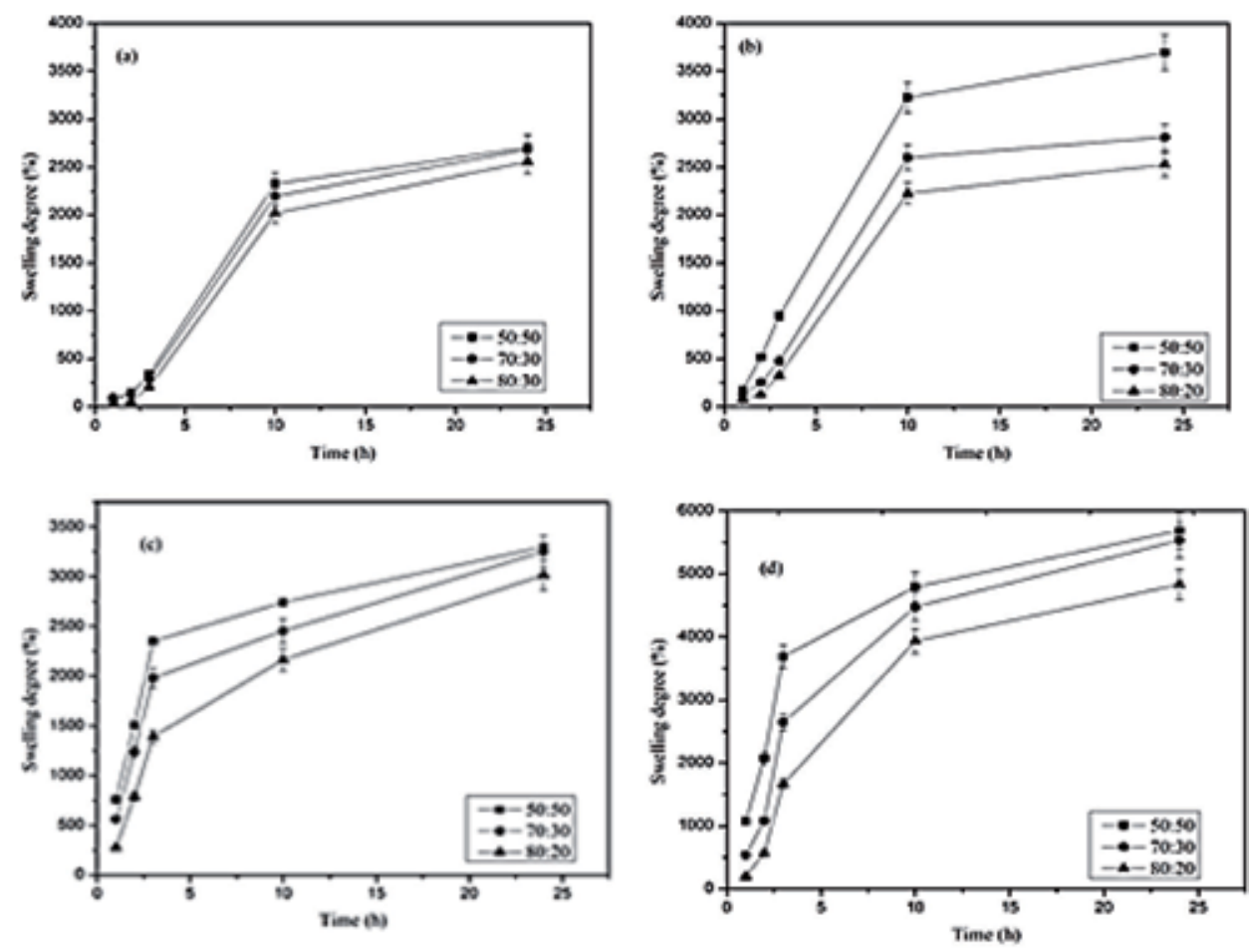

Figure 6.

Effect of alginate/ $\kappa$-carrageenan weight ratio's on the swelling of the alginate/ $\kappa$-carrageenan hydrogel beads with polymer concentrations (a) $1 \%,(b) 1.5 \%,(c) 2 \%$, (d) $2.5 \%$.

alginate/ $\kappa$-carrageenan hydrogel beads has been studied by varying the amount of $\kappa$-carrageenan in the range $20-50 \%(w / w)$. The results showed in Figure 6 shows the swelling degree of alginate/ $\kappa$-carrageenan hydrogel beads increases with increase in carrageenan content. With increase in carrageenan content, the beads become less compact in structure and large pores and surface cavities were observed in the hydrogel beads. The alginate/K-carrageenan (50:50) hydrogel beads show a higher degree of swelling than the alginate/gelatin (50:50) hydrogel beads. Swelling behaviour of hydrogel beads were examined by varying polymer concentration (1, 1.5, 2, 2.5\%). As shown in Figures 5 and 6, increase in polymer concentration leads to enhancement in the swelling degree of both hydrogel beads. In both cases, hydrogel beads with polymer concentration $2.5 \%$ show the highest swelling. Comparison of the swelling degree of alginate/ $\kappa$-carrageenan hydrogel beads with alginate/gelatin hydrogel beads shows that the former one with polymer concentration 2.5\% and weight ratio 50:50 has higher degree of swelling than latter alginate/gelatin hydrogel beads with polymer concentration $2.5 \%$ and weight ratio $50: 50$.

\subsection{Curcumin release study}

\subsubsection{Effect of alginate on curcumin release}

Curcumin release studies were carried out in PBS ( $\mathrm{pH}$ 7.4). Upon variation of sodium alginate ratio in the range $50-80 \%(\mathrm{w} / \mathrm{w})$ in the polymer blend, the amount of curcumin released was found to diminish with time. This observation could be explained on the basis of swelling behaviour of hydrogel beads as discussed earlier. Moreover, by increasing the amount of alginate content, volume fraction of alginate 

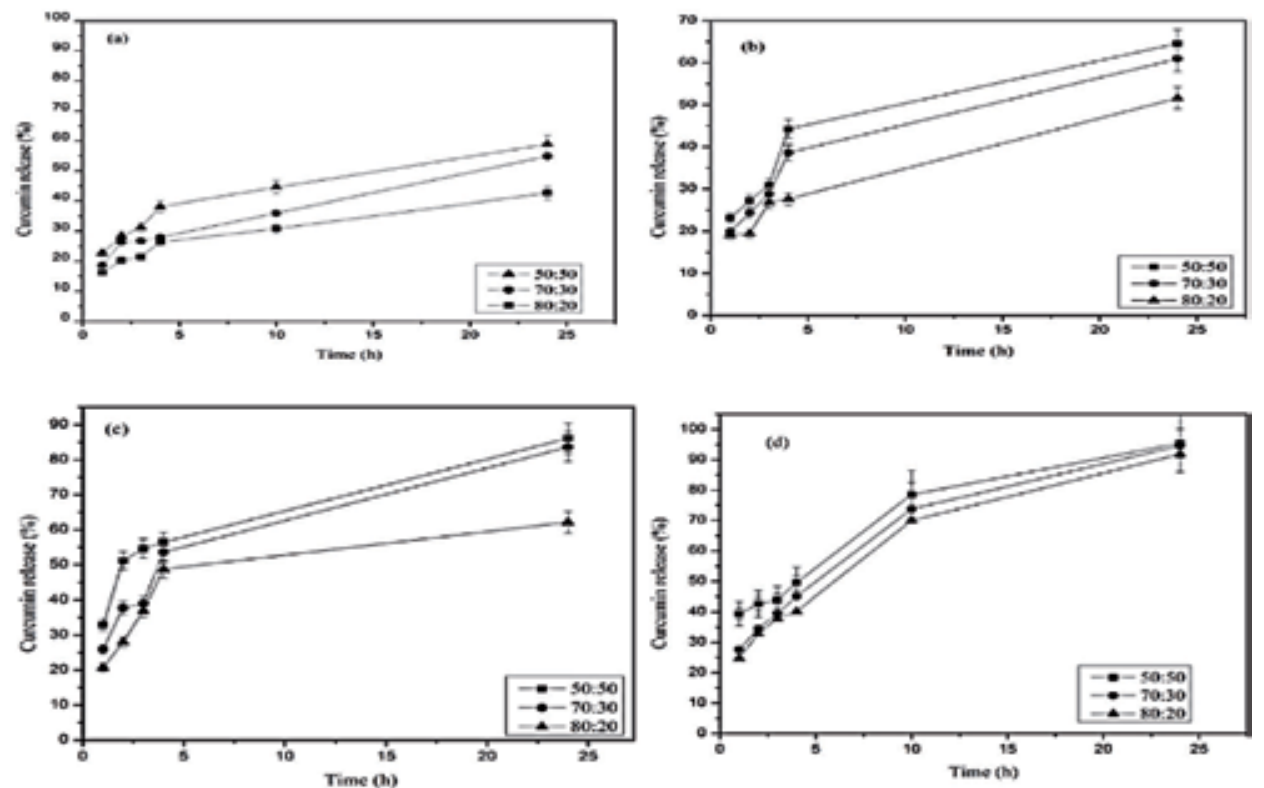

Figure 7.

Effect of alginate/gelatin weight ratio's on cumulative release of curcumin from the hydrogel beads with polymer concentrations (a) $1 \%$, (b) $1.5 \%,(c) 2 \%,(d) 2.5 \%$.

in the hydrogel beads increases leading to the fact that curcumin molecules has to travel a long path in order to diffuse out through the swollen beads because of its characteristic size. This causes a slow release of curcumin from both the hydrogel beads.

\subsubsection{Effect of $\kappa$-Car and gelatin on curcumin release}

$\kappa$-Car content on drug release has been studied by varying $\kappa$-Car in the range $20-50 \%(w / w)$. Figure 4 clearly depicts that the amount of released curcumin increases with increasing $\kappa$-Car content. With increase in $\kappa$-Car content in hydrogel beads, swelling increases resulting in enhanced drug release. Alginate $/ \kappa$-Car beads with alginate/ $\kappa$-Car weight ratio 50:50 showed highest curcumin release percentage (95.45\%), owing to the fact that the matrices were built on weak entanglements driven by the presence of $\kappa$-Car.

\subsubsection{Effect of gelatin on curcumin release}

In the case of alginate/gelatin hydrogel beads, influence of gelatin content on curcumin release was studied by varying its amount in the range $20-50 \%$ (w/w) (Figure 7). Drug release investigations suggests that with increasing gelatin content, release of curcumin from alginate/gelatin hydrogel beads increases and the observed curcumin release is due to the larger swelling. Alginate/gelatin hydrogel beads with 50:50 weight ratios showed $48.24 \%$ curcumin release.

\subsubsection{Effect of varying percentage composition of $\kappa$-Car and gelatin on curcumin release}

Drug release from the hydrogel beads was also studied by varying polymer concentration in the range $1-2.5 \%$. In the case of both hydrogel beads, $2.5 \%$ is the best 
polymer concentration for curcumin release. Curcumin release from alginate/ $/$-Car hydrogel beads shows a higher release percentage than alginate/gelatin hydrogel beads. It can be concluded that higher swelling degree of the alginate/ $\kappa$-Car hydrogel beads leads to greater curcumin release percentage.

\section{Conclusions}

Present study describes the development and evaluation of natural polymer based hydrogel beads for drug encapsulation. The key role of the carrier was for the dissolution of curcumin in aqueous media. Alginate/ $\kappa$-Car hydrogel beads with 50:50 weight ratio showed highest swelling degree having a higher of curcumin release percentage (95.45\%) in PBS ( $\mathrm{pH} 7.4$ ). Observations of morphological analysis show that the amount of carrageenan content has significant content in the pore size of beads. Swelling degree of alginate/gelatin hydrogel beads were lower than compared to the swelling behaviour of alginate/ $\kappa$-Car hydrogel beads. This reveals the role of Car in improving the swelling as well as release pattern of curcumin. Results obtained reiterate the increased efficacy of polymer blends with incorporation of $\kappa$-Car for hydrophobic drug encapsulation. Effective drug release is also established. In fact, the drug release and biological activity of environment friendly hydrogel beads based on natural polymers represent an innovative and adequate alternative for the development of novel therapeutic agents in drug discovery research.

\section{Acknowledgements}

Authors acknowledge financial support from Kerala State Council for Science, Technology and Environment (SAN No. 564/2017/KSCSTE). We thank central instrumentation facility, Sacred Heart College for support and Cochin University of Science and Technology-STIC for instrumental analysis.

\section{Author details}

Reshma Joy, Franklin John and Jinu George*

Biotechnology Laboratory, Department of Chemistry, Sacred Heart College,

Thevara, India

*Address all correspondence to: jinujacob@shcollege.ac.in

IntechOpen

(C) 2019 The Author(s). Licensee IntechOpen. This chapter is distributed under the terms of the Creative Commons Attribution License (http://creativecommons.org/licenses/ by/3.0), which permits unrestricted use, distribution, and reproduction in any medium, provided the original work is properly cited. (cc) BY 


\section{References}

[1] Agarwal S, Mohamed MS, Raveendran S, Rochani AK, Maekawa T, Kumar DS. Formulation, characterization and evaluation of morusin loaded niosomes for potentiation of anticancer therapy. RSC Advances. 2018;8(57):3262132636. Available from: http://xlink.rsc. org/?DOI=C8RA06362A

[2] Lim E-K, Chung B, Chung S. Recent advances in $\mathrm{pH}$-sensitive polymeric nanoparticles for smart drug delivery in cancer therapy. Current Drug Targets. 2016;17(999):1-1. Available from: http://www.eurekaselect. com/openurl/content. php?genre $=$ article $\&$ doi $=10.2174 /$ 1389450117666160602202339

[3] Safari J, Zarnegar Z. Advanced drug delivery systems: Nanotechnology of health design a review. Journal of Saudi Chemical Society. 2014;18(2):85-99. DOI: $10.1016 /$ j.jscs.2012.12.009

[4] John F, George J, Vartak SV, Srivastava M, Hassan PA, Aswal VK, et al.

Enhanced efficacy of pluronic copolymer micelle encapsulated SCR7 against cancer cell proliferation. Macromolecular Bioscience. 2015;15(4):521-534

[5] Blanco E, Shen H, Ferrari M. Principles of nanoparticle design for overcoming biological barriers to drug delivery. Nature Biotechnology. 2015;33(9):941-951

[6] Abedini F, Abraham J. Overview on natural hydrophilic polysaccharide polymers in drug delivery. Polymers Advanced Technologies. Wiley 2017;2018:1-10

[7] John F, George J, Srivastava M, Hassan PA, Aswal VK, Karki S, et al. Pluronic copolymer encapsulated SCR7 as a potential anticancer agent. Faraday Discussions. 2015;177:155-161. DOI: 10.1039/C4FD00176A
[8] Parveen S, Misra R, Sahoo SK. Nanoparticles: A boon to drug delivery, therapeutics, diagnostics and imaging. Nanomedicine: Nanotechnology, Biology and Medicine. 2012;8(2):147-166. DOI: 10.1016/j.nano.2011.05.016

[9] Arroyo E, Luque PA, Cosio M, Soto C, Villarreal R, Nava O, et al. Study of a controlled release polymeric system based on Pluronic P123: Spectroscopic characterization and theoretical model approach. Journal of Molecular Structure. 2017;1138:172176. DOI: 10.1016/j.molstruc.2017.03.018

[10] Senapati S. Controlled drug delivery vehicles for cancer treatment and their performance. Signal Transduction and Targeted Therapy 2017;2018:1-19

[11] Alippilakkotte S, Sreejith L. Pectin mediated synthesis of curcumin loaded poly(lactic acid) nanocapsules for cancer treatment. Journal of Drug Delivery Science and Technology. 2018;48:66-74. DOI: 10.1016/j.jddst.2018.09.001

[12] Zhao D, Zhao X, Zu Y, Li J, Zhang Y, Jiang R, et al. Preparation, characterization, and in vitro targeted delivery of folate-decorated paclitaxelloaded bovine serum albumin nanoparticles. International Journal of Nanomedicine. 2010;5(1):669-677

[13] Rosenblum D, Joshi N, Tao W, Karp JM, Peer D. Progress and challenges towards targeted delivery of cancer therapeutics. Nature Communications. 2018;9(1). DOI: 10.1038/s41467-018-03705-y

[14] Sykes EA, Chen J, Zheng G, Chan WCW. Investigating the impact of nanoparticle size on active and passive tumor targeting efficiency. ACS Nano. 2014;8(6):5696-5706

[15] Yingchoncharoen P, Kalinowski DS, Richardson DR. Lipid-based drug 
delivery systems in cancer therapy: What is available and what is yet to come. 2016;68:701-787

[16] Patra JK, Das G, Fraceto LF, Vangelie E, Campos R, Rodriguez P, et al. Nano based drug delivery systems: Recent developments and future prospects. Journal of Nanobiotechnology. 2018;16: 1-33. DOI: $10.1186 / \mathrm{s} 12951-018-0392-8$

[17] Alshehri A, Grabowska A, Stolnik S. Pathways of cellular internalisation of liposomes delivered siRNA and effects on siRNA engagement with target mRNA and silencing in cancer cells. Scientific Reports. 2018;8(1):1-9

[18] Amjad MW, Kesharwani P, Mohd Amin MCI, Iyer AK. Recent advances in the design, development, and targeting mechanisms of polymeric micelles for delivery of siRNA in cancer therapy. Progress in Polymer Science. 2017;64:154-181. DOI: 10.1016/j. progpolymsci.2016.09.008

[19] Arora R, Kumar R, Agarwal A, Reeta KH, Gupta YK. Comparison of three different extracts of Centella asiatica for anti-amnesic, antioxidant and anticholinergic activities: in vitro and in vivo study. Biomedicine \& Pharmacotherapy. 2018;105:1344-1352. DOI: 10.1016/j.biopha.2018.05.156

[20] Balakrishnan B, Jayakrishnan A. Self-cross-linking biopolymers as injectable in situ forming biodegradable scaffolds. Biomaterials. 2005;26(18):3941-3951

[21] Gopinath V, Saravanan S, Al-maleki AR, Ramesh M, Vadivelu J. Biomedicine and pharmacotherapy: A review of natural polysaccharides for drug delivery applications: Special focus on cellulose, starch and glycogen. Biomedicine \& Pharmacotherapy. 2018;107:96-108. DOI: 10.1016/j.biopha.2018.07.136

[22] Bartelds R, Nematollahi MH, Pols T, Stuart MCA, Pardakhty A, Asadikaram
$\mathrm{G}$, et al. Niosomes, an alternative for liposomal delivery. PLoS One. 2018;13(4):1-18

[23] Moghassemi S, Hadjizadeh A. Nanoniosomes as nanoscale drug delivery systems: An illustrated review. Journal of Controlled Release. 2014;185(1):2236. DOI: 10.1016/j.jconrel.2014.04.015

[24] Bhat PA, Chat OA, Zhang Y, Dar AA. An unprecedented dual responsive gelation of Carbopol induced by Pluronic P123 triblock copolymer. Polymer (Guildf). 2016;102:153-166. DOI: 10.1016/j.polymer.2016.09.013

[25] Bhattarai N, Gunn J, Zhang M. Chitosan-based hydrogels for controlled, localized drug delivery. Advanced Drug Delivery Reviews. 2010;62(1):83-99. DOI: 10.1016/j.addr.2009.07.019

[26] Mészáros M, Porkoláb G, Kiss L, Pilbat AM, Kóta Z, Kupihár Z, et al. Niosomes decorated with dual ligands targeting brain endothelial transporters increase cargo penetration across the blood-brain barrier. European Journal of Pharmaceutical Sciences. 2018;123:228-240. DOI: 10.1016/j. ejps.2018.07.042

[27] Elzoghby AO, Freag MS, Elkhodairy KA. Biopolymeric nanoparticles for targeted drug delivery to brain tumors. In: Kesharwani P, Gupta U editor. Nanotechnology-Based Targeted Drug Delivery Systems for Brain Tumors. Elsevier Inc.; 24 Apr 2018. pp. 169-190. Available from: https://linkinghub.elsevier.com/ retrieve/pii/B9780128122181000075. ISBN: 9780128122181. eBook ISBN: 9780128122495

[28] Gao N, Lü S, Gao C, Wang X, Xu X, Bai X, et al. Injectable shell-crosslinked F127 micelle/hydrogel composites with $\mathrm{pH}$ and redox sensitivity for combined release of anticancer drugs. Chemical Engineering Journal. 2016;287:20-29. DOI: 10.1016/j.cej.2015.11.015 
[29] Gianak O, Pavlidou E, Sarafidis C, Karageorgiou V, Deliyanni E. Silk fibroin nanoparticles for drug delivery: Effect of bovine serum albumin and magnetic nanoparticles addition on drug encapsulation and release. Separations. 2018;5(2):25. Available from: http:// www.mdpi.com/2297-8739/5/2/25

[30] Hyun H, Park J, Willis K, Park JE, Lyle LT, Lee W, et al. Surface modification of polymer nanoparticles with native albumin for enhancing drug delivery to solid tumors. Biomaterials. 2018;180:206-224. DOI: 10.1016/j. biomaterials.2018.07.024

[31] Liang H, He L, Zhou B, Li B, Li J. Folate-functionalized assembly of low density lipoprotein/sodium carboxymethyl cellulose nanoparticles for targeted delivery. Colloids and Surfaces. B, Biointerfaces. 2017;156:1928. DOI: 10.1016/j.colsurfb.2017.05.004

[32] Marianecci C, Di Marzio L, Rinaldi F, Celia C, Paolino D, Alhaique F, et al. Niosomes from 80s to present: The state of the art. Advances in Colloid and Interface Science. 2014;205:187-206. DOI: $10.1016 /$ j.cis.2013.11.018

[33] Qin Y, Tian Y, Liu Y, Li D, Zhang $\mathrm{H}$, Yang $\mathrm{Y}$, et al. Hyaluronic acidmodified cationic niosomes for ocular gene delivery: Improving transfection efficiency in retinal pigment epithelium. The Journal of Pharmacy and Pharmacology. 2018;70(9):1139-1151

[34] Rajera R, Nagpal K, Singh SK, Mishra DN. Niosomes: A controlled and novel drug delivery system. Biological \& Pharmaceutical Bulletin. 2011;34(7):945-953. Available from: https://www.jstage.jst.go.jp/article/ bpb/34/7/34_7_945/_article

[35] Gu D, O’Connor AJ, Qiao GGH, Ladewig K. Hydrogels with smart systems for delivery of hydrophobic drugs. Expert Opinion on Drug Delivery. 2017;14(7):879-895. DOI: 10.1080/17425247.2017.1245290
[36] Blount RP, Bhattarai N. Natural Polysaccharide-Based Hydrogels for Controlled Localized Drug Delivery. Gels Handbook. 2016;35-59. DOI:10.1142/9789813140417_0002

[37] Chen J, Huang GD, Tan SR, Guo J, $\mathrm{Su} \mathrm{ZQ}$. The preparation of capsaicinchitosan microspheres (CCMS) enteric coated tablets. International Journal of Molecular Sciences. 2013;14(12):24305-24319

[38] Liu Z, Jiao Y, Wang Y, Zhou C, Zhang Z. Polysaccharides-based nanoparticles as drug delivery systems. Advanced Drug Delivery Reviews. 2008;60(15):16501662. DOI: 10.1016/j.addr.2008.09.001

[39] Sathuvan M, Thangam R, Gajendiran M, Vivek R, Balasubramanian S, Nagaraj $\mathrm{S}$, et al. $\kappa$-Carrageenan: An effective drug carrier to deliver Curcumin in cancer cells and to induce apoptosis. Carbohydrate Polymers. 2016;160:184193 DOI: 10.1016/j.carbpol.2016.12.049

[40] Malafaya PB, Silva GA, Reis RL. Natural-origin polymers as carriers and scaffolds for biomolecules and cell delivery in tissue engineering applications. Advanced Drug Delivery Reviews.Elsevier 2007;59:207-233

[41] Yadav G, Sharma N, Bansal M. Thakur N, Application of natural polysaccharide for delivery of biopharmaceuticals. International Journal of pharmacy \& Life Sciences. 2013;4(6):2756-2765

[42] Boateng J, Burgos-Amador R, Okeke O, Pawar H. Composite alginate and gelatin based bio-polymeric wafers containing silver sulfadiazine for wound healing. International Journal of Biological Macromolecules. 2015;79:63-71. DOI: 10.1016/j. ijbiomac.2015.04.048

[43] Shimizu T, Yamato M, Kikuchi A, Okano T. Cell sheet engineering for myocardial tissue reconstruction. In: Leong K editor. The Biomaterials: Silver 
Preparation and Characterisation of Niosomal Emulsions as Novel Drug Delivery Vehicle...

DOI: http://dx.doi.org/10.5772/intechopen.86942

Jubilee Compendium. Vol. 24. Elsevier. 2006. pp. 211-218. ISSN: 0142-9612

[44] Pankongadisak P, Ruktanonchai UR, Supaphol P, Suwantong O.

Development of silver nanoparticlesloaded calcium alginate beads embedded in gelatin scaffolds for use as wound dressings. Polymer International. 2015;64(2):275-283

[45] Cuadros TR, Erices AA, Aguilera JM. Porous matrix of calcium alginate/ gelatin with enhanced properties as scaffold for cell culture. Journal of the Mechanical Behavior of Biomedical Materials. 2015;46:331-342

[46] John F, George JUK. Curcumin encapsulated alginate/Pluronic block copolymer micelles as a promising therapeutic agent. UK Journal of Pharmaceutical and Biosciences. 2014;2(3):6-12. Available from: www. ukjpb.com

[47] Mei L, He F, Zhou RQ, De Wu C, Liang R, Xie R, et al. Novel intestinal-targeted Ca-alginate-based carrier for $\mathrm{pH}$-responsive protection and release of lactic acid bacteria. ACS Applied Materials \& Interfaces. 2014;6(8):5962-5970

[48] Santoro M, Tatara AM, Mikos AG. Gelatin carriers for drug and cell delivery in tissue engineering. Journal of Controlled Release. 2014;190:210-218. DOI: 10.1016/j.jconrel.2014.04.014

[49] Ganta S, Devalapally H, Shahiwala A, Amiji M. A review of stimuliresponsive nanocarriers for drug and gene delivery. Journal of Controlled Release. 2008;126(3):187-204

[50] Lázaro N, López Sevilla A, Morales S, Marqués AM. Heavy metal biosorption by gellan gum gel beads. Water Research. 2003;37(9):2118-2126 



\title{
Solid Lipid Based Nano-particulate Formulations in Drug Targeting
}

\author{
Bibhash Chandra Mohanta, Subas Chandra Dinda, \\ Narahari Narayan Palei and Jyotirmoy Deb
}

\begin{abstract}
Recently, targeted drug delivery systems have gained much more interest for delivering varieties of drugs as well as imaging agents specifically to the targeted disease cells or tissues. These are well known for their increased precision and accuracy in mode of drug delivery along with reduced side effects. Though numerous carriers are being employed for drug targeting, the solid lipid based nanoparticles (SLNs) are preferred over them owing to their ability to encapsulate wide varieties of drugs, biocompatibility, ease of surface modification, scaling up feasibility, and possibilities of both active as well as passive targeting to various organs. Surface of these drug loaded SLNs can be modified by conjugating different ligands to enhance their tissue/organ targeting ability and therapeutic efficacy to much higher extent. In this chapter, we have discussed about the SLNs and their different surface modified forms for passive as well as active targeting to different organ such as (colon, breast, lungs, liver, kidney, brain, eyes, etc.) in combating different diseases.
\end{abstract}

Keywords: SLNs, targeted drug delivery, imaging, colon, breast, eye, lungs, liver, kidney, brain

\section{Introduction}

Drug delivery refers to the approaches or methods or technologies of administering or transporting active pharmaceutical ingredient(s) and other xenobiotics through different routes for achieving the desired therapeutic effect in human or animal safely. The pharmacokinetics and pharmacodynamics issues of drugs are the most important considerations of drug delivery which is profoundly integrated with dosage form and route of administration. The kinetics of drug release, drug concentration profile in the plasma, onset of action, duration of action, site of action, and side effects of a drug deeply influenced by the DDS.

\section{Conventional vs. targeted drug delivery systems}

\subsection{Conventional drug delivery systems}

Conventional drug delivery system is also known as classical drug delivery system or traditional drug delivery system, which sometimes unable to maintain the 
steady-state plasma concentration as desired for a specific time period and may not be able to deliver the drugs to the specific site of organ or tissues may be because of barriers in transportation for which it may be needed to administer with multiple doses at a regular time interval or need to go for targeted drug delivery systems.

\subsection{Targeted drug delivery systems}

Targeted drug delivery system (TDDS) is popularly known as smart drug delivery system. The aim of the TDDS is to localize, target and to have a protected interaction of a drug with the diseased cells/tissues of interest for a prolonged period of time. TDDS helps in maintaining the requisite plasma and tissue drug levels in the body and protects the healthy tissues from damage may be some times caused by the drugs [1]. It offers various benefits over conventional DDS such as localization of a drug to the desired or specific site, enhancement of therapeutic efficacy, reduction in the dosing frequency and toxic side effects, controlled biodistribution of drug, modulated pharmacokinetics, and improved patient compliances [2]. The TDDS is a highly integrated DDS which needs the coordinated effort from various experts such as biologist, chemist, engineers for its fabrication and optimization.

\subsection{Nanotechnology vs. drug delivery systems}

Nanotechnology is defined as the technology which allows studying, controlling, manipulating and manufacturing of structures or devices in the nanoscale. It is a multi-disciplinary scientific field applying engineering and manufacturing principles at the molecular level. These nanosized objects/structures/devices, e.g. "nanoparticles" exhibit unique properties and function that distinctly differ from those seen from the items made up from the same materials. Nanomaterials possess many unique characteristics such as mechanical, optical, magnetic, electrical, and biochemical, which provoke them to intermingle with complex cellular functions in an exceptional manner [3].

Since its introduction in 1959, the nanotechnology brought a great revolution in all areas of sciences and particularly in drug formulation and drug delivery system design. Nanomedicine is the medical application of nanotechnology, which plays an imperative role in the medical biology, diagnosis, monitoring, prevention and treatment of diseases. Since last few decades, owing to the rapid developments in nanotechnology and carrier materials, a great advancement in the nanoparticulate DDS has been noticed and they are taking the lead among all types of DDS [4].

The nanoparticulate DDS possesses numerous advantages such as higher intracellular uptake (cells and tissues have a greater affinity and acceptability to the nanoparticles as compared to micro/macro molecules), ability to penetrate into submucosal layer (nanometric size), greater suitability for administration through the systemic circulation (nanometric size), greater feasibility/flexibility to develop into a targeted DDS for targeting various sites/organs. Thus, the nanometric size, tailored surface, and cross functionality of these nanoparticles will continue to explore many unexplored research areas and may help in designing and developing new biomedical applications [5].

\subsection{Colloidal drug carrier and solid lipid nanoparticles}

The term "colloid" is applied to the dispersed system where the dispersed phase particles size are very fine and generally below $1 \mu \mathrm{m}$. Thus, the biphasic drug carrier containing very fine dispersed phase particles $(<1 \mu \mathrm{m})$ which sequester, transport 
and retain the active drug en route, while they deliver the drug within or in the vicinity of a target is popularly known as colloidal drug carrier. These colloidal drug carriers comprise nanoparticles, liposome, niosome, nanospheres, multiple emulsion, and nanosuspensions, etc. [6]. Colloidal carriers aid in solubilization of lipophilic drug, protect the sensitive drug from degradation in biological fluid, reduce toxic side effect, improve patient compliances, prolong the duration of action and drug targeting potentiality [7].

Though the polymeric nanoparticulate DDS have shown hugely impressive performance for providing therapeutic benefits in the case of long term delivery of a therapeutic agent, but still, the number of polymeric nanoparticulate formulations in the market is still limited. This is because of polymeric toxicity, high cost of polymers, and lack of feasibility for scaling up. Lipid based nanoparticulate DDSs are proposed as an alternative to polymeric nanoparticulate DDS and gained tremendous attention in the field of nanomedicine. These comprise liposomes, niosomes, nanoemulsions, solid lipid nanoparticles (SLNs) and nanostructured lipid carrier (NLCs), etc. [5].

SLNs are the second generation lipid nanocarriers that overcome most of the limitations associated with conventional drug delivery system and other colloidal lipid/polymeric nano carriers. It promises to offer numerous benefits including biocompatibility and biodegradability, physiochemical stability, lower toxicity, ability to incorporate both hydrophilic and lipophilic drugs, improved bioavailability, enhanced in vitro and in vivo stability of drugs, controlled-release characteristics, site specificity in drug delivery as well as feasibility in pilot scale up along with its suitability in drug delivery through different routes of administration [4].

\subsection{Solid lipid nanoparticles and drug targeting}

In the emerging field of nanomedicine, SLNs is at the forefront. It is made up from biocompatible/physiological lipids (e.g. partial glycerides, triglycerides, fatty acids, wax, and steroids) that remain in solid form at room temperature. Numerous techniques are being developed for the fabrication of SLNs using the biocompatible/ physiological lipid which has records of innocuous use in medicine [8]. Apart from drugs, the essential materials for fabricating SLNs are solid lipids as matrix materials, emulsifiers, stabilizers, and water. The nanometric size and larger surface area of SLNs is suitable to be embedded with some potential functionalized ligands, antibodies, moieties, and other functional groups that help in drug targeting [5].

The real success of lipid nanoparticles relies on the development of dosage forms that are able to improve the therapeutic index of the drugs by mounting their concentration specifically at the targeted site or organs. Drugs can be incorporated in SLNs which lead to offer a new model in drug delivery that could be applied for drug targeting. The therapeutic payload of various categories of drugs (such as antiinfective, anticancer drugs, anti-inflammatory, etc.), antigens, proteins, and nucleotides can be enhanced in specific site and organs by associating with SLNs. On another side, SLNs face numerous challenges which include rapid clearance, serum instability (dependent on the specific formulation) and nonspecific uptake by the mononuclear phagocytic system (play a major role for opsonizing the foreign particles and remove SLNs from the circulation) [9]. The above mentioned limitations can be nullified by conjugating different ligands to the surface of SLNs which could help to increase the circulation time and targeted delivery of the drug to the specific site. The targeting properties to a specific site can be further enhanced by selecting surface markers [10]. Thus, in this article, we focused on SLNs and various ligand conjugated SLNs which act as suitable carriers for targeting to different sites such as lungs, brain, liver, breast, eyes, colon, kidney, etc. 


\section{Solid lipid nanoparticles for lungs targeting}

Targeted delivery of a drug to the lungs is gaining much more interest at the present time, for the treatment of lungs cancer, tuberculosis, and other airborne diseases where lungs are the primary site of action or site administration of drugs [11]. In order to get maximum therapeutic benefits from lungs delivery, a suitable DDS with appropriate physicochemical properties are necessary and SLN along with ligand conjugated SLN are the most fitted on this ground.

\subsection{Solid lipid nanoparticles for active lungs targeting}

The lungs offer a very high surface area for rapid absorption of drugs owing to high vascularization and avoidance of the first pass effect. Sometimes, targeted delivery of certain drugs to the lungs is very important not only for improving the bioavailability and therapeutic activity but also for reducing the systemic side effects [5].

To achieve a prolonged hypoglycemic effect, Liu et al. developed insulin-loaded nebulized SLNs which were administered through intrapulmonary route. The hypoglycemic effects, stability of SLN during nebulization, and deposition pattern of the drug were evaluated. SLNs exhibited excellent protective effect for insulin against degradation or leakage from nanospheres and were relatively stable during nebulization via jet nebulizer. Nebulized insulin SLNs helped in improving the bioavailability and showed significantly higher hypoglycemic effect as compared to insulin phosphate buffer solution administered through pulmonary route. Thus nebulized insulin-SLNs could be a promising DDS for the treatment of diabetes [12]. In another study $\mathrm{Bi}$ et al. successfully developed freeze dried insulin-SLNs suitable for intra-tracheal administration through a spray. High preservation of insulin was noticed in SLNs after spray freeze drying. They had performed in vivo studies on diabetic rat and observed a prolonged hypoglycemic effect [13].

Rifampicin (RIF) were successfully encapsulated into the SLNs that delivered RIF specifically to the alveolar macrophage (AM) with strong antimycobacterial efficacy (MIC reduced to 1/8 fold than that of the free drug). Generally, mycobacterium safely multiplies in the AM (acts as an incubator), as the mycobacterium is resistant to the biocidal mechanism of AM. The developed SLNs were more stable and the particle sizes were very much suitable for improving RIF's uptake by AMs which are particle size dependent [14]. Similarly, Rifabutin loaded SLNs significantly improved uptake of the drug by the macrophages which were demonstrated in an in vitro model [15].

Co-administration of RIF and isoniazid through SLN formulation significantly reduced (60\%) degradation of RIF (from 48.81 to $12.35 \%$ ) from acidic gastric $\mathrm{pH}$ owing to the presence of isoniazid. The developed SLNs promoted targeted delivery of drug to the brain with enhanced bioavailability and lesser side effect that could be helpful in the case of cerebral tuberculosis [16].

Significantly higher biodistribution of dexamethasone acetate (DXM) to the lungs was achieved through intravenous administration of DXM loaded SLNs. The area under curve (AUC) of DXM- SLNs was increased by 17.8-fold as compared to DXM-solution. The maximum concentration of the DXM in the lungs was observed at $0.5 \mathrm{~h}$ post DXM-SLNs injection [17].

Similarly higher biodistribution of amikacin (AMK) in the lungs was achieved by pulmonary administration of AMK-SLNs as compared to the free drug administered through i.v., which could be helpful in the treatment of cystic fibrosis [18]. 


\subsection{Ligand conjugated solid lipid nanoparticles for passive lungs targeting}

Lungs targeted delivery of drugs is a challenging task due to the mucociliary clearance. In this regard, the ligand-anchored DDS not only proves its potential in achieving improved site-specific drug delivery, but also it reduces the chances of drug uptake by reticulo-endothelial system (RES). It is believed to play a major role in congenital defense and exhibit diversified biological activities such as antimicrobial, anticancer, immunomodulation, an exertion to control cell growth, binding, and inhibition of numerous biologically active compounds. However, clinical success of such approaches relies on the choice of appropriate ligand free from immunogenic potential with the potential to provoke cargo internalization by the target cell [19].

The mechanism of receptor mediated endocytosis of ligand anchored SLNs and drug release technique has been shown in Figure 1.

\subsubsection{Lactoferrin (Lf) conjugated solid lipid nanoparticles}

In lung associated diseases, receptors of Lactoferrin (80-kDa iron-binding glycoprotein) is overexpressed in the lungs. Thus, Lf conjugated DDS may become a promising tool for targeted delivery of drugs to lungs in lung-associated diseases [20]. Rifampicin (RIF) loaded SLNs were successfully prepared and were coupled with Lf via carbodiimide chemistry i.e., coupling of the Lf carboxylic group with the stearylamine amine group present on the surface of the previously formed RIF loaded SLNs in the presence of N-ethyl-N-(dimethylaminopropyl)-carbodiimide (EDC). An in vivo biodistribution study revealed 3.05 time higher drug uptakes by the lungs in case of Lf-RIF-SLNs as compared to unconjugated RIF-SLNs. It was further confirmed from fluorescence photomicrographs that clearly showed access of the Lf-coupled SLNs into the lung. Thus, lactoferrin is an efficient molecule that can be used for targeting active agents directly to the lungs [21].

\subsubsection{Wheat germ agglutinin (WGA) conjugated solid lipid nanoparticles}

Conjugation of bioadhesive ligand molecules with SLNs helps in improving drug absorption/bioavailability by increasing residence time in the GIT and reducing

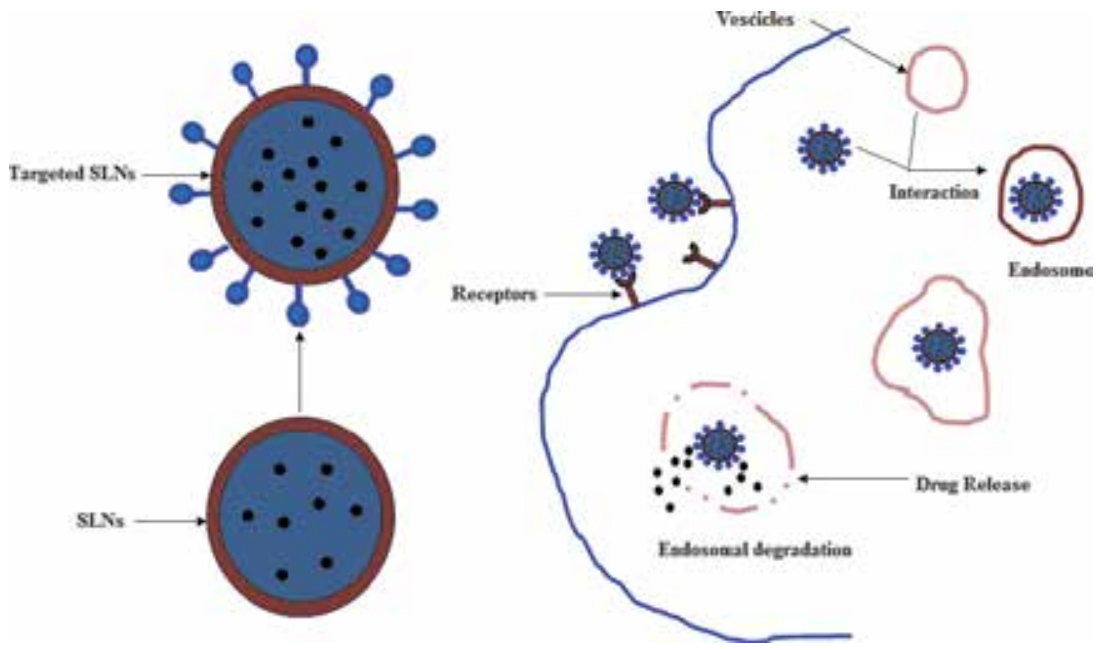

Figure 1.

Mechanism of receptor mediated endocytosis of ligand anchored SLNs and drug release technique. 
dosing frequency. Lectins (a group of diverse proteins/glycoproteins) are the bioadhesive ligand and have stable structure and receptor binding ability. It offers resistance to enzymatic digestion/degradation, which helps in its in vivo survival for a prolonged time period [22]. Wheat germ agglutinin (WGA) is an example of nontoxic plant lectin which binds particularly to $\mathrm{N}$-acetyl-D-glucosamine and sialic acid on cell surfaces. Conjugation of WGA with SLNs could be helpful for targeted delivery of antitubercular drugs to the lungs.

WGA conjugated SLNs loaded with rifampicin (WRSLNs) were successfully developed for lungs specific delivery of rifampicin (RIF). The conjugation of WGA to RIF loaded SLN was carried out by two-steps carbodiimide reaction reported by Ertl et al. with slight modification [23]. Even after conjugation with the SLNs, the WGA retained its bio-recognition activity and sugar-binding specificity [24]. From the in vivo study result, the interaction of WRSLNs with porcine mucin was confirmed. However, non-conjugated SLNs did not show any conjugation with the porcine mucin. The WRSLNs were stable at refrigerated temperature and also in presence of electrolytes up to $1.0 \mathrm{M} \mathrm{Na}_{2} \mathrm{SO}_{4}$ concentration. The WRSLNs were 10 times more stable than RIF-SLN which could be owing to the steric stabilization by lectin present on nanoparticle surface. Prepared WRSLNs had shown narrow size distribution, controlled release of a drug, retention of biorecognition activity and improved physical stability of drug against electrolyte induced flocculation [25].

\subsubsection{Mannose conjugated solid lipid nanoparticles}

Studies on tuberculosis revealed overexpression of the mannose receptors specifically on alveolar macrophages (AM) surfaces [26]. Keeping it in mind attempts were taken to develop mannosylated SLN to deliver antitubercular drugs targeting to alveolar macrophages which have higher affinity for mannose. Rifabutin loaded mannosylated SLNs were successfully developed. Manosylation was done by ring opening reactions followed by reaction of aldehyde groups of mannose in $0.1 \mathrm{M}$ sodium acetate buffer ( $\mathrm{pH} 4.0)$ with the amino groups of lipid. This leads to formation of Schiff's base ( $-\mathrm{N}=\mathrm{CH}-)$, which may then get reduced to secondary amine $\left(-\mathrm{NH}-\mathrm{CH}_{2}-\right)$ and remain in equilibrium with Schiff's base at basic $\mathrm{pH}$. In vivo studies were conducted to evaluate for their cytotoxicity, targeting potential, AM uptake, and hematological studies. It was reported that mannosylation improved uptake (up to 6-fold) of rifabutin by the AM. Moreover, mannosylated rifabutinSLNs were less immunogenic and helped in sustained delivery of drug. Thus, the mannosylated SLNs may be employed for AM targeted delivery of rifabutin for effective management of TB [27].

Overexpression of mannose receptors in case of lungs cancer was reported by numerous investigators. The mannosylated-distearoyl phosphatidyl-ethanolamine SLNs loaded with paclitaxel (PTX) was developed for lungs targeted delivery of PTX. Manosylation was done by ring opening reaction followed by reaction of an aldehyde group of mannose with the free amine group provided by stearylamine and DSPE in sodium acetate buffer $(\mathrm{pH} 4.0)$. The stability testing data indicated that SLNs formulations stored at $4 \pm 2{ }^{\circ} \mathrm{C}$ were more stable than those stored at $27 \pm 2^{\circ} \mathrm{C}$. It was revealed that mannosylated SLNs deliver significantly higher concentration of drug to the alveolar cell sites and showed improved antiproliferative efficacy as compared to PTX solution and PTX-SLNs [28].

\subsubsection{Folate conjugated solid lipid nanoparticles}

The folate receptors ( $\alpha$-form) are overexpressed on the surface of lung tumor cells. The extents of the overexpression are different in different types of lung tumors (adenocarcinomas-72\%, squamous cell carcinomas- $51 \%$, small cell 
Solid Lipid Based Nano-particulate Formulations in Drug Targeting

DOI: $h$ ttp://dx.doi.org/10.5772/intechopen. 88268

\begin{tabular}{|c|c|c|c|c|c|c|c|c|}
\hline $\begin{array}{l}\text { Sl. } \\
\text { no }\end{array}$ & $\begin{array}{l}\text { SLN } \\
\text { (Type) }\end{array}$ & Lipids & $\begin{array}{l}\text { Preparation } \\
\text { method }\end{array}$ & Drugs & Target & Model & Comments & Ref. \\
\hline 01 & SLN & SA, PA & $\begin{array}{l}\text { Reverse micelle- } \\
\text { double emulsion }\end{array}$ & Insulin & Lungs & $\begin{array}{l}\text { Male SD } \\
\text { rats }\end{array}$ & $\begin{array}{l}\text { Improved hypoglycemic } \\
\text { effect }\end{array}$ & {$[12]$} \\
\hline 02 & SLN & SA, PA & $\begin{array}{l}\text { Modified lipid } \\
\text { film hydration } \\
\text { method }\end{array}$ & RIF & $\mathrm{AM}$ & $\mathrm{SD}$ rats & $\begin{array}{l}\text { Improved drug uptake } \\
\text { by AMs. }\end{array}$ & {$[14]$} \\
\hline 03 & SLN & $\begin{array}{l}\text { SL, } \\
\text { GTS }\end{array}$ & $\begin{array}{l}\text { Ultrasonication } \\
\text { with high } \\
\text { pressure } \\
\text { homogenization }\end{array}$ & DXM & Lungs & Mice & $\begin{array}{l}\text { Improved therapeutic } \\
\text { efficacy targeting to } \\
\text { lungs }\end{array}$ & [17] \\
\hline 04 & $\begin{array}{l}\text { Lf- } \\
\text { SLNs }\end{array}$ & SL & $\begin{array}{l}\text { Solvent injection } \\
\text { method }\end{array}$ & RIF & LfR & $\begin{array}{l}\text { Albino } \\
\text { rats }\end{array}$ & $\begin{array}{l}\text { Targeted delivery of RIF } \\
\text { to Lungs }\end{array}$ & {$[21]$} \\
\hline 05 & $\begin{array}{l}\text { WGA- } \\
\text { SLN }\end{array}$ & $\begin{array}{l}\text { SA, } \\
\text { GMS }\end{array}$ & $\begin{array}{l}\text { Single } \\
\text { emulsification } \\
\text { followed by } \\
\text { solvent } \\
\text { evaporation }\end{array}$ & RIF & Mucin & Porcine & Controlled release of RIF & {$[25]$} \\
\hline 06 & $\begin{array}{l}\text { Msy- } \\
\text { SLN }\end{array}$ & TSN & $\begin{array}{l}\text { Modified solvent } \\
\text { injection method }\end{array}$ & RFB & MR & $\begin{array}{l}\mathrm{J} 774 \text { \& } \\
\text { albino } \\
\text { rats }\end{array}$ & $\begin{array}{l}\text { AM specific drug } \\
\text { delivery. Enhanced } \\
\text { therapeutic efficacy. }\end{array}$ & {$[27]$} \\
\hline 07 & $\begin{array}{l}\text { Msy- } \\
\text { SLN }\end{array}$ & $\begin{array}{l}\text { TSN, } \\
\text { DSPE }\end{array}$ & $\begin{array}{l}\text { Modified solvent } \\
\text { injection method }\end{array}$ & PTX & MR & $\begin{array}{l}\text { A549 \& } \\
\text { Male } \\
\text { albino } \\
\text { rat }\end{array}$ & $\begin{array}{l}\text { Alveolar cell site } \\
\text { delivery of drug with } \\
\text { enhanced anti- } \\
\text { proliferative efficacy. }\end{array}$ & {$[28]$} \\
\hline 08 & Ft-SLN & $\begin{array}{l}\text { CHO, } \\
\text { GS }\end{array}$ & $\begin{array}{l}\text { Nano } \\
\text { precipitation } \\
\text { method }\end{array}$ & PTX & FR & $\begin{array}{l}\text { HeLa \& } \\
\text { M109- } \\
\text { HiFR } \\
\text { cells }\end{array}$ & $\begin{array}{l}\text { Prolonged drug } \\
\text { residence time, reduced } \\
\text { MIC }\end{array}$ & {$[30]$} \\
\hline
\end{tabular}

Table 1.

List of SLNs and their different ligand conjugated forms for lungs cells targeting.

carcinoma-25\%, and lung metastases-30\%) [29]. These receptors allow folate derivatives to bind preferentially that permits intracellular incorporation of folate derivative by endocytosis.

Folate-conjugated copolymer of polyethylene glycol (PEG) and N-[(2-hydroxy3-trimethylammonium) propyl] chitosan chloride (HTCC) SLNs loaded with Paclitaxel (PTX) were successfully developed. The conjugation of folate- conjugated PEG and HTCC to RIF-SLN was carried out by carbodiimide mediated coupling chemistry. Pulmonary administration of the developed F-PEG-HTCC-SLNs selectively delivered the PTX to the lung's cancer cells with improved penetrability and prolonged lung residence. Moreover, the developed SLN significantly reduced the in vitro half-maximum inhibitory concentration of PTX in M109-HiFR cells [30].

List of SLNs and their different ligand conjugated forms for lungs targeting have been summarized in Table 1.

\section{Solid lipid nanoparticles for brain targeting}

Targeted delivery of drugs to the brain for is gaining much more interest at the present time, not only for the treatment of brain tumor and other neurodegenerative disorders but also for their diagnosis. Brain targeted delivery of drugs is the most challenging task because of the presence of strongest physiological barrier, i.e., 
blood brain barrier (BBB). It is a highly selective semipermeable membrane barrier constituted by specialized microvascular endothelial cells, basement membrane and glial cells (astrocytes, neurons, and pericytes). As long as the BBB remains integral, the drugs remain ineffective in the brain. Though $\mathrm{BBB}$ is a major issue for it, yet it offers scores of opportunities such as presence of numerous transport proteins and specialized receptors [5].

Conventional approach for the treatment of brain tumor and other brain-related disorders needs a higher dose of the drug that leads to systemic toxicity and substantial adverse effects on CNS and vital normal tissues. However, various researchers have reported SLNs to be a suitable DDS targeting the brain as the SLNs possess numerous unique characteristics, such as improved uptake of SLN by the brain due to lipidic nature, bioacceptability and biodegradability nature, non-toxic, nano sized particles suitable for prolonged circulation time in blood scale up feasibility, absence of burst drug release effect [31]. Thus, SLN could be used as potential as well as promising candidate for brain targeting.

\subsection{Solid lipid nanoparticles for active brain targeting}

SLNs are the most acceptable brain targeted DDS employed in brain tumor owing to their ability to escape and/or inhibit P-glycoprotein in the blood-brain barrier [32]. Camptothecin (CMP) loaded SLN were successfully developed for the treatment of glioma. Encapsulation of drug in phospholipids and conjugation with the peptide enhances the permeation of drug across BBB, which leads to brain targeted delivery of the drug. The developed SLN was stable in terms of size and charge within a year of storage which might be due to appropriated acidic $\mathrm{pH}$ (inclusion of behenic acid into the lipid core of the SLNs). DMPC (1,2-dimyristoyl-sn-glycero-3-phosphocholine) membrane helps in efficient release of the incorporated CMP into the brain parenchyma crossing the BBB. Against glioma, the developed SLNs showed higher cell death or antitumor activity using the lowest maximal inhibitory concentration (IC50) values. Biodistribution study revealed higher accumulation of CMP in the brain in the case of SLNs as compared to other non-encapsulated drugs. However, pharmacokinetics study revealed lower deposition of CMP in peripheral organs indicating lesser toxicological effects in the vital organ. Thus, in short, the SLNs exhibited enhanced accumulation, distribution, and retention of camptothecin in the animal brain along with superior in vitro antitumor activity against glioma [33].

Riluzole (RLZ), a potent neuroprotective agent is useful in the treatment of neurodegeneration including traumatic brain injury and amyotrophic lateral sclerosis. RLZ-SLNs were able to deliver the riluzole successfully to the brain which helps in preventing acute cell damage induced by glutamate in neurodegenerative diseases of motor neurons. Moreover, it also protects dopamine neuron in the case of Parkinson's disease. Stability studies on RLZ-SLNs were also carried out at $\mathrm{pH} 1.1$, 5.5 and 7.4, and in human plasma which revealed the absence of riluzole degradation in all the investigated media [34].

Vinpocetine (VIN), a derivative of vincamine alkaloid, useful against chronic cerebral vascular ischemia. VIN loaded SLN were successfully developed and achieved the objectives of delivering the drugs to the brain. Release kinetics of the developed SLN followed zero-order sustained release profile [35].

\subsection{Ligand conjugated solid lipid nanoparticles for passive brain targeting}

\subsubsection{Lactoferrin conjugated solid lipid nanoparticles}

Augmentation of drug uptake into the brain is a subtle mission in the treatment of brain tumor. Lactoferrin (Lf) receptor present on the BBB in different species 
along with the cell surface of glioblastomas [36]. Keeping it in mind, various researchers tried to target lactoferrin receptor through conjugation of lactoferrin with SLNs for active targeting to brain tumor. Moreover, this conjugated system showed higher stability, and drug payload.

Lactoferrin (Lf) conjugated SLNs loaded with docetaxel (DTX) were able to show effective brain targeting efficiency. Carbodiimide chemistry was employed for conjugation of Lf on SLN surface (Lf-SLN). Receptor saturation studies and distribution studies of lipidic nanoparticles in the brain indicated brain targeting mechanism for uptake in brain tumor cell and brain respectively. The Lf-SLNs were more stable. It not only showed significantly higher DTX concentration in the brain but also showed superior apoptotic activity when compared with unconjugated SLNs and DTX. Thus it was confirmed that conjugation of Lf to SLN significantly improved the targeting potential of the DTX for brain tumor [37].

\subsubsection{Tamoxifen-Lactoferrin cross conjugated solid lipid nanoparticles}

P-glycoprotein (P-gp) and multidrug resistance-associated proteins (MRAPs) are localized on brain microvascular endothelial cells (BMEC) which pump out the substances/drug out from the central nervous system. Tamoxifen (Tf), a selective estrogen receptor modulator possibly reverse the capability of efflux transporters like MRAPs in cancer cells [38]. Thus, incorporation of Tf could help in preventing a wide range of medication from efflux loss. Lactoferrin (Lf) receptors are overexpressed in BMEC and glioblastoma multiforme (GBM) cells. It is reported that the Lf cause inhibition to the multiplication of malignant GBM cells.

Tamoxifen (Tx) and lactoferrin (Lf) cross-conjugated carmustine (CRM)loaded SLNs were developed. For conjugation of Tx on CRM-SLN, the carbonyl groups of the SLN were first activated with $0.1 \%(\mathrm{w} / \mathrm{v})$ carbodiimide and $0.05 \%$ $(\mathrm{w} / \mathrm{v}) \mathrm{N}$-hydroxysuccinimide. The suspended SLNs were then crosslinked with $0.05 \%, 0.1 \%, 0.15 \%$, or $0.2 \%(\mathrm{w} / \mathrm{v}) \mathrm{Tx}$ at $150 \mathrm{rpm}$ and $25^{\circ} \mathrm{C}$ for $3 \mathrm{~h}$ and centrifuged. Further Lf conjugation was done by reacting Lf $(0.02 \%, 0.04 \%, 0.06 \%$, or $0.08 \%)$ $(\mathrm{w} / \mathrm{v})$ with the activated CRM-SLNs and Tx-CRM-SLN. The conjugated SLNs were more stable which could be due to inclusion of behenic acid into the lipid core. These were efficiently penetrated through a monolayer of human BMEC and human astrocytes and to target GBM cells. A 10-fold increase in the permeability of $\mathrm{BBB}$ and improved the sustained release of CRM was achieved with the help of the developed SLNs as compared to unconjugated CRM-SLNs. Thus, TX and Lf crossconjugated SLNs enhance the BBB permeability of the drug with improved antiproliferative action against GBM [39].

\subsubsection{PEG conjugated solid lipid nanoparticles for gene delivery}

Treatment of brain tumor through siRNA is preferable, as it can target specifically to one gene and is able to silence it in a post-transcriptional way. Moreover, siRNA can target several functional proteins available at the BBB [40]. Treatment of brain tumor through siRNA, needs a safe, stable, effective carrier which must be able to cross the BBB. The SLNs are mostly preferred as it meets most of the criteria which siRNA needs. Targeted delivery of gene by SLNs is a bi-stage system. Conjugation of angiopep to SLN surface for targeting the low-density lipoprotein receptor-related protein-1(expressed in BBB) is the initial step. The proteolytic cleavage of PEGylated lipopeptide, which releases PEG, glutamic acid residues and release of siRNA for high silencing efficiency is the second stage [5].

siRNA encapsulated SLNs were successfully developed using a combination of titrable cationic lipids. For effective gene delivery, it was PEGylated after 
incorporating MMP-cleavable lipopeptide. The in vitro study indicated that the developed SLNs showed higher uptake and gene knockdown efficacy. SLNs also showed low cytotoxicity which was owing to masking of intrinsic positive charge of SLNs by PEGylated cleavable lipopeptide. In in vivo studies, angiopep functionalization played a vital role as a mediator of transport across the BBB and targeting to glioma [41].

\subsubsection{Apolipoprotein E conjugated solid lipid nanoparticles}

Low density lipoprotein (LDL) receptors are overexpressed on the BBB and apolipoprotein E (AP-E), a plasmatic protein is easily recognized by these receptors. Resveratrol (RSV), polyphenolic flavonoid promises to offer neuroprotective effects which are helpful in neurological disorders like Alzheimer's, Parkinson's, Huntington's diseases, brain ischemia, and epilepsy. The AP-E conjugated resveratrol (RSV) SLNs were successfully developed. The binding of ApoE to the SLNs surface was carried out by spontaneous interaction between the previously biotinylated ApoE and the covalently attached avidin on the SLNs surface, resulting

\begin{tabular}{|c|c|c|c|c|c|c|c|c|}
\hline $\begin{array}{l}\text { Sl. } \\
\text { no }\end{array}$ & $\begin{array}{l}\text { SLN } \\
\text { (Type) }\end{array}$ & $\begin{array}{l}\text { Lipid } \\
\text { (s) }\end{array}$ & $\begin{array}{l}\text { Preparation } \\
\text { method }\end{array}$ & Drugs & Target & Model & Comments & Ref. \\
\hline 01 & SLN & $\begin{array}{l}\text { CP, } \\
\text { DMPC }\end{array}$ & $\begin{array}{l}\text { High shear } \\
\text { homogenization } \\
\text { and } \\
\text { ultrasonication } \\
\text { techniques }\end{array}$ & CMP & Brain & $\begin{array}{l}\text { Human } \\
\text { glioma \& } \\
\text { Monocytic } \\
\text { cell line. } \\
\text { Wistar rats }\end{array}$ & $\begin{array}{l}\text { Enhanced } \\
\text { accumulation of } \\
\text { CMP. Superior } \\
\text { in vitro antitumor } \\
\text { activity }\end{array}$ & {$[33]$} \\
\hline 02 & SLN & $\begin{array}{l}\text { COMP, } \\
\text { SPC }\end{array}$ & $\begin{array}{l}\text { Warm oil-in- } \\
\text { water } \\
\text { microemulsion } \\
\text { technique }\end{array}$ & RLZ & Brain & Male SD rats & $\begin{array}{l}\text { Higher } \\
\text { bioaccumulation } \\
\text { of RLZ in brain. }\end{array}$ & [34] \\
\hline 03 & SLN & $\begin{array}{l}\text { SA, } \\
\text { GMS, } \\
\text { PRE, } \\
\text { GTP }\end{array}$ & $\begin{array}{l}\text { High shear } \\
\text { homogenization } \\
\text { and } \\
\text { ultrasonication } \\
\text { techniques }\end{array}$ & VIN & Brain & - & $\begin{array}{l}\text { Zero-order } \\
\text { sustained drug } \\
\text { release kinetics }\end{array}$ & {$[35]$} \\
\hline 04 & Lf-SLN & $\begin{array}{l}\text { GMS, } \\
\text { SA, SL }\end{array}$ & $\begin{array}{l}\text { Emulsification } \\
\text { and solvent } \\
\text { evaporation } \\
\text { method }\end{array}$ & DTX & $\begin{array}{l}\text { Lf } \\
\text { Receptors }\end{array}$ & $\begin{array}{l}\text { U-87 MG } \\
\text { cell lines \& } \\
\text { Swiss albino } \\
\text { mice }\end{array}$ & $\begin{array}{l}\text { Improved the } \\
\text { brain targeting } \\
\text { potential }\end{array}$ & [37] \\
\hline 05 & $\begin{array}{l}\text { Tf-Lf- } \\
\text { SLNs }\end{array}$ & $\begin{array}{l}\text { TPM, } \\
\text { DSPE }\end{array}$ & $\begin{array}{l}\text { Homogenization } \\
\text { followed by } \\
\text { centrifugation }\end{array}$ & CRM & $\begin{array}{l}\text { Tf and Lf } \\
\text { Receptors }\end{array}$ & $\begin{array}{l}\text { U87MG, } \\
\text { HBMECs }\end{array}$ & $\begin{array}{l}\text { Higher BBB } \\
\text { permeability. } \\
\text { Superior anti- } \\
\text { proliferative } \\
\text { action }\end{array}$ & [39] \\
\hline 06 & SLN & $\begin{array}{l}\text { POPC, } \\
\text { DSPE, } \\
\text { CHO, } \\
\text { DM }\end{array}$ & $\begin{array}{l}\text { Detergent } \\
\text { dialysis } \\
\text { technique }\end{array}$ & siRNA & Angiopep & $\begin{array}{l}\text { bEnd. } 3 \text { cell, } \\
\text { U87MG } \\
\text { cells, }\end{array}$ & $\begin{array}{l}\text { Higher uptake } \\
\text { and gene } \\
\text { knockdown } \\
\text { efficacy }\end{array}$ & [41] \\
\hline 07 & $\begin{array}{l}\text { AP-E - } \\
\text { SLNs }\end{array}$ & $\mathrm{CP}$ & $\begin{array}{l}\text { High shear } \\
\text { homogenization } \\
\text { followed by } \\
\text { sonication } \\
\text { technique }\end{array}$ & RSV & $\begin{array}{l}\text { LDL } \\
\text { Receptor }\end{array}$ & $\begin{array}{l}\text { hCMEC/D3 } \\
\text { Cell line }\end{array}$ & $\begin{array}{l}\text { Brain targeted } \\
\text { delivery of RSV }\end{array}$ & {$[42]$} \\
\hline
\end{tabular}

Table 2.

List of SLNs and their different ligand conjugated forms for brain cell targeting. 
in two different ApoE-functionalized SLNs: SLN-DSPE-ApoE and SLN-PalmitateApoE. These conjugated SLNs were sufficiently stable and were able to prevail over the issues of RSV like low solubility, degradation but also to help its brain targeted delivery. Brain targeted delivery of RSV by such SLNs follows a bi-stage system. The AP-E-RSV-SLNs mimic lipoprotein particles that are endocytosed into the BBB endothelium via the LDL receptor and then transcytosed to the brain [42].

List of SLNs and their different ligand conjugated forms for brain cell targeting have been summarized in Table 2 .

\section{Solid lipid nanoparticles for liver targeting}

Though targeted delivery of a drug to the liver is a challenging task, still, it is an interesting approach in the treatment of various liver disorders. In the treatment of liver disorders, drugs targeting to the liver, face irresistible obstacles from various physiological barriers and processes like uptake by the reticuloendothelial system, mechanical entrapment by the pulmonary vascular bed, and opsonization process [43].

Numerous approaches are being proposed to enhance bioaccumulation/ biodistribution of drugs to liver and hepatocytes. These approaches include both active targeting as well as passive accumulations of nanoparticulate formulation due to ligand (carbohydrate, peptide, antibodies conjugation) conjugated nanoparticles. Recently, liver targeted deliveries of drugs by the SLNs are gaining much attention in the treatment of various types of liver disorders. Thus, various liver targeting strategies using SLNs are enlightened below.

\subsection{Solid lipid nanoparticles for active targeting}

Baicalin (BCL), a natural product obtained from Scutellaria baicalensis (Family: Labiatae) popularly used in the treatment of Hepatitis-B and liver fibrosis. BCL loaded SLNs were developed. The prepared SLNs were stable and were able to enhance the therapeutic efficacy of BCL by improving its biodistribution in the liver. In vivo biodistribution, targeting evaluation and in vitro anti-oxidant study reveals that the developed BCL-SLNs have substantial liver targeting, improved anti oxidative and hydroxyl radical scavenging abilities [44].

Ficus benjamina (Family: Moraceae) is rich in phenolic (chlorogenic, pcoumaric, ferulic and syringic acids) and total flavonoid content that are effective against chronic alcoholism induced fatal liver and cardio-renal injury. Ethanolic extract of Ficus benjamina (FB) loaded in SLNs (FBSLNs) helped in bioaccumulation of the phenolic and flavonoid content in the liver due to lipophilic nature of SLNs. In vivo evaluation of FBSLNs against hepatic and cardio-renal injury revealed its hepatoprotective activity which was further evident from various biological parameters and histopathological photomicrography. In the liver, accumulation of aldehyde level was reduced that validated the detoxifying nature of FBSLN. Moreover, restoration of aberrant cardio-renal biomarkers and histological consequences revealed the cardio-renal protective potential of FBSLNs [45].

Berberine (BBR), an active constituent of Coptis chinensis (Family: Ranunculaceae) have potential pharmacological effects on type-2 diabetes. It has already been validated that BBR enhances glucose and lipid metabolism through the activation of adenosine monophosphate-activated protein kinase (AMPK) and improve insulin sensitivity. BBR loaded SLNs (BBR-SLNs) were developed to improve the beneficial effect of BBR on hepatosteatosis. The effect of BBR-SLNs on lipid metabolism were studied which revealed gaining of body weight and reduction 
in liver weight with simultaneous reduction of serum alanine transaminase and liver triglyceride level. Biodistribution study reported 20 -fold increase in the concentration of drug in the liver than that of blood. Moreover, it reduced the accumulation of fat and lipid droplet size. It was also noticed that the expression of lipogenic genes was down-regulated and lipolytic gene was up-regulated in BBR-SLNs treated livers which could be helpful in the treatment of hepatosteatosis [46].

Cisplatin (CSPT) is an anti-cancer drug which is used in the treatment of many malignancies including hepatocellular carcinoma, lungs carcinoma, etc. The CSPT loaded SLNs (CSPT-SLNs) were successfully developed and were stable in terms of drug content after storage for 3 months in different temperature and humid conditions. In vivo tissue distribution study revealed that the developed CSPT-SLNs were able to deliver a higher amount of CSPT particularly to the liver as compared to the brain, lungs, and kidney [47].

Sorafenib (SFB), a potent multi-kinase inhibitor possess anti-tumor angiogenesis effect (block vascular endothelial growth factor (VEGF) and platelet-derived growth factor receptor (PDGFR)) and is preferentially used in the treatment of hepatocellular carcinoma. The SFB loaded SLNs (SFB-SLNs) were developed with an objective of improving bioavailability and reducing adverse effects. The results of the stability test showed that SRF-SLNs remained stable for more than 1 month at room temperature. In vivo study of SFB-SLNs revealed improved bioavailability (increased by $66.7 \%$ ) with remarkably higher bioaccumulation of drug in the liver (2.20-fold higher drug selectivity index value) when compared with the SFB suspension [48].

Primaquine phosphate (PP) is an antimalarial drug that acts on the primary tissue forms of the Plasmodium which after growth within the liver, initiate the erythrocytic stage. Thus, PP loaded SLNs (PP-SLNs) were developed with an aim to deliver liver schizonticide PP directly to the hepatocytes. Stability of the PP-SLNs in suspension was tested for a period of 3 months in terms of size, poly-dispersity, $\zeta$-potential, and $\mathrm{pH}$. There were no noteworthy changes in size, poly-dispersity, $\zeta$-potential, or $\mathbf{p H}$ occurred over time. In vivo study report revealed that the developed SLNs were highly effective (>20\%) against hypnozoites/liver stage of all malarial species with a reduced dose when compared with the conventional oral dose [49].

\subsection{Solid lipid nanoparticles delivering gene}

The fibrous scars occurring in the liver due to the increased production and deposition of hepatic extracellular matrix (ECM) components are called liver fibrosis reduce the physiological performance of the liver. Hepatitis viral infection is one of the major reasons for liver fibrosis and cirrhosis. Administration of antifibrotic therapeutics (e.g. connective tissue growth factor (siRNA) responsible for the cellular and molecular basis of fibrogenesis) is one of the most preferable approaches for the treatment of liver fibrosis. The siRNA loaded cationic SLNs (cSLNs) were developed by gently mixing CSLNs with siRNA at various weight ratios of cSLN to siRNA in 0.1 M PBS ( $\mathrm{pH} \mathrm{7.4)} \mathrm{and} \mathrm{then} \mathrm{incubated} \mathrm{at} \mathrm{room} \mathrm{temperature} \mathrm{for} 15 \mathrm{~min}$. Naturally obtained low-density lipids (LDLs) were used in the preparation. The developed cSLN were able to silence the targeted gene in the presence of serum with notably low cytotoxicity. The cSLNs were PEGylated which were hydrodynamically stable and were able to protect their siRNA cargo from nuclease degradation during systemic circulation. The developed cSLNs loaded with siRNA administer through intravenous route delivered siRNA exclusively to the liver and resulted in a considerable reduction in collagen content and pro-fibrogenic factors with spectacular progress of pathophysiological symptoms in a liver fibrosis rat 
model. Biodistribution study revealed site-specific delivery and accumulation of siRNA loaded cSLNs to the liver tissues [50].

\subsection{Solid lipid nanoparticles for passive targeting}

\subsubsection{Polyethylene glycol conjugated (PEGylated) solid lipid nanoparticles for passive targeting}

PEGylated SLNs are reported to be preferentially accumulated in the liver as the kidney is unable to clear the same. Thus, in liver disorders, liver targeting strategy using PEGylation technique is used for delivering numerous drugs. Paclitaxel (PTX) loaded PEGylated SLNs were successfully developed for targeting the liver, in the case of hepatic carcinoma. The cellular uptake study revealed that PTX loaded PEGylated SLNs showed prolonged circulation time in plasma and higher bioaccumulation of drug in the liver when compared with the PTX solution [51].

The preferential drug targeting ability of PEGylated SLNs to cancer cells have been shown in Figure 2.

\subsubsection{Galactosylated lipid [N-hexadecyl lactobionamide] conjugated solid lipid nanoparticles}

The parenchymal cells of the liver contain asialoglycoprotein receptors which recognize terminal b-D-galactose or $\mathrm{N}$-acetylgalactosamine residues. The $\mathrm{N}$ hexadecyl lactobionamide (N-HLBA) was synthesized via an amide bond between the amine group of hexadecylamine and the carboxyl group of lactobionic acid. The

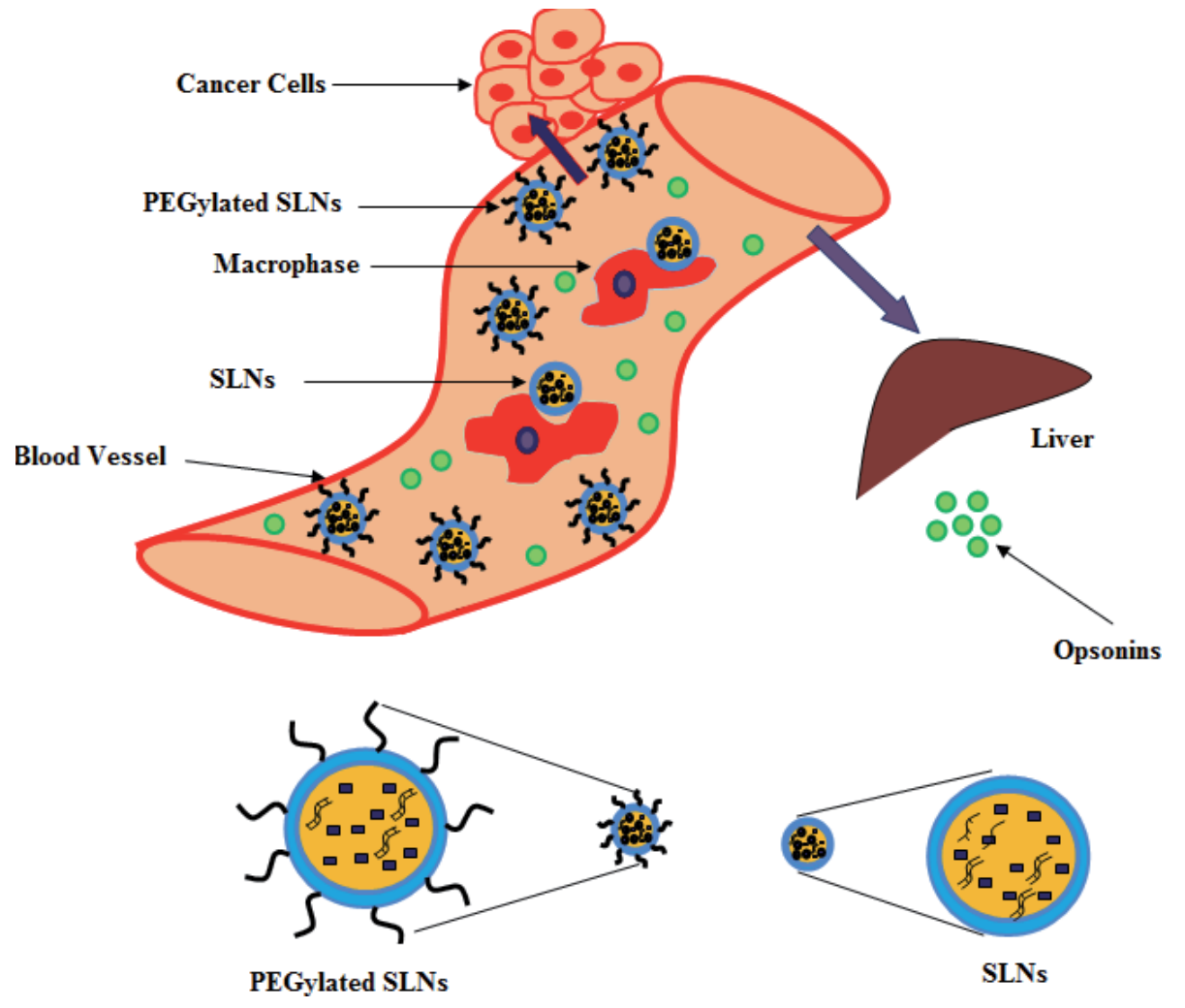

Figure 2.

PEGylated SLN in targeting preferentially to cancer cells. 
lactobionic acid was converted to 1,5-lactone that contain more reactive amine groups. Cucurbitacin B (CurB), a tetracyclic triterpene shows significant pharmacological activities including anti-tumor, anti-hepatitis, hepatocurative and hepatoprotective. The CurB loaded N-hexadecyl lactobionamide (N-HLBA) conjugated SLNs were developed for liver-targeted delivery of CurB. The N-HLBA SLN with anchored galactose moiety via amide bonds might achieve effective livertargeting delivery in vivo by retaining the surface galactose in blood stream, and by exposing a higher amount of galactose to the liver parenchymal cells. The optimum zeta potential held up the physical stability whereas the optimum particle size distribution offered the convenience for intravenous administration and deep penetration into targeting area. The Biodistribution study of the CurB loaded Nhexadecyl lactobionamide (N-HLBA) conjugated SLNs revealed a 2.5-fold increase

\begin{tabular}{|c|c|c|c|c|c|c|c|c|}
\hline $\begin{array}{l}\text { Sl. } \\
\text { no }\end{array}$ & $\begin{array}{l}\text { SLN } \\
\text { (Type) }\end{array}$ & $\begin{array}{l}\text { Lipid } \\
(s)\end{array}$ & $\begin{array}{l}\text { Preparation } \\
\text { method }\end{array}$ & Drugs & Target & Model & Comments & Ref. \\
\hline 01 & SLN & $\begin{array}{l}\text { SL, } \\
\text { GMS }\end{array}$ & $\begin{array}{l}\text { Emulsification } \\
\text { ultrasonic } \\
\text { dispersion } \\
\text { method }\end{array}$ & BCL & Liver & Rats & $\begin{array}{l}\text { Improved } \\
\text { biodistribution of BCL } \\
\text { in Liver, Superior anti } \\
\text { oxidative and } \\
\text { hydroxyl radical } \\
\text { scavenging abilities }\end{array}$ & [44] \\
\hline 02 & SLN & SA & $\begin{array}{l}\text { Hot- } \\
\text { homogenization } \\
\text { followed by } \\
\text { ultra-sonication }\end{array}$ & FB & Liver & $\begin{array}{l}\text { HepG2 cell } \\
\text { line, Rats }\end{array}$ & $\begin{array}{l}\text { Anti-oxidant, anti- } \\
\text { inflammatory and } \\
\text { detoxification } \\
\text { potential }\end{array}$ & {$[45]$} \\
\hline 03 & SLN & $\begin{array}{l}\text { GL, } \\
\text { SP, TP }\end{array}$ & $\begin{array}{l}\text { Hot } \\
\text { Emulsification } \\
\text { Technique }\end{array}$ & BBR & Liver & $\begin{array}{l}\text { Male } \mathrm{db} / \mathrm{db} \\
\text { mice, }\end{array}$ & $\begin{array}{l}\text { Down regulate the } \\
\text { lipogenic gene and } \\
\text { Up-regulate the } \\
\text { lipolytic gene. }\end{array}$ & {$[46]$} \\
\hline 04 & SLN & SA & $\begin{array}{l}\text { Warm } \\
\text { emulsification } \\
\text { followed by } \\
\text { sonication }\end{array}$ & CSPT & $\begin{array}{l}\text { Hepatocellular } \\
\text { carcinomas }\end{array}$ & Wistar rats & $\begin{array}{l}\text { Higher } \\
\text { bioaccumulation of } \\
\text { drug in liver }\end{array}$ & {$[47]$} \\
\hline 05 & SLN & GB & $\begin{array}{l}\text { High-speed } \\
\text { shearing } \\
\text { followed by } \\
\text { ultrasonication }\end{array}$ & SFB & Liver & $\begin{array}{l}\text { Female SD } \\
\text { Rats }\end{array}$ & $\begin{array}{l}\text { Improved } \\
\text { bioavailability, higher } \\
\text { bioaccumulation in } \\
\text { liver }\end{array}$ & {$[48]$} \\
\hline 06 & SLN & SA & $\begin{array}{l}\text { Modified } \\
\text { multiple } \\
\text { emulsion } \\
\text { solvent } \\
\text { evaporation } \\
\text { technique }\end{array}$ & PP & Hepatocytes & 3D7, Mice & $\begin{array}{l}\text { Improved antimalarial } \\
\text { activity }\end{array}$ & [49] \\
\hline 07 & C-SLN & $\mathrm{CO}$ & $\begin{array}{l}\text { Modified } \\
\text { emulsification } \\
\text { and solvent } \\
\text { evaporation } \\
\text { method }\end{array}$ & siRNA & LDL Receptor & $\begin{array}{l}\text { Rat, HSCs } \\
\text { and } \\
\text { hepatocytes }\end{array}$ & $\begin{array}{l}\text { Spectacular progress } \\
\text { of pathophysiological } \\
\text { symptoms in liver } \\
\text { fibrosis }\end{array}$ & {$[50]$} \\
\hline 08 & $\begin{array}{l}\text { PEG- } \\
\text { SLN }\end{array}$ & $\begin{array}{l}\mathrm{GT} \\
\mathrm{CHO}\end{array}$ & $\begin{array}{l}\text { Solvent- } \\
\text { emulsification } \\
\text { method }\end{array}$ & PTX & Hepatocytes & $\begin{array}{l}\text { HepG2, } \\
\text { MCF7, } \\
\text { PANC-1 }\end{array}$ & $\begin{array}{l}\text { Higher liver } \\
\text { bioaccumulation of } \\
\text { PTX }\end{array}$ & {$[51]$} \\
\hline 09 & G-SLN & COMP & $\begin{array}{l}\text { High-pressure } \\
\text { homogenization }\end{array}$ & CurB & $\begin{array}{l}\text { ASGP } \\
\text { Receptor }\end{array}$ & Wistar rats & $\begin{array}{l}\text { Enhanced antitumor } \\
\text { and hepatoprotective } \\
\text { activity }\end{array}$ & {$[52]$} \\
\hline
\end{tabular}

Table 3.

List of SLNs and their different ligand conjugated forms for liver cell targeting. 
in the amount of CurB in the liver when compared with CurB-SLN. In vitro cytotoxicity study revealed enhancement of cytotoxicity. The experimental result validated the liver targeting ability of N-HLBA conjugated SLNs [52].

List of SLNs and their different ligand conjugated form for liver targeting have been summarized in Table 3.

\section{Solid lipid nanoparticles for breast cell targeting}

Breast cancer is the most common form of cancer and the second most deadly disease among the woman around the globe. The breast cancer new incidences and mortality rate has been increased by 20 and 14\% since 2008 .

Recently, controlled release of the drugs to the targeted site of the disease using a nanocarrier vehicle is getting more attention as it enhances the therapeutic efficacy of the drugs. Solid lipid nanoparticulate (SLN) formulations possess an endless potential to deliver active chemotherapeutic molecules in a programmed prototype to improve bioavailability and nullify the side-effects. The bio-compatibility and bio-degradability characteristics of SLNs promise to offer a lesser toxic product as compared to polymeric nanoparticles which forced to consider it as an idealistic targeted drug delivery system for breast cancer therapy [5].

\subsection{Solid lipid nanoparticle for active breast cell targeting}

\subsubsection{SLNs for photodynamic therapy}

Photodynamic therapy is one of the emerging approaches in the treatment of cancer which comprises application of a photosensitizer followed by laser irradiation of tumor lesions. Temoporfin (TP), a photosensitizer loaded in thermoresponsive SLNs were developed with an objective to improve anticancer activity through site specific drug delivery. The copolymer poly(ethylene oxide)-blockpoly ( $\varepsilon$-caprolactone) copolymers $\left(\mathrm{PEO}_{45}-\mathrm{b}-\mathrm{PCL}_{7}\right)$ were synthesized by the mechanism of catalyst-free ring opening polymerization of $\varepsilon$-caprolactone which was initiated by poly(ethylene oxide) monomethyl ether(MPEO). These copolymers were acts as a stabilizer in the preparation of thermoresponsive SLN. The stability study report revealed that the developed SLNs had higher stability in human serum within the blood transport to tumor tissue. In vitro phototoxicity study revealed higher phototoxic activity of TP against breast cancer cells due to its faster bioaccumulation in the targeted cancer cells. The in vivo anticancer efficacy of TPSLN was remarkably higher as compared to the commercial TP formulation [53].

\subsubsection{Manganese (Mn) II complex solid lipid nanoparticles}

Transition metal complex (e.g., Manganese II complex $\left.\left[\mathrm{Mn}_{2}\left(\mu\left(\mathrm{C}_{6} \mathrm{H}_{5}\right)_{2} \mathrm{CHCOO}\right)_{2}(\text { bipy })_{4}\right]^{ \pm 2}\right)$ has been extensively used for cancer therapy nowadays due to its potential anticancer activity (interact with the DNA). Mn(II) complex loaded SLNs were developed that showed superior cytotoxicity activity on breast cancer cells. The zeta potential value of the product was higher indicating good physical stability and dispersion quality of the SLNs. Cell proliferation assay revealed that the normal cell death rate was lower with Mn(II) SLNs which indicated lesser toxicity of the product to the normal cell. Moreover, higher early apoptosis rate was observed with Mn(II) complex SLN as compared to Mn(II) alone [54]. 


\subsection{Solid lipid nanoparticles for passive breast cell targeting}

\subsubsection{Fucose conjugated solid lipid nanoparticles}

Fucose receptors are overexpressed in the breast cancer cell. Thus, conjugation of fucose to SLNs was proposed to deliver the drug specifically to breast cancerous cells. Fucose conjugated methotrexate (MTX) loaded SLNs were developed to achieve enhanced targeting potential for breast cancer cells. Fucosylation of MTXSLNs was related with opening of fucose ring and reaction of its aldehyde group with free amino functionalities expressed over the surface of MTX-SLNs in sodium acetate buffer ( $\mathrm{pH} 4.0$ ). The above process led to the formation of Schiff's base ($\mathrm{N}=\mathrm{CH})$. The Schiff's base might be reduced to secondary amine $\left(-\mathrm{NHCH}_{2}\right)$ and establish equilibrium with Schiff's base. Physical stability of prepared SLNs was higher which could be due to positive zeta potential value that provides repulsive interaction between nanosized lipid particles preventing particle aggregation. The ex vivo study revealed higher cellular uptake as well as higher cytotoxicity at lower IC50 of MTX. The in vitro study results showed increased rate apoptosis with a change in lysosomal membrane permeability and a higher rate of lysosomal membrane degradation. The in vivo study revealed maximum bioavailability and tumor targeting efficiency with minimum secondary drug distribution to other organs [55].

\subsubsection{Folic acid functionalized solid lipid nanoparticles}

The folate receptor (FR) is one of the most widely evaluated receptor for active targeting of anticancer therapeutics in the case of in breast cancer cells. Folic acid has many advantages over antibody ligands such as small size, nonimmunogenicity, non-toxicity, ease of handling, stability and low cost [56]. Several researchers had reported earlier that the FA functionalized SLNs were able to deliver the chemotherapeutic agent, particularly to the cancerous cells. Thus, FA functionalized SLNs co-encapsulated with Docetaxel (DTX) and Curcumin (CUR) were successfully developed to enhance its therapeutic efficacy against breast cancer cells. FA-stearic acid (FA-SA) conjugate was synthesized by classical 1-ethyl-3(3-dimethylaminopropyl) carbodiimide (EDAC) chemistry and incorporated in the DTX-CUR-SLN. Additionally, PEG-stearic acid (PEG-SA) was incorporated to obtain FA-DTX-CUR-SLN. The DTX-CUR-SLN and FA-DTX-CUR-SLN formulations were found to be stable at refrigerated condition for 2 months. Both the formulations showed sign of instability at accelerated condition $\left(25^{\circ} \mathrm{C} / 65 \% \mathrm{RH}\right)$. The developed FA functionalized SLNs exhibited improved pharmacokinetic parameters, superior cancer cell targeting efficiency, and improved therapeutic efficacy of DTX. Folic acid was believed to be responsible for the targeting efficacy of the developed SLNs to the breast cancer cell. This conjugated system showed significant increase in area under the curve and mean residence time of the drug. Co-conjugation (FA and PEG) to SLNs co-encapsulated with DTX and CUR responsible for synergistic activity of both DTX and CUR. Moreover, bioaccumulation of DTX in heart and kidney was found very low which signified avoidance of vital organ toxicity [57].

\subsubsection{Cetamide and trimethylphytosphingosine-iodide co-conjugated solid lipid nanoparticles}

The sphingosine-1-phosphate (S1P), phytosphingosine, ceramides, and sphingosine are the metabolites of sphingolipids and are reported as essential structural 
components of cell membranes or important mediators of cellular process that regulate the proliferation, survival, and death of cells. Moreover, sphingosines including N,N,N-trimethylsphingosine (TMP-I) have been reported for their role as a negative modulator of transmembrane signaling through protein kinase $C$ (PKC) as well as an inhibitor of sphingosine kinase-1 (SK-1), controlling the various membrane-associated signaling mechanisms associated with cell growth and inhibitory apoptosis in tumor cells [58]. Ceramide, a kind of sphingosine conjugated with fatty acid residue, is also reported to enhance the sensitivity of MDR-acquired cancer cell lines to chemotherapeutic agents [59]. Thus, attempts were made to employ ceramide (CD) and trimethylphytosphingosine-iodide (TMP-I) as a targeting agent for docetaxel (DTX) loaded SLNs. The prepared SLNs were physically stable without any significant change in their physical appearance, drug content, and particle size over a period of 8 weeks at $4^{\circ} \mathrm{C}$. CD enhanced the DTX sensitivity in MDR-acquired cancer cell lines. In vivo clearance of drug and tumor growth inhibitors were significantly decreased in case of CD and TMP-I conjugated SLNs when compared with the marketed product. Thus, CD and TMP-I conjugated DTX-SLN could serve as a potentials alternative parenteral formulations of DTX [60].

\begin{tabular}{|c|c|c|c|c|c|c|c|c|}
\hline $\begin{array}{l}\text { Sl. } \\
\text { no }\end{array}$ & $\begin{array}{l}\text { SLN } \\
\text { (Type) }\end{array}$ & Lipid(s) & Drugs & $\begin{array}{l}\text { Preparation } \\
\text { method }\end{array}$ & Target & Model & Comments & Ref. \\
\hline 01 & SLN & $\mathrm{TD}$ & TMP & $\begin{array}{l}\text { Modified hot } \\
\text { homogenization } \\
\text { and } \\
\text { ultrasonication } \\
\text { method }\end{array}$ & $\begin{array}{l}\text { Breast } \\
\text { Cancer } \\
\text { cell }\end{array}$ & $\begin{array}{l}4 \mathrm{~T} 1 \text {, } \\
\text { MDA-MB-231, } \\
\text { Female } \mathrm{Nu} / \mathrm{Nu} \\
\text { mice- MDA- } \\
\text { MB-231 }\end{array}$ & $\begin{array}{l}\text { Exhibited } \\
\text { improved } \\
\text { phototoxicity and } \\
\text { anticancer } \\
\text { efficacy }\end{array}$ & [53] \\
\hline 02 & $\begin{array}{l}\text { Mn (II) } \\
\text { Complex- } \\
\text { SLN }\end{array}$ & COMP & $\begin{array}{l}\text { Mn (II) } \\
\text { complex }\end{array}$ & $\begin{array}{l}\text { Hot } \\
\text { homogenization } \\
\text { method }\end{array}$ & $\begin{array}{l}\text { Breast } \\
\text { Cancer } \\
\text { cell }\end{array}$ & $\begin{array}{l}\text { MCF-7 and } \\
\text { HUVEC cell } \\
\text { line }\end{array}$ & $\begin{array}{l}\text { Possessed } \\
\text { superior } \\
\text { anticancer } \\
\text { activity with } \\
\text { reduced toxic } \\
\text { effect. }\end{array}$ & [54] \\
\hline 03 & F-SLN & $\begin{array}{l}\text { PL90NG, } \\
\text { PL, STA, } \\
\text { GEL }\end{array}$ & MTX & $\begin{array}{l}\text { Hot } \\
\text { microemulsion } \\
\text { method }\end{array}$ & $\begin{array}{l}\text { Fucose } \\
\text { Receptor }\end{array}$ & $\begin{array}{l}\text { MCF-7 Cell } \\
\text { line \& Female } \\
\text { SD rat }\end{array}$ & $\begin{array}{l}\text { Improved } \\
\text { bioavailability } \\
\text { and tumor } \\
\text { targeting } \\
\text { efficiency with } \\
\text { minimum } \\
\text { secondary drug } \\
\text { distribution in } \\
\text { various organs. }\end{array}$ & [55] \\
\hline 04 & $\begin{array}{l}\text { FA-PEG- } \\
\text { SLN }\end{array}$ & GMS, SA & $\begin{array}{l}\text { CUR, } \\
\text { DTX }\end{array}$ & $\begin{array}{l}\text { Modified } \\
\text { ethanol injection } \\
\text { method }\end{array}$ & $\begin{array}{l}\text { Folic } \\
\text { acid } \\
\text { Receptor }\end{array}$ & $\begin{array}{l}\text { MCF-7 } \\
\text { \&MDA-MB- } \\
\text { 231Cell line. } \\
\text { Female Wistar } \\
\text { Rat }\end{array}$ & $\begin{array}{l}\text { Synergistic } \\
\text { cancer efficacy } \\
\text { due to } \\
\text { coencapsulation } \\
\text { of DTX and CRM } \\
\text { along with } \\
\text { targeted } \\
\text { delivery of drugs }\end{array}$ & [57] \\
\hline 05 & $\begin{array}{l}\text { CD-TMP } \\
\text { I-SLN }\end{array}$ & PC, TMS, & DTX & $\begin{array}{l}\text { High-pressure } \\
\text { homogenization } \\
\text { method }\end{array}$ & $\begin{array}{l}\text { CD \& } \\
\text { TMP-1 }\end{array}$ & $\begin{array}{l}\text { MCF-7 cell, } \\
\text { MCF-7/ADR } \\
\text { cells }\end{array}$ & $\begin{array}{l}\text { Significant } \\
\text { increased } \\
\text { antitumor } \\
\text { efficacy with } \\
\text { targeted drug } \\
\text { delivery }\end{array}$ & [60] \\
\hline
\end{tabular}

Table 4.

List of SLNs and their different ligand conjugated forms for breast cancer cell targeting. 
List of SLNs and their different ligand conjugated form for breast cancer cell targeting have been summarized in Table 4.

\section{Solid lipid nanoparticles for eye targeting}

The eye is one of the delicate organs of human and one of the most delicate routes of drug delivery. However, the eye poses unique challenges relative to drug delivery due to the ocular anatomical and physiological constraints. SLNs are one of the promising targeted DDS for an eye. Numerous drugs such as antibiotics, plasmids, anti-inflammatory, and immunosuppressive agents were encapsulated in SLNs for the treatment of ophthalmic disorders.

\subsection{Solid lipid nanoparticles for active eye targeting}

Corneal neovascularization (CNV), a sight-threatening condition is caused due to various inflammatory settings including chemical injury. Single-stranded proline-modified short hairpin anti-angiopoietin-like protein 2 (ANGPTL2 RNA) interference molecules acts as potent angiogenic and pro-inflammatory factor and is used for the treatment of CNV [61]. Thus, ANGPTL2 RNA loaded SLNs were developed to deliver the interference molecule specifically to the retina. The singlestranded RNAi (pshRNA) loaded SLNs exhibit high stability in vivo and preliminary data suggest that pshRNA is less susceptible to degeneration due to RNase activity. The fluorescence-labeled developed SLNs eye drops penetrated all layers of cornea and expression of ANGPTL2mRNA was considerably inhibited in both epithelium and stroma. Further, the area of angiogenesis was remarkably decreased in the corneas by the SLNs drops as compared to the control group [62].

$\mathrm{X}$-linked juvenile retinoschisis (XJR), a retinal degenerative disorder caused by mutation in the RS1 gene encoding a retinoschisin [63]. Among non-viral vectors, solid lipid nanoparticles (SLNs) represent one of the most effective lipid-based colloidal carriers, and for gene delivery to the posterior segment of the eye [64]. Plasmid (human RS1 gene) loaded SLNs were developed for the treatment of XJR diseases which showed significant improvement of the retinal structure with photoreceptor specific expression of the RS1 gene [65].

Tobramycin (TMC) is one of the most preferable drugs to treat vitreoretinal diseases, such as bacterial infections, endophthalmitis, cytomegalovirus retinitis (CMV), uveitis, proliferative vitreoretinopathy (PVR), diabetic retinopathy, agerelated macular degeneration [66]. Thus TMC loaded mucoadhesive SLNs were developed which showed higher bioaccumulation of drugs in most of the ocular tissues and was able to penetrate into the retina. Moreover, it resulted in enhanced intraphagocytic antibiotic concentration in polymorphonuclear granulocytes and superior bactericidal activity against Pseudomonas aeruginosa. The developed SLNs showed good stability up to 12 months with no aggregation or precipitation phenomena, variations in zeta potential values or in amount of the drug encapsulated into the nanoparticles [67].

\subsection{Solid lipid nanoparticles for passive eye targeting}

\subsubsection{Chitosan coated solid lipid nanoparticles}

Indomethacin (IMC), a topical non-steroidal anti-inflammatory drug (NSAID) is used for ocular inflammatory disorders such as conjunctivitis, uveitis, cystoid macular edema, and anterior segment inflammation, including post-operative pain 
following cataract surgery [68]. Chitosan (Cs) coated IMC loaded SLNs (IMC-CsSLN) were developed to deliver NSAID to the posterior segment of ocular tissues and for improving the pre-corneal residence time and transcorneal permeability characteristics. For surface modification of the developed SLNs, the chitosan was incorporated into the aqueous phase prior to preparation of the SLNs. The developed SLNs were stable in terms of drug loading, EE, and less drug expulsion during storage at $40^{\circ} \mathrm{C}$ for 90 days. The SLNs showed higher bioaccumulation of IMC in the ocular tissues. The IMC-Cs-SLN showed superior trans-membrane IMC permeation characteristics which were due to penetration enhancing properties of Cs [69].

\subsubsection{Intercalated montmorillonite solid lipid nanoparticles}

Betaxolol hydrochloride (BH) is widely used for the treatment of ocular hypertension and open-angle glaucoma in clinical therapeutics. However, it faces certain limitations like low bioavailability and pre-ocular retention, and some side effects.

\begin{tabular}{|c|c|c|c|c|c|c|c|c|}
\hline $\begin{array}{l}\text { Sl. } \\
\text { no }\end{array}$ & SLN (Type) & $\operatorname{Lipid}(s)$ & Drugs & $\begin{array}{l}\text { Preparation } \\
\text { method }\end{array}$ & Target & Model & Comments & Ref. \\
\hline 01 & SLN & $\begin{array}{l}\text { DSGPC, } \\
\mathrm{CHO}\end{array}$ & $\begin{array}{l}\text { ANGPTL2 } \\
\text { RNA }\end{array}$ & $\begin{array}{l}\text { Hydration } \\
\text { method } \\
\text { followed by } \\
\text { extrusion }\end{array}$ & Retina & $\begin{array}{l}\text { C57BL/6 } \\
\text { mice }\end{array}$ & $\begin{array}{l}\text { Inhibition of } \\
\text { expression of } \\
\text { ANGPTL2mRNA, } \\
\text { Reduction in } \\
\text { angiogenesis area }\end{array}$ & {$[62]$} \\
\hline 02 & SLN & PRE & $\begin{array}{l}\text { Human RS1 } \\
\text { gene }\end{array}$ & $\begin{array}{l}\text { Solvent } \\
\text { emulsification } \\
\text { followed by } \\
\text { evaporation }\end{array}$ & Retina & $\begin{array}{l}661 \mathrm{~W}, \\
\text { RS1h- } \\
\text { deficient } \\
\text { mouse }\end{array}$ & $\begin{array}{l}\text { Improvement of } \\
\text { the retinal } \\
\text { structure }\end{array}$ & {$[65]$} \\
\hline 03 & SLN & SA & TMC & $\begin{array}{l}\text { Warm o/w } \\
\text { microemulsion } \\
\text { method }\end{array}$ & $\begin{array}{l}\text { Aqueous } \\
\text { humor }\end{array}$ & $\begin{array}{l}\text { Albino } \\
\text { rabbit }\end{array}$ & $\begin{array}{l}\text { Superior } \\
\text { bactericidal } \\
\text { activity }\end{array}$ & {$[67]$} \\
\hline 04 & Cs-SLN & GB & IMC & $\begin{array}{l}\text { Hot } \\
\text { homogenization }\end{array}$ & $\begin{array}{l}\text { Posterior } \\
\text { segment of } \\
\text { ocular } \\
\text { tissue }\end{array}$ & $\begin{array}{l}\text { White } \\
\text { albino } \\
\text { Rabbits }\end{array}$ & $\begin{array}{l}\text { Improved } \\
\text { biodistribution of } \\
\text { IMC at posterior } \\
\text { segment }\end{array}$ & [69] \\
\hline 05 & Mt-SLNs & $\begin{array}{l}\text { PC, } \\
\text { GMS }\end{array}$ & $\mathrm{BH}$ & $\begin{array}{l}\text { Emulsion } \\
\text { evaporation- } \\
\text { low } \\
\text { temperature } \\
\text { solidification } \\
\text { method }\end{array}$ & $\begin{array}{l}\text { Cornea and } \\
\text { Conjunctiva }\end{array}$ & Rabbit & $\begin{array}{l}\text { Significantly } \\
\text { reduced } \\
\text { inflammation, No } \\
\text { irritation }\end{array}$ & [70] \\
\hline 06 & PEG-SLNs & COMP & KTZ & $\begin{array}{l}\text { Emulsification } \\
\text { followed by } \\
\text { high pressure } \\
\text { homogenizer }\end{array}$ & $\begin{array}{l}\text { Upper } \\
\text { posterior } \\
\text { eye }\end{array}$ & $\begin{array}{l}\text { ARPE-19 } \\
\text { \& RCE } \\
\text { Cell line, } \\
\text { Rat }\end{array}$ & $\begin{array}{l}\text { Superior } \\
\text { antifungal activity }\end{array}$ & {$[73]$} \\
\hline 07 & $\begin{array}{l}\text { Multifunctional } \\
\text { SLNs }\end{array}$ & $\mathrm{CP}$ & $\begin{array}{l}\text { BAI (Drug) } \\
\text { C IR-780 } \\
\text { (Diagnostic } \\
\text { agent) }\end{array}$ & $\begin{array}{l}\text { Modified } \\
\text { solvent- } \\
\text { diffusion } \\
\text { method }\end{array}$ & $\begin{array}{l}\text { Colorectal } \\
\text { part }\end{array}$ & $\begin{array}{l}\text { LoVo, } \\
\text { CHO-K1 }\end{array}$ & $\begin{array}{l}\text { Imaging, Superior } \\
\text { cytotoxicity }\end{array}$ & {$[75]$} \\
\hline 08 & $\begin{array}{l}\text { SIA-PEGylated } \\
\text { SLN }\end{array}$ & $\begin{array}{l}\text { GMS, } \\
\text { OA }\end{array}$ & DXM & $\begin{array}{l}\text { Solvent } \\
\text { diffusion } \\
\text { method }\end{array}$ & $\begin{array}{l}\text { E-selectin } \\
\text { receptor }\end{array}$ & $\begin{array}{l}\text { HUVECs, } \\
\text { ICR male } \\
\text { Mice }\end{array}$ & $\begin{array}{l}\text { Targeted delivery } \\
\text { of DXM for } \\
\text { ischemia- } \\
\text { reperfusion- } \\
\text { induced injury- } \\
\text { induced AKI }\end{array}$ & {$[78]$} \\
\hline
\end{tabular}

Table 5 .

List of SLNs and their different ligand conjugated forms for eye, colon and kidney targeting. 
In order to overcome these limitations acid treated montmorillonite (Mt)- Betaxolol Hydrochloride (BH) nanocomposite encapsulated SLNs (Mt-BH-SLNs) were developed. An acid-treated montmorillonite (acid-Mt) was first intercalated with $\mathrm{BH}$ in the interlayers and this nanocomposite was encapsulated by SLNs. The developed Mt-BH-SLNs possess good stability. Long term irritation test reported that the (Mt-BH-SLNs) showed no damage for cornea and conjunctiva. The corneal hydration level of Mt-BH-SLNs was higher (78.25 \pm 0.63$) \%$ indicating higher drug corneal permeability and absence of irritation to the cornea. Thus, Mt-BH-SLNs could be used for effective management of glaucoma [70].

\subsubsection{Polyethylene glycol (PEG) conjugated (PEGylated) solid lipid nanoparticles}

Ketoconazole (KTZ) is a broad spectrum antifungal agent, with high liposolubility [71] but a short ocular half-life (elimination half-life is $19 \mathrm{~min}$ in aqueous humor and $43 \mathrm{~min}$ in cornea) [72] and very poor solubility $(0.04 \mathrm{mg} / \mathrm{ml})$. Ketoconazole (KTZ) loaded PEGylated SLNs were developed for targeted delivery of KTZ to the posterior part of the eye for treatment of fungal infection. It showed higher bioavailability both in the aqueous and vitreous humor with significant antifungal potential. The ex vivo corneal permeation study revealed higher corneal permeability of the PEGylated KTZ-SLNs. The developed SLN was satisfying various parameters suitable for ocular delivery such as $\mathrm{pH}$, osmolarity, stability, autoclavability, particle size, preservation against contamination. The SLNs were found to be stable in terms of entrapment efficiency and total drug content at $2-8^{\circ} \mathrm{C}$ for 12 months. Thus it could be helpful in the treatment of keratitis and endophthalmitis [73].

List of SLNs and their different ligand conjugated forms for eye targeting have been summarized in Table 5 .

\section{Solid lipid nanoparticles for passive colon targeting}

Combination of nanocarriers and electroporation techniques is named as electropermeabilization which is commonly used for enhancing drug transport. The Cyanine-type IR 780 and Baicalein (BAI) co-encapsulated SLNs were developed for both imaging and therapy of colorectal carcinoma where cyanine-type IR 780 and baicalein (flavonoid derivative) were acting as a diagnostic agent (photosensitizer) and therapeutic cargo respectively. For preparation of SLNs the organic phase was prepared by dissolving IR-780, BAI, and melted lipid in dichloromethane. The organic phase was then added dropwise to hot aqueous phase containing surfactant under vigorous stirring. Supplementary material (flavonoids) facilitated in the reduction of dose and reduction in normal cell toxicity in cancer chemotherapy. The external electric field pulses applied in electroporation helped in increased of cell membrane permeability, either by generating transient pores or membrane electropermeabilization [74]. Electropermeabilization mediated administration of the developed SLNs showed cytoskeletal abnormalities more significantly then without electropermeabilization. The prepared SLNs particles were with good physical stability. With electroporation support, the developed SLNs showed increased p53 and manganese superoxide dismutase expression with significant higher cytotoxicity, thus validating their suitability for combined therapy and molecular imaging simultaneously [75].

List of SLNs and their different ligand conjugated form for colon targeting have been summarized in Table 5 . 


\section{Solid lipid nanoparticles for kidney targeting}

\subsection{Solid lipid nanoparticles for passive kidney targeting}

\subsubsection{Polyethylene glycol (PEG) surface modified solid lipid nanoparticles}

Icariin (IRN) is widely used as traditional Chinese medicine for the treatment of kidney diseases and reinforce yang. The PEG surface modified Icariin (IRN) loaded SLNs (PEG-IRN-SLNs) was developed for targeted delivery of IRN to the kidney and to improve the bioavailability. The SLN was prepared by high temperature melt-cool solidification method. Upon comparing with IRN solution it was revealed from the pharmacokinetic study that the biological half-life $\left(t_{1 / 2}\right)$ and area under curve (AUC) of PEG-IRN-SLN was 7-fold and 4-fold higher. Biodistribution study revealed that IRN concentration in kidney tissues was significantly increased. Moreover, the relative target efficiency to kidney tissues was $79 \%$ and relative tissue exposure was 16.95. Thus the develop SLN could be helpful in the treatment of kidney diseases [76].

\subsubsection{Sialic acid conjugated PEGylated solid lipid nanoparticles}

E-selectin is a promising target for the site-specific delivery of antiinflammatory agents. Several researchers have reported that sialic acid (SA)-mediated micelles could be specifically internalized by lipopolysaccharide (LPS)-activated human umbilical vein endothelial cells (HUVECs) via the specific binding between SA and E-selectin receptor [77]. Sialic acid (SIA) conjugated PEGylated dexamethasone (DXM) loaded SLNs (SIA-PEGylated-DXM-SLN) were developed to deliver DXM specifically to the kidney and to improve the therapeutic efficacy of DXM for renal ischemia-reperfusion injury (IRI)-induced acute renal injury. The Sialic acid (SIA) conjugated PEGylated DXM (SIA-PEG-DXM) was synthesized by adding PEG-DXM, dicyclohexylcarbodiimide (DCC), and 4-dimethylaminopyridine (DMAP) into anhydrous dimethyl formamide (DMF) followed by addition of SIA into the solution. The resulting mixture was stirred for obtaining SIA-PEGDXM. The crude product was purified by dialysis against deionized water for 2 days, followed by lyophilization. The developed SLNs potentially had good colloidal stability in human body. The study revealed that the apoptotic human umbilical vein endothelial cells (HUVECs) were significantly decreased. It indicated the suitability of SIA-PEGylated-SLNs for internalization by the inflamed vascular endothelial cells. Biodistribution study revealed higher renal accumulation of DXM (range 2.7- to 5.88-fold higher) after $6 \mathrm{~h}$ of intravenous administration. The Pharmacodynamic study revealed that higher blood biochemical indexes, histopathological changes, oxidative stress levels, and pro-inflammatory cytokines which indicated improved renal function by the influence of SIA-PEGylated DXM [64].

List of SLNs and their different ligand conjugated form targeting to the kidney have been summarized in Table 5 .

\section{Conclusion}

Though drug targeting to a specific site in the body is an interesting approach, it is a highly challenging task. Despite that a large variety of smart nanocarriers have been developed for drug targeting in recent years, SLN has achieved a special status among them and can be employed for both passive as well as active targeting. These can be 
employed for delivering not only the drug but also antibody, proteins, genes, imaging agents etc. and bring about increased cellular uptake at the targeted disease sites. Moreover, surface modification of SLNs through conjugation of various ligands on SLNs surfaces helped in enhancing its targeting efficacy in terms of cellular binding, uptake and intracellular transport to different cells as well as organs and ensure its greatest potentiality to combat wide ranges of diseases. However, the intrinsic complexity of biological environments strongly influences its functionality and often complicates their effective use for therapeutic treatments. Therefore, a deeper knowledge and understanding of the real interactions involved in the diseased tissues is fundamental for the development of therapeutic protocols of SLNs.

\section{Conflict of interest}

The authors confirm that this article content has no conflicts of interest.

\section{Abbreviations}

\begin{tabular}{|c|c|}
\hline AM & alveolar macrophages \\
\hline ANGPTL2 RNA & $\begin{array}{l}\text { single-stranded proline-modified short hairpin anti- } \\
\text { angiopoietin-like protein } 2\end{array}$ \\
\hline AP-E & apolipoprotein $\mathrm{E}$ \\
\hline ASGP & asialoglycoprotein \\
\hline BAI & baicalein \\
\hline BBR & berberine \\
\hline BCL & baicalin \\
\hline $\mathrm{BH}$ & betaxolol hydrochloride \\
\hline $\mathrm{CHO}$ & cholesterol \\
\hline CIR-780 & cyanine-type IR-780 \\
\hline CMP & camptothecin \\
\hline $\mathrm{CO}$ & cholesterol oleate \\
\hline COMP & compritol 888 ATO \\
\hline $\mathrm{CP}$ & cetyl palmitate \\
\hline CRM & carmustine \\
\hline c-SLN & cationic SLN \\
\hline CSPT & cisplatin \\
\hline Cs-SLN & chitosan coated SLNs \\
\hline CUR & curcumin \\
\hline CurB & curcumin B \\
\hline $\mathrm{DM}$ & dimyristoyl \\
\hline DMPC & 1,2-dimyristoyl-sn-glycero-3-phospho-choline \\
\hline DSPE & Distearoylphosphatidyl-ethanolamine \\
\hline DSPEG & $\begin{array}{l}\text { 1,2-distearoyl-snglycero-3-phosphoethanolamine- } \mathrm{N} \text {-[carboxy } \\
\text { (polyethylene glycol)-2000] }\end{array}$ \\
\hline DSGPC & 1,2-distearoyl-sn-glycero-3-phosphocholine \\
\hline DTX & docetaxel \\
\hline DXM & dexamethasone \\
\hline FA-PEG-SLN & folic acid-polyethylene glycol cross conjugated SLN \\
\hline FB & Ficus benjamina \\
\hline FR & folate receptor \\
\hline F-SLN & fucose conjugated SLN \\
\hline Ft-SLN & folate conjugated SLN \\
\hline GB & glyceryl behenate \\
\hline
\end{tabular}




\begin{tabular}{|c|c|}
\hline $\begin{array}{l}\text { GEL } \\
\text { GL }\end{array}$ & $\begin{array}{l}\text { Gelucire }{ }^{\circledR 50 / 13} \\
\text { glycerol }\end{array}$ \\
\hline GMS & glvcervl monostearate \\
\hline GS & glyceryl stearate \\
\hline G-SLNs & galactosylated lipid conjugated SLNs \\
\hline GT & glycerol trioleate \\
\hline GTP & glyceryl tripalmitate \\
\hline GTS & Glycerol tristearate \\
\hline HSC & hepatic stellate cells \\
\hline IMC & indomethacin \\
\hline LDL & low density lipid \\
\hline LfR & lactoferrin receptor \\
\hline Lf-SLN & lactoferrin SLN \\
\hline KTZ & ketoconazole \\
\hline MIC & minimum inhibitory concentration \\
\hline Mn (II) complex & $\left.\left[\mathrm{Mn}_{2}\left(\mathrm{l}\left(\mathrm{C}_{6} \mathrm{H}_{5}\right)_{2} \mathrm{CHCOO}\right)_{2} \text { (bipy }\right)_{4}\right]$ (bipy) $\left(\mathrm{ClO}_{4}\right)_{2}$ complex \\
\hline MR & mannose receptor \\
\hline Msy-SLN & mannosylated SLN \\
\hline Mt. & intercalated montmorillonite SLN \\
\hline MTX & methotrexate \\
\hline $\mathrm{PA}$ & palmitic acid \\
\hline PC & phosphatidylcholine \\
\hline PEG-SLN & PEGylated SLNs \\
\hline PL & phospholipid \\
\hline PL90NG & Phospholipon 90NG \\
\hline PP & primaquine phosphate \\
\hline PRE & Precirol ATO5 \\
\hline POPC & 1-palmitoyl-2-oleoyl-sn-glycero-3-phosphocholine \\
\hline PTX & paclitaxel \\
\hline RIF & rifampicin \\
\hline RFB & rifabutin \\
\hline RSV & resveratrol \\
\hline RLZ & riluzole \\
\hline SA & stearic acid \\
\hline SFB & sorafenib \\
\hline SL & soya lecithin \\
\hline SIA & sialic acid \\
\hline siRNA & small interfering RNA \\
\hline $\mathrm{SD}$ & Sprague-Dawley \\
\hline SP & soybean phospholipid \\
\hline SPC & soya phosphatidylcholine \\
\hline STA & stearyl amine \\
\hline $\mathrm{TD}$ & 1-tetradecanol \\
\hline TMC & tobramycin \\
\hline TMP & temoporfin \\
\hline TMP-I & trimethylphytosphingosine-iodide \\
\hline TMS & trimyristin \\
\hline TP & tripalmitate \\
\hline TSN & tristearin \\
\hline VIN & vinpocetine \\
\hline WGA-SLN & wheat germ agglutinin conjugated SLN \\
\hline 3D7 & asexual intraerythrocytic stage of $P$. falciparum 1 \\
\hline
\end{tabular}




\section{Author details}

Bibhash Chandra Mohanta ${ }^{1}$, Subas Chandra Dinda ${ }^{2 *}$, Narahari Narayan Palei ${ }^{3}$ and Jyotirmoy Deb ${ }^{4}$

1 Seemanta Institute of Pharmaceutical Sciences, Jharpokharia, Mayurbhanj, Odisha, India

2 College of Pharmacy, Teerthanker Mahaveer University, Moradabad, Uttar Pradesh, India

3 Sree Vidyanikethan College of Pharmacy, Tirupati, Andhra Pradesh, India

4 Department of Pharmacy, HUDA Group of Institution, Nagaon, Assam, India

*Address all correspondence to: subas.dinda@rediffmail.com

\section{IntechOpen}

(C) 2019 The Author(s). Licensee IntechOpen. This chapter is distributed under the terms of the Creative Commons Attribution License (http://creativecommons.org/licenses/ by/3.0), which permits unrestricted use, distribution, and reproduction in any medium, provided the original work is properly cited. (c) BY 


\section{References}

[1] Himri I, Guaadaoui A. Cell and organ drug targeting: Types of drug delivery systems and advanced targeting strategies. In: Grumezescu AM, editor. Nanostructures for the Engineering of Cells, Tissues and Organs: From Design to Applications. Oxford, United Kingdom: Willium Andrew Applied Science Publisher (an imprint of Elsevier); 2018. pp. 1-66. DOI: $10.1016 /$ B978-0-12-813665-2.00001-6

[2] Saltzman WM, Torchilin VP. Drug delivery systems. In: Access Science. 16th ed. Chicago: McGraw-Hill Education; 2016. DOI: $10.1036 /$ 1097-8542.757275

[3] Singh R, Willard JJW. Nanoparticlebased targeted drug delivery. Experimental and Molecular Pathology. 2009;86:215-223. DOI: $10.1016 / \mathrm{j}$. yexmp.2008.12.004

[4] Bibhas CM, Subas CD, Gitanjali M, Narahari NP. Exploring the use of lipid based nano-formulations for the management of tuberculosis. Journal of Nanosciences: Current Research. 2017; 2(3):1-15. DOI: 10.4172/2572-0813. 1000112

[5] Palei NP, Mohanta BC, Sabapathi ML, Das MK. Lipid-based nanoparticles for cancer diagnosis and therapy. In: Grumezescu AM, editor. Organic Materials as Smart

Nanocarriers for Drug Delivery. Oxford, United Kingdom: Willium Andrew Applied Science Publisher (an imprint of Elsevier); 2018. pp. 415-470. DOI: 10.1016/B978-0-12-813663-8.00010-5

[6] Sharma J, Kalra S, Sharma A, Rani S. Colloidal drug carriers. The Internet Journal of Family Practice. 2009; 9(2):1-6

[7] Maiti S, Sen KK. Drug delivery concepts. In: Maiti S, Sen KK, editors. Advanced Technology for Delivering
Therapeutics. Rijeka, Croatia: IntechOpen; 2016. pp. 1-13. DOI: 10.5772/65245

[8] Rostami E, Kashanian S, Azandaryani AH, Faramarzi H, Dolatabadi JEN, Omidfar K. Drug targeting using solid lipid nanoparticles. Chemistry and Physics of Lipids. 2014; 181:56-61. DOI: 10.1016/j.chemphyslip. 2014.03.006

[9] Deshpande A, Mohamed M, Daftardar SM, Patel M, Boddu SHS, Nesamony J. Solid lipid nanoparticles in drug delivery: Opportunities and challenges. In: Mitra A, Cholkar K, Mandal A, editors. Emerging Nanotechnologies for Diagnostics, Drug Delivery and Medical Devices Micro and Nano Technologies. Amsterdam, Netherlands: Elsevier; 2017. pp. 291-330. DOI: 10.1016/B978-0323-42978-8.00012-7

[10] Dhanasekaran S, Chopra S. Getting a handle on smart drug delivery systems-A comprehensive view of therapeutic targeting strategies. In: Sezer AD, editor. Smart Drug Delivery System. Rijeka, Croatia: IntechOpen; 2015. pp. 31-62. DOI: 10.5772/61388

[11] Mansour HM, Rhee YS, Wu X. Nanomedicine in pulmonary delivery. International Journal of Nanomedicine. 2009;4:299-319. DOI: 10.2147/IJN.S4937

[12] Liu J, Gong T, Fu H, et al. Solid lipid nanoparticles for pulmonary delivery of insulin. International Journal of Pharmaceutics. 2008;356(1-2):333-344.

DOI: 10.1016/j.ijpharm.2008.01.008

[13] Bi R, Shao W, Wang Q, Zhang N. Solid lipid nanoparticles as insulin inhalation carriers for enhanced pulmonary delivery. Journal of Biomedical Nanotechnology. 2009;5: 84-92. DOI: $10.1166 /$ jbn.2009.036 
[14] Chuan J, Li Y, Yang L, Sun X, Zhang Q, Gong T, et al. Enhanced rifampicin delivery to alveolar macrophages by solid lipid nanoparticles. Journal of Nanoparticle Research. 2013;15:1634-1641

[15] Gospar DP, Faria V, Goncalves LMD, Taboada $\mathrm{P}$, Remuñán-López $\mathrm{C}$, Almeida AJ. Rifabutin-loaded solid lipid nanoparticles for inhaled antitubercular therapy: Physicochemical and in vitro studies. International Journal of Pharmaceutics. 2016;497: 199-209

[16] Singh H, Bhandari R, Kaur IP. Encapsulation of rifampicin in a solid lipid nanoparticulate system to limit its degradation and interaction with isoniazid at acidic $\mathrm{pH}$. International Journal of Pharmaceutics. 2013;446: 106-111

[17] Xiang QY, Wang MT, Chen F, Gong T, Jian YL, Zhang ZR, et al. Lungtargeting delivery of dexamethasone acetate loaded solid lipid nanoparticles. Archives of Pharmacal Research. 2007; 30(4):519-525

[18] Varshosaz S, Ghaffari SF, Mirshojaei A, Jafarian F, Atyabi F, Kobarfard SA. Biodistribution of amikacin solid lipid nanoparticles after pulmonary delivery. BioMed Research International. 2013;2013:1-8. DOI: $10.1155 / 2013 / 136859$

[19] Muro S. Challenges in design and characterization of ligand-targeted drug delivery systems. Journal of Controlled Release. 2012;164(2):125-137. DOI: 10.1016/j.jconrel.2012.05.052

[20] Elfinger M, Maucksch C, Rudolph C. Characterization of lactoferrin as a targeting ligand for nonviral gene delivery to airway epithelial cells. Biomaterials. 2007;28: 3448-3455. DOI: 10.1016/j.biomaterials. 2007.04.011
[21] Shilpi S, Vimal VD, Soni V. Assessment of lactoferrin-conjugated solid lipid nanoparticles for efficient targeting to the lung. Progress in Biomaterials. 2015;4:55-63. DOI: 10.1007/s40204-015-0037-z

[22] Gorelik E, Galili U, Raz A. On the role of cell surface carbohydrates and their binding proteins (lectins) in tumor metastasis. Cancer Metastasis Reviews. 2001;20:245-277. DOI: 10.1023/A: 101553542759

[23] Ertl B, Franziska H, Wirth M, et al. Lectin-mediated bioadhesion: Preparation, stability and Caco-2 binding of wheat germ agglutininfunctionalized poly(D,L-lactide-coglycolic acid)-microspheres. Journal of Drug Targeting. 2000;8:173-184. DOI: $10.3109 / 10611860008996863$

[24] Coelho LCBB, Silva PMDS, Lima VLDM, Pontual EV, Paiva PMG, Napoleão TH, et al. Lectins, interconnecting proteins with biotechnological/pharmacological and therapeutic applications. Evidencebased Complementary and Alternative Medicine. 2017;2017:1-22. DOI: 10.1155/ 2017/1594074

[25] Pooja D, Tunki L, Kulhari H, Reddy BB, Sistla R. Characterization, biorecognitive activity and stability of WGA grafted lipid nanostructures for the controlled delivery of Rifampicin. Chemistry and Physics of Lipids. 2015; 193:11-17. DOI: $10.1016 /$ j.chemphyslip. 2015.09.008

[26] Lane KB, Egan B, Vick S, Abdolrasulnia R, Shepherd VL. Characterization of a rat alveolar macrophage cell line that expresses a functional mannose receptor. Journal of Leukocyte Biology. 1998;64(3):345-350. DOI: $10.1002 / \mathrm{jlb} .64 .3 .345$

[27] Nimje N, Agarwal A, Saraogi GK, Lariya N, Rai G, Agrawal H, et al. Mannosylated nanoparticulate carriers 
of rifabutin for alveolar targeting. Journal of Drug Targeting. 2009; 17(10):777-787. DOI: 10.3109/ 10611860903115308

[28] Sahu PK, Mishra DK, Jain N, Rajoriya V, Jain AK. Mannosylated solid lipid nanoparticles for lung-targeted delivery of paclitaxel. Drug Development and Industrial Pharmacy. 2015;41(4):640-649. DOI: 10.3109/ 03639045.2014 .891130

[29] Shi H, Guo J, Li C, Wang Z. A current review of folate receptor alpha as a potential tumor target in non-smallcell lung cancer. Drug Design, Development and Therapy. 2015;9: 4989-4996. DOI: 10.2147/DDDT.S90670

[30] Rosière R, Van Woensel M, Gelbcke M, Mathieu V, et al. New folate-grafted chitosan derivative to improve delivery of paclitaxel-loaded solid lipid nanoparticles for lung tumor therapy by inhalation. Molecular Pharmaceutics. 2018;15(3):899-910. DOI: $10.1021 /$ acs.molpharmaceut. $7 \mathrm{~b} 00846$

[31] Brioschi AM, Calderoni S, Zara GP, Priano L, Gasco MR, Mauro A. Solid lipid nanoparticles for brain tumors therapy: State of the art and novel challenges. In: Sharma HS, editor. Progress in Brain Research. Amsterdam, Netherlands: Elsevier; 2009. pp. 193-223. DOI: $10.1016 /$ S0079-6123 (08)80011-8

[32] Kuo YC, Liang CT. Inhibition of human brain malignant glioblastoma cells using carmustine-loaded catanionic solid lipid nanoparticles with surface antiepithelial growth factor receptor. Biomaterials. 2011;32: 3340-3350

[33] Martins SM, Sarmento B, Nunes C, et al. Brain targeting effect of camptothecin-loaded solid lipid nanoparticles in rat after intravenous administration. European Journal of
Pharmaceutics and Biopharmaceutics. 2013;85:488-502. DOI: 10.1016/j. ejpb.2013.08.011

[34] Bondì ML, Craparo EF, Giammona G, Drago F. Brain-targeted solid lipid nanoparticles containing riluzole: Preparation, characterization and biodistribution. Nanomedicine. 2010;5(1):25-32. DOI: $10.2217 /$ nnm.09.67

[35] Morsi NM, Ghorab DM, Badie HA. Brain targeted solid lipid nanoparticles for brain ischemia: Preparation and in vitro characterization.

Pharmaceutical Development and Technology. 2013;18(3):736-744. DOI: $10.3109 / 10837450.2012 .734513$

[36] Fillebeen C, Descamps L, Dehouck MP, Fenart L, Benaïssa M, Spik G, et al. Receptor-mediated transcytosis of lactoferrin through the blood-brain barrier. The Journal of Biological Chemistry. 1999;274: 7011-7017. DOI: 10.1074/jbc.274.11.7011

[37] Singh I, Swami R, Pooja D, et al. Lactoferrin bioconjugated solid lipid nanoparticles: A new drug delivery system for potential brain targeting. Journal of Drug Targeting. 2016;24(3): 212-223. DOI: 10.3109/1061186X.2015. 1068320

[38] Lee WL, Cheng MH, Chao HT, Wang $\mathrm{PH}$. The role of selective estrogen receptor modulators on breast cancer: From tamoxifen to raloxifene.

Taiwanese Journal of Obstetrics \& Gynecology. 2008;47(1):24-31. DOI: $10.1016 / \mathrm{S} 1028-4559(08) 60051-0$

[39] Kuo YC, Cheng SJ. Brain targeted delivery of carmustine using solid lipid nanoparticles modified with tamoxifen and lactoferrin for antitumor proliferation. International Journal of Pharmaceutics. 2016;499: 10-19. DOI: 10.1016/j.ijpharm.2015. 12.054 
[40] Gomes MJ, Martins S, Sarmento B. siRNA as a tool to improve the treatment of brain diseases: Mechanism, targets and delivery. Ageing Research Reviews. 2015;21:43-54. DOI: 10.1016/j. arr.2015.03.001

[41] Brunn J, Larsen TB, Jolck RI, et al. Investigation of enzyme-sensitive lipid nanoparticles for delivery of siRNA to blood-brain barrier. International Journal of Nanomedicine. 2015;10: 5995-6008. DOI: 10.2147/IJN.S87334

[42] Neves AR, Queiroz JF, Reis S. Braintargeted delivery of resveratrol using solid lipid nanoparticles functionalized with apolipoprotein E. Journal of Nanbiotechnology. 2016;14(27):1-11. DOI: $10.1186 / \mathrm{s} 12951-016-0177-\mathrm{x}$

[43] Mishra N, Yadav NP, Rai VK, Sinha P, Yadav KS, Jain S, et al. Efficient hepatic delivery of drugs: Novel strategies and their significance. BioMed Research International. 2013;2013:1-20. DOI: $10.1155 / 2013 / 382184$

[44] Yang P, Lian YU, Yan-qiu HU, Lina MA, et al. Study on targeting and in vitro anti-oxidation of baicalin solid lipid nanoparticles. Chinese Herbal Medicines. 2012;4(4):335-339. DOI: 10.3969/j.issn.1674-6348.2012.04.012

[45] Sharma AK, Kumar A, Kumar S, Mukherjee S, et al. Preparation and therapeutic evolution of Ficus benjamina solid lipid nanoparticles against alcohol abuse/antabuse induced hepatotoxicity and cardio-renal injury. RSC Advances. 2017;7:35938-359490. DOI: $10.1039 / C 7 R A 04866 A$

[46] Xue M, Zhang L, Yang M-X, Zhang W. Berberine-loaded solid lipid nanoparticles are concentrated in the liver and ameliorate hepatosteatosis in $\mathrm{db} / \mathrm{db}$ mice. International Journal of Nanomedicine. 2015;10:5049-5057. DOI: 10.2147/IJN.S84565

[47] Doijad RC, Manvi FV, Godhwani DM, Joseph R, Deshmukh NV. Formulation and targeting efficiency of cisplatin engineered solid lipid nanoparticles. Indian Journal of Pharmaceutical Sciences. 2008;70(2):203-207. DOI: 10.4103/0250-474X.41456

[48] Wang H, Wang H, Yang W, et al. Improved Oral bioavailability and liver targeting of sorafenib solid lipid nanoparticles in rats. AAPS PharmSciTech. 2018;19(2):761-768. DOI: 10.1208/s12249-017-0901-3

[49] Owuor JJ, Oloo F, Ouma D, Omwoyo WN, et al. Optimization and characterization of primaquine-loaded solid lipid nanoparticles (SLN) for liver schizonticide targeting by freeze drying. MOJ Drug Design Development \& Therapy. 2017;1(3):104-112

[50] Kong WH, Park K, Lee MY, et al. Cationic solid lipid nanoparticles derived from apolipoprotein-free LDLs for target specific systemic treatment of liver fibrosis. Biomaterials. 2013;34: 542-551. DOI: 10.1016/j.

biomaterials.2012.09.067

[51] Bani-Jaber A, Cui H, Elsaid A, Yalcin M, Sudha T, Mousa SA. Pegylated solid lipid nanoparticles reconstituted from high-density lipoprotein components for hepatic targeting. Science Letters Journal. 2015;4:145

[52] Wang W, Zhao X, Hu H, Chen D, $\mathrm{Gu}$ J, Deng Y, et al. Galactosylated solid lipid nanoparticles with cucurbitacin B improves the liver targetability. Drug Delivery. 2010;17(3): 114-122

[53] Brezaniova I, Hruby M, Kralova J, Kral V, et al. Temoporfin-loaded 1-tetradecanol-based thermoresponsive solid lipid nanoparticles for photodynamic therapy. Journal of Controlled Release. 2016;241:34-44

[54] Guney EG, Cecener G, Dikmen G, Kani I, Egeli U, Tunca B. A novel $\left[\mathrm{Mn}_{2}\left(\mu-\left(\mathrm{C}_{6} \mathrm{H}_{5}\right)_{2} \mathrm{CHCOO}\right)_{2}(\text { bipy })_{4}\right]$ 
(bipy) $\left(\mathrm{ClO}_{4}\right)_{2}$ complex loaded solid lipid nanoparticles: Synthesis, characterization and in vitro cytotoxicity on MCF-7 breast cancer cells. Journal of Microencapsulation. 2016;33(6):575-584. DOI: $10.1080 /$ 02652048.2016 .1228704

[55] Garg NK, Singh B, Jain A, et al. Fucose decorated solid-lipid nanocarriers mediate efficient delivery of methotrexate in breast cancer therapeutics. Colloids and Surfaces. B, Biointerfaces. 2016;146:114-126

[56] Li W Jr, Szoka FC. Lipid-based nanoparticles for nucleic acid delivery. Pharmaceutical Research. 2007;24: 438-449. DOI: 10.1007/s11095-0069180-5

[57] Pawar H, Surapaneni SK, Tikoo K, Singh C, et al. Folic acid functionalized long-circulating co-encapsulated docetaxel and curcumin solid lipid nanoparticles: In vitro evaluation, pharmacokinetic and biodistribution in rats. Drug Delivery. 2016;23(4): 1453-1468

[58] Petremand J, Widmann C. Lipid metabolism: Sphingolipids- from membrane constituents to signaling molecules that control cell-to-cell communications. Current Opinion in Lipidology. 2008;19(6):620-621

[59] Devalapally H, Duan Z, Seiden MV, Amiji MM. Modulation of drug resistance in ovarian adenocarcinoma by enhancing intracellular ceramide using tamoxifen-loaded biodegradable polymeric nanoparticles. Clinical Cancer Research. 2008;14(10):3193-3203

[60] Balakrishnan P, Song CK, Jahu A, Cho HJ. Ceramide and N,N,N trimethyl phytosphingosine-iodide (TMP-I)based lipid nanoparticles for Cancer therapy. Pharmaceutical Research. 2016; 33:206-216

[61] Kanda A, Noda K, Oike Y, Ishida S. Angiopoietin-like protein 2 mediates endotoxin-induced acute inflammation in the eye. Laboratory Investigation. 2012;92:1553-1563. DOI: 10.1038/ labinvest.2012.111

[62] Taketani Y, Usui T, Toyono T, et al. Topical use of angiopoietin-like protein 2 RNAi-loaded lipid nanoparticles suppresses corneal neovascularization. Molecular Therapy-Nucleic Acids. 2016; 5:e292. DOI: 10.1038/mtna.2016.1

[63] Sikkink SK, Biswas S, Parry NR, Stanga PE, Trump D. X-linked retinoschisis: An update. Journal of Medical Genetics. 2007;44:225-232. DOI: $10.1136 / j m g .2006 .047340$

[64] Rodríguez-Gascón A, Solinís MA, dei Pozo-Rodríguez A, Delgado D, Pedraz JL. Lipid nanoparticles for gene therapy. Patent No. US 20120183589 A1; 2012

[65] Apaolaza PS, Rodríguez AP, Solinís MA, et al. Structural recovery of the retina in a retinoschisin-deficient mouse after gene replacement therapy by solid lipid nanoparticles.

Biomaterials. 2016;90:40-49. DOI:

10.1016/j.biomaterials.2016.03.004

[66] Novack GD, Robin AL. Ocular pharmacology. Journal of Clinical Pharmacology. 2016;56:517-527. DOI: 10.1002/jcph.634

[67] Chetonia P, Burgalassia S, Monti D, et al. Solid lipid nanoparticles as promising tool for intraocular tobramycin delivery: Pharmacokinetic studies on rabbits. European Journal of Pharmaceutics and Biopharmaceutics. 2016;109:214-223. DOI: $10.1016 / j$. ejpb.2016.10.006

[68] Toker MI, Erdem H, Erdogan H, Arici MK, Topalkara A, Arslan OS, et al. The effects of topical ketorolac and indomethacin on measles conjunctivitis: Randomized controlled trial. American Journal of Ophthalmology. 2006;141(5):902-905. DOI: $10.1016 / j$. ajo.2005.12.004 
[69] Balguri SP, Adelli GR, Majumdar S. Topical ophthalmic formulations of indomethacin for delivery to the posterior segment ocular tissues. European Journal of Pharmaceutics and Biopharmaceutics. 2016;109:224-235. DOI: 10.1016/j.ejpb.2016.10.015

[70] Hou D, Hu S, Huang Y, et al. Preparation and in vitro study of lipid nanoparticles encapsulating drug loaded montmorillonite for ocular delivery. Applied Clay Science. 2016;119(2): 277-283. DOI: 10.1016/j.clay.2015.10.028

[71] Logua AD, Faddabs AM, Anchisib C, Maccionib AM, Sinicob C, Schivoa ML, et al. Effects of in-vitro activity of miconazole and ketoconazole in phospholipid formulations. The Journal of Antimicrobial Chemotherapy. 1997;40:889-893. DOI: $10.1093 / \mathrm{jac} /$ 40.6 .889

[72] Zhang J, Wang L, Gao C, Zhang L, Xia H. Ocular pharmacokinetics of topically-applied ketoconazole solution containing hydroxypropyl betacyclodextrin to rabbits. Journal of Ocular Pharmacology and Therapeutics. 2008;24:501-506

[73] Kakkar S, Karuppayil SM, Raut JS, et al. Lipid-polyethylene glycol based nano-ocular formulation of ketoconazole. International Journal of Pharmaceutics. 2015;495(1):276-289. DOI: 10.1016/j.ijpharm.2015.08.088

[74] Kotulska M. Electrochemotherapy in cancer treatment. Advances in Clinical and Experimental Medicine. 2007;16(5):601-607

[75] Kulbacka J, Pucek A, Kotulsk M, et al. Electroporation and lipid nanoparticles with cyanine IR-780 and flavonoids as efficient vectors to enhanced drug delivery in colon cancer. Bioelectrochemistry. 2016;110:19-31

[76] Liu K-p, Wang L-f, Li Y, Yang B, Du C, Wang Y. Preparation,
Pharmacokinetics, and tissue distribution properties of icariin-loaded stealth solid lipid nanoparticles in mice. Chinese Herbal Medicines. 2012;4(2): 170-174. DOI: 10.3969/j. issn.1674-6384.2012.02.012

[77] Hu JB, Kang XQ, Liang J. E-selectintargeted sialic acid-PEGdexamethasone micelles for enhanced anti-

inflammatory efficacy for acute kidney injury. Theranostics. 2017;7:2204-2219. DOI: $10.7150 /$ thno. 19571

[78] Hu JB, Song G-L, Liu D, et al. Sialic acid-modified solid lipid nanoparticles as vascular endothelium-targeting carriers for ischemia-reperfusioninduced acute renal injury. Drug Delivery. 2017;24(1):1856-1867. DOI: 10.1080/10717544.2017.1410258 


\title{
Drug Delivery through Targeted Approach with Special References to Phytosomes
}

\author{
Mahendra Rana, Aadesh Kumar and Amita J. Rana
}

\begin{abstract}
Novel drug delivery is a great tool to deliver the drug at a specific site of action by the means of specific drug carrier like Solid-lipid Nanoparticles, Nanostructured lipid carriers, lipid vesicles, liposomes, phytosomes and ethosomes. Every carrier used in novel drug delivery system to deliver the drug at targeted site requires some special formulation techniques. These techniques help the drug carrier to deliver the active drug at targeted site, by reducing the side-effect, minimizing the dose, increasing the absorption and increasing the efficacy of the drug. There is a recent upsurge to move "back to nature" for healing body ailments because the report states that conventional treatment cause various side effects to the human body after prolonged used. Various novel drug delivery vesicles/'somes' are being used for the delivery of phytoconstitutents to the targeted site of action. Phytosome is one of the more reliable and best option for the delivery of herbal constituent to the targeted site. The combination of Novel drug delivery with the transdermal route may be a good idea having fast and targeted delivery of drug. Many health challenges like skin diseases, skin burns, migraine, allergies, cardiac problems, diabetes and trauma like bone fracture could be easily managed by the combination of novel drug delivery and the transdermal route in future.
\end{abstract}

Keywords: novel drug delivery, Phytosomes, herbal products, trauma, lipid vesicles, trandermal route

\section{Background}

With the advent of civilization Human beings have demonstrated their dependence on Nature and plant to fulfill all basic needs. In an eventuality of an infirmity, diseased situation or healing of body ailments the use the plant based remedies had been on the anvil. With the passing of time the knowledge of healing from natural resources developed into science of healing and was documented in India as Ayurveda. The other world event over had similar chronological developments that led to the validation of the traditional knowledge of healing, that strength of of herbal formulations for the treatment of disease and body ailments. Most of the herbal formulations are administered via oral route and are poorly absorbed due to hydrophobic behavior which leads to decreased bioavailability and increased systemic clearance. Repeated administration or dose increment is required to maintain the therapeutic efficacy thereby limiting the use of herbal medicines [1]. Commonly, the herbal medicines utilize crude powders, extracts be formulation 
in accordance to the statutory compendia which might not be needed in large doses, limited route of drug administration but also could be less effective than the contemporary therapeutic formulations. The rapid progress of allopathic drug lead to its dominance in the therapeutic field. The various types of dosage form of allopathic drugs are available to treat the disease conditions i.e. solid dosage form (tablets, capsules, and powders), Liquid dosage form (syrup, suspension, and emulsion given by oral route and injectables given by parental route), and aerosols. Nausea, vomiting, and dizziness are some common side effects associated with these conventional dosage forms of allopathic system. Major complications have come forth after prolonged use of these formulations. There has been a continuous research on reducing side effects experienced by the patients resulting in some advancement to such conventional dosage form. Novel Drug delivery System (NDDS) is one such area of drug research that focuses on the target specific site of action and to minimize the side effects of the conventional dosage form. Nowadays, there has been a change in global trend from synthetic to natural medicine, which we can say "back to nature." The efficiency of medicinal plant species, or herbal medicine, depends on the active molecules present since they provide synergistic action and thus enhance the therapeutic effectiveness. Research is being continuously carried out for the amalgamation of Novel drug delivery and phytoconstituents to ward off any prevalent side effects of NDDS in allopathic system of treatment after prolonged used [2].

There are many kinds of herbal medicines and supplements used worldwide. The global market of phytomedicines currently stands at over $\$ 60$ billion annually. The sale of herbal medicines was expected $6.4 \%$ on an average annual growth rate in 2012 [3, 4] (Table 1).

It is widely accepted that $70-80 \%$ of people globally rely on natural resources for their treatment. In the year 2017 the American Botanical council accepted that the sale of herbal supplements increased by $7.7 \%$ in US (on basis of turnover of herbal market in 2016). Europe leads the chart owing to extensive R\&D for herbal medicine owing to considerable increase in funding for research on herbal medicinal plants. America stands on second position in the global herbal medicine market and held $16.2 \%$ of market share in 2017. The consumption of herbal supplements in US was majorly used for the conditions such as heart stroke (48.7\%), Cancer (43.1\%), and arthritis (43\%) announced by National center for biotechnology information in 2017. Asia Pacific stands on third position having a growth rate of 5.99\% due to the adoption of traditional medicine by pharmaceutical companies, researchers, and policy maker [5].

\begin{tabular}{lcc}
\hline Global herbal market & & \\
\hline Market size & (US\$ billions) & Percentage \\
\hline European Union & 28 & 45 \\
\hline Rest EU & 2.4 & 4 \\
\hline ASEAN & 10.8 & 19 \\
\hline Japan & 9.8 & 16 \\
\hline North America & 6.9 & 11 \\
\hline Others & 4.1 & 7 \\
\hline Total & 60 & 100 \\
\hline Source: http://www.markertresearchfuture.com/reports/herbal-medicine-market-325.
\end{tabular}

Source: http://www.markertresearchfuture.com/reports/herbal-medicine-market-325.

Table 1.

Segmentation of global herbal market in 2012. 
The major players in global herbal medicine market are Arkopharma, Beovita, Hisimo Pharmaceuticals, Bayer AG, Schaper \& Brummer Venus Pharma, and Arizona Natural products. Some key players in the industry involved in the production of herbal medicine and supplements include Bio-Botanica Inc., Dabur Ltd., Guangzhu Pharma Co., Nature's Answer, Inc., Sanjiu Medical \& Pharmaceutical Co. Ltd. The high market demand and an upbeat market trend in favor of natural products are likely to boost the growth in the days to come [5].

Current drug market-mix contains numerous formulations based on medicinal plants and phytoconstituents. However the constant decree that the herbal formulations lack proper standardization protocols and is less efficacious or require larger dose to show its efficacy has deprived the impetus to become the alternative treatment regimen. Application of recent technological advancements such as amalgamation of novel drug delivery with herbal drugs would augment the efficacy of the traditional system.

Various approaches for improving bioavailability of novel herbals formulations followed as under [6-8]:

- Chemical derivatization to improve bioavailability which, however, generates a number of chemical analogs that need to be appropriately screened.

- Combination of the active molecules with other compounds as adjutants promoting the active molecule's absorption.

- Stabilizing natural molecules and promotion of intestinal absorption.

- Improving pharmacokinetic profile by formulating with dietary ingredients (soy lecithin).

Many herbal formulations are being developed with the help of novel technologies of incorporation the phytoconstituents in the vesicles of lipid bi-layers. These vesicles work as novel carriers to deliver the drug at its targeted site. Some advanced drug delivery techniques use novel vehicles "somes" as nanobiomedicine delivered by different route of drug delivery. The novel carrier should ideally fulfill two prerequisites. Firstly, it should deliver the drug at predefined period of time or according to the body requirements. Secondly, it should channel the active constituent to the site of action [9]. There are different types of novel drug vehicle as tabulated, use to deliver the drug molecules on the site of action (Table 2).

The nano-carrier used in novel drug delivery system of herbal drugs has a potential future, improving the activity and overcoming the problem associated with herbal constituents [10]. Any type of medicament could be delivered to its specific site of action with the help of novel drug delivery system. Researchers are

\begin{tabular}{ll}
\hline$\bullet$ Liposomes & $\bullet$ Nanoparticles and microspheres \\
\hline$\bullet$ Niosomes & $\bullet$ Solid lipid nanoparticles \\
\hline - Hydrogel nanoparticles & $\bullet$ Microparticles \\
\hline - Resealed erythrocytes & $\bullet$ Supramolecular biovectors \\
\hline - Lipoproteins & $\bullet$ Cyclodextrins \\
\hline - Polymeric micelles & $\bullet$ Aquasomes \\
\hline - Dendrimers & $\bullet$ Emulsions \\
\hline
\end{tabular}

Table 2.

Different types of novel drug delivery systems [10]. 
currently underway to develop an ideal drug delivery system which satisfies the need of targeted site of action. Biological membrane presents a barrier through which a drug must pass before it gets absorbed or excreted. Lipid solubility and molecular size of drug molecule pose two major limiting factors to pass the biological membrane by which drug can be absorbed systematically following oral or topical administration [11].

The conventional herbal dosage form has a problem of standardization and quality control which had relegated its usage in the in last few decades. When the side effects of allopathic drugs outweigh their risk to usage the focus for treatment shifts towards the natural components and herbal system of treatment. In herbal novel drug development polymeric nanoparticles and nanocapsules like liposomes, solid lipid nanoparticles, phytosomes and nanoemulsion are been formulated. NDDS provides many advantages for herbal drugs including enhancement of solubility and bioavailability, protection from toxicity, enhancement of stability, enhancement of pharmacological action, improving tissue macrophages distribution, sustained delivery, and protection from physical and chemical degradation. The efficacy of herbal product (or medication) is dependent upon effective route of delivery and level of the active compounds [12].

Novel drug delivery systems deliver the drug components at a rate directed by the need of the body during the period of treatment and channelize the active entity onto the site of action. A number of novel drug delivery systems have been used to deliver herbal medicaments encompassing various routes of administration to achieve controlled and targeted drug delivery, for example, encapsulated vesicles can prolong the existence of the drug in systemic circulation and reduce the toxicity if selective uptake can be achieved through drug targeting, sustained or controlled release of conventional medicines [11, 12].

The novel drug delivery vehicles can be classified on the basis of their size, shape, and their composition (Table 3).

\subsection{Liposomes}

Liposomes are micro-sized spheres in which an aqueous core is surrounded by one or more lipid bi-layer. The lipid bi-layers are separated by water or aqueous buffer compartments, liposomes are simple microscopic vesicles in which aqueous volume is entirely enclosed by a membrane composed of lipid bilayers [13] (Figure 1).

\begin{tabular}{lll}
\hline Name of somes & Particle size/shape & Composition \\
\hline Liposomes [13] & $\begin{array}{l}\text { 100-1000 nm(diameter)/ } \\
\text { spherical }\end{array}$ & Phospholipids and cholesterol \\
\hline Resealed erythrocytes [14] & $6-9 \mu \mathrm{m}$ (diameter)/oval & Plasma, protein and platelets \\
\hline Niosomes [15] & $\begin{array}{l}100-140 \mathrm{~nm} \text { (diameter)/ } \\
\text { spherical }\end{array}$ & Non-ionic surfactants and cholesterol \\
\hline Discomes [16] & $16-20 \mu \mathrm{m} /$ disc shaped vesicles & Cholesterol and niosomes \\
\hline Transferosomes [17] & $170-200 \mathrm{~nm} /$ oval & $\begin{array}{l}\text { Soya phophatidylcholine surfactant } \\
\text { and drug/antigen }\end{array}$ \\
\hline Ethosomes [18] & $50-100 \mathrm{~nm} / \mathrm{spherical}$ & Phospholipid and ethanol \\
\hline Phytosomes [19, 20] & $10-100 \mathrm{~nm} /$ spherical & Phospholipid and herbal extract \\
\hline Aquasome/hydrosomes & $60-300 \mathrm{~nm} /$ circular & Ceramics, carbon [21] \\
\hline
\end{tabular}

Table 3.

Classification of herbal novel drug delivery approach on the basis of their size, shape, and composition. 


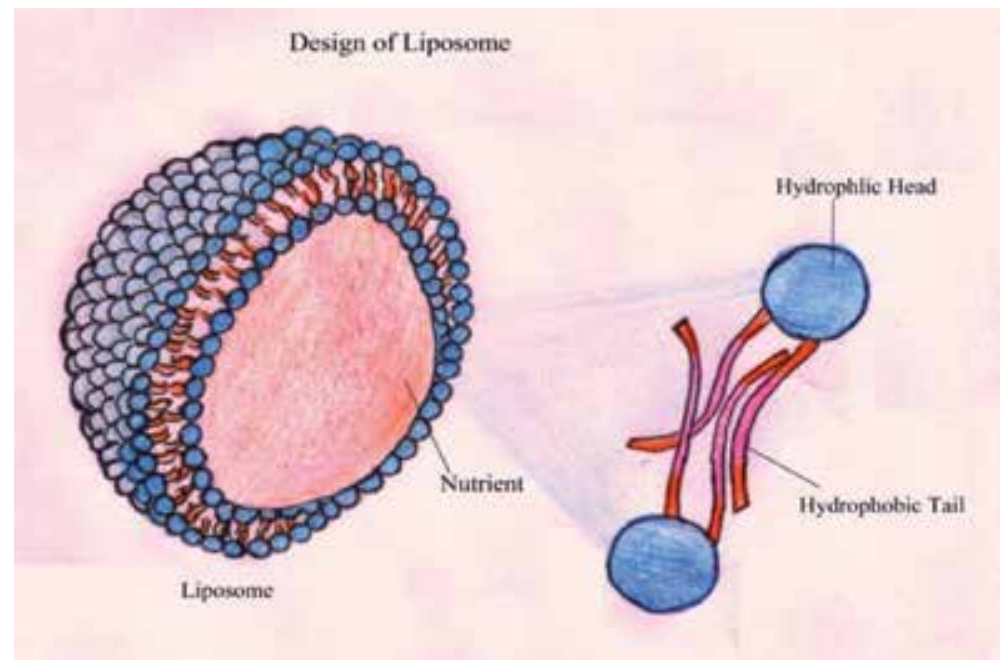

Figure 1.

Liposome (source: http://healthproductdistributor.com) [22].

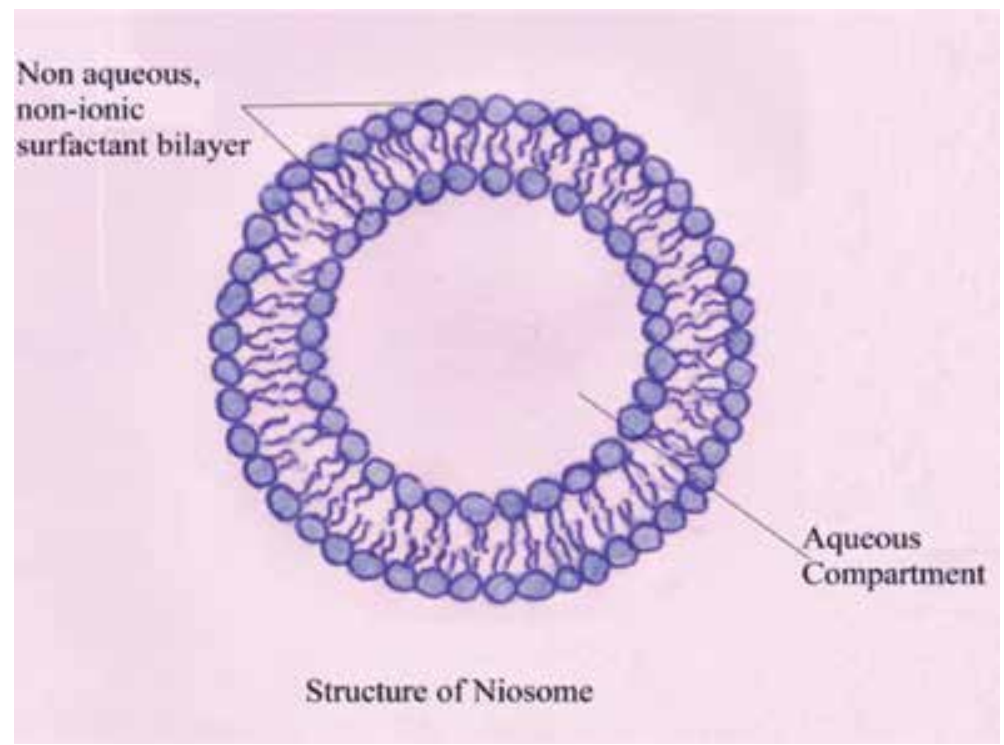

Figure 2.

Structure of niosome [24].

\subsection{Niosomes}

Niosomes are non-ionic surfactant-based liposomes. Niosomes can be obtained by hydration of liposomes. These are microscopic lamellar structures formed upon combining non-ionic surfactant of the alkyl or dialkyl polyglycerol ether class with cholesterol [15]. The stability of niosomes are affected by type of surfactant, nature of encapsulated drug, storage temperature, detergents, use of membrane spanning lipids, the interfacial polymerization of surfactant monomers in situ, inclusion of charged molecule. Niosomes have great penetrating capability [23] (Figure 2).

The sizes of Niosomes are microscopic and lie in nanometric scale. The particle size ranges from 10 to $100 \mathrm{~nm}$ [24]. 


\subsection{Discomes}

Discomes (disc shaped vesicles) are large $(16-20 \mu \mathrm{m})$ sized vesicles structures capable of entrapping water soluble solutes, formed by solubilization of niosomes with a nonionic surfactant. They act as drug reservoirs in the field of drug delivery [16].

\subsection{Transferesome}

Transferosomes are complex vesicular aggregate optimized to attain extremely flexible and self-regulating membrane; this makes the vesicles very deformable. Transferosome have the property to cross microporous barrier very efficiently, even when available passage are much smaller than the average aggregate size. Transferosome consists of natural amphipathic compound suspended in waterbased solution, something containing biocompatible surfactant. Transferosome also have lipid bilayer that surrounds an aqueous core and contain at least one component that soften the membrane and make skin more flexible. This allows an easy and rapid change in shape of transferosome [17].

\subsection{Ethosomes}

Ethosomes are lipid vesicles containing high amount of ethanol. They have the efficiency to penetrate the skin and enhance compound delivery both to deep skin strata and systemically. Ethanol fluidizes both lipid bilayers of the stratum corneum and intercellular lipid. The soft malleable vesicles then penetrate the disorganized lipid bilayer. This system consists of phospholipid, ethanol and water [18].

\subsection{Resealed erythrocytes}

The fluid portion of blood contains a large number of organic and inorganic substances in solution, which may diffusible (electrolyte, anabolic and catabolic substance formed during metabolism) and non-diffusible (proteins). The cellular portion of blood consists of erythrocytes (red blood cells), leukocytes (white blood cells) and thrombocytes (platelets). Erythrocytes have a solid content of about $35 \%$ (rest of being water) most of which is hemoglobin, which is remain tightly bound to the stroma of cell membrane [14]. Resealed erythrocytes are drug-loaded carrier erythrocytes, prepared simply by collecting blood samples from the organism of interest, separating erythrocytes from plasma, entrapping drug in the erythrocytes, and resealing the resultant cellular carriers. Hence, these carriers are called resealed erythrocytes [14].

\subsection{Aquasomes}

Aquasomes are the nano-structured biopharmaceutical vesicles based upon fundamental principle of self-assembling. It contains the particle core composed of nano-crystalline calcium phosphate or ceramic diamond, and is covered by a polyhydroxyl oligomeric film. Aquasomes are also called as “bodies of water". The drug candidates/molecule delivered by Aquasomes represent better biological activity even in case of conformationally sensitive ones. Aquasome having properties like protection and preservation of fragile biological molecules, conformational integrity, and surface exposure made it as a successful carrier system for bioactive molecules like peptide, protein, hormones, antigens and genes to specific sites [21]. 


\subsection{Phytosomes}

Phytosomes is the combination of two words, the term "PHYTO" means plant while "SOME" means cell-like [25]. The formulation is developed by encapsulating the plant material or plant extract within the spherical cell like structure, which is an advanced nano-sphere or cell forms of herbal products that are better absorbed. Phytosomes produces better pharmacokinetic and pharmacodynamic profile of drug than conventional herbal formulations. It's a novel emerging technique that is applied to phytopharmaceuticals for the enhancement of bioavailability of natural plant extract for medicinal applications. Phytomedicines, complex chemical mixture prepared from plants, have been used in India and worldwide from the very beginning of human civilization and continue to have widespread popular use [26].

Phytosome is a patented process developed by Indena, a leading supplier of nutraceutical ingredients, to incorporate phospholipids into standardized extract and so vastly improve their absorption and utilization. Phytosome is one kind of herbal extracts delivery system that deliver natural values at target site in such manner. Certain of the water-soluble phytomolecules (mainly flavonoids and other polyphenols) can be converted into lipid-friendly complexes, by reacting herbal extract owing to their enhanced capacity to cross the lipid-rich biomembranes and finally reach the blood. They have improved pharmacokinetic and pharmacological parameters which are advantageous in the treatment of acute disease as well as in pharmaceutical and cosmetic compositions [19].

Phytosomes are better able making transition from a hydrophilic environment into the lipid-friendly environment of the enterocyte cell membrane and from there into the cell, finally reaching the blood. The lipid- phase substances employed to make flavonoids lipid- compatible are phospholipids from soy, mainly phosphatidylcholine (PC). PC, the principal molecular building block of cell membranes, is miscible both in water and in oil/lipid environments and is well absorbed when taken by mouth. Precise chemical analysis indicates that a Phytosome is usually a hydrophilic herbal extract linked with at least one PC molecule. A bond is formed between the two molecules, creating a hybrid molecule. This highly lipid-miscible hybrid bond is better suited to merge into the lipid phase of the enterocyte outer cell membrane [19].

\section{Difference between phytosome and liposome}

The fundamental difference between liposomes and Phytosomes is that in liposomes the active principle is dissolved in the medium contained in the cavity or in the layers of the membrane, whereas in the Phytosome it is an integral part of the membrane, being the molecules anchored through chemical bonds to the polar head of the phospholipid [20].

There may be hundreds or even thousands of phosphatidylcholine molecules surrounding the water soluble compound in Liposome. In contrast, the Phytosome contain Phosphatidylcholine and the individual plant components actually form a 1:1 or a 2:1 complex depending on the substance. This difference show that Phytosomes being much better absorbed than liposomes. Phytosomes are superior to liposomes in skin care products [27, 28] (Figure 3).

Phytosome (lower segment) liposome (upper segment). 


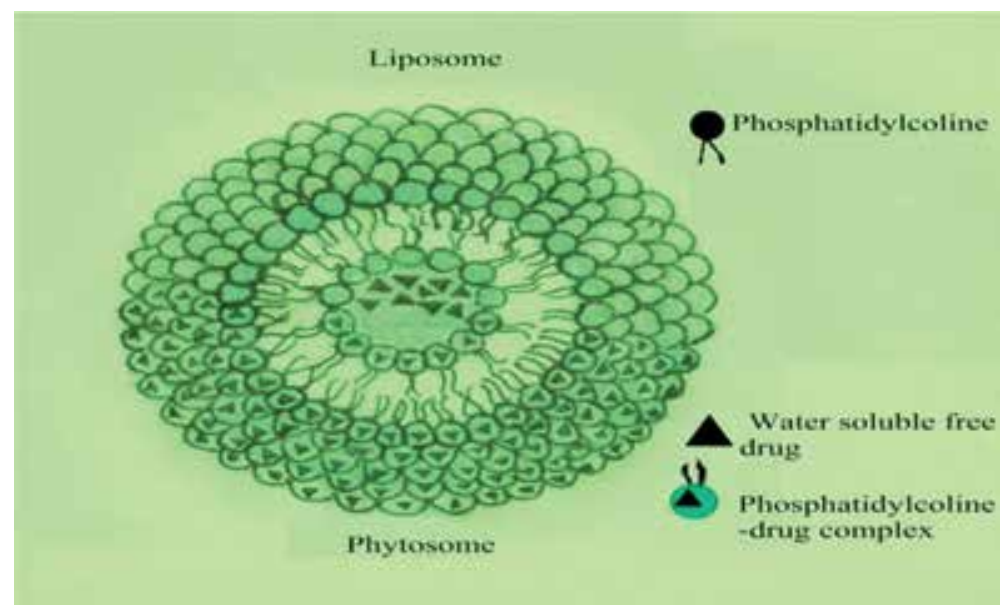

Figure 3.

Difference between phytosome and liposome [29].

\section{Merits of phytosomes over conventional dosage forms}

- The bioavailability of botanical extracts enhanced dramatically due to their complexation with phospholipids by which absorption of the constituent improved in the intestinal tract [27-30].

- Phytosme enhances the permeation of non- lipophilic botanical extract to allow better absorption from the intestinal lumen, which not possible by other carriers.

- The formulation aspect of phytosome is safe and all active components have been approved for pharmaceutical and cosmetics use.

- Due to high Bioavailability, Phytosome is being used to deliver liver protecting Flavonoids.

- This technology offers cost effective delivery of Phytoconstituents to various targeted site for pharmacological action. Phytosomes produce synergistic benefits when used as functional cosmetics, to protect the skin against exogenous or endogenous hazards in normal as well as stressful environment.

- They can be also used for enhancing permeation of drug, through skin in transdermal drug delivery system.

- They can be widely used in cosmetics due to their improved skin penetration and have a high lipid profile.

- Phosphatidylcholine, an essential part of the cell membrane and active components in phytome, act as a carrier and nourish the skin. There is no problem with drug entrapment during formulation development. The drug entrapment efficiency with phytsome is high and more over predetermined; because the drug itself forms vesicles after conjugation with lipid.

- They offer a better stability profile because chemical bonds are formed between the phosphatidylcholine molecules and phytoconstituents. 
The phytosomal system is passive, non-invasive and can is suitable for immediate commercialization.

- The dose requirement is reduced due to improved absorption of the main constituent. They can also be given in smaller quantities to achieve the desired therapeutic values.

- Low risk profile: This technology has no risk since the toxicological profiles of the phytosomal components are well documented in the scientific literature. Highly attractive market profile for products with proprietary technology. It is easy to formulate because no complicated technical investment required for the production of phytosomes.

\section{Pharmaceutical scope of phytosome}

- It enhances the absorption of lipid insoluble polar phytoconstituents through oral as well as topical route showing better bioavailability, so it has wide therapeutic benefits [28, 30].

- Appreciable drug entrapment.

- Phytosomes help to improve the absorption of active constituents and reduces the dose requirements.

- Phosphatidylcholine used in preparation of Phytosomes, besides acting as a carrier also acts as a hepatoprotective agent. Hence produce the synergistic effect to the liver targeted drug.

- Chemical bonds are formed between phosphatidylcholine molecule and phytoconstituent, this results better stability in Phytosome.

- Application of phytoconstituents in form of phytosome improves their percutaneous absorption and act as basic carrier for transdemal drug delivery.

\section{Phospholipids: creating the structural framework for novel drug delivery systems}

These are complex substance having chemical, biochemical and nutritional characteristics by which they can distinguish as placed in a unique nutritional category. They are complex lipid molecules important for life and are found in all human and the other known structure to make cell membranes. The biochemical importance of phospholipids is reflected in their extraordinary clinical benefits as dietary supplements. The phospholipids are readily compatible with the entire range of vitamins, minerals, metabolites and herbal preparations. The dietary phospholipids and omega-3 fatty acid works in functional synergy in cell membranes. Phosphatidylcholine is a bi-functional compound miscible both in water and in oil environments, and is well absorbed when taken by mouth. Phosphatidylcholine is not merely a passive "carrier" for the bioactive compounds, but is itself a bioactive nutrient with documented clinical efficacy for liver disease (including alcoholic hepatitis). Phosphatidylcholine is present in egg yolk, brain tissue and a wide 


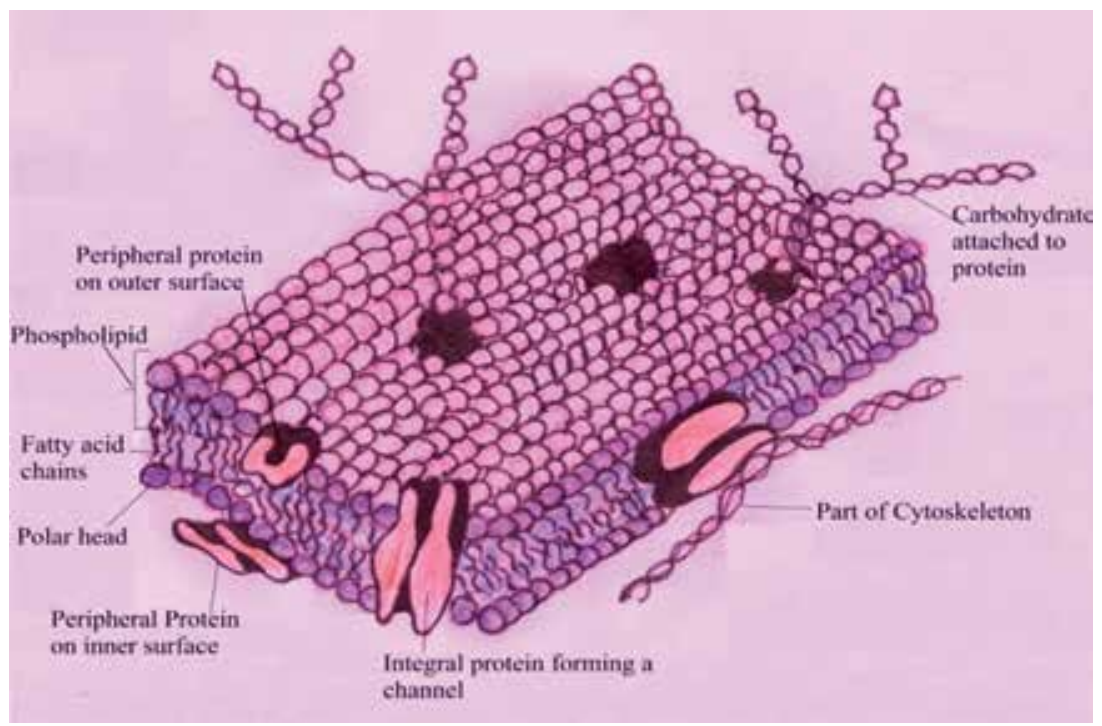

Figure 4.

Cell membranes are largely lipid phase. A double molecular layer consisting of $P C$ and other phospholipids provides a continuous matrix into which the proteins insert [31].

variety of animal fat and plant oils. It is routinely present in the bile fluid, to help emulsify food ingredient for absorption [31] (Figure 4).

A number of drug delivery system is based entirely on Phosphatidylcholine such as liposomes, ethosomes, phytosomes, transferosomes, and nanocochhelates. The hydrophilic and hydrophobic domain/segment within the molecular geometry of amphiphilic lipids orient and self-organize in ordered supramolecular structure when confronted with solvents. Some commonly used synthetic phospholipids are dioleoyl-phosphatidyl-choline (DOPC), dioleoyl-phosphatidyl-ethanolamine (DOPE), distearoyl-phosphatidyl-choline (DSPC), and distearoyl-phosphatidylethanolamine (DSPE) [31].

\section{Conjugation of NDDS with transdermal route}

Transdermal drug delivery systems (TDDS) are topically administered medications in the form of patches, which can deliver certain medication to systemic circulation in a more convenient and effective way than is possible with conventional dosage form. The potential of skin as a path of drug administration has been amply demonstrated by the acceptability of marketed therapeutic systems [32]. Administration of systemic drugs using a transdermal patch represents a noninvasive route, with improved patient compliance. This route of administration prevents passage through the gastrointestinal tract and maintains constant plasma levels for prolonged periods of time [33]. A transdermal patch is a medicated adhesive patch placed on the skin to deliver a time-released dose of medication through the skin for treating topical or systemic illness. Since early 1990, this dosage form of transdermal therapeutic system has been available in the pharmaceutical market. Such a system offers a variety of significant clinical benefits over others, such as tablet and injection [32,33]. Transdermal route is a potential mode of delivery of lipophilic drugs in the systemic circulation. Some factors are responsible for transdermal drug delivery such as area of application, amount applied, release kinetics and prolongation of application time [34]. 


\section{Feasibility of phytosome as novel vehicle for transdermal drug delivery}

Lipophilic drugs could be easily transfused by transdemal route which enhance the bioavalibility of phytoconstitutent. Drug would be delivered into systemic circulation by transdermal route transdermal route. Drug has to pass three different skin layers before reaching to systemic circulation. The outermost layer, Stratum corneum provides hindrances to such type of drugs delivery due to its lipophilicity. The Phytosomal form of phytoconstituents can pass this barrier easily and helps to deliver the phytoconstituents in systemic circulation. The research studies from two decades show the phytosome technology has enough potency to be used as novel carrier of Phytoconstituents. Phytosomal carrier has enough potency to be used transdermally for increasing bioavailability of Phytoconstituents [34, 35].

\section{Conclusion}

Ever since the advent of human civilization, man is dependent on nature to fulfill all his need. Traditional system of healing human aliment was also derived from natural resources. In an era of development, devised various means of treatment primarily based on synthetic chemicals and popularly known as allopathic system of treatment. The inherent side effects of this system led to the shift in focus from contemporary to either conventional system of medication or to evolve the Novel drug delivery system. Novel drug delivery vehicles that can be used for the poorly soluble, $\mathrm{pH}$ sensitive and less potent drug to make them safe and target oriented. These novel drug delivery vehicles can also be utilized to deliver herbal formulations to its targeted site with lesser side effect. This phenomenon can be further augmented by conjugation of transdermal drug delivery system with novel drug delivery vehicle containing nanobiomedicine as herbal constituents. Phytosomes have shown the potential to be good carrier of herbal constituents for novel drug delivery systems. The combination of Transdermal route and Phytosomal drug delivery opens up an arena for better treatment for the patient suffering from skin diseases, Burn, allergies and bone fracture.

\section{Acknowledgements}

Authors want to acknowledge the Department of Pharmaceutical Sciences, Kumaun University campus bhimtal, for providing support for library and literature search.

\section{Conflict of interest}

There is no conflict of interest. 


\section{Author details}

Mahendra Rana, Aadesh Kumar* and Amita J. Rana

Department of Pharmaceutical Sciences, Kumaun University Campus, Bhimtal, Uttarakhand, India

*Address all correspondence to: dhariwalaadesh13@gmail.com

\section{IntechOpen}

(c) 2020 The Author(s). Licensee IntechOpen. This chapter is distributed under the terms of the Creative Commons Attribution License (http://creativecommons.org/licenses/ by/3.0), which permits unrestricted use, distribution, and reproduction in any medium, provided the original work is properly cited. $(\mathrm{cc}) \mathrm{BY}$ 


\section{References}

[1] Inamdar NS, Edalat VB, Kotwal VB, Pawar SS. Herbal drugs in milieu of modern drugs. International Journal of Green Pharmacy. 2008;2:2-8

[2] Kalia AN. Text Book of Industrial Pharmacognosy. New Delhi: Oscar Publication; 2005

[3] Calixto JB. Efficacy, safety, quality control, marketing and regulatory guidelines for herbal medicines (phytotherapeutic agents). Brazilian Journal of Medical and Biological Research. 2000;33:179-189

[4] Christie A. Herbs for health, but how safe are they. The Bulletin of the World Health Organization. 2001;79:691-692

[5] Herbal Medicine Market Research Report- Forecast to 2023. Available from: http://www. markertresearchfuture.com/reports/ herbal-medicine-market-3250

[6] Mukherjee PK, Wahile A. Integrated approaches towards drug development from ayurveda and other Indian system of medicine. Journal of Ethnopharmacology. 2006;103:25-35

[7] Ajazuddin SS. Application of novel drug delivery system for herbal formulations. Fitoterapia. 2010;81:680689. DOI: 10.1016/j.fitote.2010.05.001

[8] Kidd P, Head K. A review of the bioavailability and clinical efficacy of milk thistle phytosome: A silybinphos phatidylcholine complex. Alternative Medicine Review. 2005;10(3):193-203

[9] Medina OP, Zhu Y, Kairemo K. Current Pharmaceutical Design. 2004;10:2981-2989

[10] Vyas SP, Khar RK. Targeted and Controlled Drug Delivery. 1st ed. CBS Publisher; 2013. pp. 3-10
[11] Nanoparticulate Drug Delivery. Perspectives on the Transition from Laboratory to Market. Woodhead Publishing Series in Biomedicine; 2012. pp. 29-85. DOI: $10.1533 / 9781908818195.29$

[12] Barragan Montero V, Winum J, Moles J, Juan E, Clavel C, Montero J. The European Journal of Medicinal Chemistry. 2005;40:1022-1029

[13] Chonn A, Cullis PR. Recent advances in liposomal drug delivery system. Current Opinion in Biotechnology. 1995;6:698-708

[14] Balasubramanian J, Narayanan N, Kumar V. Resealed erythrocytes: A novel drug carrier in drug delivery. Drug Discovery. 2012;2(6):30-32

[15] Malhotra M, Jain NK. Niosomes as drug carriers. Indian Drugs. 1994;31:81-86

[16] Kumarn GP, Pogaku R. Nonionic surfactant vesicular systems for effective drug delivery-An overview. Acta Pharmaceutica Sinica B. 2011;1(4):208219. DOI: 10.1016/j.apsb.2011.09.002

[17] Vyas SP. Theory and Practice in Novel Drug Delivery System. 1st ed. CBS Publisher; 2011. pp. 257-260

[18] Vyas SP. Theory and Practice in Novel Drug Delivery System. 1st ed. Vol. 2011. CBS Publisher. pp. 245-250

[19] Rathore P, Swami G. Planterosome: A potential phyto-phospholipid carrier for the bioavailability enhancement of herbal extracts. International Journal of Pharmaceutical Sciences and Research. 2012;3(3):737-755

[20] Pandey S, Patel K. Phytosomes:

Technical revolution in phytomedicine. International Journal of Pharma Tech Research. 2010;2(1):627-631 
[21] Sutariya V, Patel P. Aquasomes: A novel carrier for drug delivery. IJPSR. 2012;3(3):688-694. DOI: 10.13040/ ijpsr.0975-8232.3(3).688-94

[22] http://healthproductdistributor.com

[23] Udupa N. Niosomes as drug carriers. In: Jain NK, editor. Controlled and Novel Drug Delivery. 1st ed. New Delhi: CBS Publishers and Distributors; 2002

[24] Baillie AJ, Florence AT, Hume LR, Muirhead GT, Rogerson A. The preparation and properties of niosomes non-ionic surfactant vesicles. The Journal of Pharmacy and Pharmacology. 1985;37:863-868

[25] Jain N, Gupta BP, Thakur N. Phytosome: A novel drug delivery system for herbal medicine. International Journal of Pharmaceutical Science and Drug Research. 2010;2(4):224-228

[26] Sindhumol PG, Thomas M, Mohanachandran PS. Phytosomes: A novel dosage form for enhancement of bioavailability of botanicals and nutraceuticals. International Journal of Pharmacy and Pharmaceutical Science. 2010;2(4):10-14

[27] Sharma A, Sharma US. Review liposome in drug delivery: Progress and limitations. International Journal of Pharmaceutics. 1997;154:123-140

[28] Kidd PM. Bioavailability and activity of phytosome complexes from botanical polyphenols: The silymarin, curcumin, green tea, and Grape Seed Extracts. Alternative Medicine Review. 2005;14(3):226-246

[29] Bhattacharya S. Phytosomes: Emerging strategy in delivery of herbal drugs and nutraceuticals. Pharmacy Times. 2009;41(3):9-12

[30] Awasthi R, Kulkarni G, Pawar V. Phytosome: An approach to increase the bioavailability of plant extracts. International Journal of Pharmacy and Pharmaceutical Science. 2011;3(2):1-3

[31] Bombardelli E, Spelta M. Phospholipid-polyphenolcomplexes: A new concept in skin care ingredients. Cosmetics and Toiletries. 1991;106:69-76

[32] Modamio P, Lastra CF, Marino EL. A comparative in-vitro study of percutaneous penetration of betablockers in human skin. International Journal of Pharmaceutics.

2000;194:249-259

[33] Ke GM, Wang L, Xue HY, Lu WL, Zhang $X$, Zhang Q, et al. In-vitro and in- vivo characterization of a newly developed clonidine transdermal patch for treatment of attention deficit hyperactivity disorder in children. Biological and Pharmaceutical Bulletin. 2005;28:305-310

[34] Nicoli S, Colombo P, Santi P. Release and permeation kinetics of caffeine from bioadhesive transdermal films. The AAPS Journal. 2005;7:E218-E223

[35] Tymes NW, Shah VP, Skelly JP. In-vitro profile of estradiol transdermal therapeutic systems. Journal of Pharmaceutical Sciences. 1990;79:601-602 


\title{
Polymer Properties: Functionalization and Surface Modified Nanoparticles
}

\author{
Chander Amgoth, Chiuyen Phan, Murali Banavoth, \\ Santosh Rompivalasa and Guping Tang
}

\begin{abstract}
Herein, the various polymer properties and the underlying mechanism for the functionalization and surface modification of polymer nanoparticles have been discussed. There are numerous polymer particles designed and developed for various applications. The synthesis and characterization of different types of polymers followed by the engineering of nanoparticles and capsules depend on various factors. There are too many polymerization methods approached for the development of nanoparticles with desired surface properties. The ring-opening polymerization (ROP), emulsion polymerization (EP), atom transfer radical polymerization (ATRP), and free radical micro initiation are the significant approaches for the polymerization reactions. The polymer nanoparticle functionalization and modification of their surfaces based on requirements is an essential task. The solvent concentration, $\mathrm{pH}$, temperature, and sonication have played a vital role to tune the morphology of polymer nanoparticles and capsules. Different characterizations such as FTIR, NMR $\left({ }^{1} \mathrm{H}\right.$ and $\left.{ }^{13} \mathrm{C}\right)$, HRMS, and MALDI-TOF are used for preliminary structural and confirmations. Further, SEM, FE-SEM, TEM, AFM, BET, XRD, Raman, EDAX, TGA-DSC, DLS, and zeta potential were used for morphological and thermal properties.
\end{abstract}

Keywords: polymers, properties, functionalization, surface modification, nanoparticles

\section{Introduction}

Across the globe, there is a great interest in the field of polymers and polymerbased nanoparticles followed by their nanotechnology-based applications. However, polymeric nanoparticles (PNPs) are under a wide range of utilization in various fields. Especially researchers and scientists are trying to design various nanoparticles (NPs) and capsules with surface modifiable character features [1]. The polymer nanoparticles do obey and endorse the aforementioned sentence with existing literature which shows numerous nanoparticles with modifiable size, shape, and their surface properties. For example, quantum dots (QDs) carbon nanotubes (CNTs), cellulose NPs and fibers [2] iron oxide nanoparticles (FeONPs), gold nanoparticles (AuNPs), silver nanoparticles (AgNPs), cobalt nanoparticles (CNPs), zinc oxide nanoparticles (ZnONPs), SnO2 nanoparticles, protein-based nanoparticles, amino acid-based nanoparticles, silica ( $\mathrm{SiO} 2)$ nanoparticles, 
graphene nanosheets, titanium-based nanoparticles, core-shell nanoparticles, hollow core-shell (hCS) nanoparticles, hollow polymer-polymer nanoparticles (HPPNPs), polymer-metal nanoparticles (PMNPs), polymer-metal blends (PBs), polymer-silica nanocomposites (PSiNCs), polymeric micelles (PMs), solid-lipid nanocapsules (SLPNCs), liposomal nanoparticles, albumin-based nanoparticles, chitosan-based nanoparticles, platinum-based nanoparticles (PtNPs), ceramic nanoparticles, carbohydrate nanoparticles, etc. are reported and corroborated in the literature [2-5]. Furthermore, a significant interest in the synthesis and characterization of polymers followed by the design and development of polymeric nanoparticles (PNPs) with surface tunable properties has become an essential due to the various applications. For example, the amino acid-based polymer is much more suitable for the preparation of nanoparticles because of its biosafety and nontoxic and biodegradable nature. Similarly, thermosensitive polymer, for example, poly-Nisopropyl acrylamide (PNIPAM), shows lower critical solution temperature (LCST) at $\sim 32^{\circ} \mathrm{C}$, and it is much more useful to prepare the nanoparticles or capsules with thermally tunable morphology and other futuristic characteristic properties [6]. However, the main advantage to polymer scientists is they can easily synthesize the polymer to engineer the nanoparticles followed by the significant functionalization and surface modifications by changing the solvents, concentration, and stirring methods or by changing the functional groups which are present in the synthesized polymer complex. Similarly, the ionic (cationic and anionic) polymers referred to as polaxomers can help to modify the surface of the particles. Polymeric nanoparticles are under a wide range of utilization in the field of biomedical, nanobiotechnology, nanobiopharma, and other pharmaceutical domains. For example, they are very much used in the field of drug delivery systems (DDSs), gene delivery systems (GDSs), protein delivery systems (PDSs), cell delivery systems (CDSs), and tissue engineering followed by plastic surgery, etc. $[7,8]$. However, polymers have numerous industrial applications as well, such as cell phone manufacturing companies, soft drink and water bottle industries, electronics and electrical engineering departments, automobile industries, and many other industrial usages based on the requirements. Interestingly, polymeric nanoparticles with porosity have multidirectional usages such as drug or nanomedicine loading and its target specific release. The nanoparticles with $\sim 200 \mathrm{~nm}$ (in diameter) are the ideal size for the biomedical applications. The pore size with $\sim 100 \mathrm{~nm}$ in the polymer nanoparticles or capsules is much more suitable for drug delivery and cancer therapeutics. According to International Union of Pure and Applied Chemistry (IUPAC) definition, the microparticle size should be $<2 \mathrm{~nm}$, (in diameter) and meso-particles or mesoporous particle and capsule size ca. 2-50 nm, and nanoparticles (particle size 50-200 nm) have been referred for various applications [6-8]. The effective and potential working capacity of nanoparticles has been defined by their size, shape, morphology, porosity, and other thermal properties. However, the qualitative quantification of use of polymer-based nanoparticles and their potential applications completely depends on the characteristic features like surface modification, size, and shape tenability. For example, many properties like color and atomic orientation are differing from the sizes of bulk to nanoscale $[9,10]$.

\section{Classification of polymers}

The word polymer derived from Greek has been split into two meaningful words: (1) poly means many, and (2) meros or mers means units or parts. More specifically monomer is the smallest repeating unit for the development of polymer. Simply polymers are made up of monomers. The word monomer is also derived 
from the Greek. Mono means one or single and mer means part/s. As reported in Table 1, there are varieties of polymers classified based on their biodegradability, biocompatibility, thermal stability, $\mathrm{pH}$-responsive nature, environmental responsive nature, etc. Based on occurrence these are again categorized into three types: (1) natural polymers, for example, starch, chitin, cellulose, etc., (2) synthetic polymers, for example, polystyrene (PS), polyurethane (PU), polyvinyl chloride (PVC), and (3) semisynthetic polymers, for example, cellulose nitrate, guncotton, cellulose acetate, etc. Absorbable synthetic polymers include polyvinyl alcohol (PVA), polylactic acid (PLA), polycaprolactone (PCL), and polyglycolic acid (PGA), and these are homopolymers. The heteropolymers include polytrimethylene carbonate (PTMC), polyetherimide (PEI), polydioxanone (PDO), polylactide-co-glycolide (PLGA), and the combination of block copolymers like and poly-glycolide-co-trimethylene carbonate (PGTMC) which are best examples for heterogeneous polymers. Apart from these polymers, there are $\mathrm{pH}$-sensitive polymers like poly-L-lysine which is positively charged at lower $\mathrm{pH}$ conditions $[22,23]$. Liposome and poly-histidine are the systems with $\mathrm{pH}$ sensitivity, and this can be easily interacted with negatively charged particles or membranes to promote fusogenic properties. Furthermore, some hydrogel-based nanoparticles show unique characteristics toward various biomedical as well as industrial applications. Liposomal and hydrogel based systems has tendency towards $\mathrm{pH}$-sensitivity and it is a characteristic property to tune-up their morphology for many therapeutic applications. For example, PLGA, MeO-PEG- $\mathrm{NH}_{2}$, and PAMAM-based nanoparticles show $\mathrm{pH}$-dependent destabilization and thus are used for cytoplasmic experiments because of its ease of permeability through the membrane to deliver nanomedicines and nanoparticles $[9,10,22,23]$. The NIPAM/pNIPAM (N-isopropyl acrylamide/ (poly-N-isopropyl acrylamide)) is the best example for the thermal sensitive polymer which shows lower critical solution temperature (LCST) or cloud point at $\sim 32^{\circ} \mathrm{C}$ Table 1 . Because of the thermal behavior, it is termed as magic or smart polymer and used for various medical and industrial usages. However, these polymers are mainly prepared based on two polymerizations reactions such as (1) addition polymerization and (2) condensation polymerization methods [24, 25].

\begin{tabular}{|c|c|c|c|c|c|}
\hline $\begin{array}{l}\text { S. } \\
\text { no. }\end{array}$ & Name of the polymer & $\begin{array}{l}\text { Monomer } \\
\text { formula }\end{array}$ & $\begin{array}{l}\text { Polymer } \\
\text { formula }\end{array}$ & $\begin{array}{l}\text { Polymer } \\
\text { structure }\end{array}$ & Ref. \\
\hline 1 & PVC (polyvinyl chloride) & $\mathrm{C}_{2} \mathrm{H}_{3} \mathrm{Cl}$ & $\left(\mathrm{C}_{2} \mathrm{H}_{3} \mathrm{Cl}\right)_{\mathrm{n}}$ & Figure 1a & {$[11,12]$} \\
\hline 2 & PVA (polyvinyl alcohol) & $\mathrm{C}_{2} \mathrm{H}_{4} \mathrm{O}$ & $\left(\mathrm{C}_{2} \mathrm{H}_{4} \mathrm{O}\right)_{\mathrm{n}}$ & Figure 1b & {$[12,13]$} \\
\hline 3 & PE (polyethylene) & $\mathrm{C}_{2} \mathrm{H}_{4}$ & $\left(\mathrm{C} 2 \mathrm{H}_{4}\right)_{\mathrm{n}}$ & Figure 1c & {$[12,14]$} \\
\hline 4 & PS (polystyrene) & $\mathrm{C}_{8} \mathrm{H}_{8}$ & $\left(\mathrm{C}_{8} \mathrm{H}_{8}\right)_{\mathrm{n}}$ & Figure 1d & {$[14,15]$} \\
\hline 5 & PP (polypropylene) & $\mathrm{C}_{3} \mathrm{H}_{6}$ & $\left(\mathrm{C}_{3} \mathrm{H}_{6}\right)_{\mathrm{n}}$ & Figure 1e & {$[15,16]$} \\
\hline 6 & $\begin{array}{l}\text { NIPAM } \\
\text { (isopropylacrylamide) }\end{array}$ & $\mathrm{C}_{6} \mathrm{H}_{11} \mathrm{NO}$ & $\left(\mathrm{C}_{6} \mathrm{H}_{11} \mathrm{NO}\right)_{\mathrm{n}}$ & Figure 1f & {$[16,17]$} \\
\hline 7 & PTFE (Teflon) & $\mathrm{C}_{2} \mathrm{~F}_{4}$ & $\left(\mathrm{C}_{2} \mathrm{~F}_{4}\right)_{\mathrm{n}}$ & Figure 1g & {$[17,18]$} \\
\hline 8 & PCL (polycaprolactone) & $\mathrm{C}_{6} \mathrm{H}_{10} \mathrm{O}_{2}$ & $\left(\mathrm{C}_{6} \mathrm{H}_{10} \mathrm{O}_{2}\right)_{\mathrm{n}}$ & Figure $1 \mathrm{~h}$ & {$[18,19]$} \\
\hline 9 & PEI (polyetherimide) & $\mathrm{C}_{37} \mathrm{H}_{24} \mathrm{O}_{6} \mathrm{~N}_{2}$ & $\left(\mathrm{C}_{37} \mathrm{H}_{24} \mathrm{O}_{6} \mathrm{~N}_{2}\right)_{\mathrm{n}}$ & Figure 1i & {$[19,20]$} \\
\hline 10 & PSU (polysulfone) & $\mathrm{C}_{27} \mathrm{H}_{22} \mathrm{O}_{4} \mathrm{~S}$ & $\left(\mathrm{C}_{27} \mathrm{H}_{22} \mathrm{O}_{4} \mathrm{~S}\right)_{\mathrm{n}}$ & Figure $1 \mathbf{j}$ & {$[20,21]$} \\
\hline
\end{tabular}

Here, " $n$ " represents the number of monomer units involved in the synthesis of the polymers.

Table 1.

Show the different types of polymers with their monomer units and their chemical formula followed by the structures in Figure 1a-j. 


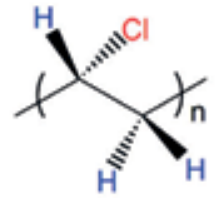

(a) PVC

(Polyvinyl chloride)

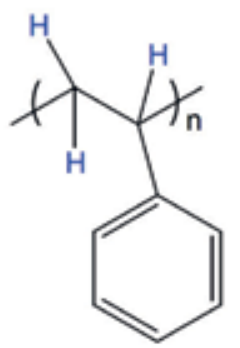

(d) PS

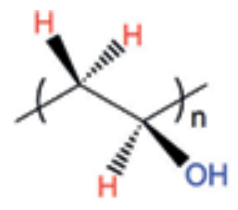

(b) PVA (Polyvinyl alcohol)

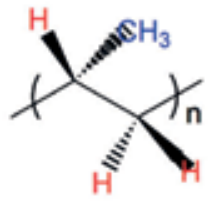

(e) PP (Polypropylene)<smiles>CCCCC(C)C</smiles>

(c) PE (Polyethylene)<smiles>CC(C)NC(=O)C(CC(C)(C)C)C(C)(C)C</smiles>

(f) P/NIPAM

(Poly-N-isopropyl acryl amide)

(Polystyrene)<smiles>CC(C)(C)C(F)(F)C(F)(F)C(F)(F)F</smiles>

(g) Teflon (Polytetrafluoroethylene)<smiles>CCC(=O)OCCCCCC(C)(C)C</smiles>

(h) PCL

(Polycaprolactone)<smiles>CCCCCC(C)(C)c1ccc(Oc2ccc3c(c2)C(=O)N(c2ccc(C(C)(C)C)cc2)C3=O)cc1</smiles>

(Polyetherimide)

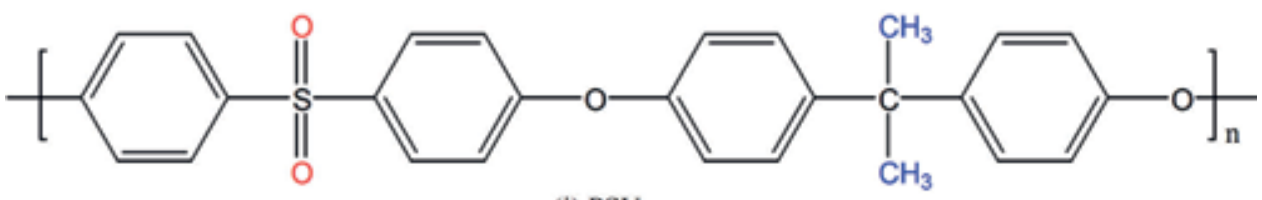

(j) PSU

(Polysulfone)

Figure 1.

Some of the polymers with their chemical structure, name, and active functional groups which are involved in functionalization and polymerization reactions.

Hence, polymers are prepared by the abovementioned two versatile polymerization methods, and there are several modified methods and approaches that can be followed for the preparation and synthesis of polymers such as ring-opening polymerization (R-O-P), emulsion polymerization, precipitation polymerization, diffusion polymerization, macroinitiator polymerization, macroinitiator polymerization, and atom transfer radical polymerization (ATRP) methods. As per the literature, first in the 1970s, lactic acid (LA)-based nanoparticles and capsules are 
used for biomedical and other pharmaceutical applications, and later it has been extended for various fields [26]. Polymers when considered for biomedical applications, it needs to refer self-assembly nature and functionality followed by the formation of spherical and sphere-shaped nanocapsules and particles particularly for drug delivery systems. Based on degradability, thermosensitivity, and $\mathrm{pH}$ responsive nature, these are again categorized into various classes and reported in Figure 2 with some examples. However, biodegradable polymers which show a tendency to degrade in biological systems such as polylactic acid (PLA), polyglycolic acid (PGA) and poly terephthalic acid (PTA), polycaprolactone (PCL), and amino acid (AA)-based polymers are potential materials to engineer nanoparticles and capsules for nanotechnology and nanobiotechnology applications. The polymers which do not degrade in the biological systems are termed as non-biodegradable polymers such as carboxymethyl, ethyl cellulose, and acetates of cellulose. The poly anhydrides like poly sebacic acid (PSA), poly adipic acid (PAA), and other environmental-responsive polymers also enlisted in the non-biodegradable polymers. Thermo-responsive (temperature-sensitive) polymers show a significant change in the size and shape of nanoparticles to tune up based on requirements [27]. The thermosensitive behavior of PNIPAM and $\mathrm{pH}$-responsive nature of L-glutamic acid gamma benzyl ester (L-GluA-5-BE), L-aspartic acid beta benzyl ester (L-AspA4-BE), polyethylene glycol (PEG), poly L-glycolic acid (PLGA), and PAMAM allow changing the morphology of nanoparticles as well surface profile of the NPs and capsules (Figure 1). Apart from these polymers, electrical-responsive polymers show piezoelectric effects which are a characteristic property to mold the nanoparticles based on requirements. The supramolecular interactions between the atoms and molecules lead to compression or elongation and stretching of bonds, and it can play a vital role to change the properties of final compounds. However, polyvinylidene fluoride (PVDF) polymer has been considered as one of the magic polymers which allow structural and morphological modifications through the appropriate synthesis and preparation methodologies [28].

The chemically responsive polymers allow internal changes through the formation of anionic and cationic and amphiphilic nature through the oxidation, reduction, and redox (both oxidation and reduction) reactions and lead to surface modification based on smooth and rough surface. As per the literature, the increase in the size of the particles depends on an increase in monomer units or polymer units. Sometimes branching and hyper-branching nature also lead to the formation of larger nanoparticles and capsules. Polymers prepared with the

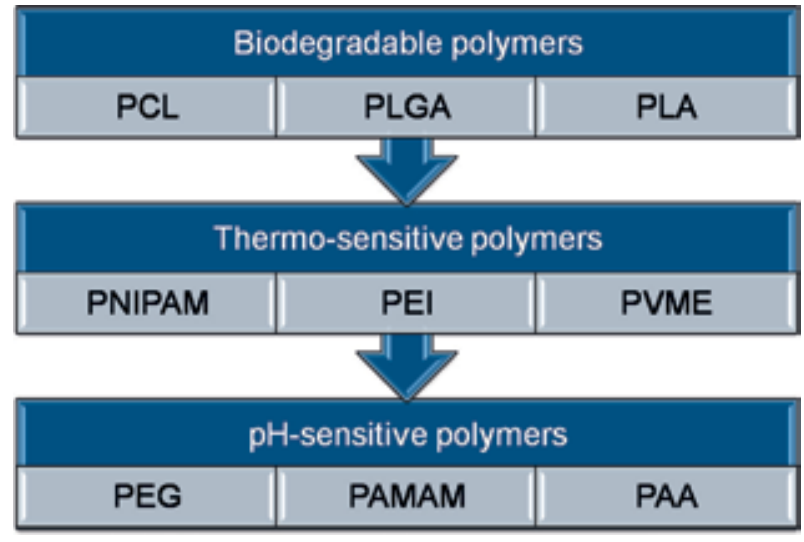

Figure 2.

Some of the polymers categorized based on their characteristic properties. 
combination of a large number of high molecular mass polymers give micronsized nanoparticles, and they can be used for bulk phenomenal approaches such as absorption, chemisorption, etc. There is a class of polymers named as block copolymers, and these are again categorized into several such as (a) random block copolymers (BCPs), (b) star block copolymers, (c) grafted block copolymers, and (d) alternating block copolymers, and these are classified based on their synthesis approaches and final structural confirmations, and these are low-density polymers (Figure 2) [20, 21, 26-29].

\section{Polymer properties}

There are some important physical and chemical properties needed to be considered before selecting polymers for synthesis followed by the design and development of nanoparticles such as (1) structural integrity, (2) low immunogenic nature, (3) biocompatibility (for biomedical applications), (4) biologically active nature, and (5) structural versatility (which can easily allow topographic changes internally as well externally). Polymers with physicochemical properties such as hydrophilic (solvent loving), hydrophobic (solvent repelling), and amphiphilic (affinity to absorb and repel the solvent) nature, surface energy, viscosity, lubricity, density, surface tension, $\mathrm{pH}$ sensitivity, smoothness, swelling nature, solubility, amorphous and crystalline nature, polymer dissolution, bioadhesive and sticky nature, polymer-solvent interaction, polymer erosion, flexibility, chain formatting nature, chemical composition, microstructural design, and other surface properties need to be examined carefully for the design of nanoparticles and capsules. By considering these properties, nanoparticle preparation methods can also be modified for the polymerization reactions (Figure 3). For example, (1) natural polymers like (a) chitin, (b) cellulose, and (c) starch can be widely used for nanoparticle development through the modified chemical synthesis methods [29, 30]. Further, (2) semisynthetic polymers like (a) cellulose nitrate, (b) rubber, (c) rayon and (d) silk and fiber-based materials, and (e) guncotton are synthesized based on their flexibility and other stresses and strain rate for various applications, and these are very much suitable to design the ribbon type of fibrils for textile industry-based usages. However, (3) synthetic polymers such as (a) polymethyl methacrylate amide (PMMA); (b) nylon, nylon-6, and nylon-6,6; and (c) polystyrene (PS) are very much useful for plastic industries to manufacture polyvinyl chloride (PVC)based pipes, tubes, cables, and other plasticwares. The polymer tetrafluoroethylene (Teflon or PTFE) with monomer chemical formula $\left(-\mathrm{C}_{2} \mathrm{~F}_{4}-\right)$ is under wide range utilization in the name of Teflon tape as a sealant for pipe linkages and insulation tape. The natural rubbers isoprene and neoprene can be used to develop various products industrially $[31,32]$.

The natural rubber, vulcanized rubber, or sulfur has been used in tire industries. Similarly, formaldehyde and benzophenol combination is used to develop combs and other plasticwares, and its trade name is Bakelite. The polymer polyethylene $(\mathrm{PE})$ is used for polyethylene covers/carriage bags and much more houseware and industrial manufacturing. The polymer $\mathrm{PE}$ is again categorized into two types based on its density for the manufacturing of plastic materials such as low-density polyethylene (LDPE) and high-density polyethylene (HDPE) based on applications [33]. The following properties of polymers are essential to develop potential nanoparticles for drug delivery systems (DDSs), gene delivery systems (GDSs), cell delivery systems (CDSs), tissue engineering (TE), and other nanobiotechnology applications such as molecular weight, self-assembly nature, density, network 


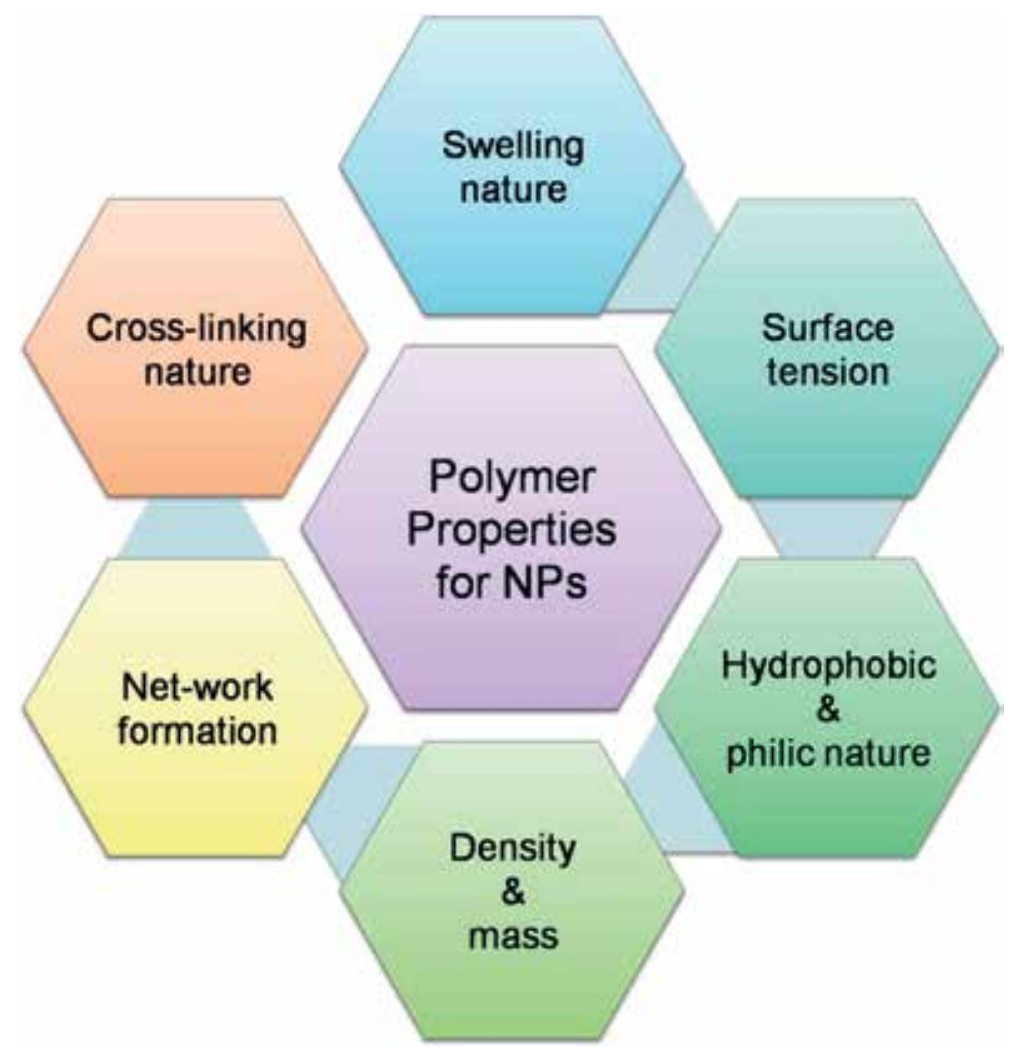

Figure 3.

Schematic shows the characteristic properties of polymers needed to be considered for the design and development of nanoparticles.

forming capacity, and interlocking nature of functional groups followed by the selfassembly. Based on applications (biomedical or industrial), polymeric nanoparticles should possess the following characteristics such as biocompatibility and biodegradability with good cell survival rate, and they should obey the properties as mentioned in earlier sections [34]. However, every nanoparticle system has their limitations for their use in medical biotechnology and nanotechnology applications. By changing reaction methods, solvent concentration, temperature conditions, $\mathrm{pH}$ levels, its surface profile and another size, shape, and morphology can be modified [35]. Design and development of surfactant-free nanoparticles without using any harmful solvents and catalyst is a novel approach for green synthesis and technology. The term green synthesis, green technology, green nanoparticles, and green reactions define the methods and approaches followed by engineer nanoparticles and materials without any side effects, toxicity, and harm to mankind applications. The nanoparticles and capsules developed based on amino acid-based block copolymers, and plant extracts are termed as green nanoparticles as they show good biocompatibility for human beings [36].

\section{Classification of nanoparticles}

Based on core synthesis materials, nanoparticles are categorized into various types such as (a) protein nanoparticles (PrNPs), (b) polymeric nanoparticles (PNPs), (c) organic nanoparticles (ONPs), (d) inorganic nanoparticles (INPs), 
(e) metal nanoparticles (MNPs), (f) metal oxide nanoparticles (MONPs), (g) amino acid-based nanoparticles (AANPs), (h) ceramic nanoparticles (CNPs), (i) hydrogel nanoparticles (HNPs), (j) solid-lipid nanoparticles (SLNPs), etc. [37-40].

However, these polymer-based nanoparticles and capsules are again classified into several categories as follows: (a) polymer-polymer nanostructures, (b) polymer-metal NPs, (c) polymer-nonmetal NPs, (d) polymer-inorganic NPs, and (e) polymer-organic (proteins, amino acids) NPs. However, polymer-polymer nanoparticles, polymer-silica NPs, amino acid-based polymers, and polymer-zinc oxide NPs have unique characteristic properties for various biomedical applications such as drug delivery systems (DDSs). Based on cytotoxicity, side effects and biocompatibility issue the quantum dots (QDs), and carbon nanotubes (CNTs) are avoided for the usage in the field of biomedical and pharmaceuticals. Aforementioned, there are various polymer-based nanoparticles which are already developed, but they all have their limitations, and for amino acid-based polymer nanoparticles and nanocapsules (NCs) like dendrimers, core-shell nanoparticles (CSNPs), the block copolymer (BCP), and mesoporous $\mathrm{SiO}_{2} \mathrm{NPs}$, it is easy to change their surface and other properties [41]. However, Figures 4 and 5 illustrate the classification of polymer and polymer nanoparticles and non-polymeric nanoparticles. The concept self-assembly has an obligatory role to rearrange and orient the active functional groups present in the monomers and polymers through the esthetic structural integrity throughout the surface of the nanoparticles and capsules. The concept self-assembly as well amorphous and crystalline nature of polymer are also one of the key factors which influence the arrangement and organization between functional groups like hydroxyl $(-\mathrm{OH})$, amine $\left(-\mathrm{NH}_{2}\right)$, amide $(-\mathrm{NH})$, keto $(-\mathrm{C}=\mathrm{O})$, ester $(\mathrm{R}-\mathrm{COO}-\mathrm{R})$, acid $(-\mathrm{COOH})$, aldehyde $(-\mathrm{CHO})$, and saturated and unsaturated hydrocarbons as discussed in earlier sections [38-43]. The formation of porous spheroid-shaped nanoparticles and capsules depends on intra- and intermolecular interactions between functional groups. The molecular imprinting of hydrophilic nanoparticles with denser hydrophobic particles can lead to the formation and creation of pores within the particles and throughout the surface of the particles and capsules [41-44].

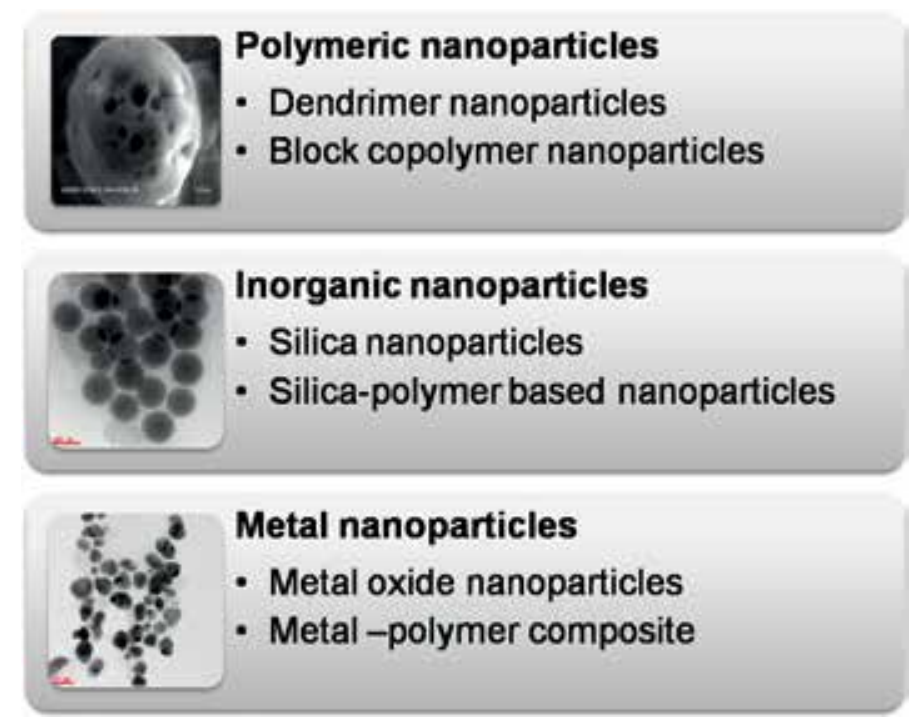

Figure 4.

Shows the classification of various nanoparticles and their morphology acquired through the different microscopic characterizations. 


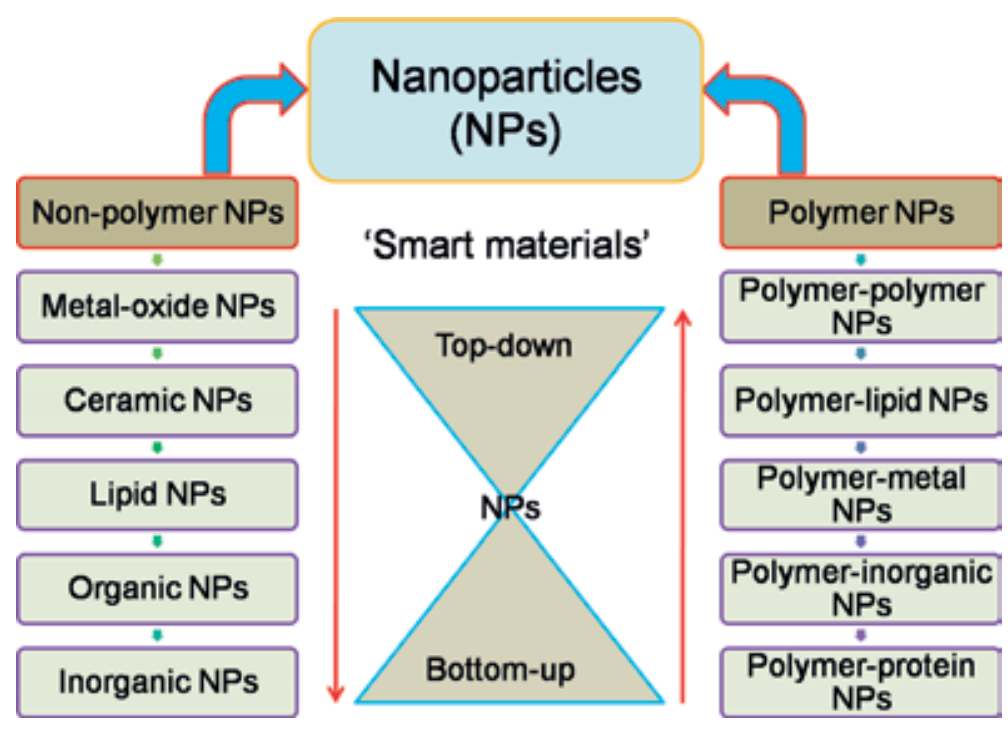

Figure 5.

Schematic illustration shows the classification of different types of nanoparticles and their synthesis approach methods.

Nanotechnologically there are two important approaches to prepare the nanoparticles such as top-down approach and bottom-up approach. However, topdown approach can be termed as breakdown approach, and it is the core method to prepare nanoparticles. For example, bulk gold cube to gold nanoparticles (AuNPs) can be achieved through the top-down approach, and simply particle segregation takes place during this approach [42-45]. Sonication, ultra-sonication, ball milling, and stirring are the best examples for the top-down approach to preparing nanoparticles. Similarly, bottom-up can be referred to as the buildup approach and in this approach nano to macro and macro to bulk materials can be achieved. This bottomup approach is like aggregation of nanoparticles which leads to the formation of bulk materials. However, monomer to polymer formation is the best example for the bottom-up approach. Sometimes based on potential applications, these nanoparticles are called smart materials or smart nanoparticles (Figure 5) [43-46].

\section{Advantages with polymer nanoparticle}

These polymer-based nanoparticles are developed in principle to protect the guest particles or molecules from degradation and its functional activity, for example, porous polymer nanoparticles or nanocapsules loaded with anticancer drugs or nanomedicines [47]. Because of the soft nature of polymer NPs and nanocapsules, it is very easy to modify the surface of the particles and their monodispersion based on its applications. The enhanced absorption and modification of the drug distribution throughout the surface of the particles or capsules lead to the target-specific or tissue- or cell- or organ-specific pharmacokinetics and dynamics in various biomedical and pharmaceutical usages. Polymer-based nanoparticles and capsules allow easy surface functionalization and modification to tune up them with desirous properties. Sometimes smooth surface of the polymer thin films can become rough due to the internalization and incorporation of metal and other inorganic nanoparticles such as FeONPs, ZnONPs, AlNPs, AgNPs, AuNPs, $\mathrm{SiO}_{2} \mathrm{NPs}$, etc. The surface properties like particle smoothness and roughness or porous nature of particles in composite can give better results, but its surface and size may not fit for 
the usage of biomedical applications [48]. In such cases the composite of polymer thin film embedded or doped with some other metal nanoparticles or organic and inorganic materials needs to be molded without changing its original activity or characteristic properties. However, the functional groups such as amide $(-\mathrm{NH})$, amine $\left(-\mathrm{NH}_{2}\right)$, acid $(-\mathrm{COOH})$, aldehyde $(-\mathrm{CHO})$, keto $(-\mathrm{C}=\mathrm{O})$, ester $(\mathrm{R}-\mathrm{COO}-\mathrm{R})$, anhydride (R-CO-O-CO-R), acid halides, hydrogen halides, salts, and solvents (organic, inorganic) can also influence the surface properties for the nanoparticles or nanocapsules. Based on the requirement, it can be chosen to modify the particles surface and size, shape, etc. The concentration of the solvent also affects the pattern of the nanoparticles and nanocapsules. Sometimes additives like precursors and surfactants can also show an adverse effect on the morphology of polymer nanoparticles [49]. The activity (bioactive nature) of polymer nanoparticles and nanocapsules can disturb the body metabolism. The active functional groups with their potential resonance capabilities can also interact with body fluids and interfere to shore-up the mutations. Apart from the solvent, there are several factors which can affect the patterning of the surface of the nanoparticles such as $\mathrm{pH}$, thermal stability, rubbery nature, solid (amorphous and crystalline) nature, environmental conditions, etc. The stability, biocompatibility, and route or synthesis methodology are also playing a vital role to tune up the surface properties of the polymeric nanoparticles and capsules. For example, (a) protein nanoparticles have stability issues during storage. If the storage conditions are not followed properly, they may get contaminated and become toxic [50]. But these protein-based NPs are very much compatible with their unique characteristic features such as nontoxic nature and nonantigenic nature. As per the existing literature, it is known that bulk protein in plasma can easily bind to a variety of drug molecules through its trajectory while carrying them to deliver target specifically. And these are very much flexible to ease scale-up manufacturing and other nanobiotechnological applications; (b) albuminbased nanoparticles are very much suitable for the morphological modifications and to tune the surface properties. These are biocompatible and biodegradable without any detrimental activities upon mankind usages. Sometimes these albumin-based nanoparticles are used for drug delivery and other biomedical applications.

However, protein albumin contains a huge number of charged amino acid residues, and it binds water-soluble drugs through charge interactions, and it leads to surface modifications and morphological changes [51].

\section{Preparation of polymer nanoparticles}

There are several approaches to prepare and synthesize the polymeric nanoparticles such as (a) desolvation, (b) emulsification, (c) esterification, (d) thermal gelation, (e) nanospray drying, (f) self-assembly, etc. Apart from these approaches, there are mainly two polymerization methods widely followed for the preparation of polymer nanoparticles such as (1) addition polymerization and (2) condensation polymerization. The preparation of polymer nanoparticles is not an easy task. The size and shape of the particle are a very important concern while preparing the polymer-based nanoparticles. As the number of monomers or polymer units increases, the size of the nanoparticle or capsule also increases. It is easy to prepare and reproduce the polymeric nanoparticles with the porous matrix for loading the drug molecules, but its size and shape cannot be formulated easily. Amino and carboxyl functional groups permit functionalization and surface modifications, but patterning them in a fruitful design is a challenging task, and it can overcome through the detailed study of their properties [52]. However, albumin is phase separated due to reduced water solubility followed by the formation of the tiny particles 
(a)

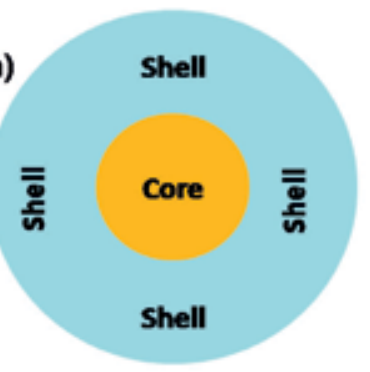

(d)

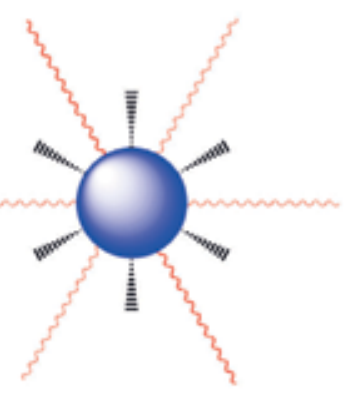

(b)
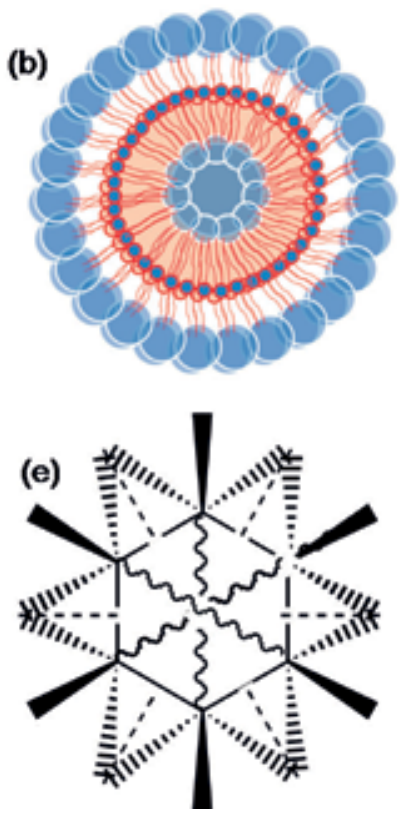

(c)
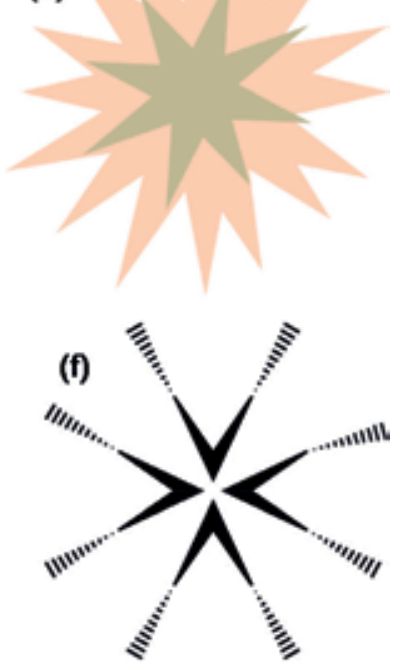

Figure 6.

Pictorial representation of different types of polymer nanoparticles. Image (a) core-shell nanoparticle, (b) liposomal nanoparticle, (c) star-shaped NPs, (d) dendritic nanoparticles, (e) coral reef-type nanoparticle, and (f) symmetric pattern of nanoparticles [46-51, 53].

such as coacervates which has a tendency to dissolve again and again with universal solvent. Albumin functional groups are cross-linked by agents like glutaraldehyde, and this cross-linking is achieved through the lysine and arginine amino groups which are present in the so-called amino acids. The stability of these cross-linked nanoparticles also affects the surface properties of the nanoparticles. Furthermore, separation methods, stirring methods, time, temperature, ultrasound, magnetic effect, sonication time, and bath temperature also show an adverse effect on the surface morphology of albumin nanoparticles.

There are significant advantages with the aforementioned approaches to prepare the polymer nanoparticles, emulsification technique requires removal of organic solvents and surfactants, thermal stabilization method is applicable to heat-stable molecules, chemical stabilization is needed to remove the toxicity of cross-linkers and uncontrolled linking, desolvation leads to the substitution for the emulsification and reproducibility with safe and suitable properties for the preparation of polymeric nanoparticles, and Figure 6a-f shows the morphology of different types of nanoparticles designed for pictorial representation $[54,55]$.

\section{Characterizations of nanoparticles}

Variety of techniques used to find out the chemical formula and structural confirmations of polymers after synthesis, for example, nuclear magnetic resonance (NMR) followed by the ${ }^{1} \mathrm{H}$ and ${ }^{13} \mathrm{C}$ for functional groups, chemical formula, and structure of the synthesized compound. Spectrophotometer fourier transform infrared (FTIR) for functional groups identification and it has been acquired through the absorption spectra followed by the analysis of peak positions, highresolution mass spectrometer (HRMS), and low-resolution mass spectrometer to measure the molecular weight of samples, and this technique give very much 
accurate mass of the synthesized compounds, matrix-assisted laser desorption ionization-time of flight (MALDI-TOF) to measure the molecular mass of heavier compounds such as polymer macromolecules, mass of genetic materials such as deoxyribonucleic acid (DNA), ribonucleic acid (RNA) and other biomolecules, carbon, hydrogen, nitrogen, sulfur (CHNS) analyzer. Once the synthesized molecules are confirmed, then it needs microscopic characterizations for the morphology of polymeric nanoparticles. Morphology of polymer nanoparticles has been characterized by using scanning electron microscope (SEM), field emission scanning electron microscope (FESEM), transmission electron microscopy (TEM), high-resolution transmission electron microscopy (HRTEM) for morphology of nanoparticles, X-ray diffraction (XRD) to know the solid state and nature of materials such as amorphous, crystalline and partially crystalline nature of synthesized nanoparticles, energy dispersive spectroscopy (EDAX) for the presence of elements and elemental composition in the final compound, Brunauer-Emmett-Teller (BET) to measure the BET surface area, porosity, pore width, pore thickness, specific surface area, Langmuir surface area through the nitrogen $\left(\mathrm{N}_{2}\right)$ gas adsorption and desorption hysteresis curve analysis, Atomic force microscope (AFM) for surface properties like smooth/hard/rough surface of the polymer and non-polymer nanoparticles followed by the pore profile such as pore depth and width in case of porous capsules or particles, apart from this characterization Optical profilometer can also use to evaluate the surface properties of nanoparticles and thin films. To examine the thermal properties and degradation behavior of nanoparticles, the following characterization techniques are used such as differential scanning calorimetry (DSC) for heat flow measurements such as endo- and exothermic nature of particles. Endothermic has been defined as the system which absorbs heat from surrounding and exothermic the system which releases heat to surroundings, thermogravimetric analysis (TGA) for weight loss and degradation temperature of various nanoparticles, Raman to measure the band gap, dynamic light scattering (DLS) for particle size analysis, zeta potential to measure the charge of the polymeric and non-polymeric nanoparticles.

\section{Preparation and functionalization of polymer nanoparticles}

However, polymer nanoparticles can be prepared through various approaches through the monomer to polymer formation trajectory which is scientifically called as polymerization reactions followed by the development of nanoparticles. Interestingly, the group transfer polymerization (GTP), ring-opening polymerization (ROP), radical polymerization (RP), polymer re-precipitation (PRP), emulsion, micro radical polymerization (MRP), macro polymer initiation reactions (MPIR), addition polymerization reactions (APR), emulsion polymerization (EP), atom transfer radical polymerization (ATRP), addition polymerizations (AP), condensation polymerization $(\mathrm{CP})$, oxidation polymerization $(\mathrm{OP})$, and reduction polymerization (RDP) followed by the redox reactions and self-assembly approach of polymer materials are used to design and develop nanoparticles from the synthesized polymers. The hydrophobic and hydrophilic nature of polymers followed by the amphiphilic character has been considered while performing the polymerization reactions to develop nanoparticles [52-56]. After synthesis of the polymer by using any of the abovementioned polymerization reactions, the final compound can be used for morphological characterizations. Sometimes solid powder form of the sample can be used directly for SEM and TEM characterizations. But for polymeric samples, it needs to be dispersed in the suitable solvent before going 
for morphological characterizations. Dispersion followed by the sonication, stirring, ultra-sonication, shaking, etc. based on particle aggregation and segregation tendency dispersion methods needs to be chosen. During the dispersion followed by the sonication, there are certain precautions needed to be taken care of such as sonication time, bath temperature, etc. Bath temperature and sonication time have to be optimized; otherwise structural disintegration or polymer particles may get collapsed or disrupted with over sonication and temperature conditions. Apart from the abovementioned methods, there are several modified chemical synthesis routes to approach to elucidate the nanoparticle design and development based on their applications. Nanoparticle formulation and functionalization depends on the synthesis methods and desired characteristic features of the polymers chosen [57]. Charged nanoparticles may have the tendency to attract or repel with other nanomaterials. In general, the size of nanoparticles increases upon blending with some other nanomaterials, and it leads to form bulk materials. For example, polystyrene (PS) nanoparticles followed by the sulfonation lead to increase the composite size. The swelling and dispersion and diffusion nature of nanoparticles lead to enhance the size of nanoparticles, and this is because of the additional functionalization. The monodispersion approach helps to get the uniform-sized nanoparticles with desired surface properties [58]. The synthesis approaches, nanoformulations, and functionalization of nanocarrier capsules and particles are very important in the drug delivery systems. And this can be achieved through the synthesis methods like ring-opening polymerization, polymer precipitation, selfassembly of polymer materials, etc. Polymers are having the capability to form the network-like structures due to its functional group entanglement and orientation to get the stability in its structure internally. The chain cross-linking and branching nature hyper-branching also lead to the functionalization of polymer nanoparticles. The synthesis of nanocarriers like nanocapsules, nanospheres, and nanoparticles with the characteristic features such as with the mesoscale porosity (pore diameter is $2-50 \mathrm{~nm}$ ), nanoporous (pore diameter $\sim 100 \mathrm{~nm}$ ), microporous (pore diameter $\sim 2 \mathrm{~nm}$ ) and cavities, voids, and core-shell concept is important to absorb or encapsulate and entrap the drug molecules [59].

\section{Morphology and surface patterning of nanoparticles}

Scientists already developed different advanced techniques to examine the meso-, nano-, and micro-, and macroscale porosity within the polymer nanoparticles, and this porous surface patterning depends on the self-assembly of chosen materials. Polymer nanoparticles' preparation is fairly easy, and they show good control over the size and shape with longer clearance time. The extensive use of polymer nanoparticles has led to study the surface properties based on its pharmaceutical and industrial applications [57-61]. For example, PVA and PLA nanoparticles show unique characteristic properties to modify their surface with greater control of size. However, double emulsion techniques can be applied to stabilize the PVA nanoparticles. The protein containing nanoparticles has limitations on the increase of nanoparticle size upon functionalization with other nanomaterials. The protein bovine serum albumin (BSA) containing PLGA nanoparticles has good control on its size and shape while functionalizing through the solid-in-oil and oil-in-oil microsphere development method with suspended droplet hardening using impeller atomization followed by the droplet shearing in a spinning oil film approach. As shown in Figures 7 and 8, the gold nanoparticles have good interactions with polymer micelles and formed a new nanomaterial which is 
the combination of polymer micelle and gold nanoparticles with functionalized features. Similarly, iron and iron oxide containing a core of magnetite had a primary coat of a polymer of dextrans to get microspheres for bio applications [62].

However, liposomes have a spherical vesicular structure formed by the combination of hydrophilic head part and hydrophobic tail or hydrocarbon (fatty acid) chain which are called phospholipid bilayers. The hydrophilic and hydrophobic parts of the phospholipids are the main cause to form the liposome and for its structural integrity. The images in Figure 7 corroborate the monodispersed silica $\left(\mathrm{SiO}_{2}\right)$ nanoparticles prepared by using modified Stober's sol-gel method. The formation of spherical-shaped $\mathrm{SiO} 2$ nanoparticles depends on the stirring methods, the solvent used for the preparation, rotations per minute (RPM), purity of chemicals used for the synthesis of $\mathrm{SiO} 2$ nanoparticles, surfactants, and equipment used for the experiments [66]. Figure 7 shows sharp uniform-sized monodispersed silica nanoparticles are penetrating the boundary of polymer thin film and sitting inside the film.

Scientifically, it's a composite of polymer-silica, but specifically, here, in this case, polymer thin film has been inserted with the mesoporous silica nanoparticles.

Nucleic acids are another class of biopolymers which are called as polysaccharides, and they can also be involved in the development of nanocarrier capsules or particles for biomedical applications. The chemical linkage between nucleic acid monomers is phosphodiester, and it also helps in the development of nanoparticles through the self-assembly manner. The synthesized PP-based nanoparticles have precise control

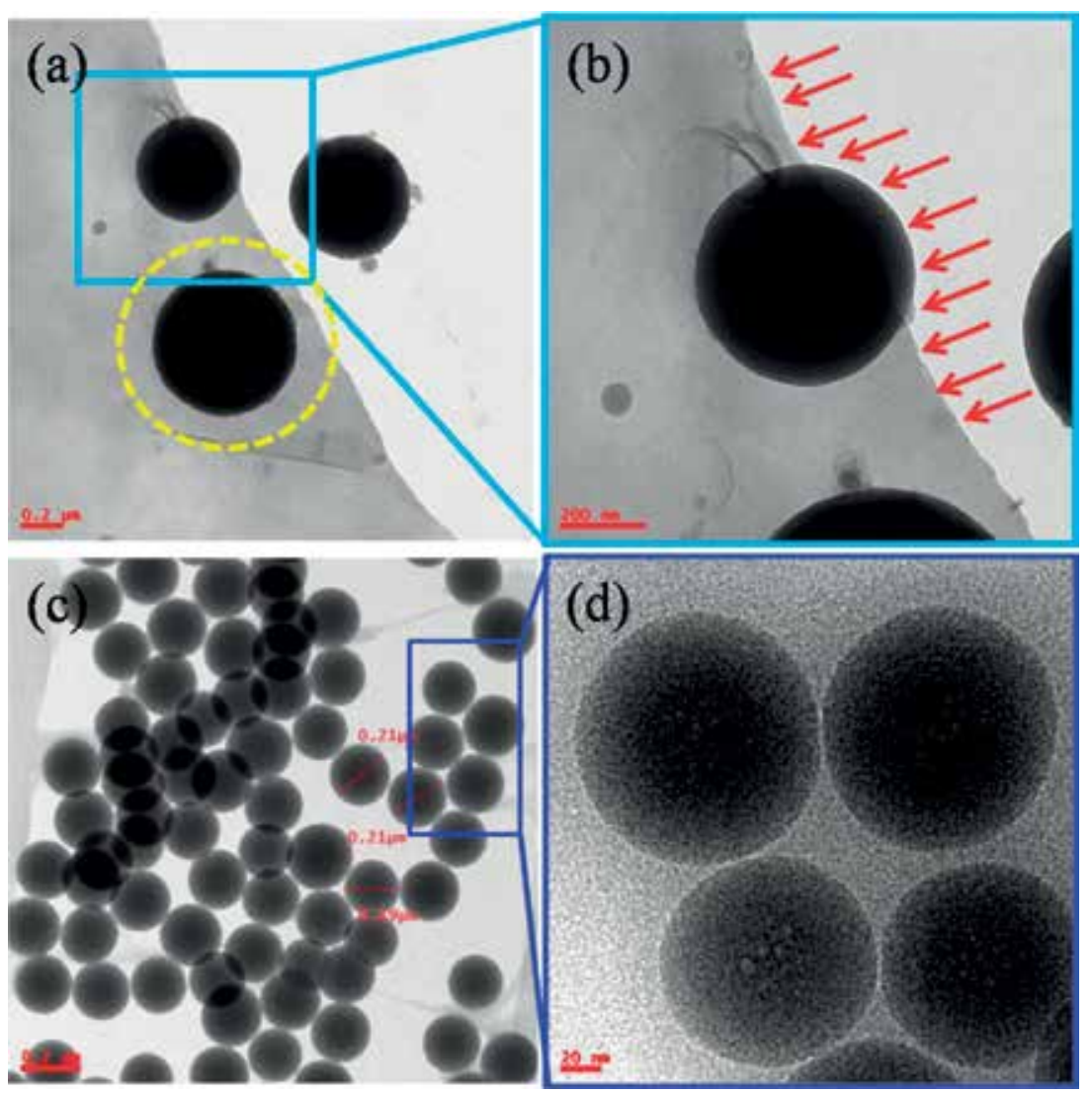

Figure 7.

Transmission electron microscopic images. $(a, b)$ Silica nanoparticle internalization into polymer thin film, (c) bare silica nanoparticles and separate polymer thin film interactions, (d) pure silica nanoparticles showing mesoscale porosity at the center and uniform size of particles at higher magnifications [62, 63]. 

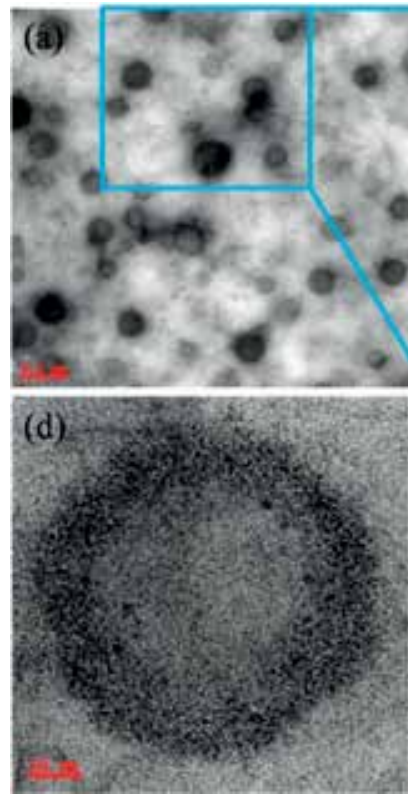

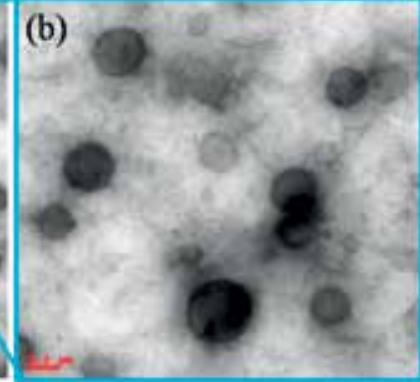

(e)

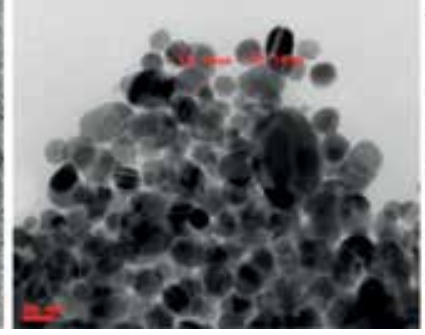

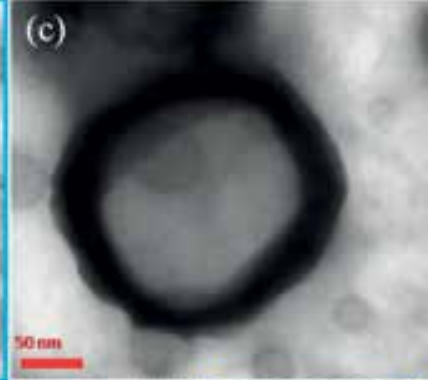

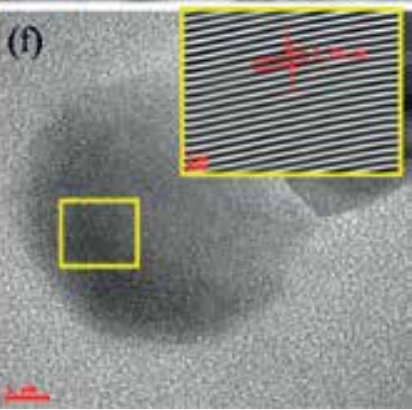

Figure 8.

Transmission electron microscopic images. $(a, b)$ Gold nanoparticles functionalized with polymer micelle, image (c) dense polymer micelle, (d) gold nanoparticles arranged in a core-shell morphology with the eternal polymer micelles, $(e)$ bare gold nanoparticles, and $(f)$ shows lattice parameters of AuNPs [64, 65].

over size and shape, and it can be controlled through the crystal sequestering of atoms and molecules [67]. Distribution of the guest particles and molecules inside the host matrix can be optimized through the agitation methods like sonication time, temperature, stirring (RPM), incubation time, and encapsulation and entrapment. However, numerous possible applications in the biomedical field arise due to the presence of long-range order in the spatial distribution of nanocarriers. Block copolymers show unique and novel characteristic features to the pattern surface of capsules and nanoparticles with porosity. The self-assembly nature between functional groups of polymers is highly dependent on solvent interactions (Figure 8). Surface and morphology of particles can be tuned based on using selective solvents. Surface patterning is an ideal as lithography templates to the spontaneous selfassembly in nanometer $(\mathrm{nm})$ to micrometer $(\mu \mathrm{m})$ size topography. The surface patterned by self-assembly of block copolymers thereby reduces the number of process steps involved in the formation of such desired structures [68].

Sometimes pore size and shape throughout the surface of the capsules and nanoparticles may appear irregular and inconsistent because of interatomic and molecular orientations and imprinting methods. However, intermolecular attractions also show the effect on the morphology of particles. There are highly inconsistent pores with smooth and rough surfaces because of the solvent effect and sonication methods. From Figure 9, AFM images of the surface profile for zinc oxide nanoparticles have been measured, and it shows the surface characters. From the image (a), the arrangement of $\mathrm{ZnONPs}$ can be seen, and the line scan profile gives a channel type of surface morphology for zinc oxide nanoparticles, whereas wet chemical methods and sample preparation procedures also play a vital role to exhibit a considerable change in shape and surface morphology of polymer NPs. A non-contact mode AFM operation has been carried out to acquire the topography of the $\mathrm{ZnONPs}$. However, tip size and magnification play a crucial role to acquire the topography of the NPs. Furthermore, for the uniform pores and consistent particle size, the shape can be optimized based on several factors such as sonication, 

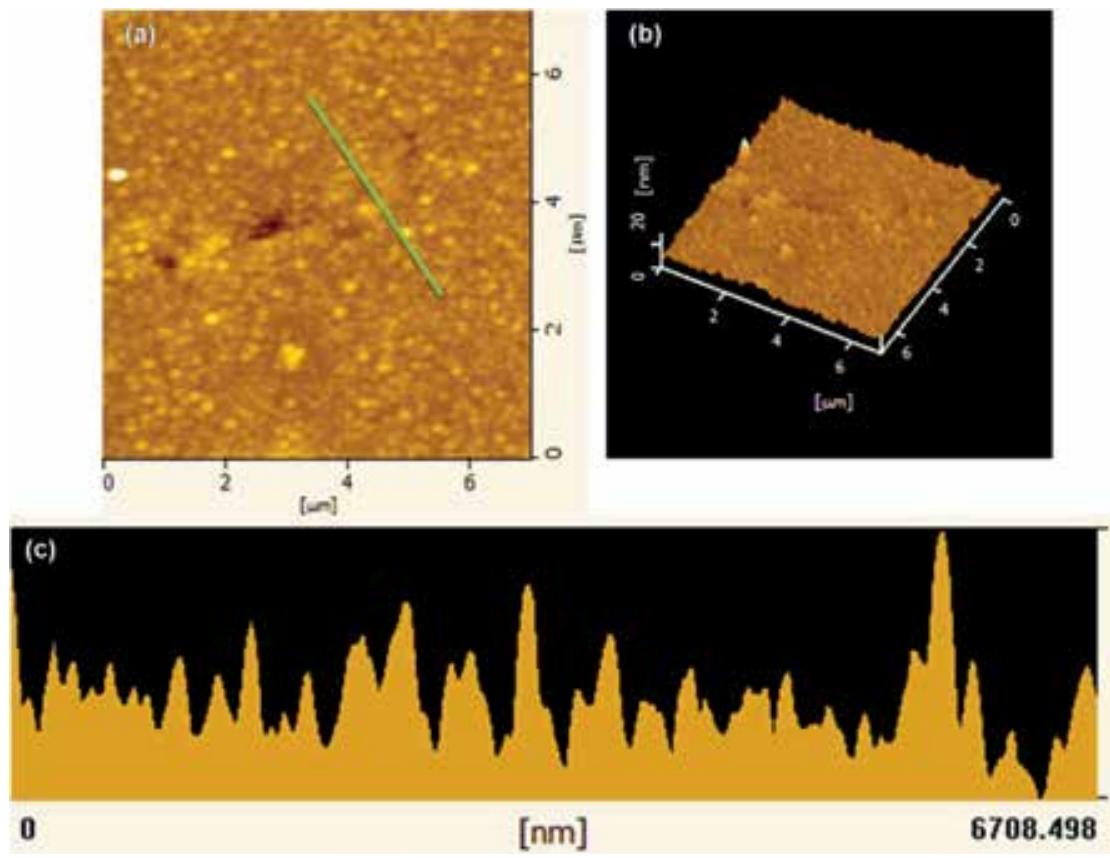

Figure 9.

Atomic force microscopic images acquired for the $\mathrm{ZnO}$ nanoparticles. The image (a) corresponds to phase(s), and it shows the surface properties of ZnO NPs, image (b) elucidates the three-dimensional view of $Z n O N P s$ and their morphology, the image (c) illustrates the surface properties of ZnONPs arranged on the substrate, and these are acquired from the line scanning mode AFM operation as represented in the image (a) phase $[64,65,69,70]$.

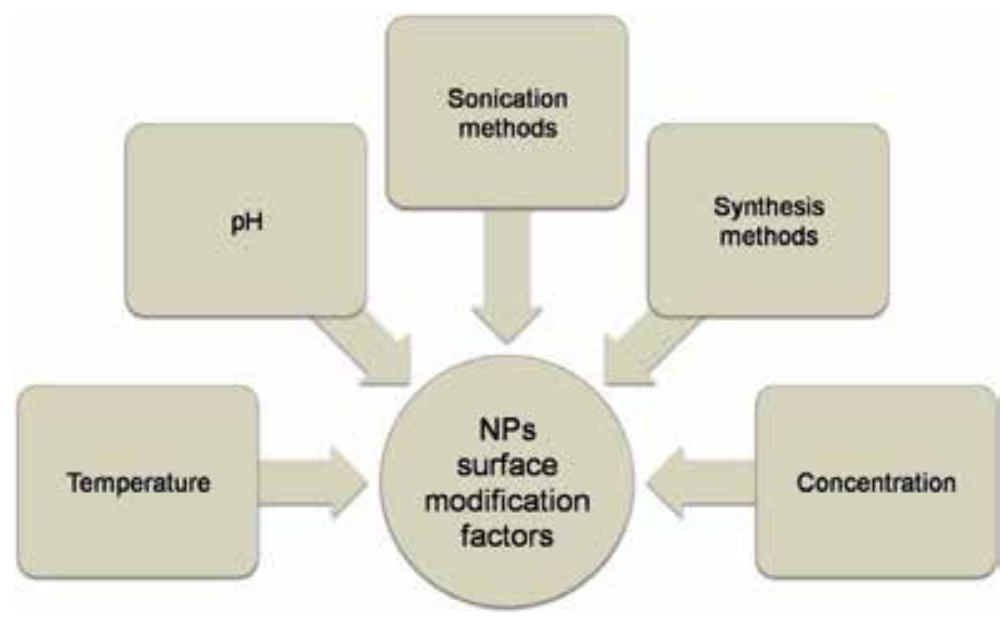

Figure 10.

Schematic shows the factors which cause the surface modification and functionalization in polymer nanoparticles.

solvent, $\mathrm{pH}$, and concentration of the system (Figure 10). The nanoscale clustering and complexation of the peripheral of nanoparticles and capsules have been done through the modification of active functional groups as potential substrates for subsequent functionalization and surface modifications. The post-insertion method is used for the preparation of dendrimer nanoparticles and lipid nanocapsules (LNCs) with the chemically reactive surface. Ideally, this method has been designed for the grafting of ligands to the polymer compounds, and later it has been 
extended to another particle development also. During the preparation of polymer nanoparticles or dendrimers, one can be focused on the experimental conditions such as the ratio between both reactants, quantity of solvent, external stimuli factors such as stirring methods, sonication, magnetic field, electric field applications, $\mathrm{pH}$, temperature, viscosity, density, and molecular weight of reactants [69].

The reaction conditions such as slow, fast, and moderate reactions can be tuned or controlled based on literature. The polymers, i.e., PCL, PLGA, PNIPAM, PS, and PEG, are advantageous to design the nanocapsules because they have the capability to form network structure which helps to load the drug molecules efficiently, and these properties help to sustain release of the medicines. The rigid morphology and close compacted structure of nanoparticles or nanocapsules with covalent bonding cannot allow us to modify its surface even at rigorous conditions also [71]. The loosely bound functional groups allow modifying the surface of the nanoparticles based on our applications. The homogeneous mixture of ingredients present in the polymer nanoparticles leads to form a close-compacted and smooth surface of the particles.

However, development of a vinyl ether-functionalized poly phosphoester as a template for multiple post-polymerization conjugation core-shell type degradable polymeric nanoparticles was appended in the various publications, and those help to design and develop polymer NPs with surface-modifiable properties [72]. The confirmation and confinement of two reactants followed by the formation nanoparticles at the interface have a unique advantage to tune their surface properties based on its phase transitions and thermal stability, and this can help to improve and to know the reaction kinetics, higher yields, and selectivity of NPs. However, the presence of a liquid interface can accelerate the reaction through the phase-transfer catalyst which is employed to draw the reaction for the development of NPs based on phase transfer phenomena. Interestingly, the use of immiscible systems in emulsions offers an easy phase separation and formulation of desired nanoparticles. However, a brief overview on low molecular weight and low-density polymer complexes show the significant proximity of the interface in emulsions, and this strategy can be used for the efficient production of nano- and microparticles for various applications [65, 69-72]. However, several mechanisms were proposed for the formation of nanoscale pores within the nanoparticles because of the removal of some of the BCP fragments from the network of the PCL. The size of the BCP-based polymer nanoparticles reduced as blocks were removed from the parent blocks. As PCL is hydrophobic in nature, therefore BCP spheroids may be dissolved out from the PCL network structure and create the pores of the equivalent size of BCP spheroids. Moreover, as per factors appended in Figure 10, temperature change affects a lot on the pore size and morphology of nanoparticles [65, 73-78].

\section{Conclusions}

Herein, polymer synthesis, design, and development of nanoparticles and their functionalization have been discussed in detail. The various polymerization methods and different types of nanoparticles and capsules were reported in this chapter. The nanoparticle/capsule classification and surface functionalization followed by size, shape, and morphological changes based on industrial and biomedical applications have been corroborated. The polymer properties play a key role in the development of NPs with desired features, and such influencing factors were reported and elaborated in the respective sections. Nanoparticles and capsules with significant changeable surface and with a more specific surface area have potential applications in various fields. The porosity on the surface of nanoparticles and capsules is an important concern to prepare the NPs for both industrial and pharmaceutical applications. 


\section{Author details}

Chander Amgoth ${ }^{1 *}$, Chiuyen Phan $^{2}$, Murali Banavoth ${ }^{3}$, Santosh Rompivalasa ${ }^{3}$ and Guping Tang ${ }^{2}$

1 Department of Sciences and Humanities, MLR Institute of Technology, Hyderabad, TS, India

2 School of Chemistry, Zhejiang University, Hangzhou, China

3 School of Chemistry, University of Hyderabad, Hyderabad, TS, India

*Address all correspondence to: chander@uohyd.ac.in

\section{IntechOpen}

(C) 2019 The Author(s). Licensee IntechOpen. This chapter is distributed under the terms of the Creative Commons Attribution License (http://creativecommons.org/licenses/ by/3.0), which permits unrestricted use, distribution, and reproduction in any medium, provided the original work is properly cited. (cc) BY 


\section{References}

[1] Villanova JC, Ayres E, Carvalho SM, Patricio PS, Pereira FV, Orefice RL. Pharmaceutical acrylic beads obtained by suspension polymerization containing cellulose nano-whiskers as an excipient for drug delivery. European Journal of Pharmaceutical Sciences. 2011;42:406-415. DOI: 10.1016/j. ejps.2011.01.005

[2] Sczech R, Riegler H. Molecularly smooth cellulose surfaces for adhesion studies. Journal of Colloid and Interface Science. 2006;301:376-385. DOI: 10.1016/j.jcis.2006.05.021

[3] Wang Y, Grayson SM. Approaches for the preparation of non-linear amphiphilic polymers and their applications to drug delivery. Advanced Drug Delivery Reviews. 2012;64:852-865. DOI: 10.1016/j.addr.2012.03.011

[4] Yaping Z, Minsheng H, Wei W, Wei J. Synthesis of the cotton cellulosebased Fe (III)-loaded adsorbent for arsenic $(\mathrm{V})$ removal from drinking water. Desalination. 2009;249:1006-1011. DOI: 10.1016/j.desal.2009.09.015

[5] Guo X, Du Y, Chen F, Park HS, Xie Y. Mechanism of removal of arsenic by bead cellulose loaded with iron oxyhydroxide ( $\beta$-FeOOH): EXAFS study. Journal of Colloid and Interface Science. 2007;314:427-433. DOI: 10.1016/j.jcis.2007.05.071

[6] Jeong B, Bae YH, Lee DS, Kim SW. Biodegradable block copolymers as injectable drug-delivery systems. Letters to Nature. 1997;388:860-862. DOI: $10.1038 / 42218$

[7] Winzenburg G, Schmidt C, Fuchs S, Kissel T. Biodegradable polymers and their potential use in parenteral veterinary drug delivery systems. Advanced Drug Delivery Reviews. 2004;56:1453-1466. DOI: 10.1016/j. addr.2004.02.008
[8] Tan Z, Xu J, Zhu T, Wang AY, Sun Q, Li Y, et al. Bright and colorsaturated quantum dot light-emitting diodes, a new star for next-generation displays and solid-state lighting. Proceedings of SPIE;7518:751803. DOI: 10.1117/12.843089

[9] Chen S, Zou Y, Yan Z, Shen W, Shi S, Zhang X, et al. Carboxymethylatedbacterial cellulose for copper and lead ion removal. Journal of Hazardous Materials. 2009;161:1355-1359. DOI: 10.1016/j.jhazmat.2008.04.098

[10] Zhou D, Zhang L, Zhou J, Guo S. Cellulose/chitin beads for adsorption of heavy metals in aqueous solution. Water Research. 2004;38:2643-2650. DOI: 10.1016/j.watres.2004.03.026

[11] Bayramoglu G, Arica MY. Kinetics of mercury ions removal from synthetic aqueous solutions using by novel magnetic p (GMA-MMA-EGDMA) beads. Journal of Hazardous Materials. 2007;144:449-457. DOI: 10.1016/j.

jhazmat.2006.10.058

[12] Jin L, Bai R. Mechanisms of Lead adsorption on chitosan/PVA hydrogel beads. Langmuir. 2002;18:9765-9770

[13] Miyajima M, Koshika A, Okada J, Ikeda M. Mechanism of drug release from a poly (L-lactic acid) matrix containing acidic or neutral drugs. Journal of Controlled Release. 1999;60:199-209

[14] Nam K, Nam HY, Kim PH, Kim SW. Paclitaxel-conjugated PEG and Arginine-grafted bioreducible poly (disulfide amine) micelles for co-delivery of drug and gene. Biomaterials. 2012;33:8122-8130. DOI: 10.1016/j.biomaterials.2012.07.031

[15] Lu Y, Chen SC. Micro and nanofabrication of biodegradable polymers for drug delivery. Advanced Drug 
Delivery Reviews. 2004;56:1621-1633.

DOI: 10.1016/j.addr.2004.05.002

[16] Yan H, Tsujii K. Potential application of poly (N-isopropylacrylamide) gel containing polymeric micelles to drug delivery systems. Colloids and Surfaces. B, Biointerfaces. 2005;46:142-146. DOI: 10.1016/j.colsurfb.2005.10.007

[17] Knop K, Hoogenboom R, Fischer D, Schubert US. Poly (ethylene glycol) in drug delivery: Pros and cons as well as potential alternatives. Angewandte Chemie, International Edition. 2010;49:6288-6308. DOI: 10.1002/ anie. 200902672

[18] Gupta S, Tyagi R, Parmar VS, Sharma SK, Haag R. Polyether-based amphiphiles for delivery of active components. Polymer. 2012;53:3053-3078. DOI: 10.1016/j. polymer.2012.04.047

[19] Yang XZ, Dou S, Wang YC, Long HY, Xiong MH, Mao CQ, et al. Single-step assembly of cationic lipid polymer hybrid nanoparticles for systemic delivery of siRNA. ACS Nano. 2012;6:4955-4965. DOI: 10.1021/ $\mathrm{nn} 300500 \mathrm{u}$

[20] Zhang Q, Xie G, Yan H. Compatibility of block copolymers on blends of polysulfone and semiaromatic polyester crystalline polymer. Polymer Plastics Technology and Engineering. 2003;42:311-326. DOI: 10.1081/ PPT-120017936

[21] Twu Y-K, Huang HI, Ying CS, Wang SL. Preparation and sorption activity of chitosan/cellulose blend beads. Carbohydrate Polymers. 2003;54:425-430. DOI: 10.1016/j. carbpol.2003.03.001

[22] Ragnarok LH, Davidson R, Nystrom C, Ragnarsson G. Pore swelling in beads made of cellulose fibers and fiber fragments. International Journal of Pharmaceutics. 1995;122:49-56
[23] Butun S, GulInce F, Erdugan H, Sahiner N. One-step fabrication of biocompatible particles carboxymethyl cellulose polymeric for drug delivery systems. Carbohydrate Polymers. 2011;86:636-643. DOI: 10.1016/j. carbpol.2011.05.001

[24] Xia HF, Lin DQ, Yao SJ. Preparation and characterization of macroporous cellulose-tungsten carbide composite beads for expanded bed applications. Journal of Chromatography. A. 2007;1175:55-62. DOI: 10.1016/j. chroma.2007.10.004

[25] O’Connell DW, Birkinshaw C, O’Dwyer TF. Heavy metal adsorbents prepared from the modification of cellulose: A review. Bioresource Technology. 2008;99:6709-6724. DOI: 10.1016/j.biortech.2008.01.036

[26] Li P, Wang Y, Peng Z, She F, Kong L. Development of chitosan nanoparticles as drug delivery systems for 5-fluorouracil and leucovorin blends. Carbohydrate Polymers. 2011;85:698704. DOI: 10.1016/j.carbpol.2011.03.045

[27] Takegawa A, Murakami M, Kaneko Y, Kadokawa J. Preparation of chitin/cellulose composite gels and films with ionic liquids. Carbohydrate Polymers. 2010;79:85-90. DOI: 10.1016/j.carbpol.2009.07.030

[28] Wolff B, Schmitz W, Schneider H. Composites of bead cellulose and hydrophilic solubilizers. International Journal of Pharmaceutics. 1996;139:87-94

[29] Bai YX, Li YF. Preparation and characterization of crosslinked porous cellulose beads. Carbohydrate Polymers. 2006;64:402-407. DOI: 10.1016/j. carbpol.2005.12.009

[30] Gurgel LV, Junior OK, Gil RP, Gil LF. Adsorption of $\mathrm{Cu}(\mathrm{II}), \mathrm{Cd}(\mathrm{II})$, and $\mathrm{Pb}$ (II) from aqueous single metal solutions by cellulose and mercerized 
cellulose chemically modified with succinic anhydride. Bioresource Technology. 2008;99:3077-3083. DOI: 10.1016/j.biortech.2007.05.072

[31] Wong AD, DeWit MA, Gillies ER. Amplified release through the stimulustriggered degradation of selfimmolation oligomers, dendrimers, and linear polymers. Advanced Drug Delivery Reviews. 2012;64:1031-1045. DOI: 10.1016/j.addr.2011.09.012

[32] Patri AK, Majoros IJ, Junior JRB. Dendritic polymer macromolecular carriers for drug delivery. Current Opinion in Chemical Biology. 2002;6:466-471. DOI: 10.1016/ S1367-5931(02)00347-2

[33] Jeong B, Bae YH, Kim SW. Drug release from biodegradable injectable thermosensitive. Journal of Controlled Release. 2000;63:155-163

[34] Svirskis D, Sejdic JT, Rodgers A, Garg S. Electrochemically controlled drug delivery based on intrinsically conducting polymers-Review. Journal of Controlled Release. 2010;146:6-15

[35] Liu T, Qi X, Huang S, Jiang L, Li J, Tang C, et al. Exploiting hydrophobic borohydride-rich ionic liquids as faster-igniting rocket fuels. Chemical Communications. 2016;52:2031-2034. DOI: $10.1039 / \mathrm{c5cc} 09737 \mathrm{a}$

[36] Tyrrell ZL, Shen Y, Radosz M. Fabrication of micellar nanoparticles for drug delivery through the selfassembly of block copolymers. Progress in Polymer Science. 2010;35:1128-1143. DOI: 10.1016/j. progpolymsci.2010.06.003

[37] Wolf B. Bead cellulose products with $\AA \operatorname{lm}$ formers and solubilizers for controlled drug release. International Journal of Pharmaceutics. 1997;156:97-107

[38] Kim H, Kim Y, Guk K, Yoo D, Lim H, Kang G, et al. Fully biodegradable and cationic poly (amino oxalate) particles for the treatment of acetaminophen-induced acute liver failure. International Journal of Pharmaceutics. 2012;434:243-250. DOI: 10.1016/j.ijpharm.2012.05.067

[39] Tencomnao T, Chaleawlert-upon S, Apijaraskul A, Rakkhithawatthana V, Pimpa N, Sajomsang W, et al. Gold/ cationic polymer nano-scaffolds mediated transfection for non-viral gene delivery system. Carbohydrate Polymers. 2011;84:216-222. DOI: 10.1016/j.carbpol.2010.12.063

[40] Liu C, Bai R. Preparation of chitosan/cellulose acetate blends hollow fibers for adsorptive performance. Journal of Membrane Science. 2005;267:68-77. DOI: 10.1016/j. memsci.2005.06.001

[41] Siepmann J, Peppas NA. Modeling of drug release from delivery systems based on hydroxypropyl methylcellulose (HPMC). Advanced Drug Delivery Reviews. 2001;48:139-157

[42] Chang C, Duan B, Cai J, Zhang L. Superabsorbent hydrogels based on cellulose for smart swelling and controllable delivery. European Polymer Journal. 2010;46:92-100. DOI: 10.1016/j.eurpolymj.2009.04.033

[43] Ramakrishna P, Mallikarjuna B, Chandra Babu A, Sudhakar P, Rao KC, Subha MCS. Interpenetrating polymer network of crosslinked blend microspheres for controlled release of Acebutolol HCl. Journal of Applied Pharmaceutical Science. 2011;1:212-219

[44] Liang S, Zhang L, Xu J. Morphology and permeability of cellulose/chitin blend membranes. Journal of Membrane Science. 2007;287:19-28. DOI: 10.1016/j. memsci.2006.10.002

[45] Wang Y, Zhao Q, Han N, Bai L, Li J, Liu J, et al. Mesoporous silica nanoparticles in drug delivery and 
biomedical applications. Nanomedicine: Nanotechnology, Biology, and Medicine. 2015;11:313-327. DOI: 10.1016/j. nano.2014.09.014

[46] Husseini GA, Pitt WG. Micelles and nanoparticles for ultrasonic drug and gene delivery. Advanced Drug Delivery Reviews. 2008;60:1137-1152. DOI: 10.1016/j.addr.2008.03.008

[47] Lukyanov AN, Torchilin VP. Micelles from lipid derivatives of water-soluble polymers as delivery systems for poorly soluble drugs. Advanced Drug Delivery Reviews. 2004;56:1273-1289. DOI: 10.1016/j. addr.2003.12.004

[48] Cunliffe D, Kirby A, Alexander C. Molecularly imprinted drug delivery systems. Advanced Drug Delivery Reviews. 2005;57:1836-1853. DOI: 10.1016/j.addr.2005.07.015

[49] Efentakis M, Politis S. Comparative evaluation of various structures in polymer-controlled drug delivery systems and the effect of their morphology and characteristics on drug release. European Polymer Journal. 2006;42:1183-1195. DOI: 10.1016/j. eurpolymj.2005.11.009

[50] Wang T, Li M, Gao H, $\mathrm{Wu}$ Y. Nanoparticle carriers based on copolymers of poly (e-caprolactone) and hyperbranched polymers for drug delivery. Journal of Colloid and Interface Science. 2011;353:107-115. DOI: 10.1016/j.jcis.2010.09.053

[51] Yan W, Hsiao VKS, Zheng YB, Shariff YM, Gao T, Huang TJ. Towards nanoporous polymer thin film-based drug delivery systems. Thin Solid Films. 2009;517:1794-1798. DOI: 10.1016/j. tsf.2008.09.080

[52] Vashist A, Gupta YK, Ahmad S. Interpenetrating biopolymer network-based hydrogels for an effective drug delivery system. Carbohydrate Polymers. 2012;87:1433-1439. DOI: $10.1016 / \mathrm{j}$. carbpol.2011.09.030

[53] Malafaya PB, Silva GA,

Reis RL. Natural-origin polymers as carriers and scaffolds for biomolecules and cell delivery in tissue engineering applications. Advanced Drug Delivery Reviews. 2007;59:207-233. DOI: 10.1016/j.addr.2007.03.012

[54] Giavaresi G, Tschon M, Borsari V, Daly JH, Liggat JJ, Fini M, et al. New polymers for drug delivery systems in orthopedics: in vivo biocompatibility evaluation. Biomedicine \& Pharmacotherapy. 2004;58:411-417. DOI: 10.1016/j.biopha.2004.08.001

[55] Cypes SH, Saltzman WM, Giannelis EP. Organosilicate-polymer drug delivery systems: Controlled release and enhanced mechanical properties. Journal of Controlled Release. 2003;90:163-169. DOI: 10.1016 / S0168-3659(03)00133-0

[56] Sadekar S, Ghandehari H.

Transepithelial transport and toxicity of PAMAM dendrimers: Implications for oral drug delivery. Advanced Drug Delivery Reviews. 2012;64:571-588. DOI: 10.1016/j.addr.2011.09.010

[57] Rackstraw BJ, Stolnik S, Davis SS, Bignotti F, Garnett MC. Development of multicomponent DNA delivery systems based upon poly (amidoamine)-PEG copolymers. Biochimica et Biophysica Acta. 2002;1576:269-286

[58] Nakamura K, Nara E, Akiyama Y. Development of an oral sustained release drug delivery system utilizing $\mathrm{pH}$-dependent swelling of a carboxyvinyl polymer. Journal of Controlled Release. 2006;111:309-315. DOI: 10.1016/j.jconrel.2005.12.018

[59] Crowley MM, Schroeder B, Talarico M, Fredersdorf A, Obara S, Kucera S, et al. Physicochemical 
properties and mechanism of drug release from ethyl cellulose matrix tablets prepared by direct compression and hot-melt extrusion. International Journal of Pharmaceutics. 2004;269:509-522. DOI: 10.1016/j. ijpharm.2003.09.037

[60] Kabanov AV, Batrakova EV, Alakhov VY. Pluronic block copolymers as novel polymer therapeutics for drug and gene delivery. Journal of Controlled Release. 2002;82:189-212

[61] Li G, Liu J, Pang Y, Wang R, Mao L, Yan D, et al. Polymeric micelles with water-insoluble drug as hydrophobic moiety for drug delivery. Biomacromolecules. 2011;12:2016-2026. DOI: $10.1021 / \mathrm{bm} 200372 \mathrm{~s}$

[62] Gong J, Chen M, Zheng Y, Wang S, Wang Y. Polymeric micelles drug delivery system in oncology. Journal of Controlled Release. 2012;159:312-323. DOI: 10.1016/j. jconrel.2011.12.012

[63] Amgoth C, Suman J. Thermosensitive block copolymer [(PNIPAM)-b(glycine)] thin film as a protective layer for drug-loaded mesoporous silica nanoparticles. Materials Research Express. 2017;4:105306. DOI: 10.1088/2053-1591/aa91eb

[64] Amgoth C, Dharmapuri G, Kalle AM, Paik P. Nanoporous capsules of block copolymers of [(MeOPEG-NH)-b-(L-GluA)]-PCL for the controlled release of anticancer drugs for therapeutic applications. Nanotechnology. 2016;27:125101. DOI: $10.1088 / 0957-4484 / 27 / 12 / 125101$

[65] Amgoth C, Suman Joshi DSD, Dharmapuri G, Mohan Lakavathu M. Self-assembled block copolymer [(BenzA)-b-(PCL)] micelles to orient randomly distributed AuNPs into hollow core-shell morphology and its role as payload for nanomedicines. Materials Science and Engineering: C.
2018;92:790-799. DOI: 10.1016/j. msec.2018.07.033

[66] Prakash S, Malhotra M, Shao W, Duchesneau CT, Abbasi S. Polymeric nanohybrids and functionalized carbon nanotubes as drug delivery carriers for cancer therapy. Advanced Drug Delivery Reviews. 2011;63:1340-1351. DOI: 10.1016/j.addr.2011.06.013

[67] Patel T, Zhou J, Piepmeier JM, Saltzman WM. Polymeric nanoparticles for drug delivery to the central nervous system. Advanced Drug Delivery Reviews. 2012;64:701-705. DOI: 10.1016/j.addr.2011.12.006

[68] Agarwal RC, Kant S, Singh PN, Maiti P, Pandit JK. Polymeric nanoparticulate system: A potential approach for ocular drug delivery. Journal of Controlled Release. 2009;136:2-13. DOI: 10.1016/j. jconrel.2008.12.018

[69] Oster J, Parker J, Brassard L. Polyvinyl-alcohol-based magnetic beads for rapid and efficient separation of specific or unspecific nucleic acid sequences. Journal of Magnetism and Magnetic Materials. 2001;225:145-150

[70] Sarparanta MP, Bimbo LM, Makila EM, Salonen JJ, Laaksonen PH, Helariutta AMK, et al. The mucoadhesive and gastro-retentive properties of hydrophobin-coated porous silicon nanoparticle oral drug delivery systems. Biomaterials. 2012;33:3353-3362. DOI: 10.1016/j. biomaterials.2012.01.029

[71] Whittlesey KJ, Shea LD. Delivery systems for small molecule drugs, proteins, and DNA: The neuroscience/ biomaterial interface. Experimental Neurology. 2004;190:1-16. DOI: 10.1016/j.expneurol.2004.06.020

[72] Yun J, I'm JS, Lee YS, Kim HI. Electro-responsive transdermal drug delivery behavior of PVA/PAA/ 
MWCNT nanofibers. European Polymer Journal. 2011;47:1893-1902. DOI: 10.1016/j.eurpolymj.2011.07.024

[73] Muhlen AZ, Schwarz C, Mehnert W. Solid lipid nanoparticles (SLN) for controlled drug deliveryDrug release and release mechanism.

European Journal of Pharmaceutics and Biopharmaceutics. 1998;45:149-155

[74] Ramesh G, Dharmapuri G, Suman Joshi D, Amgoth C. Composite BMEAuNPs: Chemopreventive effect on skin carcinoma and inhibition on leukemia blood cancer cells. Advanced Materials Letters. 2017;8:1057-1064. DOI: 10.5185/ amlett.2017.1733

[75] Amgoth C, Dharmapuri G. Synthesis and characterization of polymeric nanoparticles and capsules as payload for anticancer drugs and nanomedicines. Materials Today: Proceedings. 2016;3:3833-3837

[76] Paik P, Manda R, Amgoth C, Santhosh KK. Polyaniline nanotubes with rectangular-hollow-core and its self-assembled surface decoration: High conductivity and dielectric properties. RSC Advances. 2014;4:12342. DOI: 10.1039/c3ra47155a

[77] Doddapaneni SJDS, Amgoth C, Kalle AM, Suryadevara SN, Alapati KS. Antimicrobial and anticancer activity of AgNPs coated with Alphonsea sclerocarpa extract. 3 Biotech. 2018;8:156. DOI: 10.1007/s13205-018-1155-9

[78] Liang Y, Deng L, Chen C, Zhang J, Zhou R, Li X, et al. Preparation and properties of thermoreversible hydrogels based on methoxy poly (ethylene glycol)-grafted chitosan nanoparticles for drug delivery systems. Carbohydrate Polymers. 2011;83:1828-1833. DOI: 10.1016/j. carbpol.2010.10.048 


\title{
Applications of Chitosan in Pulmonary Drug Delivery
}

\author{
Xiuwen Guan and Weifen Zhang
}

\begin{abstract}
Pulmonary administration is an effective method for treating lung and other diseases. Drugs can be transported directly to the lung by the pulmonary drug delivery system (PDDS). PDDS has the advantages of maintained local drug concentration, reduced side effects, controllable drug release, promoted drug absorption, prolonged drug action time, and improved patient compliance. Polymers have been extensively utilized to prepare novel PDDS. Among these polymers, chitosan (CS) is a natural cationic polysaccharide which is rich in its source. It has many unique physicochemical properties, good biocompatibility, and satisfactory biodegradability. CS has been a popular biomaterial in pharmaceutics for decades and is widely used in drug delivery. CS contains many amino groups. The contained positive charges can interact strongly with the negatively charged mucosa membranes, thereby facilitating CS adsorption on the mucosal surface, avoiding clearance by the cilia, and improving the adhesion and penetration rate on the cell membrane. Moreover, studies have shown that CS could open cell tight junctions, which would promote drug transportation across the epithelial tissue. Thus, CS is an especially suitable material for PDDS. In this chapter, we will focus on the research progress and the applications of CS in PDDS. Many representative and advanced studies on CS-based PDDS are reviewed in detail.
\end{abstract}

Keywords: chitosan, pulmonary drug delivery, microsphere, nanoparticle, liposome, targeted delivery

\section{Introduction}

In recent decades, with the continuous development of medical technology, people have achieved an in-depth understanding of lung functions and characteristics. Pulmonary administration has been recognized as a simple and effective method for treating lung and other diseases [1-3]. The drug delivery system (DDS), which transports drugs directly to the lung to produce local or systemic therapeutic effects, is known as the pulmonary drug delivery system (PDDS). Oral drugs need to be absorbed by the gastrointestinal tract and further transported through the blood circulation to reach the lesion site. In this series of complex processes, serious drug loss happens after confronting acid, alkali, enzyme degradation, and liver elimination. Ultimately, only a small amount of drugs can reach the lesion site, which seriously reduces the drug bioavailability and impairs the therapeutic outcomes [4]. Parenteral administration can cause damage to the tissue and reduce patient compliance. By contrast, PDDS can deliver the drugs directly to the lung 
through the trachea for the local treatment of lung diseases. Compared with systemic administration, pulmonary administration is an ideal way for treating lung disease. It can significantly reduce the dosage, and decrease the drug distribution in nontarget tissues, thereby reducing the toxicity and side effects [5]. After administration through the lung, the drugs can be transported into the systemic circulation through the thin alveolar epithelial cell layer. Thus, PDDS can also be used for systemic drug delivery. Due to the large absorption area of the lung and the lack of degrading enzymes, pulmonary administration can be used as a convenient and effective noninjection administration method for biomacromolecular drugs, such as proteins and nucleic acids [6-8]. The PDDS can maintain local drug concentration, reduce systemic side effects, control drug release, promote drug absorption, prolong the drug action time, and improve patient compliance. Therefore, PDDS has become a hotspot to prevent and treat diseases in current research $[9,10]$.

Chitosan (CS) is a natural and widely available polycationic polysaccharide, and it has many unique physicochemical properties, good biocompatibility, and satisfactory biodegradability [11]. CS has been a popular biomaterial in pharmaceutical research for decades and is widely used in drug delivery [12]. As a drug carrier, CS can control drug release and improve the dissolution of poorly soluble drugs. A large number of studies have reported the developments and applications of CS-based materials as drug delivery carriers with versatile functions, such as CS-based beads, films, sponges, hydrogels, microspheres, and nanoparticles (NPs) [13]. Moreover, $\mathrm{CS}$ contains a large number of positively charged amino groups. These positive charges can interact strongly with the negatively charged mucosa membranes and adsorb on the mucosal surface, thereby avoiding the drugs being removed by the cilia, improving the adhesion rate, reducing drug clearance, and providing conditions for the drugs to penetrate through the cell membrane. At the same time, studies have shown that CS can open the tight junctions between the cells, which will promote drug transportation through the epithelial tissues, and increase the drug absorption rate and bioavailability [14]. Thus, CS is a suitable material especially for PDDS.

In this chapter, we focus on the applications of CS in pulmonary drug delivery. The research progress of PDDS and the applications of CS in PDDS are reviewed, and many representative and advanced studies on CS-based PDDS are enumerated in detail.

\section{The research progress of PDDS}

\subsection{The physiological basis of pulmonary administration}

Lung is the respiratory organ of the human body. It cooperates with the trachea to constitute the main place for gas exchange. The bronchi and lung can provide a large absorption area for drugs, and the total number of human alveoli is as high as $5.6 \times 10^{8}$, and the total surface absorption area can reach up to $140 \mathrm{~m}^{2}$ [15]. Moreover, the distance from the alveoli surface to the capillaries is only about $1 \mu \mathrm{m}$. By contrast, the distance is about $40 \mu \mathrm{m}$ from the microvilli of the small intestine mucosa to the capillaries. Therefore, the transport distance required for the pulmonary absorption process is much shorter than that of intestinal absorption, and the drugs can be rapidly absorbed through pulmonary administration. At the same time, the lung contains the most abundant capillaries compared with other organs in human, and about $90 \%$ of the alveolar area is covered with capillaries. The area of pulmonary capillaries in adults can be as wide as $80 \mathrm{~m}^{2}$. Moreover, the volume of blood flowing through the lung is very high, as almost all of the blood discharged 
from the right ventricle will pass through the lung, which is up to $5 \mathrm{~L}$ per minute. This blood flow volume is the equivalent of the total blood flow in all other organs and tissues of the body [16]. Besides, the chemical and enzymatic degradation activity in the lung is relatively low, which can reduce the hydrolysis of the bioactive macromolecules, such as proteins, peptides, and nucleic acids. Thus, these drugs can maintain a good biological activity after pulmonary administration, which will be beneficial to improve the drug bioavailability.

In summary, the huge absorption area, abundant capillaries, and minimal transport distance together contribute to the rapid absorption of pulmonary administration. After being quickly absorbed in the lung, the drugs will directly enter the systemic blood circulation, avoiding the first-pass effect of the liver, which is beneficial to improve the bioavailability of the drugs. And due to the low enzyme activity in the lung, the drug's adverse reactions during local administration can be reduced, which is especially suitable for the patients who need long-term administration of the medicine [17-19].

\subsection{The characteristic and development of pulmonary administration}

Pulmonary administration has been initially used to relax the tracheal smooth muscles to treat the acute exacerbation of asthma, and the disease is treated by inhaling the drugs through the trachea and the lung in the forms of drug aerosol particles or dry powder particles [20]. In general, there are two therapeutic purposes for pulmonary administration. One is the treatment of diseases in the lung, such as bronchial asthma and chronic obstructive pulmonary disease. The other purpose is to achieve systemic treatment through the pulmonary absorption of the drugs. Pulmonary inhalation administration can deliver the therapeutic agents directly to the lesion site, which can reduce the drug distribution in nontarget tissues. Therefore, for the treatment of pulmonary diseases, inhalation administration has a higher therapeutic index and fewer side effects than oral administration. And pulmonary inhalation is the preferred administration method for bronchodilators, $\beta 2$-receptor agonists and corticosteroids [21].

With the deep understanding of lung functions and lung diseases in medicine field, pulmonary inhalation is recognized as a simple and effective administration route for the respiratory tract and other disease treatments. The types of the pulmonary inhalation-treated diseases have been gradually increasing, such as the insulin aerosol for treating diabetes, and the salmon calcitonin powder for the treatment of osteoporosis and osteoarthritis [22]. In recent years, the number of studies on pulmonary inhalation of macromolecular drugs, such as proteins and peptides, has been increasing. These macromolecular drugs (such as insulin, growth hormone, vaccines, and cytokines) can be formulated into pulmonary administration preparations for local or systemic treatment. However, most of the pulmonary inhalation drugs currently used in clinical treatment is short-acting preparations, which require frequent administrations (about 3-4 times a day). Therefore, the long-acting preparations should be developed, because they can maintain stable blood concentrations and also increase the compliance of the patients [23]. At the same time, the sustained or controlled drug release preparations for pulmonary administration can effectively regulate drug release behavior and promote drug absorption rate, thereby contributing to the achievement of ideal therapeutic effects [10].

The particle size of the pulmonary inhalation preparations directly affects the deposition form and deposition site in the lung [24-26]. The particles of sizes $>5.0 \mu \mathrm{m}$ produce inertial impact and are deposited in the pharynx, larynx, and upper respiratory tract. Particles of sizes 1.0-5.0 $\mu \mathrm{m}$ mainly reach the deep part of the respiratory tract, trachea, bronchi, and alveolar surface by gravity deposition. 
The particles of sizes $0.5-1.0 \mu \mathrm{m}$ are deposited on the respiratory bronchioles and alveolar walls. The particles of sizes $<0.5 \mu \mathrm{m}$ will be discharged out with airflow due to the Brownian motion, and typically $80 \%$ of them will be expelled out of the respiratory tract. Therefore, the particles with a size range of 1.0-5.0 $\mu \mathrm{m}$ have the highest deposition rate in the bronchioles and alveoli, and they are generally selected as the main components of the pulmonary inhalation preparations. Studies have shown that the pulmonary absorption of the drugs is a passive process, a small molecular weight contributes to fast drug absorption, and the absorption of the macromolecular drug is relatively slow [16]. The drugs with molecular weight below 1000 Da present short absorption half-life and good bioavailability. As the alveolar wall is very thin, macromolecular drugs can also be absorbed through the large gap between the cells or be swallowed into the lymphatic system by the macrophages in alveoli, before finally entering the blood circulation [27].

In recent years, with the development of biomaterial science, biotechnology, and medical technology, the research of new PDDS for drug-loading has focused on polymer-based microspheres, liposomes, and NPs, which can be inhaled into the lung and deposited in the lung mucosa through atomization and in the form of dry powder or other forms [28]. Compared with the atomized injections currently used in clinic, the pulmonary administration preparations based on new PDDS have the advantages of convenience, sustained or controlled drug release, prolonged drug action time, enhanced bioavailability, and improved therapeutic efficiency. PDDS with better efficacy will be designed with the development of new materials and the advancement of pharmaceutical preparation technologies. Pulmonary administration will have broad prospects for disease treatment in the medical field.

\section{The applications of CS in PDDS}

\subsection{The basic properties of CS}

CS is a nontoxic natural polymer that is the only basic polysaccharide found in nature. It has good physical and chemical properties. CS has many advantages, such as widely available, nontoxic, biocompatible, biodegradable, and structure modifiable, and its derivatives also have some unique properties. CS has been widely used in environmental engineering, textile industry, papermaking, food industry, cosmetics, medicine, biotechnology, pharmacy, and also many other fields [29, 30]. In recent years, with the rapid development of the advanced DDS, CS and its derivatives have received extensive attention as drug carrier materials, especially for sustained and controlled drug release, and they have become popular research topics in this field [31, 32].

As a drug carrier, CS has the following advantages compared with other materials. (1) CS contains a large amount of amino groups, which easily become positively charged via combination with the $\mathrm{H}^{+}$in solution. Thus, CS has a strong adsorption effect on carrying drugs with different characteristics [33, 34]. (2) The polysaccharide chain of the CS and the lipopolysaccharide (LPS) structure can be recognized by the macrophages, thereby activating and triggering local immune responses and enhancing the targeting effect to specific tissues or cells [35]. (3) CS and its derivatives have good antibacterial activity and can inhibit the growth and reproduction of some fungi, bacteria, and viruses [36]. (4) CS is a polycation, which can easily interact with the negatively charged groups on the cell membrane surface, thereby changing the fluidity and permeability of the cell membrane [37]. (5) CS is especially suitable for local administration because of its good adhesion property and histocompatibility, which can particularly meet the requirements for drug 
retention after mucosal administration [38]. Therefore, CS has a wide range of applications in drug delivery. It can increase the stability of the drug, prolong the drug action time, change the administration route, increase the targeting ability of the drug, control the drug release, improve the dissolution of drugs with poor solubility, and adjust the cell membrane permeability of the hydrophobic drugs. At the same time, the positively charged CS can be easily adsorbed on the mucosal surface and also hard to be removed by the cilium, thereby providing conditions for the drug to penetrate the cell membrane. Moreover, CS can open the tight junctions between the cells, which will promote drug transportation in the epithelial tissues and increase the drug absorption rate and bioavailability. Thus, CS is especially applicable for PDDS [39, 40]. CS also has inherent immunogenicity, which is absent in other polymers, and this enables its use as an adjuvant for vaccine delivery into the lung [5]. Therefore, research on the applications of the CS-based PDDS has attracted great attention all over the world.

\subsection{Novel PDDS based on CS and its derivatives}

Traditional pulmonary administration preparations have drawbacks such as relatively short drug onset time, high frequency of administration, and poor patient compliance. In order to overcome these problems, research has been focused on the development of new PDDS with sustained or controlled drug release properties, also with active targeting abilities, for increasing the drug retention time in the lung, improving the drug concentration in treated areas, reducing the damage to normal tissues or cells, and enhancing the bioavailability of the drugs. The new formulations for PDDS in recent studies mainly include microspheres, polymeric NPs, liposomes, and active targeted systems [41]. In the following content, we will introduce the above-mentioned formulations one by one.

\subsubsection{CS-based microspheres}

Microspheres are microparticulate disperse systems formed by drugs dispersed or adsorbed in the polymer matrix. Microspheres have some unique advantages as a DDS for pulmonary administration [42]. They can be deposited in the lung, delay the drug release, and protect biomacromolecules, such as proteins and peptides from hydrolysis by enzymes. By optimization of the preparation process, a microsphere with an aerodynamic diameter of 1-5 $\mu \mathrm{m}$ and with suitable shape and porosity can be obtained, for meeting the requirements of pulmonary administration. In addition, microspheres usually have good stability with high moisture resistance ability. These characteristics have determined the wide applications of microspheres in pulmonary administration formulations [43].

There are many kinds of carrier materials for preparing microspheres. At present, the use of biodegradable microsphere as controlled release carrier is popular in DDS research [44]. Poly (lactic-co-glycolic acid) copolymer (PLGA) and CS are the commonly used biodegradable materials for microsphere preparation [45]. The conventional methods for preparing CS microspheres include emulsion crosslinking, solvent evaporation, ion induction, and spray drying [46-49]. Among these, crosslinking is the most commonly used method in the preparation of drugloaded CS microspheres with controlled-release property. The reaction can be carried out under mild conditions, and it also can be industrially prepared easily [50]. Moreover, as CS is positively charged, it can combine with the negatively charged drugs by electrostatic binding interaction to form a complex, which can help to improve the drug loading capacity of the microspheres. 
Weifen Zhang's group had developed a series of CS-based microspheres for pulmonary administration. They reported the preparation of CS and $\beta$-cyclodextrin microspheres as PDDS [51-55]. The microspheres were prepared by the spray drying method, and theophylline was loaded into the microspheres as a model drug. These microspheres possessed spherical shape with smooth or wrinkled surfaces, and had suitable aerodynamic diameters, which were suitable for inhalation. The microspheres had high drug entrapment and encapsulation efficiency. They can remain stable under storage conditions. The microspheres could also easily penetrate the membrane with a high permeation rate. The results showed that these microspheres had good potential as a sustained drug release carrier for pulmonary administration.

In Weifen Zhang's another work, paclitaxel (PTX) and quercetin (QUE) were respectively loaded in the NPs, which were synthesized with the oleic acidconjugated CS (OA-CTS). And these drug-loaded NPs were further used in the preparation of polymeric microspheres (PMs) by the spray-drying method (Figure 1) [56]. The microspheres could help prolong the retention time of PTX in the presence of QUE, for bypassing the P-glycoprotein drug efflux pumps. The diameters of the PMs ranged from 1 to $5 \mu \mathrm{m}$, and they had a uniform size distribution. The PMs displayed slow-release characteristics at $\mathrm{pH}$ levels of 4.5 and 7.4. In vivo pharmacokinetic and biodistribution studies suggested that the PMs exhibited a prolonged circulation time and a markedly high accumulation in the lung. The PMs could serve as a promising PDDS for combined therapy using hydrophobic drugs.

Recently, Ludmylla Cunha et al. developed inhalable CS microparticles for simultaneous delivery of isoniazid and rifabutin in lung tuberculosis treatment [57]. Spray-dried CS microparticles were obtained with adequate flow properties for deep lung delivery (aerodynamic diameter of $4 \mu \mathrm{m}$ ) and high drug association efficiencies (93\% for isoniazid and $99 \%$ for rifabutin). No cytotoxicity effect was found in human alveolar epithelial (A549) cells. The CS microparticles could activate macrophage-like cells, inducing cytokine secretion well above basal levels. Moreover, the uptake level of macrophages to internalize microparticles was over $90 \%$. The microparticles also inhibited bacterial growth by $96 \%$, demonstrating that the microencapsulation preserved drug antibacterial activity in vitro. The dual drug-loaded CS microparticles demonstrated to be potential candidates for inhalable therapy of pulmonary tuberculosis.

\subsubsection{CS-based NPs}

As a potential DDS, NPs have been widely used in medicine and other fields, and it has already become a research hotspot for decades [58-60]. NPs can improve the solubility and stability of the drugs, prolong the half-life, and enhance the drug absorption rate. NPs can also help to realize sustained or controlled drug release, prolong drug acting time, reduce administration frequency, and improve patient compliance. Moreover, NPs can target the drugs to specific organs and cells by the passive or active targeting ability of the multiple functionalized NPs. The NPs can be modified to avoid the phagocytosis of the macrophages or the removal of mucosal cilia, thereby improving the bioavailability of the drugs.

Drug delivery by NPs is an effective approach for the pulmonary administration of insoluble drugs $[61,62]$. The surface of the NPs can be modified to prolong the drug residence time in the lung and to achieve appropriate release property for improving the therapeutic effect. The pulmonary administration route for NPs is mostly by inhalation in the form of aerosolized colloidal solution. However, when the NPs are administered directly into the lung, some of them may be discharged out with the breath due to their small particle size, thereby resulting in low 
A
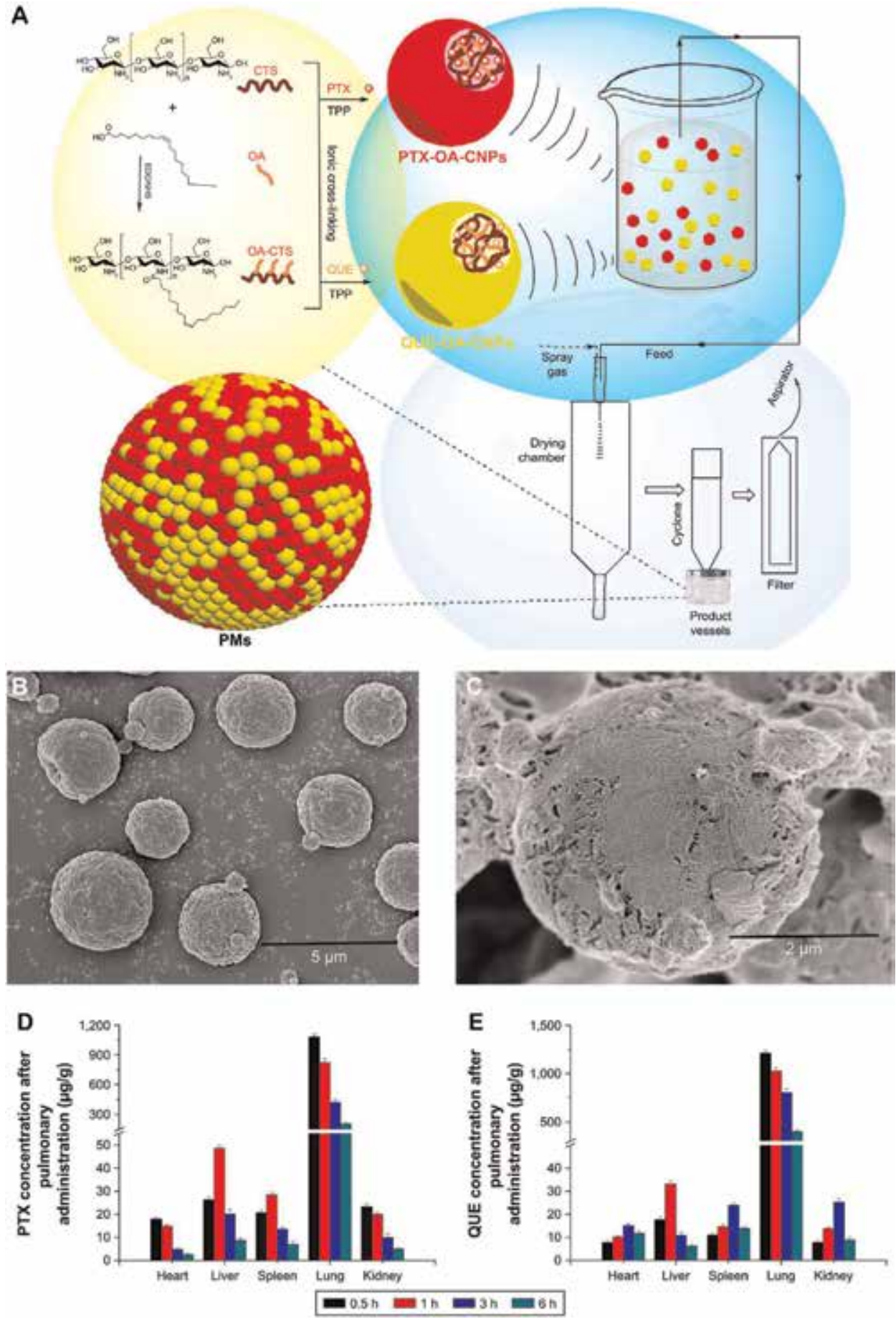

Figure 1.

(A) The synthesis and preparation route of the PMs. (B and C) SEM image of the PMs with a scale bar of 5 and $2 \mu \mathrm{m}$. (D and E) The concentration of PTX and QUE accumulated in different organs measured by HPLC at $0.5,1,3$, and 6 h after pulmonary administration.

deposition in the lung and discounted effect. Studies have shown that coating the surface of the NPs with biocompatible polymers, such as CS, can prolong the residence time of NPs in the lung [5]. Sometimes the surface energy of the NPs is 
relatively high, and the weak stability will lead to aggregations and interactions between the NPs. Thus, special surface modification will also be an effective way to improve the stability of the NPs.

Kenneth A. Howard et al. had developed CS-based siRNA-loaded NPs for pulmonary RNA interference therapy [63]. The negatively charged siRNA was complexed by the positively charged CS to form the polyelectrolyte complex NPs. The particle size ranged from 40 to $600 \mathrm{~nm}$. These NPs showed a rapid uptake (1 h) into NIH 3T3 cells. The NPs could mediate efficient knockdown of endogenous enhanced green fluorescent protein (EGFP) in both $\mathrm{H} 1299$ human lung carcinoma cells and murine peritoneal macrophages in vitro. Moreover, effective in vivo RNA interference was also realized in the bronchiole epithelial cells of transgenic EGFP mice after nasal administration. The results indicated that this kind of CS-based complex NPs has great potential in RNA interference therapy for systemic and mucosal disease.

A series of CS/fucoidan (CS/F) NPs had been designed and prepared as PDDS for gentamicin (GM) in Yi-Cheng Huang's study (Figure 2) [64]. The NPs presented a biphasic release feature, with a zero-order release of GM for the first $10 \mathrm{~h}$, followed by a sustained release of up to $72 \mathrm{~h}$, and the cumulative release value reached 99\%. The GM-loaded CS/F NPs exhibited multiple antimicrobial capabilities against Klebsiella pneumoniae. The intratracheal administration of the GMloaded CS/F NPs displayed a higher area under the curve (AUC) and lower minimum inhibitory concentration ratio than the free GM that was administrated by intravenous injection. These results had showed that the CS/F NPs had superior antimicrobial efficacy and lower risk of systemic toxicity, and this GM pulmonary delivery system has exhibited good potential for pneumonia treatment.
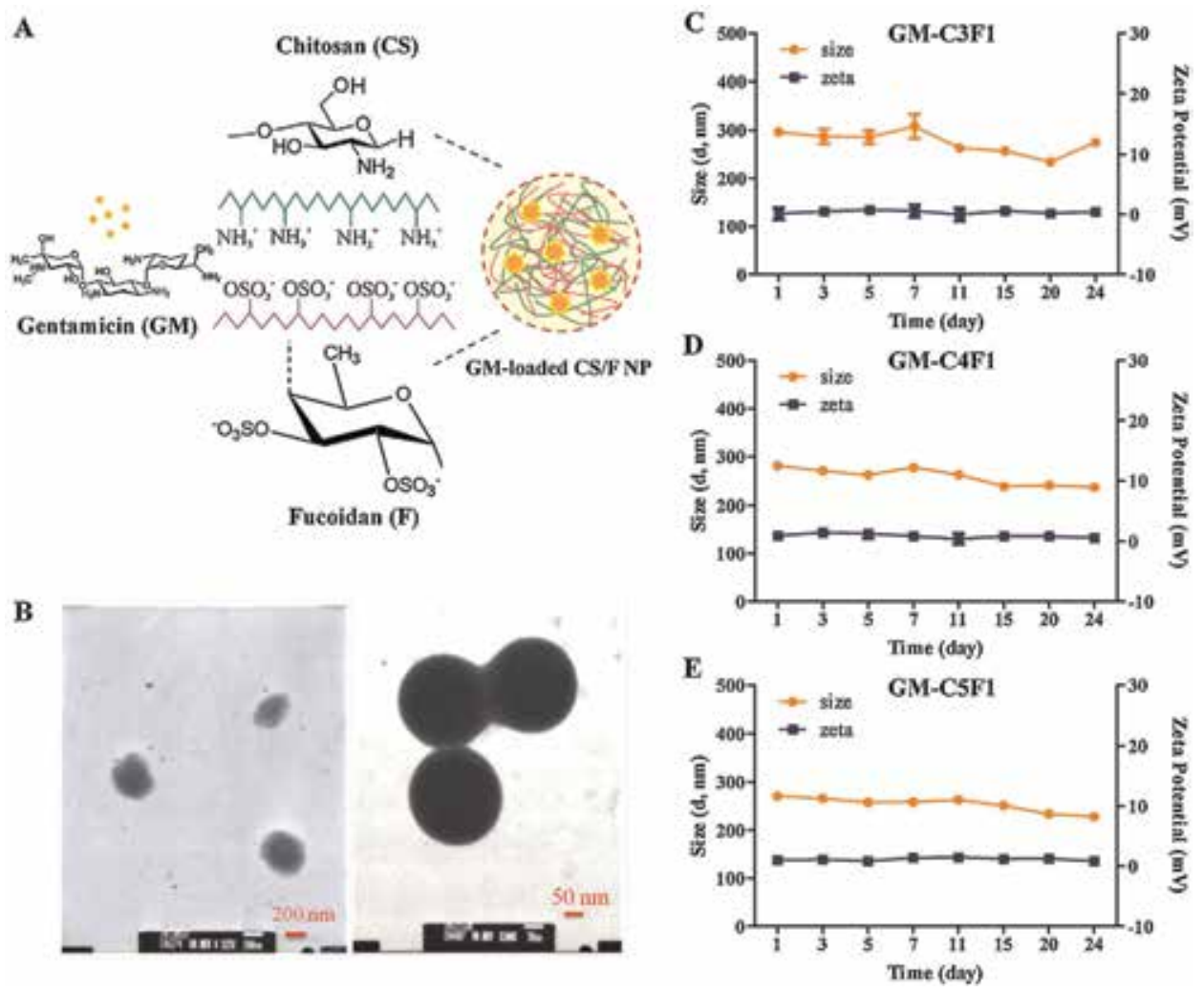

Figure 2.

(A) The preparation of the GM-loaded CS/F NPs. (B) TEM of the GM-C5F1 NPs. (C-E) Long-term stability of the series of CS/F NPs. 
Abdallah Makhlof et al. synthesized a thiomer derivative of glycol CS (GCS), which was coupled with thioglycolic acid (TGA) [65]. The NPs were prepared with GCS and GCS-TGA by ionic gelation for the pulmonary delivery of peptides. The NPs were positively charged and had sizes in the range of $230-330 \mathrm{~nm}$. They also showed high calcitonin entrapment. After intratracheal administration, the mucoadhesion capacity of the GCS-TGA NPs was much better than that of nonthiolated NPs in rats. The toxic effect of the NPs with lung tissue was confirmed with negligible epithelial damage and toxicity. The NPs could enhance the pulmonary absorption of the delivered peptides, and the calcitonin-loaded GCS and GCSTGA NPs showed efficient hypocalcemic effect. The GCS and its thiomer derivative could be used as potential PDDS for delivering peptides.

In another work, Adriana Trapani et al. had prepared CS and GCS NPs containing Lipoid S100 for the systemic delivery of low molecular weight heparin (LMWH) by pulmonary administration [66]. The NPs were prepared by ionotropic gelation method. The NPs were positively charged and with nanoscale size. Efficient drug entrapment and good mucoadhesive property could be achieved by using these NPs. The LMWH was effectively delivered into the lung by the aerosolization of the drug-loaded NPs. These results revealed the promising characteristics of the CS-based NPs, which were highly applicable for PDDS.

Tejal Rawal et al. designed a NP-based dry powder formulation of rifampicin (RFM) for achieving local and sustained targeting of anti-tubercular drugs to reduce dose and frequency [67]. The drug-loaded NPs were formulated by the ionic gelation probe sonication method. The optimized formulation had a small particle size of $124.1 \mathrm{~nm}$ with an entrapment efficiency of $72 \%$. No toxicity was found by in vitro and in vivo experiments. The pharmacokinetic assay verified that the NP formulation would serve a better alternative because it could minimize the frequent dosing schedule than conventional dry powder inhalation and market formulation. The RFM-loaded NPs open new avenues for developing therapeutic interventions for lung tuberculosis.

\subsubsection{CS-modified liposomes}

Liposomes are primarily used as PDDS for the treatment of respiratory distress syndrome and other lung diseases [68]. The drugs carried by liposomes mainly have stable physicochemical properties and strong lipophilicity. Liposome-based drug carrier has been one of the research hotspots in pharmaceutics [69, 70]. At present, drug-loaded liposome preparations, such as for amphotericin B, daunorubicin, doxorubicin, cytarabine, and morphine, have been successively developed [71]. The liposome preparations of these drugs have many unique advantages compared with their common preparations. Liposome-based pulmonary administration has the advantages of rapid absorption, little irritation, good tolerance, high safety, controllable drug release, and improved bioavailability [72]. The encapsulation of the drug in liposomes affects the pharmacokinetic property of the drug and prolongs drug half-life. Moreover, $85 \%$ of the components of the alveolar surface are phospholipids, which can facilitate the liposomes accumulate and enter the lung tissue. Liposome-loaded macromolecules with low lipophilicity can significantly improve bioavailability, and liposomes can also reduce the side effects of the toxic drugs to normal tissues by pulmonary administration.

Marco Zaru et al. prepared CS-coated liposomes and used them as drug carriers for pulmonary delivery by nebulization [73]. Rifampicin (RFM) was loaded in the CS-coated liposomes with different lipid compositions. By coating with CS, the encapsulation efficiency of the liposomes increased slightly, and after nebulization, the stability also remarkably increased. The mucoadhesive capacity of the 
CS-coated liposomes was much better than that of the noncoated ones. The cytotoxicity of the RFM-loaded CS-coated liposomes on A549 cells was much lower than that of the free drug. The results showed that the CS coating could significantly enhance the mucoadhesive capacity of the liposomes, and these CS-coated liposomes could be potential drug carriers for pulmonary administration by nebulization.

In another study, Mitsutaka Murata et al. investigated the surface modification of liposomes by CS for pulmonary delivery [74]. The surface of the liposomes was modified with CS oligosaccharide (oligoCS) and polyvinyl alcohol with a hydrophobic anchor (PVA-R). The association study showed that the PVA-R modification decreased the interaction between liposomes and A549 cells. By contrast, the oligoCS modification could significantly promote the cellular interaction. After pulmonary administration to rats, the peptide elcatonin (eCT) loaded oligoCS or PVA-R modified liposomes exhibited significantly enhanced therapeutic efficacy. The oligoCS-modified liposomes could adhere to the lung tissues and open the tight junctions between cells, which remarkably improved the drug absorption rate. On the other hand, the PVA-R-modified liposomes could induce a sustained absorption through the long-term eCT retention in lung fluid. The results verified that the surface-modification of liposomes with oligoCS and PVA-R could be used as effective PDDS for peptide pulmonary administration.

\subsubsection{CS-based active targeting DDS}

The pulmonary inhalation preparations are usually directly transported into the lung. Thus, they can passively target to the lung tissues by pulmonary administration. The deposition site of the drugs in the lung can be controlled by regulating the physicochemical and functional properties of the drug carriers to meet the disease treatment requirements. In addition to passive targeting, active targeting systems have more applications in precise and efficient disease treatment. Active targeting systems utilize ligands or antibody-modified carriers to deliver the drugs to the specifically targeted tissues or cells, even intracellular organelles, thereby improving drug efficacy and reducing toxicity and side effects $[75,76]$. The drug carriers could be chemically modified with active ligands, such as sugar moieties (galactose, mannose, and glucose) or specific ligands, such as folic acid (FA), transferrin (Tf), and Arg-Gly-Asp (RGD) peptide [77-79]. Antibody-mediated active targeting is also a primary strategy for delivering the drugs to the specific parts of the body [80].

$\mathrm{Hu}$-Lin Jiang et al. had prepared a folate-CS-graft-polyethylenimine (FC-g-PEI) copolymer for cancer cell-targeting gene delivery [81]. FC-g-PEI could effectively load and protect the shRNA. The copolymer also showed decreased cytotoxicity compared with the PEI control. Compared with the untargeted polymer, FC-g-PEI was a more efficient Akt1 shRNA carrier, and it exhibited effective cancer celltargeting ability. Moreover, aerosol delivery of the FC-g-PEI/Akt1 shRNA NPs effectively inhibited lung tumorigenesis in the urethane-induced lung cancer model mice via Akt signaling pathway. The results demonstrated that the FC-g-PEI could be applied for the shRNA gene therapy via aerosol delivery.

Yongfeng Luo et al. developed an inhalable $\beta 2$-adrenoceptor ligand-directed guanidinylated-CS (GCS) carrier for targeted lung delivery of siRNA [82]. GCS could effectively condense the siRNA and form complex NPs. Compared with pristine CS, the siRNA-loaded GCS NPs exhibited lower cytotoxicity and higher cellular internalization, which finally resulted in promoted gene silence efficiency. Salbutamol (a $\beta 2$-adrenoceptor agonist) was further chemically coupled to the GCS to enhance the targeting specificity of the siRNA-loaded NPs. The gene silence efficacy was remarkably increased both in vitro and in vivo. The carrier could 
protect the siRNA against the destructive shear forces generated by mesh-based nebulizers. Aerosol treatment also improved the size distribution of the NPs, which was beneficial in promoting the transfection efficiency. This CS-based targeting DDS had potential applications for siRNA delivery in lung disease treatment by aerosol inhalation.

Suhui Ni et al. had prepared $\gamma$-aminobutyric acid type $B\left(G_{A B A}\right)$ receptor ligand-directed NPs for survivin siRNA delivery [83]. The NPs were synthesized by baclofen (Bac)-functionalized trimethyl CS (Bac-TMC) with tripolyphosphate (TPP) as an ionic crosslinker. GABAB receptor agonist Bac was initially introduced into TMC as a novel ligand. The Bac-TMC/TPP NPs could promote the cellular uptake of siRNA by interacting with the $\mathrm{GABA}_{\mathrm{B}}$ receptor, which further induced effective gene silence and cell apoptosis. Mannitol microparticles were further utilized for the pulmonary delivery of the siRNA-loaded NPs via pressurized metered dose inhalers (pMDI). The obtained formulation had good aerodynamic properties, which benefited the deep lung deposition. The pMDI formulation containing Bac-TMC/TPP NPs could be a potential PDDS for siRNA.

Recently, Ting Wu et al. had developed a genipin-crosslinked carboxymethyl CS nanogel for lung-targeted delivery of isoniazid (INH) and rifampin (RMP) [84]. The dual drug-loaded nanogel particles (NGPs) had a uniform particle size from 60 to $130 \mathrm{~nm}$ with spherical morphology. The NGPs had sustained release behavior in simulated lung fluid. The dual drug-loaded NGPs had high antibacterial activity against multidrug-resistant Mycobacterium tuberculosis, and also could realize longterm antibacterial activity. Further in vivo evaluation exhibited that alveolar delivery of NGPs had satisfactory deposition of drug within the lung with lower toxicity. The results indicated that the NGPs would be a potential dosage form for treating against multidrug-resistant Mycobacterium tuberculosis.

\section{Future perspectives}

Pulmonary administration is a promising route for drug delivery to prevent and treat diseases, especially for delivering the drugs for lung diseases and some small molecule drugs with low absorption rate when in oral dosage form, also suitable for some traditional Chinese medicines and peptide or protein drugs. PDDS can effectively deliver the therapeutic drugs to the target sites, and improve the drug bioavailability and therapeutic efficacy. Inhalation is a noninvasive method for pulmonary administration, and the inhaled drugs can directly enter the blood circulation through the absorption of the alveolar epithelium. Pulmonary administration can enhance the drug absorption rate, reduce systemic side-effects, and improve the compliance of long-term medication. By transforming the drugs into dry powder inhalation formulations, drug degradation can be avoided. The therapeutic effect of the PDDS is mainly influenced by the physicochemical properties of the DDS, the dosage forms, and the administration devices, and also some other factors. The increase of the amount of drug delivered into the lung and the promotion of drug absorption rate are the key factors to improve the therapeutic efficiency of pulmonary administration. The application of sustained or controlled release preparations is an important method to prolong drug action time, enhance drug bioavailability, and improve patient compliance. In recent years, many controlled release preparations or active targeting preparations for pulmonary drug delivery have been constructed by drug-loaded microparticles, liposomes, and NPs. These multifunctional drug carriers have gained increasing popularity in pulmonary administration. It is really inspiring for us to see that some of the PDDS have already been applied to treat patients in clinic and become commercially available 
commodities with promising prospects. And in the coming years, great progress will be made in PDDS research by the development of drug delivery devices and pharmaceutical preparation technology.

The PDDS constructed with biomaterials has been a hot research direction in medicine and pharmacy fields for decades. Among these biomaterials, CS, which is the only natural cationic polysaccharide, has been recognized as one of the most versatile and promising functional biomaterials. Moreover, CS is also one of the most abundant regeneration resources, second only to the cellulose. After decades of research, CS has been recognized as a nontoxic, biocompatible (especially with respiratory cells) and biodegradable polymer. Moreover, CS can accommodate both hydrophilic and hydrophobic drugs due to its amphiphilic properties [5]. The excellent performances of CS as a building component of DDS have been confirmed by many studies, and it has been identified as a novel drug delivery carrier with broad application prospects, especially for sustained and controlled drug release. Due to the unique features, CS can assist the drugs accomplishing local and systemic delivery, realizing high mucoadhesion, and efficient drug absorption in target tissues, which is especially applicative for PDDS. However, CS is insoluble in aqueous solutions at neutral and alkaline $\mathrm{pH}$, and it only can be dissolved in aqueous acidic media $(\mathrm{pH}$ $<5$ ). This frustrating issue has badly limited CS's application range, and there is an urgent need for developing convenient and practical modification strategies to improve its solubility. Up to now, CS has been approved as safe by US-FDA and EU for dietary use and wound dressing applications [85]. And additionally, CS has only been approved by the European Pharmacopeia as a pharmaceutical excipient for oral preparations. The safety issues of CS and its derivatives in pulmonary delivery and other administration routes have not been fully understood and still remain to be further evaluated. Therefore, the safety issue is a noteworthy research gap remained to be filled in the future work, for figuring out the potential adverse effects of CS and its derivatives in humans. At the same time, appropriate engineering of designing and modifying CS and its derivatives is highly demanded to optimize the performance of the CS-based drug carriers, for meeting the special requirements of in vivo pulmonary drug delivery. We hope that in the near future, more advanced synthesis and modification methods will be developed. And in the meanwhile, the physicochemical property, toxicity, biodistribution, biocompatibility, and biodegradability of CS and its derivatives should also be thoroughly investigated in detail.

\section{Conclusions}

Despite all this, CS-based PDDS has already achieved considerable success in the past decades. It is believed that in the near future, with the rapid development of material science, biotechnology, genetic engineering, medical technology, and other scientific fields, people will have a more extensive and in-depth understanding of the unique properties of CS and its derivatives. We hope that more efficient CSbased PDDS will be designed, by developing novel material preparation strategies, advanced formulation methods, and improved inhalation technology. We believe that CS-based PDDS will play more important roles in the applications of local and systemic disease treatment in the near future. 
Applications of Chitosan in Pulmonary Drug Delivery

DOI: http://dx.doi.org/10.5772/intechopen.87932

\section{Acknowledgements}

The authors are thankful to the generous financial support from the National Natural Science Foundation of China (81774125) and National Key Technology R\&D Program of the Ministry of Science and Technology (2013GA740103).

\section{Conflict of interest}

The authors declare no conflicts of interest in this work.

\section{Author details}

Xiuwen Guan $^{1,2}$ and Weifen Zhang ${ }^{1,2 *}$

1 College of Pharmacy, Weifang Medical University, Weifang, Shandong, PR China

2 Shandong Engineering Research Center for Smart Materials and Regenerative Medicine, Weifang, Shandong, PR China

*Address all correspondence to: zhangwf@wfmc.edu.cn

\section{IntechOpen}

(C) 2019 The Author(s). Licensee IntechOpen. This chapter is distributed under the terms of the Creative Commons Attribution License (http://creativecommons.org/licenses/ by/3.0), which permits unrestricted use, distribution, and reproduction in any medium, provided the original work is properly cited. (cc) BY 


\section{References}

[1] Patton JS, Byron PR. Inhaling medicines: Delivering drugs to the body through the lungs. Nature Reviews Drug Discovery. 2007;6(1):67-74. DOI: $10.1038 / \mathrm{nrd} 2153$

[2] Patil J, Sarasija S. Pulmonary drug delivery strategies: A concise, systematic review. Lung India: Official Organ of Indian Chest Society. 2012; 29(1):44-49. DOI: $10.4103 /$ 0970-2113.92361

[3] Mansour HM, Rhee Y-S, Wu X. Nanomedicine in pulmonary delivery. International Journal of Nanomedicine. 2009;4:299-319. DOI: 10.2147/IJN.S4937

[4] Agoram B, Woltosz WS, Bolger MB. Predicting the impact of physiological and biochemical processes on oral drug bioavailability. Advanced Drug Delivery Reviews. 2001;50:S41S67. DOI: 10.1016/S0169-409X(01) 00179-X

[5] Islam N, Ferro V. Recent advances in chitosan-based nanoparticulate pulmonary drug delivery. Nanoscale. 2016;8(30):14341-14358. DOI: 10.1039/ C6NR03256G

[6] Agu RU, Ugwoke MI, Armand M, Kinget R, Verbeke N. The lung as a route for systemic delivery of therapeutic proteins and peptides. Respiratory Research. 2001;2(4): 198-209. DOI: $10.1186 / \mathrm{rr} 58$

[7] Qaseem A, Wilt TJ, Weinberger SE, Hanania NA, Criner G, van der Molen $\mathrm{T}$, et al. Diagnosis and management of stable chronic obstructive pulmonary disease: A clinical practice guideline update from the American College of Physicians, American College of Chest Physicians, American Thoracic Society, and European Respiratory Society. Annals of Internal Medicine. 2011; 155(3):179-191. DOI: $10.7326 /$ 0003-4819-155-3-201108020-00008
[8] Hoppentocht M, Hagedoorn P, Frijlink H, De Boer A. Technological and practical challenges of dry powder inhalers and formulations. Advanced Drug Delivery Reviews. 2014;75:18-31. DOI: 10.1016/j.addr.2014.04.004

[9] Pham D-D, Fattal E, Tsapis N. Pulmonary drug delivery systems for tuberculosis treatment. International Journal of Pharmaceutics. 2015;478(2): 517-529. DOI: 10.1016/j.ijpharm.2014. 12.009

[10] Liang Z, Ni R, Zhou J, Mao S. Recent advances in controlled pulmonary drug delivery. Drug Discovery Today. 2015; 20(3):380-389. DOI: 10.1016/j.

drudis.2014.09.020

[11] Manivasagan P, Oh J. Marine polysaccharide-based nanomaterials as a novel source of nanobiotechnological applications. International Journal of Biological Macromolecules. 2016;82: 315-327. DOI: 10.1016/j.ijbiomac.2015. 10.081

[12] Sonia T, Sharma CP. Chitosan and its derivatives for drug delivery perspective. In: Chitosan for Biomaterials I. Berlin, Germany: Springer; 2011. pp. 23-53. DOI: $10.1007 /$ 12_2011_117

[13] Singh Dhillon G, Kaur S, Jyoti Sarma S, Kaur Brar S, Verma M, Yadagiri Surampalli R. Recent development in applications of important biopolymer chitosan in biomedicine, pharmaceuticals and personal care products. Current Tissue Engineering. 2013;2(1):20-40. DOI: 10.2174/ 2211542011302010004

[14] Yamamoto H, Kuno Y, Sugimoto S, Takeuchi H, Kawashima Y. Surfacemodified PLGA nanosphere with chitosan improved pulmonary delivery of calcitonin by mucoadhesion and opening of the intercellular tight junctions. Journal of Controlled Release. 
2005;102(2):373-381. DOI: 10.1016/j. jconrel.2004.10.010

[15] Fröhlich E, Mercuri A, Wu S, Salar-Behzadi S. Measurements of deposition, lung surface area and lung fluid for simulation of inhaled compounds. Frontiers in Pharmacology. 2016;7:181. DOI: 10.3389/

fphar.2016.00181

[16] Patton JS, Fishburn CS, Weers JG. The lungs as a portal of entry for systemic drug delivery. Proceedings of the American Thoracic Society. 2004; 1(4):338-344. DOI: 10.1513/ pats.200409-049TA

[17] Patton JS. Mechanisms of macromolecule absorption by the lungs. Advanced Drug Delivery Reviews. 1996; 19(1):3-36. DOI: 10.1016/0169-409X (95) 00113-L

[18] Weibel ER. Morphological basis of alveolar-capillary gas exchange. Physiological Reviews. 1973;53(2): 419-495. DOI: 10.1152/physrev.1973. 53.2.419

[19] Stone KC, Mercer RR, Gehr P, Stockstill B, Crapo JD. Allometric relationships of cell numbers and size in the mammalian lung. American Journal of Respiratory Cell and Molecular Biology. 1992;6(2):235-243. DOI: 10.1165/ajrcmb/6.2.235

[20] Smola M, Vandamme T, Sokolowski A. Nanocarriers as pulmonary drug delivery systems to treat and to diagnose respiratory and non respiratory diseases. International Journal of Nanomedicine. 2008;3(1):1-19. DOI: 10.1016/ S0378-7753(01)01033-3

[21] Ricciardolo FL, Blasi F, Centanni S, Rogliani P. Therapeutic novelties of inhaled corticosteroids and bronchodilators in asthma. Pulmonary Pharmacology \& Therapeutics. 2015;33:1-10. DOI: 10.1016/j.pupt.2015.05.006
[22] Chono S, Fukuchi R, Seki T, Morimoto K. Aerosolized liposomes with dipalmitoyl phosphatidylcholine enhance pulmonary insulin delivery. Journal of Controlled Release. 2009; 137(2):104-109. DOI: 10.1016/j. jconrel.2009.03.019

[23] Kwon MJ, Bae JH, Kim JJ, Na K, Lee ES. Long acting porous microparticle for pulmonary protein delivery. International Journal of Pharmaceutics. 2007;333(1-2):5-9. DOI: 10.1016/j. ijpharm.2007.01.016

[24] Chow AH, Tong HH, Chattopadhyay P, Shekunov BY. Particle engineering for pulmonary drug delivery. Pharmaceutical Research. 2007;24(3):411-437. DOI: 10.1007/ s11095-006-9174-3

[25] Frampton MW, Stewart JC, Oberdörster G, Morrow PE, Chalupa D, Pietropaoli AP, et al. Inhalation of ultrafine particles alters blood leukocyte expression of adhesion molecules in humans. Environmental Health Perspectives. 2005;114(1):51-58. DOI: 10.1289/ehp.7962

[26] Todoroff J, Vanbever R. Fate of nanomedicines in the lungs. Current Opinion in Colloid \& Interface Science. 2011;16(3):246-254. DOI: 10.1016/j. cocis.2011.03.001

[27] Scheuch G, Kohlhaeufl MJ, Brand P, Siekmeier R. Clinical perspectives on pulmonary systemic and macromolecular delivery. Advanced Drug Delivery Reviews. 2006;58(9-10):996-1008. DOI: 10.1016/j.addr.2006.07.009

[28] Zhang J, Wu L, Chan H-K, Watanabe W. Formation, characterization, and fate of inhaled drug nanoparticles. Advanced Drug Delivery Reviews. 2011;63(6):441-455. DOI: 10.1016/j.addr.2010.11.002

[29] Kumar MNR. A review of chitin and chitosan applications. Reactive and 
Functional Polymers. 2000;46(1):1-27. DOI: $10.1016 / S 1381-5148(00) 00038-9$

[30] Dutta PK, Dutta J, Tripathi V. Chitin and chitosan: Chemistry, properties and applications. Journal of Scientific \& Industrial Research. 2004; 63:20-31. DOI: 10.1016/j.foodhyd.2010. 08.008

[31] Yuan Q, Shah J, Hein S, Misra R. Controlled and extended drug release behavior of chitosan-based nanoparticle carrier. Acta Biomaterialia. 2010;6(3): 1140-1148. DOI: 10.1016/j.actbio. 2009.08.027

[32] Wang JJ, Zeng ZW, Xiao RZ, Xie T, Zhou GL, Zhan XR, et al. Recent advances of chitosan nanoparticles as drug carriers. International Journal of Nanomedicine. 2011;6:765-774. DOI: 10.2147/IJN.S17296

[33] Boonsongrit Y, Mitrevej A, Mueller BW. Chitosan drug binding by ionic interaction. European Journal of Pharmaceutics and Biopharmaceutics. 2006;62(3):267-274. DOI: 10.1016/j. ejpb.2005.09.002

[34] Shukla SK, Mishra AK, Arotiba OA, Mamba BB. Chitosan-based nanomaterials: A state-of-the-art review. International Journal of Biological Macromolecules. 2013;59: 46-58. DOI: 10.1016/j.ijbiomac. 2013.04.043

[35] Gorzelanny C, Pöppelmann B, Pappelbaum K, Moerschbacher BM, Schneider SW. Human macrophage activation triggered by chitotriosidasemediated chitin and chitosan degradation. Biomaterials. 2010;31(33): 8556-8563. DOI: $10.1016 /$ j.biomaterials. 2010.07.100

[36] Fei Liu X, Lin Guan Y, Zhi Yang D, Li Z, De Yao K. Antibacterial action of chitosan and carboxymethylated chitosan. Journal of Applied Polymer Science. 2001;79(7):1324-1335. DOI:
10.1002/1097-4628(20010214)79:7< 1324::AID-APP210>3.0.CO;2-L

[37] Chung Y-C, Chen C-Y. Antibacterial characteristics and activity of acidsoluble chitosan. Bioresource Technology. 2008;99(8):2806-2814. DOI: 10.1016/j.biortech.2007.06.044

[38] Bansal V, Sharma PK, Sharma N, Pal OP, Malviya R. Applications of chitosan and chitosan derivatives in drug delivery. Advances in Biological Research. 2011;5(1):28-37

[39] Yeh T-H, Hsu L-W, Tseng MT, Lee P-L, Sonjae K, Ho Y-C, et al. Mechanism and consequence of chitosan-mediated reversible epithelial tight junction opening. Biomaterials. 2011;32(26): 6164-6173. DOI: 10.1016/j.biomaterials. 2011.03.056

[40] Illum L, Jabbal-Gill I, Hinchcliffe M, Fisher A, Davis S. Chitosan as a novel nasal delivery system for vaccines. Advanced Drug Delivery Reviews. 2001;51(1-3):81-96. DOI: 10.1016/ S0169-409X(01)00171-5

[41] Agnihotri SA, Mallikarjuna NN, Aminabhavi TM. Recent advances on chitosan-based micro-and nanoparticles in drug delivery. Journal of Controlled Release. 2004;100(1):5-28. DOI: 10.1016/j.jconrel.2004.08.010

[42] Cook RO, Pannu RK, Kellaway IW. Novel sustained release microspheres for pulmonary drug delivery. Journal of Controlled Release. 2005;104(1):79-90. DOI: 10.1016/j.jconrel.2005.01.003

[43] Sakagami M, Byron PR. Respirable microspheres for inhalation. Clinical Pharmacokinetics. 2005;44(3):263-277. DOI: $10.2165 / 00003088-200544030$ 00004

[44] Edlund U, Albertsson A-C. Degradable polymer microspheres for controlled drug delivery. In: Degradable Aliphatic Polyesters. Berlin, Germany: 
Springer; 2002. pp. 67-112. DOI: 10.1007/3-540-45734-8_3

[45] Mathiowitz E, Jacob JS, Jong YS, Carino GP, Chickering DE, Chaturvedi $\mathrm{P}$, et al. Biologically erodable microspheres as potential oral drug delivery systems. Nature. 1997; 386(6623):410-414. DOI: 10.1038/ $386410 \mathrm{a} 0$

[46] Liping W, Weihua W, Qian L, Qian Z, Yong W, Wei L, et al. The preparation of functionalized crosslinked macroporous chitosan microspheres and their adsorption properties for bilirubin. In: Macromolecular Symposia. Wiley Online Library; 2010. pp. 179-187. DOI: 10.1002/masy.200900070

[47] Kumar V, Lewis SA, Mutalik S, Shenoy DB, Udupa N. Biodegradable microspheres of curcumin for treatment of inflammation. Indian Journal of Physiology and Pharmacology. 2002; 46(2):209-217

[48] Mi F-L, Shyu S-S, Chen C-T, Lai J$\mathrm{Y}$. Adsorption of indomethacin onto chemically modified chitosan beads. Polymer. 2002;43(3):757-765. DOI: 10.1016/S0032-3861(01)00580-8

[49] He P, Davis SS, Illum L. Chitosan microspheres prepared by spray drying. International Journal of Pharmaceutics. 1999;187(1):53-65. DOI: 10.1016/ S0378-5173(99)00125-8

[50] Thanoo BC, Sunny M, Jayakrishnan A. Cross-linked chitosan microspheres: Preparation and evaluation as a matrix for the controlled release of pharmaceuticals. The Journal of Pharmacy and Pharmacology. 1992; 44(4):283-286. DOI: $10.1111 /$ j.2042-7158.1992.tb03607.x

[51] Zhang WF, Chen XG, Li PW, He QZ, Zhou HY. Preparation and characterization of theophylline loaded chitosan/ $\beta$-cyclodextrin microspheres.
Journal of Materials Science: Materials in Medicine. 2008;19(1):305-310. DOI: 10.1007/s10856-006-0021-1

[52] Zhang WF, Chen XG, Li PW, Liu CS, He QZ. Preparation and characterization of carboxymethyl chitosan and $\beta$-cyclodextrin microspheres by spray drying. Drying Technology. 2007;26(1):108-115. DOI: $10.1080 / 07373930701781736$

[53] Zhang W, Chen X, Li P, He Q, Zhou $\mathrm{H}$, Cha D. Chitosan and $\beta$-cyclodextrin microspheres as pulmonary sustained delivery systems. Journal of Wuhan University of Technology-Materials Science Edition. 2008;23(4):541-546. DOI: $10.1007 / \mathrm{s} 11595-006-4541-9$

[54] Zhang WF, Zhou HY, Chen XG, Tang SH, Zhang JJ. Biocompatibility study of theophylline/chitosan/ $\beta$ cyclodextrin microspheres as pulmonary delivery carriers. Journal of Materials Science: Materials in Medicine. 2009;20(6):1321-1330. DOI: 10.1007/s10856-008-3680-2

[55] Zhou H-Y, Zhou D-J, Zhang W-F, Jiang L-J, Li J-B, Chen X-G.

Biocompatibility and characteristics of chitosan/cellulose acetate microspheres for drug delivery. Frontiers of Materials Science. 2011;5(4):367-378. DOI:

10.1007/s11706-011-0146-0

[56] Liu K, Chen W, Yang T, Wen B, Ding $\mathrm{D}$, Keidar M, et al. Paclitaxel and quercetin nanoparticles co-loaded in microspheres to prolong retention time for pulmonary drug delivery. International Journal of Nanomedicine. 2017;12:8239-8255. DOI: 10.2147/IJN.S147028

[57] Cunha L, Rodrigues S, Rosa AM, Faleiro L, Buttini F, Grenha A. Inhalable chitosan microparticles for simultaneous delivery of isoniazid and rifabutin in lung tuberculosis treatment. Drug Development and Industrial Pharmacy. 2019;45(8):1313-1320. DOI: $10.1080 / 03639045.2019 .1608231$ 
[58] Cho K, Wang X, Nie S, Shin DM. Therapeutic nanoparticles for drug delivery in cancer. Clinical Cancer Research. 2008;14(5):1310-1316. DOI: 10.1158/1078-0432.CCR-07-1441

[59] Kumari A, Yadav SK, Yadav SC. Biodegradable polymeric nanoparticles based drug delivery systems. Colloids and Surfaces B, Biointerfaces. 2010; 75(1):1-18. DOI: 10.1016/j.colsurfb. 2009.09.001

[60] Wilczewska AZ, Niemirowicz K, Markiewicz KH, Car H. Nanoparticles as drug delivery systems. Pharmacological Reports. 2012;64(5):1020-1037. DOI: 10.1016/S1734-1140(12)70901-5

[61] Rytting E, Nguyen J, Wang X, Kissel T. Biodegradable polymeric nanocarriers for pulmonary drug delivery. Expert Opinion on Drug Delivery. 2008;5(6):629-639. DOI: 10.1517/17425247.5.6.629

[62] Bailey MM, Berkland CJ. Nanoparticle formulations in pulmonary drug delivery. Medicinal Research Reviews. 2009;29(1):196-212. DOI: $10.1002 /$ med.20140

[63] Howard KA, Rahbek UL, Liu X, Damgaard CK, Glud SZ, Andersen MØ, et al. RNA interference in vitro and in vivo using a novel chitosan/siRNA nanoparticle system. Molecular Therapy. 2006;14(4):476-484. DOI: 10.1016/j.ymthe.2006.04.010

[64] Huang Y-C, Li R-Y, Chen J-Y, Chen $\mathrm{J}-\mathrm{K}$. Biphasic release of gentamicin from chitosan/fucoidan nanoparticles for pulmonary delivery. Carbohydrate Polymers. 2016;138:114-122. DOI: 10.1016/j.carbpol.2015.11.072

[65] Makhlof A, Werle M, Tozuka Y, Takeuchi H. Nanoparticles of glycol chitosan and its thiolated derivative significantly improved the pulmonary delivery of calcitonin. International Journal of Pharmaceutics. 2010;397
(1-2):92-95. DOI: 10.1016/j. ijpharm.2010.07.001

[66] Trapani A, Di Gioia S, Ditaranto N, Cioffi N, Goycoolea FM, Carbone A, et al. Systemic heparin delivery by the pulmonary route using chitosan and glycol chitosan nanoparticles. International Journal of Pharmaceutics. 2013;447(1-2):115-123. DOI: 10.1016/j. ijpharm.2013.02.035

[67] Rawal T, Parmar R, Tyagi RK, Butani S. Rifampicin loaded chitosan nanoparticle dry powder presents an improved therapeutic approach for alveolar tuberculosis. Colloids and Surfaces B, Biointerfaces. 2017;154: 321-330. DOI: 10.1016/j.colsurfb.

2017.03.044

[68] Schreier H, Gonzalez-Rothi RJ, Stecenko AA. Pulmonary delivery of liposomes. Journal of Controlled Release. 1993;24(1-3):209-223. DOI: 10.1016/0168-3659(93)90180-D

[69] Sercombe L, Veerati T, Moheimani F, Wu SY, Sood AK, Hua S. Advances and challenges of liposome assisted drug delivery. Frontiers in Pharmacology. 2015;6:286. DOI: 10.3389/fphar.2015. 00286

[70] Lian T, Ho RJ. Trends and developments in liposome drug delivery systems. Journal of Pharmaceutical Sciences. 2001;90(6):667-680. DOI: 10.1002/jps.1023

[71] Daraee H, Etemadi A, Kouhi M, Alimirzalu S, Akbarzadeh A. Application of liposomes in medicine and drug delivery. Artificial Cells, Blood Substitutes, and Biotechnology. 2016; 44(1):381-391. DOI: $10.3109 /$ 21691401.2014.953633

[72] Willis L, Hayes D, Mansour HM. Therapeutic liposomal dry powder inhalation aerosols for targeted lung delivery. Lung. 2012;190(3):251-262. DOI: $10.1007 / \mathrm{s} 00408-011-9360-\mathrm{x}$ 
[73] Zaru M, Manca M-L, Fadda AM, Antimisiaris SG. Chitosan-coated liposomes for delivery to lungs by nebulisation. Colloids and Surfaces B, Biointerfaces. 2009;71(1):88-95. DOI: 10.1016/j.colsurfb.2009.01.010

[74] Murata M, Nakano K, Tahara K, Tozuka Y, Takeuchi H. Pulmonary delivery of elcatonin using surfacemodified liposomes to improve systemic absorption: Polyvinyl alcohol with a hydrophobic anchor and chitosan oligosaccharide as effective surface modifiers. European Journal of Pharmaceutics and Biopharmaceutics. 2012;80(2):340-346. DOI: 10.1016/j. ejpb.2011.10.011

[75] Allen TM. Ligand-targeted therapeutics in anticancer therapy. Nature Reviews Cancer. 2002;2(10): 750-763. DOI: $10.1038 /$ nrc903

[76] Brannon-Peppas L, Blanchette JO. Nanoparticle and targeted systems for cancer therapy. Advanced Drug Delivery Reviews. 2012;64: 206-212. DOI: 10.1016/j.addr.2012. 09.033

[77] Byrne JD, Betancourt T, BrannonPeppas L. Active targeting schemes for nanoparticle systems in cancer therapeutics. Advanced Drug Delivery Reviews. 2008;60(15):1615-1626. DOI: 10.1016/j.addr.2008.08.005

[78] Vyas SP, Singh A, Sihorkar V. Ligand-receptor-mediated drug delivery: An emerging paradigm in cellular drug targeting. Critical Reviews in Therapeutic Drug Carrier Systems. 2001;18(1):1-76. DOI: 10.1615/CritRevTherDrugCarrierSyst. v18.11.10

[79] Zwicke GL, Ali Mansoori G, Jeffery CJ. Utilizing the folate receptor for active targeting of cancer nanotherapeutics. Nanotechnology Reviews. 2012;3(1):18496. DOI: 10.3402/nano.v3i0.18496
[80] Ansell SM, Harasym TO, Tardi PG, Buchkowsky SS, Bally MB, Cullis PR. Antibody conjugation methods for active targeting of liposomes. In: Drug Targeting. New Jersey, USA: Humana Press; 2000. pp. 51-68. DOI: 10.1385/ 1-59259-075-6:51

[81] Jiang H-L, Xu C-X, Kim Y-K, Arote $\mathrm{R}$, Jere $\mathrm{D}$, Lim $\mathrm{H}-\mathrm{T}$, et al. The suppression of lung tumorigenesis by aerosol-delivered folate-chitosan-graftpolyethylenimine/Akt1 shRNA complexes through the Akt signaling pathway. Biomaterials. 2009;30(29): 5844-5852. DOI: 10.1016/j.biomaterials. 2009.07.017

[82] Luo Y, Zhai X, Ma C, Sun P, Fu Z, Liu $W$, et al. An inhalable $\beta 2-$ adrenoceptor ligand-directed guanidinylated chitosan carrier for targeted delivery of siRNA to lung. Journal of Controlled Release. 2012; 162(1):28-36. DOI: 10.1016/j. jconrel.2012.06.005

[83] Ni S, Liu Y, Tang Y, Chen J, Li S, Pu $\mathrm{J}$, et al. GABAB receptor ligand-directed trimethyl chitosan/tripolyphosphate nanoparticles and their pMDI formulation for survivin siRNA pulmonary delivery. Carbohydrate Polymers. 2018;179:135-144. DOI: 10.1016/j.carbpol.2017.09.075

[84] Wu T, Liao W, Wang W, Zhou J, Tan W, Xiang W, et al. Genipincrosslinked carboxymethyl chitosan nanogel for lung-targeted delivery of isoniazid and rifampin. Carbohydrate Polymers. 2018;197:403-413. DOI: 10.1016/j.carbpol.2018.06.034

[85] Mohammed M, Syeda J, Wasan K, Wasan E. An overview of chitosan nanoparticles and its application in non-parenteral drug delivery. Pharmaceutics. 2017;9:53. DOI: 10.3390/pharmaceutics 9040053 


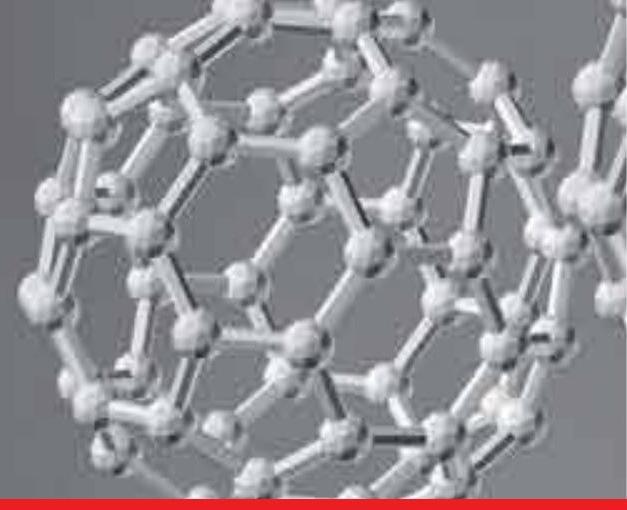

\section{Edited by Rajeev K. Tyagi, Neeraj Garg, Rahul Shukla and Prakash Singh Bisen}

Medical Nanotechnology and Nanomedicine introduces non-experts to the world of nanomedicine and its evolving organizational infrastructure. Considering the fluid nature of nano breakthroughs and the delicate balance between benefits and consequences as they apply to medicine, readers at all levels will gain a practical, understandable base of information on these developments so that they may take the greatest advantage of them. This practical reference investigates the impact of nanotechnology on applications in medicine and biomedical sciences, and the broader societal and economic effects. Eschewing technological details, it focuses on enhancing awareness of the business, regulatory, and administrative aspects of medical applications. It gives readers a critical, balanced, and realistic evaluation of existing nanomedicine developments and future prospects and provides an ideal foundation upon which to plan and make decisions.

Published in London, UK 